\title{
Claudio De Boni
}

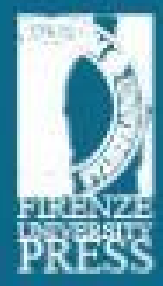

\section{Descrivere}

il futuro 
Università degli Studi di Firenze

Facoltà di Scienze Politiche «Cesare Alfieri»

Dipartimento di Studi sullo Stato

Seminario di Storia delle Idee Politiche

\section{Claudio De Boni}

\section{Descrivere il futuro}

Scienza e utopia in Francia nell' età del positivismo

Firenze University Press 
Descrizioni del futuro : scienza e utopia in Francia nell'età del positivismo / Claudio De Boni. - Firenze : Firenze University Press, 2003.

(Seminario di storia delle idee politiche. Nuova serie / Università degli studi di Firenze, Dipartimento di studi sullo Stato).

http://digital.casalini.it/fulltext/is.asp?isbn=8884530989

Stampa a richiesta disponibile su http://epress.unifi.it

ISBN 88-8453-098-9 (online)

ISBN 88-8453-099-7 (print)

146.4 (ed. 20)

Positivismo - Francia

Seminario di storia delle idee politiche

Comitato scientifico: Antonio Zanfarino, Sergio Caruso, Vittore Collina, Claudio De Boni, Brunella Casalini

Nuova Serie

Volumi pubblicati nella serie precedente:

S. Caruso, V. Collina, C. De Boni, Numeri e politica. Matematica risorse e progresso in Hume, Condorcet, Godwin

S. Caruso, Intellettuali e mondi possibili. Itinerari e problemi del pensiero politico moderno e contemporaneo

S. Caruso, Una certa idea dell'America. Il giovane Walzer e la New Left

() 2003 Firenze University Press

Università degli Studi di Firenze

Firenze University Press

Borgo Albizi, 28

50122 Firenze

http://epress.unifi.it

mail: e-press@unifi.it 


\section{Indice}

\section{L'ambigua eredità del comtismo}

1.1. I due tempi del pensiero di Comte 5

$\begin{array}{lr}\text { 1.2. Il positivismo da filosofia scientifica a progetto etico } & 10\end{array}$

1.3. Verso un'utopia conservatrice 18

1.4. La religione dell'Umanità e il suo culto 24

\section{Dopo Comte: positivismo moderato e positivismo radicale}

2.1. Littré e il positivismo della Terza Repubblica 35

2.2. La "settima scienza" del positivismo: Laffitte e la morale 41

2.3. Il ruolo sociale della scienza: Marcellin Berthelot 47

2.4. Un mondo governato dalla scienza: sogno o incubo? 55

\section{La "macchina del tempo"}

3.1. Renouvier e il fascino della "ucronia" 65

3.2. Il futuro come angoscia: a proposito di un'opera "dimenticata" di Jules Verne 74

3.3. Il nuovo mondo dopo la catastrofe: Tarde e la storia futura 82

3.4. Il ruolo ambiguo della scienza nell'ucronia di Halévy 91

\section{I conti con l'utopia}

4.1. Un modello di socialismo tecnologico: Zola e il riscatto dei lavoratori

4.2. Anatole France e l'utopia come possibilità 107

4.3. Il peso della storia 117

4.4. Un catalogo per il Novecento 123 



\section{L’ambigua eredità del comtismo}

\subsection{I due tempi del pensiero di Comte}

Nel 1863, commentando l'ampia serie di documenti sulla storia del positivismo da lui raccolta sotto il titolo Auguste Comte et la philosophie positive, Emile Littré elabora per primo un'immagine destinata a esercitare grande influenza sulla successiva percezione del ruolo di Comte nella cultura francese ed europea dell'Ottocento. L'immagine è quella della presunta esistenza di "due" Comte dai caratteri distinti. Il primo sarebbe quello degli anni trenta e dei primi anni quaranta, che nel Cours de philosophie positive è stato capace di costruire un sistema di pensiero innovativo, rispondente alla realtà oggettiva del mondo naturale e sociale. Il secondo è il Comte degli ultimi anni di vita, quelli in cui il fondatore del positivismo si sarebbe fatto prendere, secondo la valutazione di Littré, da una visione soggettiva del mondo non più ancorata alla realtà effettiva, ma frutto di immaginazione personale e di ansia per una nuova quanto confusa spiritualità. La distinzione di Littré fra "due" Comte, l'uno apprezzato e l'altro rifiutato, avrebbe costituito, lungo tutto il successivo scorcio di secolo, una linea critica consolidata soprattutto per i positivisti della Terza Repubblica. I quali si dimostreranno intenzionati ad assorbire del loro maestro solo il richiamo alla centralità della scienza nell'organizzazione del mondo, senza compromissioni di ordine strettamente religioso. Come è noto, il giudizio littréano da cui sto prendendo spunto segue di circa un decennio la rottura consumatasi nel 1852 all'interno della Société Positiviste fra Comte e Littré: tra il fondatore del positivismo, cioè, e colui che ne è stato uno dei più decisi estimatori e divulgatori, a metà degli anni quaranta, dalle colonne del giornale repubblicano "National". Questo fatto va tenuto presente perché potrebbe far pensare, con qualche fondamento, che l'opinione di Littré porti con sé qualche tratto di strumentalità o quantomeno di autogiustificazione: incolpare implicitamente della rottura un cambiamento sopravvenuto in Comte non solo solleva Littré dalle maggiori responsabilità in proposito, ma ha anche il pregio di non mettere in discussione la sua infatuazione precedente. Seguire la linea critica tracciata da Littré richiede insomma di 
procedere con cautela: eppure quel percorso va intrapreso, perché le distinzioni di Littré rimandano a valutazioni di squisito ordine politico. E solo portando al centro dell'attenzione il messaggio politico di Comte e i suoi intricati processi di formazione potremo valutare a pieno i caratteri dell'eredità, tutt'altro che lineare, da lui depositata per la cultura successiva.

Le ragioni della rottura fra Comte e Littré sono infatti varie e complesse ${ }^{1}$. Non mancano, come a suo tempo fra Comte e Saint-Simon, le incomprensioni personali e le gelosie intellettuali ${ }^{2}$ : ma a noi interessano soprattutto le questioni di ordine filosofico, culturale e politico, che si presentano in effetti corpose e decisive. La questione intellettuale di fondo è quella del rapporto tra la filosofia positiva e l'effettiva realtà storica. Ha in sé il positivismo la spiegazione e la soluzione di ogni problema evolutivo, o esso indica soltanto una serie di linee di tendenza, da arricchire indefinitamente con l'apporto della libera ricerca umana, come pensa Littré $^{3}$ ? Sul piano culturale, a tracciare una linea di demarcazione fra i due è il crescente spazio assunto dalle infatuazioni spiritualistiche nella pratica e nella predicazione dell'ultimo Comte, che il laico e austero Littré è ben lontano dal poter comprendere e accettare, se non nelle forme moderate del riconoscimento della necessità di credenze comuni, che ci si augura sempre meno in contrasto con l'evidenza scientifica. E non mancano infine, a determinare la rottura del 1852, che segue di così poco le esaltazioni e le disillusioni della rivoluzione del 1848, le ragioni di natura direttamente politica. Non mi riferisco soltanto ai richiami di ordine generale, che pure cominciano a segnare un distacco fra la critica radicale alla libertà individuale propugnata da Comte e le maggiori aperture dimostrate in proposito da Littré, ma anche alle più immediate scelte di schieramento. Mentre Comte inclina verso l'accettazione implicita del bonapartismo restaurato (o almeno verso il dialogo con il regime di Luigi Napoleone), Littré rimane saldamente ancorato a una concezione repubblicana che non accetta scorciatoie autoritarie sulla strada dell'auspicata instaurazione della società positiva. E tantomeno può valutare

\footnotetext{
${ }^{1}$ La ricostruzione più esauriente delle fasi e delle ragioni della rottura è quella proposta da $\mathrm{M}$. Larizza, Bandiera verde contro bandiera rossa. Auguste Comte e gli inizi della Société positiviste (1848-1852), Bologna, Il Mulino, 1999. Al documentatissimo libro della Larizza rinvio anche per la narrazione delle vicende riguardanti il positivismo nella sua stagione di più acuto avvicinamento alle problematiche politiche, quella appunto del 1848 e dintorni.

${ }^{2}$ Tra le ragioni di conflitto un peso rilevante, stando alle dichiarazioni offerte nel periodo della rottura da ambedue i contendenti, assume la separazione fra Comte e la moglie, Caroline Massin, avvenuta pochi anni prima. Littré attribuisce a questa separazione, e alla successiva esaltazione di Comte per Clotilde de Vaux, molti degli squilibri subiti da Comte e dalle sue idee a partire dalla metà degli anni quaranta. Nel frattempo egli continua a mantenere contatti amichevoli con la Massin: secondo Comte, ne diventa addirittura l'amante. Come che sia, lo stesso libro del 1863 curato da Littré vede la luce anche per la collaborazione della Massin nel fornirgli memorie e documenti sulle prime fasi della storia del positivismo. Su tutto ciò si vedano fra le altre le allusioni comprese in F.E. Manuel, Auguste Comte: l'incarnazione nel Grande Essere, in I profeti di Parigi, Bologna, Il Mulino, 1979.

${ }^{3}$ Sull'evoluzione del pensiero di Littré rinvio al mio La rivoluzione conservatrice. Emile Littré e il positivismo, Firenze, D’Anna, 1996.
} 
favorevolmente l'eventuale alleanza col bonapartismo, da sempre da lui giudicato "retrogrado", volto alla conservazione e non al progresso.

Di tutto questo, nel suo libro del 1863 Emile Littré ama far trasparire soprattutto i temi di ordine filosofico, facilmente traducibili, tuttavia, in argomenti di carattere politico. Il punto da cui parte la sua critica all'ultimo Comte si muove attorno al tema dell'unità, parola d'ordine sotto cui si cela l'aspirazione positivista a rappresentare il mondo come un tutto armonico. Esistono nel pensiero e nella realtà, spiega Littré con la consueta chiarezza, due tipi di unità, l'una soggettiva e l'altra oggettiva. "L'unità soggettiva è l'insieme delle condizioni mentali sotto cui noi conosciamo il vero, e l'insieme dei princìpi generali che ci guidano nelle applicazioni. L'unità oggettiva è l'insieme delle condizioni naturali sotto le quali il mondo sussiste, e l'insieme dei procedimenti applicati a ciascuna categoria di fenomeni" ${ }^{\prime 4}$. Rispettosi dichiarati della lezione di Hume, i positivisti accettano un processo di conoscenza basato sul dato empirico, certi però di potere prima o dopo attingere scientificamente a un'immagine unitaria della natura, in un'operazione agevolata dalla capacità umana di costruire con l'intelletto sistemi coordinati.

Quest'ultimo procedimento, continua Littré, non è un'invenzione del positivismo: anche la vecchia filosofia, mediante la metafisica, ha elaborato un'immagine unitaria del mondo. Quello che caratterizza i positivisti è però la capacità di ancorare l'unità soggettiva, la rappresentazione mentale, alla verità scientifica, senza più attardarsi nelle indimostrabili affermazioni della metafisica. "Metafisico" è, nel linguaggio positivista, tutto ciò che rimanda a una ragione astratta, mentre le costruzioni mentali auspicate dal movimento fondato da Comte dovrebbero trovare alimento nella concretezza. Sotto questo profilo il Littré del 1863 continua ad avere parole di elogio per il Comte del Cours de philosophie positive, nel quale il suo autore ha coniugato novità e grandezza: "novità, perché per la prima volta l'unità oggettiva appariva nel campo delle idee; grandezza, perché la concezione di Comte imprime per la prima volta all'unità soggettiva il sigillo di positività che le mancava"s. A Comte si deve insomma il modello più compiuto di unione filosofica fra le varie scienze speciali ${ }^{6}$ : anzi l'unico modello

\footnotetext{
${ }^{4}$ E. Littré, Auguste Comte et la philosophie positive, Paris, Hachette, 1864, pp. 183-184. Cito dalla seconda edizione del libro, uscita un anno dopo la prima.

${ }^{5}$ Ivi, p. 184.

${ }^{6}$ Ricordo che nel Cours Comte classifica le scienze speciali secondo una catena comprendente sei discipline: matematica, astronomia, fisica, chimica, biologia, sociologia. L'ordine stabilito dal fondatore del positivismo non è casuale, ma riflette i diversi tempi in cui, nella storia della conoscenza, le singole scienze hanno raggiunto lo stadio positivo: prima la matematica, ultima la sociologia. Ricordo ancora, anche se è fra gli aspetti più noti del pensiero di Comte, che il progresso della conoscenza umana attraversa per ogni scienza tre stadi. Il primo è quello teologico, nel quale l'origine dei fenomeni viene fatta risalire a cause divine; il secondo è quello metafisico, nel quale entrano in campo le idee astratte, pensate come le essenze delle cose. Nel terzo stadio, quello positivo, predomina finalmente il procedimento scientifico, interpretato da Comte come ricerca delle leggi generali che caratterizzano i fenomeni, eliminando dai processi di conoscenza aspetti indimostrabili come l'origine prima o i fini ultimi dei fenomeni stessi. L'ordine fra le scienze non è inoltre retto da un dato puramente storiografico, ma costituisce anche la griglia di una classificazione in base alla
} 
esistente, secondo l'entusiastica affermazione di Littré. E l'operazione si è potuta effettuare soprattutto perché l'autore del Cours ha saputo arricchire l'impianto delle scienze naturali con una nuova scienza del tutto "umana", la sociologia, le cui tracce principali si trovano calate nella storia ${ }^{7}$. Littré continua così ad accettare che faccia parte della filosofia positiva non solo un grande progetto di comprensione del mondo naturale nelle sue interne connessioni, ma anche un apparato di previsione e di valutazione in ambito sociale disponibile a proiettarsi in un disegno di governo della società nel suo progresso storico, comprensivo di istituzioni politiche e di mobilitazioni culturali e mentali.

Ma proprio nel momento in cui ribadisce l'importanza esercitata dalla sociologia come vertice dell'ordine gerarchico delle scienze, Littré comincia a prendere le distanze dalle posizioni che Comte (ora l'ultimo Comte, quello del Système de politique positive) ha voluto dedurre dall'impianto storico-sociale della filosofia positiva da lui stesso elaborato. Il primo Comte, secondo Littré, ha fatto uno sforzo titanico per mettere insieme senza contraddizioni unità soggettiva e unità oggettiva; il secondo, quello che si muove dalla metà degli anni quaranta alla morte, si è invece fatto riassorbire in una costruzione di pensiero soggettivistica (di cui la religione dell'Umanità, nei termini estesi delineati nel Système, è la creazione più emblematica), aggiungendo alle vecchie metafisiche e teologie una nuova costruzione individuale non suffragata dalla scienza. Nel suo libro del 1863 Littré offre molte spiegazioni di questa involuzione comtiana, anche di tipo biografico: ma quella più ricca di sviluppi critici è l'interpretazione dei cambiamenti comtiani riferita a pure questioni di pensiero. Secondo la lettura littréana Comte, dopo aver costruito un sistema unitario di comprensione della realtà sociale e storica dell'umanità, dopo aver in altri termini scoperto l'importanza della sociologia, non ha avuto la pazienza intellettuale di ricondurre le linee fondamentali della nuova scienza alla verifica dei fatti. Per quanto egli stesso abbia insegnato che "più una scienza è elevata gerarchicamente" (e la sociologia è la più elevata di tutte), "più la facoltà di trarre deduzioni ne risulta diminuita", la sua ansia di ricavare

complessità e alla concatenazione logica. Le scienze che per ultime raggiungono lo stadio positivo sono anche le più complesse, per la loro estensione progressiva al mondo vivente, e sono collegate dialetticamente a quelle che le hanno precedute. Se una scienza più elevata non può contraddire $\mathrm{i}$ princìpi di quelle che la precedono nella scala positiva della conoscenza, d'altra parte essa costituisce il loro sbocco necessario, in qualche modo il loro completamento. Da tutto ciò risulta una preminenza concettuale della sociologia come scopo del sapere positivo: una superiorità che, accennata nel Cours, viene ribadita ed enfatizzata nel Système de politique positive.

7 "Il sistema della filosofia positiva è, come ho già detto, una serie di filosofie delle scienze che si succedono in un ordine gerarchico. Se è molto difficile tracciare la filosofia di una scienza, fare quella di tutte le scienze è prodigioso: ma dopotutto, per cinque almeno i materiali esistevano già, e Comte ha avuto solamente il compito di trarne delle generalità luminose che ne catturassero e fissassero lo sviluppo, la correlazione, i princìpi. Niente di simile per la storia: lì occorreva nello stesso tempo creare una scienza che non esisteva e trarne a mano a mano la filosofia, compagna e corollario delle cinque filosofie che l'avevano preceduta. Lo scienziato e il filosofo lavoravano l'uno per l'altro; e Comte poté essere il primo dei filosofi della storia solo perché fu il primo degli scienziati in quest'ambito particolare" (E. Littré, Auguste Comte, cit., pp. 180-181).

${ }^{8}$ Ivi, p. 533. 
affermazioni politiche e religiose dall'interpretazione complessiva della storia costruita dal positivismo lo ha indotto a restaurare il metodo soggettivo, cioè a trasferire nella realtà senza verifiche le affermazioni di armonia poste a priori. "Da un punto di vista universale legittimamente conquistato", Comte "trae conseguenze non verificate dall'esperienza, ma fornite dalla sua immaginazione o, se si vuole, da una logica soggettiva",

Avanzando rilievi del genere Littré intende soprattutto porre al riparo parte dell'eredità positivista, quella laica e moderata che egli rappresenta, dagli influssi spiritualizzanti messi in atto dai discepoli più "ortodossi" di Comte: non per nulla tutto il libro del 1863 è pieno di denunce del fatto che il discorso del suo vecchio maestro è diventato sempre più religioso e sempre meno scientifico. Ma in gioco c'è una posta ancora più vasta della predicazione religiosa, una posta di cui lo stesso Littré diverrà cosciente solo in seguito: e cioè l'opportunità o meno di sciogliere le ambiguità di fondo del positivismo delle origini, oscillante tra ricerca e dogma, tra conoscenza e rifondazione del mondo, tra scienza e politica. Mentre Comte, come tenteremo di verificare fra poco, cerca di disinnescare le possibili contraddizioni proiettandosi su un terreno molto prossimo all'utopismo, Littré continua una sua personale revisione che, senza dirlo esplicitamente, comincia a porsi dubbi su uno dei capisaldi della filosofia comtiana.

Distinguere le possibilità deduttive della sociologia da quelle delle altre scienze equivale infatti a mettere in discussione la possibilità di usare uno strumento unitario di pensiero per penetrare da un lato nella natura dall'altro nella storia: equivale a rendere meno decisa la fiducia nel poter mantenere un'analogia di metodo fra le scienze della natura e la sociologia ${ }^{10}$. Così, mentre il positivismo diventa per l'ultimo Comte la spiegazione compiuta di ogni fenomeno naturale e umano, per l'ultimo Littré sarà soprattutto un metodo ancora in espansione, una filosofia in divenire che non possiede in sé tutta la verità, ma soltanto gli strumenti per avvicinarsi indefinitamente a essa. Allo stesso modo, la costruzione politica sottesa alla filosofia comtiana, cioè la società positiva, se per Comte, alla luce della

\footnotetext{
${ }^{9}$ Ibidem.

${ }^{10}$ Il metodo scientifico si basa, secondo Comte, sulla combinazione di tre elementi. Il primo è dato dall'osservazione, che deve imbrigliare l'immaginazione e ha quindi una portata eminentemente empirica, anche se non di un empirismo "cieco", che impedisca le generalizzazioni: l'osservazione deve quindi essere guidata da quello che Comte chiama lo "spirito d'insieme". Il secondo elemento è la sperimentazione, che consente di verificare l'esistenza di leggi naturali attraverso la ripetizione dei fenomeni a parità di condizioni. Il terzo è la comparazione tra i fenomeni che si verificano in realtà diverse. Comte è consapevole che i fenomeni storici e sociali hanno una consistenza diversa dai fenomeni naturali, e non possono in tutto e per tutto essere studiati come quest'ultimi: una differenza fondamentale è per esempio costituita dal fatto che è impossibile sperimentare in laboratorio i fenomeni sociali. Ma forme sperimentali analoghe a quelle naturali, o quantomeno studiabili con metodo scientifico, sono offerte dalla storia, specialmente nei periodi di perturbazione. Le epoche di crisi, esattamente come le malattie del corpo umano analizzate dalla medicina, possono rivelare molte cose sul funzionamento di un organismo sociale. Prende avvio da questa convinzione la grande attenzione rivolta nelle opere di Comte alla Rivoluzione francese, era patologica che segna però il punto di svolta da un'epoca all'altra: definitiva esplosione delle tesi distruttive della cultura metafisica, essa apre però nello stesso tempo la strada all'avvento della società positiva.
} 
filosofia della storia, può essere compiutamente espressa in tutte le sue articolazioni e comunicata come società ideale, per Littré è un obiettivo ancora lontano, e non così determinato come pensava il suo vecchio maestro.

\subsection{Il positivismo da filosofia scientifica a progetto etico}

A un primo avvicinamento, i caratteri di continuità fra il Cours de philosophie positive e il Système de philosophie positive sembrano per la verità maggiori di quanti Littré fosse disposto ad ammettere. Sia nell'una che nell'altra delle grandi sillogi del pensiero comtiano troviamo per esempio una delle costruzioni più tipiche del positivismo delle origini, la legge dei tre stadi come linea interpretativa della storia. E in ambedue le opere appaiono altri elementi basilari della politica positiva, come la delineazione di una società armonica fondata sulla collaborazione fra le classi, o la necessità della separazione fra il potere materiale e il potere spirituale. Quel che dà tuttavia ragione a Littré è la nuova gerarchia degli intendimenti e degli umori comtiani che contrassegna il Système rispetto al Cours: è, come vedremo meglio nella prosecuzione del nostro discorso, il progressivo abbandono dei toni descrittivi propri del ragionamento scientifico per assumere quelli ispirati propri del moralizzatore. Ad apertura della prefazione al primo tomo del Système, in posizione quindi strategica per la comunicazione delle intenzioni dell'autore, Comte afferma che è stato da sempre suo scopo quello di far convergere scienza e politica. Nel Cours egli ha preferito far prevalere in proposito l'esprit, la ragione, per "meglio caratterizzare la superiorità intellettuale del positivismo su ogni teologismo"". Ma nel Système è giunta l'ora di portare finalmente in superficie il coeur, il sentimento, perché solo facendo perno sulle pulsioni affettive è possibile aspirare alla realizzazione della società positiva, di un sistema cioè basato su un'estesa solidarietà fra disuguali.

Per un verso Comte sembra interpretare questa apertura al sentimento come aggiuntiva rispetto alla precedente propensione razionalistica. L'ordine sociale, si dice in più passaggi del Système, è il prodotto congiunto dell'intelligenza e della naturale, e perciò istintiva e affettiva, socialità degli uomini: per meglio praticare la seconda, occorre valorizzare anche la prima, che richiede capacità razionali e libertà di indagine. Ma più spesso si assiste, nella seconda grande opera di Comte, a un autentico rovesciamento di prospettiva fra i due termini rispetto al Cours. Se lì predominava la ragione, adesso la priorità va assicurata al sentimento, molto più efficace rispetto all'intelligenza nell' assicurare coesione sociale e rispondenza alle parole d'ordine del filosofo positivista, che ora si pone anzitutto come maestro di morale. Tanto che il libero uso della ragione applicata alla scienza viene avvertito dall'ultimo Comte come un problema insoluto all'interno del positivismo. Se da un lato il progresso della conoscenza richiede autonomia del sapere, dall'altro la

${ }^{11}$ A. Comte, Système de politique positive, ou Traité de sociologie instituant la religion de l'humanité, t. I, Paris, Larousse, 1890, p. 3. Cito da un'edizione uscita fra il 1890 e il '95, conforme a quella originale che aveva visto la luce fra il 1851 e il 1854 in quattro tomi (uno per anno). 
libertà di indagine può costituire una frattura individualistica nell'ordine comunitario desiderato. Fine della politica positiva (e fine della storia) sarà non per nulla quello di reprimere le tendenze egoistiche, anche sul piano della conoscenza, per farle consentire con la verità sociale. Ma proprio per ottenere questo risultato occorre frenare l'intelligenza col sentimento ${ }^{12}$.

Con queste osservazioni Comte aggiunge alla precedente fiducia sul metodo razionale un'avvertenza, forse condivisibile, sul fatto che la ragione umana, a volte portatrice di illuminazioni, altre volte di fuorvianti sottigliezze, può essere anche uno strumento di potere dell'uomo sull'uomo, non solo di liberazione collettiva. Ma il dubbio su una razionalità forse non sempre rispondente ai bisogni umani scivola verso una restaurazione spiritualistica che manifesta nel suo autore più di un'intenzione involutiva. Nel Système serpeggia una tentazione di allontanarsi dalla tradizione filosofica del XVIII secolo così insistita, da riuscire un po' sorprendente in colui che un paio di decenni prima poteva essere indicato come il maggior continuatore del razionalismo illuminista, con la sua aspirazione a estendere $\mathrm{i}$ processi della ragione dalle scienze della natura all'esperienza umana. Ma Comte ci tiene ora a ribadire che il dogma fondamentale del positivismo è la preponderanza continua del cuore sullo spirito, e preferisce, anziché esaltare le potenzialità della ragione, mettere in guardia sui suoi possibili abusi: "In fondo, le superbe aspirazioni dell'intelligenza alla dominazione universale, dopo che la grande unità teologica si è irrevocabilmente spezzata, non hanno potuto comportare alcuna realizzazione, e non erano suscettibili che di efficacia insurrezionale contro un regime divenuto retrogrado. Lo spirito non è destinato a regnare, ma a servire: quando crede di dominare, rientra al servizio dell'individualismo, anziché favorire la sociabilità, senza che possa in alcun modo evitare di assecondare qualche passione"13.

Il passo riecheggia i giudizi già espressi dal Cours intorno alla Rivoluzione francese, trasferimento alla lotta politica di idee astratte (come libertà e uguaglianza) utili sul piano storico per accelerare la dissoluzione dell' antico regime e del suo teologismo ormai superato, ma inutilizzabili per costruire un nuovo ordine sociale. Sotto accusa, tuttavia, non sembrano essere più soltanto i rivoluzionari in senso stretto, ma anche le loro fonti di ispirazione intellettuale. Respingendo, o quantomeno circondando di enormi cautele i richiami all'esprit (a una parola così cara, come si sa, a Montesquieu o a Condorcet), Comte decide di porsi su un altro piano rispetto alla cultura illuminista. E non per caso fa

\footnotetext{
${ }^{12}$ Comte esprime con queste parole il senso ultimo dell'operazione positivista di ingabbiamento dello spirito per mezzo del cuore: "La costruzione della base oggettiva indispensabile alla grande sintesi umana sconta una difficoltà molto grave, per conciliare la libertà abituale, senza la quale l'intelligenza non potrebbe procedere convenientemente, con la continua disciplina richiesta dalla sua tendenza spontanea alle infinite divagazioni. Questa conciliazione era essenzialmente impossibile, quando lo studio dell'ordine naturale non si estendeva fino alle leggi sociologiche. Ma ora che lo spirito positivo abbraccia realmente questa attribuzione finale, la supremazia necessaria di tali speculazioni sottomette senza sforzo lo studio al giogo del sentimento" (ivi, pp. 36-37).

${ }^{13}$ Ivi, p. 16.
} 
riferimento a un termine religiosamente connotato come dogma, per significare il suo spostamento di ruolo da interprete a costruttore della realtà.

Il rovesciamento di peso fra "cuore" e "spirito" negli scritti comtiani è stato variamente commentato dalla critica. Alle spiegazioni littréane possiamo aggiungere, dopo un secolo e più di ulteriori ricerche sul positivismo e sul suo fondatore $^{14}$, le seduzioni della cultura romantica, portata appunto a valorizzare il peso degli atteggiamenti emotivi nelle esperienze dei singoli e dei popoli. E dobbiamo porre nella giusta luce un'intuizione comtiana davvero notevole per $\mathrm{i}$ tempi, per quanto non espressa in tutti i suoi risvolti, come avrebbe del resto potuto fare solo un uomo del Novecento: l'intuizione cioè che la politica, in un'età di emersione delle pulsioni di massa, si gioca non solo sul piano dei discorsi razionali, ma anche su quello delle rappresentazioni affettive e delle figure sintetiche. Comte vuole parlare al popolo, e sa che il "popolo" (inteso come classi subalterne di limitata acculturazione) segue più le immagini coinvolgenti e passionali (di entusiasmo o di paura) che $\mathrm{i}$ freddi ragionamenti ${ }^{15}$.

A tutto ciò si deve accompagnare, per comprendere la svolta religiosa di Comte, un riferimento al contesto culturale da cui scaturisce il positivismo. L'ambiente di formazione, nonostante il distacco di Comte, è pur sempre quello sansimoniano, nel quale era stata forte e costante l'aspirazione a una ridefinizione del sacro, se non alla fondazione di nuove chiese. Si pensi in particolare al gruppo di sansimoniani raccolti attorno a Enfantin, sospesi tra la fedeltà ai canoni austeri del "nuovo cristianesimo" di Saint-Simon e il desiderio di esprimersi attraverso una liturgia più enfatica. Ma un po' tutta la storia del sansimonismo può essere scritta come farsi e disfarsi di sette, come attrazione e repulsione fra spiriti inquietamente religiosi, fra cui personaggi di spicco come Leroux o Buchez. L'aspirazione a una nuova dimensione del sacro non è del resto esclusiva della scuola sansimoniana,

\footnotetext{
${ }^{14}$ Colgo l'occasione per citare alcuni contributi recenti sul pensiero di Comte, oltre a quello già ricordato di Mirella Larizza: P. Macherey, Comte. La philosophie et les sciences, Paris, PUF, 1989; M. Pickering, Auguste Comte. An Intellectual Biography, Cambridge, Cambridge University Press, 1993; J. Grange, La philosophie d'Auguste Comte. Science, politique, religion, Paris, PUF, 1996. Uno sguardo d'insieme sulla cultura comtiana e su talune sue continuazioni ottocentesche, frutto del contributo congiunto di studiosi italiani e francesi, è inoltre costituito dal volume Sociologia, politica e religione: la filosofia di Comte per il diciannovesimo secolo, a cura di C. Cassina, Pisa, Edizioni Plus, 2001.

${ }^{15}$ Significativo, a questo proposito, è il passo del Système in cui Comte affida maggiore importanza all'arte rispetto alla scienza, nelle strategie di espansione dei precetti della religione dell'Umanità: "L'arte corrisponde meglio della scienza ai nostri bisogni più intimi. Essa è alla volta più simpatetica e più sintetica. Nello stesso tempo respinge con continuità lo stato puramente speculativo, e tende direttamente verso l'azione più nobile, consistente nel perfezionare i nostri sentimenti secondo la loro idealizzazione" (A. Comte, Système, cit., t. IV, p. 51). Lo stesso Comte spiega che, essendo inevitabile nella scienza il pericolo di un'esaltazione eccessiva della ragione umana, la religione deve limitarla al suo ufficio (la conoscenza teorica dell'insieme) e contenerne le possibili deviazioni mediante l'arte. Fra le conseguenze di questo atteggiamento, tutt'altro che indifferenti per il discorso sull'ultimo Comte che stiamo qui sviluppando, vi sono da un lato la convinzione che solo nella morale la scienza ritrova la sua giusta collocazione, dall'altro l'auspicio che nell'educazione positiva conti più l'estetica che la filosofia.
} 
intorno alla metà dell'Ottocento. Una vaga religiosità sorregge anche l'idea di armonia universale degli epigoni di Fourier; a un senso rispettoso per il mistero soprannaturale perviene progressivamente Proudhon; e lo stesso positivismo, anche nelle sue fasi mature e meno comtiane, continuerà a lungo a diffondere, insieme con atteggiamenti scientisti, anche un senso dell'infinito della natura non privo di toni misticheggianti, tali da coinvolgere anche personaggi per molti altri versi antireligiosi (un nome per tutti: Blanqui) ${ }^{16}$.

Ma sul culto e sul dogma comtiani converrà ritornare. Per ora è importante sottolineare che la circospezione distribuita attorno alla ragione e ai suoi pericoli sembra a Comte funzionale all'obiettivo che più gli sta a cuore, quello del raggiungimento di una stabile socialità: e la sociabilità, l'arte di rendere gli uomini solidali, richiede il dispiegarsi di un messaggio non più scientifico, ma direttamente etico. Partito, nel Système, con l'obiettivo di far trionfare il cuore sullo spirito, Comte chiude la sua seconda grande opera con un'interpretazione del positivismo come dottrina morale talmente netta da non lasciar dubbi sulle sue autentiche intenzioni. Tirando le fila del suo lungo discorso, nella conclusione generale del Système egli ripropone lo scopo del suo lavoro intellettuale nelle diverse fasi in questo modo: dapprima (fino al 1847) la trasformazione della scienza in filosofia, poi la trasformazione della filosofia in una religione che nel progetto morale trova la sua giustificazione essenziale. "L'intima connessione fra le due fasi della mia missione risulta dall'obbligo di rendere completa l'unità positiva, affinché essa sia efficace e durevole. Se le teorie scientifiche rimangono insufficienti e precarie senza un legame filosofico, lo stesso motivo esige che questo si subordini al principio morale, sola fonte della sintesi soggettiva, necessariamente indivisibile" ${ }^{, 17}$.

La delineazione del discorso morale di Comte richiede una precisazione introduttiva. Il fondatore del positivismo scrive il Système qualche decennio dopo la formulazione della filosofia hegeliana, che conosce abbastanza bene. E quindi sa

\footnotetext{
${ }^{16}$ Se è lecito aggiungere a questa un po' bizzarra compagnia un rinvio a filosofi serissimi, si potrebbe ricordare che anche una parte considerevole del pensiero di Hegel si muove alla ricerca di una ridefinizione dello spirito di segno immanentistico. E, per rimanere in ambito tedesco e per ricordare un'ulteriore variante dell'ansia di sacro che cerca di travalicare i limiti del cristianesimo tradizionale, anche l'attenzione di Schopenhauer per le religioni orientali può forse essere ricordata in questo contesto.

${ }^{17}$ A. Comte, Système, t. IV, p. 530. Possiamo osservare che l'approdo a un senso prevalentemente morale della propria esperienza aveva caratterizzato anche 1'ultimo Saint-Simon, quello del "nuovo cristianesimo". "Il nuovo cristianesimo, allo stesso modo delle associazioni eretiche, avrà la sua morale, il suo culto e il suo dogma; avrà il suo clero e il suo clero avrà i suoi capi. Tuttavia, nonostante questa somiglianza d'organizzazione, il nuovo cristianesimo sarà purgato da tutte le eresie attuali; la dottrina della morale sarà considerata dai nuovi cristiani la più importante: il culto e il dogma saranno considerati da essi soltanto come accessori, aventi lo scopo principale di fissare sulla morale l'attenzione dei fedeli di tutte le classi" (H. de Saint-Simon, Nuovo cristianesimo, a cura di G.M. Bravo, Roma, Editori Riuniti, 1974, p. 11). Comte avrebbe potuto sottoscrivere quasi interamente il contenuto di questo passo, a eccezione forse, come vedremo a proposito della religione dell'Umanità, della riduzione di importanza del culto, per lui invece essenziale per il raggiungimento delle finalità morali.
} 
che Hegel aveva distinto concettualmente la morale individuale dall'etica. Come è noto, per il filosofo tedesco la morale individuale, l'aspirazione soggettiva a comportamenti giusti, è cosa diversa, e molto più incerta, rispetto all'estrinsecazione oggettiva dell'idea di bene racchiusa nel termine di etica, che ha i suoi ambiti di concretezza nelle formazioni storiche della famiglia, della società civile e dello stato. Comte, come vedremo meglio, continua invece a sovrapporre morale soggettiva ed eticità. Da un lato si muove anch'egli alla ricerca dei "luoghi" in cui la storia concentra le regole di riconoscimento comune intorno alla bontà dei comportamenti umani, ma fa più fatica di Hegel a identificarli con chiarezza: se possiede anch'egli una dottrina della famiglia, non sa precisare le distinzioni fra società e stato, augurandosi in fondo, sansimonianamente, la riduzione delle funzioni di quest'ultimo a organo programmatore per la società industriale. È vero che l'ultimo Comte presta attenzione al motivo della patria, il che potrebbe far pensare, attraverso la dimensione implicita della nazione, a un rinvio alla sfera statale; ma lo stadio della patria rimane in lui sempre oscurato dall' ampio, e perciò generico, riferimento alla grande sfera dell'umanità. D'altro lato, il fondatore del positivismo continua a guardare con interesse e fiducia alla morale individuale, alla quale fa appello perché ispiri l'adesione volontaria agli scopi comuni della specie: un auspicio di mobilitazione delle singole coscienze dal quale dipende, fra l'altro, la crescente propensione comtiana per lo sviluppo di un discorso dichiaratamente religioso. Occorre aggiungere che, in questa sovrapposizione di significati, Comte, e i positivisti nel loro complesso, non sono aiutati dalla lingua. Nel francese del tempo, anche in quello colto, la diffusione del termine morale, rispetto a éthique, è di gran lunga prevalente. Ed è perciò quasi inevitabile che le varie accezioni riguardanti il giudizio di valore sui comportamenti pratici degli uomini siano etichettate sotto un'unica voce, appunto la "morale".

La morale ha nel pensiero comtiano la funzione di esaltare i legami di unità fra individuo e umanità, affinché ognuno anteponga alle proprie aspirazioni personali la coesione della specie e il suo perfezionamento etico. Il luogo iniziale in cui ciò avviene è la famiglia, centro di affetti naturali in cui l'individuo impara a smussare le proprie tendenze egoistiche per riconoscersi in rapporti sentimentali socializzati (coniugali, materni, paterni, filiali, fraterni). La teoria positiva della famiglia, avanzata da Comte in particolare nel secondo tomo del Système, non ne fa solo il luogo della riproduzione, pure indispensabile alla continuità umana, ma anche della soddisfazione sessuale connessa all'amore e al legame di solidarietà fra i coniugi. La vera morale familiare trova anzi alimento prima nell'incontro fra gli sposi, e solo successivamente nella rete di rapporti costruita con la paternità e la maternità, che va dall'amore dei genitori per i figli a quello di questi per chi li ha generati, fino alla collaborazione insita nel motivo della fratellanza.

Il positivismo non si accontenta tuttavia di disegnare come fondamentali i legami di affetto solidale che nascono all'interno delle famiglie. Occorre anche che le famiglie non si chiudano in se stesse, ma si aprano alla società con un atteggiamento morale analogo a quello che si deve costruire al loro interno. 
Tracciando una vera e propria teoria politica della famiglia, Comte la identifica come il microcosmo in cui si manifestano gli elementi di amore, di fede e di attività che devono caratterizzare l'intera società. Il modello di articolazione fra potere materiale e potere spirituale su cui si regge l'idea di società positiva, trova d'altro canto origine nei ruoli che si instaurano in una famiglia fra $i$ due coniugi, all'interno di una relazione (monogamica, aggiunge Comte, essendo questa la sola adatta allo spirito positivo ${ }^{18}$ ) basata sull'amore. Il marito e padre raffigura l'autorità all'interno della famiglia e insieme il nesso principale con la società per mezzo del lavoro esterno. Nelle attese comtiane il lavoro deve infatti essere esclusiva dell'uomo, che fra i suoi doveri ha quello di mantenere la donna. La donna non ha tuttavia un'esistenza solamente privata: se non produce, ha comunque un ruolo pubblico di diffusione della morale positiva, la cui sistemazione definitiva è però opera dei filosofi.

Se per il positivismo è comprensibile che l'uomo abbia in famiglia un ruolo preminente, anche se in realtà dovrebbe consigliare più che comandare, sul piano educativo e morale la funzione principale è incarnata dalla donna, il soggetto più adatto per natura a trasmettere $\mathrm{i}$ valori di amore universale, visto che li pratica spontaneamente a partire dall'atto della riproduzione. Per Comte tutto ciò non è una concessione a una specie di tradizionalismo familista (che pure ispira molte delle sue posizioni conclusive), ma la costruzione di un modello pronto a permeare di sé tutta la politica positiva. "Se la costituzione domestica si riduce alla sistematizzazione dell'influenza della donna sull'uomo, si può dire ugualmente che la costituzione politica consiste soprattutto nel regolare l'azione del potere intellettuale sulla potenza materiale, ${ }^{, 19}$.

Sarebbe un errore di prospettiva se, nei molti apparentamenti costruiti nel Système de politique positive fra dimensione familiare e sistemazione sociale, volessimo trovare niente più della continuità di una metafora ormai convenzionale nel pensiero politico, quella dello stato come grande famiglia. Comte si muove invece alla ricerca di un significato meno descrittivo e molto più impegnativo, quello della costruzione di una società politica ideale che prende per base le pulsioni affettive della famiglia ideale. La stessa organizzazione gerarchica della società, con le distinzioni fra patrizi e proletari, non deriva solo da un bisogno di autorità, ma persegue anche l'amore fra classi necessariamente differenti, perché la disuguaglianza è insita nella natura e nella storia, ma proiettate verso un affetto analogo a quello che, fra diversi, si dovrebbe respirare in famiglia.

La morale, se parte da legami naturali, non si esaurisce peraltro nella spontaneità, ma richiede un forte processo educativo, di cui il positivismo si fa carico come qualsiasi altra etica precedente. Primo luogo dell'educazione è ancora

\footnotetext{
${ }^{18}$ La famiglia monogamica non esclude peraltro il divorzio, di cui Comte prevede la possibilità, anche se non si dilunga sulle ragioni che possano giustificarlo. La scelta per un solo rapporto coniugale che duri per tutta la vita resta comunque fonte di merito: lo si desume anche dal fatto che, dopo la morte del coniuge, la società positiva prevede l'obbligo della vedovanza, salvo dispensa che solo il capo della chiesa positiva potrà concedere.

${ }^{19}$ A. Comte, Système, cit., t. II, p. 209.
} 
una volta la famiglia, nella quale si esalta appunto il ruolo ammaestratore della donna. Più spontanea che sistematica, essendo del resto le donne le "sacerdotesse spontanee dell'Umanità"20, l'educazione familiare usa congiuntamente l'esercizio fisico e l'apprendimento estetico (poesia, musica, pittura) affinché le credenze collettive emergano e si sviluppino in forme per così dire naturali. Degno di attenzione è il fatto che l'educazione religiosa in ambito domestico, quando si rivolge ai più giovani, non deve essere troppo apertamente prescrittiva a favore del culto positivo dell'Umanità. Nella sua esistenza individuale è anzi bene che ciascuno ripeta in piccolo l'evoluzione che il genere umano ha attraversato in grande, arrivando allo stadio positivo dopo aver praticato e concepito le fasi del feticismo, del politeismo e del monoteismo ${ }^{21}$.

Come la morale familiare è destinata a prolungarsi nella morale comunitaria, così l'educazione domestica è da un certo grado in avanti sollecitata a saldarsi all'educazione pubblica. Il soggetto dominante diventa, in questo secondo caso, il potere spirituale, cioè l'insieme dei filosofi positivisti, ai quali spetta di elaborare la dottrina sistematica della sociabilità, di diffonderla nel corpo sociale e di farne uno strumento di controllo del potere materiale. Nella loro predicazione sociale i filosofi sono sicuri di trovare degli alleati nelle donne (per tutto quello che si è detto finora) e nei proletari, i destinatari principali degli atti di governo del potere materiale. Questo va affidato per ragioni di competenza al patriziato industriale, il quale è però obbligato a elevare il livello di vita dei proletari, assicurando loro il lavoro alle migliori condizioni possibili. I proletari potranno essere all'occorrenza i maggiori alleati del potere spirituale, per definizione retto, quando fosse necessario impedire al potere materiale di deviare dai suoi fini solidali.

L'educazione pubblica, come in tutti i grandi progetti di moralizzazione collettiva, dovrebbe estendersi, nelle aspettative comtiane, all'insieme degli elementi della convivenza sociale, specialmente nelle sue forme culturali. Luogo privilegiato della trasmissione educativa sarà ovviamente la scuola, per la quale i positivisti, soprattutto attorno al 1848 , richiedono una riforma che tolga l'istruzione da un lato all'influenza teologica della chiesa cattolica, dall'altro allo scetticismo metafisico delle accademie. Ma il processo educativo deve coinvolgere tutti, non solo i giovani: da qui l'attenzione di Comte per una estesa rete di comunicazioni culturali di vario genere e intensità, che va dalla formazione di una biblioteca

\footnotetext{
${ }^{20}$ Ivi, t. I, p. 227.

${ }^{21}$ Ricordo, anche se fa parte degli aspetti più frequentati del pensiero di Comte, che già nel Cours lo stadio teologico veniva riarticolato in tre momenti. Il primo è quello feticista, il più antico negli atteggiamenti religiosi dell'umanità, in cui l'accostamento al sacro avviene attraverso la divinizzazione di elementi o fenomeni naturali. Il secondo è quello politeista, proprio in particolare delle grandi civiltà urbane dell'antichità, nel quale il discorso religioso si basa sul riferimento a più divinità, spesso pensate in termini antropomorfi. Il terzo, proprio soprattutto della tradizione giudaicocristiana, è quello monoteista, in cui rimangono aspetti dell'antropomorfismo precedente nella rappresentazione del sacro (l'uomo a immagine e somiglianza di Dio, il Figlio di Dio che si fa uomo), anche se il passaggio da molti dei a un solo Dio avvicina la teologia al predominio delle idee astratte che si registrerà nello stadio metafisico.
} 
positivista per l'acculturazione dei ceti alfabetizzati fino al dispiegamento di iniziative di culto e di festa in nome dell'Umanità.

Sul vasto impiego di riti e simboli nella società positiva, torneremo a proposito dei caratteri costitutivi della religione dell'Umanità. Quanto alla biblioteca positivista, vale forse la pena seguire per un attimo la selezione proposta da Comte delle letture ideali per un positivista ${ }^{22}$. La scelta è vasta (centocinquanta volumi, per un numero ancora più elevato di titoli, visto che molti singoli volumi sono pensati come raccolte o sintesi di più libri), e perciò indicativa della coscienza nel suo propositore del bisogno di una pluralità di fonti culturali. Degna di interesse è inoltre l'operazione in sé di delineare un modello compiuto di letture, e nell'offrirne un'immagine sistematica basata sulla compresenza di opere di letteratura, scienza, storia e sintesi (parola con cui Comte intende le letture atte alla ricostruzione dei fondamenti della filosofia positiva). Al di là dei titoli e degli autori citati, che presentano comunque qualche tratto di curiosità ${ }^{23}$, quel che è

${ }^{22}$ L'elenco dei titoli che devono figurare nella biblioteca positivista appare per la prima volta in appendice all'edizione del 1851 del Catéchisme positiviste, e trova posto definitivo nel IV tomo del Système, alle pp. 557-561. W.M. Simon, in Il positivismo europeo nel XIX secolo (Bologna, Il Mulino, 1980, p. 87), ricorda che nel 1880 fu effettivamente aperta a Parigi una biblioteca positivista con i titoli previsti da Comte.

${ }^{23}$ L'impostazione culturale che Comte vuole conferire alle letture del positivista militante appare soprattutto nell'ambito letterario, con la sua fusione fra la cultura classica e quelle nazionali dell'Europa occidentale, filtrata alla luce di un gusto in prevalenza romantico. Oltre agli immancabili "classici" (da Omero ai tragediografi greci, da Virgilio agli altri poeti latini), appaiono nell'elenco le tradizioni nazionali italiana (Dante, Petrarca e altri), spagnola (Cervantes), francese (con una vasta rappresentanza sei-settecentesca, da Corneille e Molière a La Fontaine e Voltaire), inglese (Shakespeare). Fra i romanzieri moderni spicca per quantità (sette titoli) la presenza di Walter Scott, ma non mancano altre opere di intrattenimento avventuroso e insieme edificante della letteratura inglese del secolo precedente, da Robinson Crusoe al Vicario di Wakefield. Da segnalare è la citazione dei Promessi Sposi, che si conferma come uno dei titoli della letteratura di età romantica più apprezzati su scala europea. Più tecnica è naturalmente la scelta riguardante la scienza, che riflette la classificazione comtiana delineata già nel Cours. Vi appaiono infatti opere di matematica (da Cartesio a Condorcet a Legendre), di astronomia, di meccanica e fisica, di chimica (con la citazione di scienziati importanti come Lavoisier e Berthollet), di biologia (da Buffon a Lamarck, fino a uno scienziato vicino al positivismo come Bernard). Da ricordare è il fatto che Comte pone anche due suoi scritti fra i libri-base di argomento scientifico: quelli riguardanti la geometria analitica e l'astronomia popolare. Il carattere celebrativo e non solo esplicativo che deve assumere la scienza nel costume contemporaneo è implicitamente sottolineato dalla presenza degli elogi dei savants composti nel Settecento per l'Accademia delle Scienze da Fontenelle e da Condorcet. A metà fra interesse scientifico e piglio letterario è la scelta delle opere di storia. Indicazioni impegnative di letture storiografiche (dal Bossuet del compendio di storia francese a Voltaire, da Robertson a Hume a Gibbon) sono bilanciate dal rinvio a una memorialistica di taglio più narrativo (come i resoconti di viaggio di Cook e Chardin, o le memorie di Benvenuto Cellini), o a testi di più marcato significato politico, come il testamento di Richelieu. Da segnalare sono la presenza di una sola storia della Rivoluzione francese, quella filogiacobina di Mignet, e l'interessante considerazione riservata alla storia dell'arte di Winckelmann, al trattato di pittura di Leonardo e alle memorie sulla musica di Grétry, spia del modo esteso di intendere la cultura proprio di Comte. Non mancano poi i rinvii alle opere di argomento storico della classicità, con un rilievo particolare accordato a due autori di forte impegno morale come Tacito e Plutarco. Fra le opere di sintesi, una parte rilevante è significativamente occupata dai testi fondamentali di qualsiasi biblioteca confessionale: Bibbia e 
significativo è che Comte ripercorra una strada che prima di lui è appartenuta soprattutto alla letteratura a sfondo utopico: l'intenzione, cioè, o la presunzione, di voler indicare un corpo completo di letture "appropriate", una biblioteca appunto ideale, come ideale è la società complessiva tratteggiata dalle utopie.

\subsection{Verso un'utopia conservatrice}

A proposito della biblioteca positivista si è appena fatto cenno alla presenza, nell'ultima fase dell'opera comtiana, di suggestioni derivanti dai procedimenti della letteratura utopistica. L'analogia non si ferma tuttavia a pochi dati esteriori. È l'incombenza stessa di un rigido progetto etico, che intende informare di sé tutte le manifestazioni della vita personale e collettiva, che finisce per avvicinare il Système de politique positive a un'opera utopica, alla quale mancano certo viaggi immaginari e isole più o meno misteriose, ma non fa difetto lo scopo principale di ogni utopia precedente: la rifondazione morale del mondo. Comte si dimostra anzi aggiornato su questo punto: in modo non dissimile dal suo vecchio maestro SaintSimon, ma anche da un utopista dai caratteri ancor più marcati come Fourier, egli non affida la felicità morale a un'esistenza segnata da un'accentuata frugalità, ma cerca di coniugare la perfezione morale con l'abbondanza materiale, o comunque con una vita fatta di soddisfazioni non solo culturali.

Che si possa interpretare l'ultima grande opera comtiana facendo riferimento alle categorie dell'utopismo è giustificato da alcuni passaggi testuali dello stesso Comte e da alcune figure specifiche presenti nel suo libro. Inoltre, come vedremo, è giustificato dalle strategie di fondo dell'argomentazione comtiana, volte a rappresentare il potere politico come ancorato a un' architettura sociale ideale, più immaginata che ricavata dalla realtà effettiva. In un punto del IV tomo del Système, quando parla della completa realizzazione del regime positivo, Comte evoca a più riprese la parola utopie, riprendendo un'affermazione già posta all'inizio dell'opera, quella del positivismo come nuova utopia che supera le insufficienze e i fallimenti delle utopie precedenti, alle quali mancavano le consapevolezze della scienza sociale. Scrive Comte, ricordando uno degli obiettivi fondamentali del positivismo, la composizione fra ordine e progresso: "Bisogna che la conclusione sintetica concili l'ordine e il progresso, istituendo un progresso che sviluppi l'insieme dell'ordine. Ora, questa è l'attitudine delle utopie convenientemente costruite, il cui sviluppo crescente ha indicato, senza soddisfarlo, il bisogno di unità

Corano, Sant'Agostino e San Bernardo, catechismi e tanto Bossuet, fino a de Maistre. Il bilanciamento è offerto da indicazioni di letture filosofiche che privilegiano gli autori cui Comte ha già fatto riferimento dai tempi del Cours, non di rado di atteggiamento laico come Hume, Diderot, Condorcet, Cabanis. A rappresentare la filosofia greca è Aristotele, primo teorizzatore della socialità naturale degli uomini, ma non Platone. Il punto d'arrivo è naturalmente l'opera di Comte, vicina a essere indicata come opera omnia: e se curiosamente la filosofia positiva si può apprendere, per autorizzazione del suo stesso autore, attraverso l'edizione condensata proposta per il pubblico inglese da Harriet Martineau, la politica positiva e il catechismo positivista devono apparire integralmente nella biblioteca ideale. 
sorto dall'anarchia moderna". E prosegue: "Il dominio finale della positività, sia ideale sia reale, esige una dottrina universale, senza cui l'utopia, che deve costituirne una sintesi, potrebbe diventare soltanto un'intuizione più perturbatrice che feconda. Questa condizione fondamentale si trova tuttavia sufficientemente soddisfatta dopo l'avvento decisivo della sociologia, da cui risulta l'irrevocabile convergenza della rivoluzione occidentale con lo stabilirsi della religione positiva" ${ }^{24}$. Come avviene spesso in Comte, anche a proposito della dimensione utopica assistiamo a un avvicinamento duplice, favorevole e diffidente insieme. In quanto costruzioni puramente mentali, le utopie possono essere frutto di sterili artifici intellettuali. Ma l'aspirazione all'armonia universale da esse in qualche modo rappresentata rimane integra anche per il positivismo, che vuole riprendere l'impianto tradizionale delle utopie per trasferirle su un terreno di più immediato coinvolgimento emotivo, anziché razionale.

Si può aggiungere che, per quanto usi indistintamente il termine di utopia, Comte si muove a volte alla ricerca di veri e propri miti, capaci di illustrare sinteticamente i desideri profondi del positivismo. Una delle figure più disarmanti (provenendo da un sostenitore del progresso scientifico) è quella che egli chiama l'utopia della Vergine Madre. Si tratta di una figura esterna alle coordinate razionali, ripresa ovviamente dalla tradizione teologica cristiana, che l'autore del Système ripropone come mito "eterno" della procreazione unita a castità. È intuitivo come tale condizione appaia irrealizzabile, frutto di immaginazione, da cui la definizione di utopica: ma il positivismo, con la sua fiducia nel progresso scientifico e morale, lascia aperta l'ipotesi che il progredire delle scienze biologiche e mediche possa renderla realizzabile in futuro. E il moralista Comte, impegnato in un'esaltazione mistica dell'amore angelicato per Clotilde de Vaux, trova in questa ipotesi qualche motivo per rovesciare il precedente occhio favorevole posto sulla soddisfazione erotica all'interno del matrimonio. L'utopia della Vergine Madre è un'immagine sintetica che permette di pensare al bene della continuazione della specie disgiunto dai mali della sessualità (compresi i relativi rischi di contrarre malattie).

L'utopismo rintracciabile nell'ultima opera comtiana non si ritrova soltanto quando il suo autore ripercorre esplicitamente itinerari propri della tradizione del genere, ma anche quando la sua argomentazione complessiva fa ricorso in forme implicite alle tecniche dell'immaginazione sociale. Tale è in larga parte il discorso sulla società positiva, nella sua strutturazione di fondo dominata da impulsi etici, nella puntigliosa ricostruzione dei ruoli sociali al suo interno, nell'auspicata fissità e armonia di questi ruoli, nella presenza di una vasta ritualità civile destinata a conferire sacralità ai legami sociali. Non è qui possibile ricostruire analiticamente le componenti della politica positiva, un lavoro che fra l'altro ho già cercato di fare altrove, come analisi degli elementi istituzionali e politici che vengono a comporre

\footnotetext{
${ }^{24}$ A. Comte, Système, cit., t. IV, p, 274.
} 
il rapporto fra potere e classi sociali nel regime positivo ${ }^{25}$. Mi limito a ricordare che la posizione politica di Comte risulta difficilmente catalogabile, se non addirittura sfuggente, già dai tempi del Cours. Da un lato si respira infatti, nella sua concezione di repubblica, un'aria di autoritarismo talvolta pesante, sorretto da una fiducia incrollabile che la verità scientifica diventi fondamento e guida del governo, al quale non possa opporsi un dissenso "razionale". Spie di queste tensioni autoritarie sono le frequentazioni dei testi di Bossuet e di de Maistre, eccessive da parte di uno che continua su un altro versante a definirsi un combattente per il progresso, per quanto temperato dal richiamo all'ordine. Ma c'è d'altro lato un Comte più aperto alle sollecitazioni che provengono dalla sinistra dello schieramento politico del suo tempo. Penso al Comte "giacobino", che vede nel centralismo la fine delle differenze arcaiche ereditate dal passato e l'inizio di una politica uniformatrice; al Comte che ravvisa nel sistema industriale il mezzo principale per costruire pacificamente il mondo del futuro; al Comte che all'interno di questo sistema ritaglia uno spazio fondamentale per i bisogni del proletariato, a partire dal diritto al lavoro; al Comte che, se continua a polemizzare con i princìpi dell' 89 giudicandoli astratti alla moda dei controrivoluzionari, comprende tuttavia la Rivoluzione sul piano storico. Si tratta certo di pulsioni e atteggiamenti ambigui, non privi di elementi contraddittori: ed è proprio per questo, forse, che il Système cerca di offrirne un'immagine più armonica, facendo riferimento a più riprese all' argomentazione utopica.

Ammesso che Comte affronti consapevolmente questo passaggio alla dimensione dell'utopismo (e io ne sono convinto), resta peraltro evidente che si tratta di un'utopia dai caratteri particolari, soprattutto perché non evita, a differenza delle utopie delle "isole felici", di fare i conti con la storia. Era già accaduto, prima di Comte, a Condorcet, il quale aveva trovato in un'immagine radiosa del futuro, al termine dell'Esquisse sul progresso storico dell'umanità, la compensazione idealizzata ma non improbabile alle difficoltà della storia reale. Condorcet si era tuttavia accontentato di delineare i tratti portanti del futuro (uguaglianza fra le nazioni, minore disuguaglianza fra gli individui, perfezionamento della specie), senza preventivarne in forme assolute le modalità di attuazione, senza in altri termini voler prescrivere con un atto di autorità intellettuale la vita degli uomini fin nei minimi particolari, come è proprio invece delle utopie "forti". Ed era rimasto fedele a un'interpretazione progressiva della storia, senza imbarazzi nel giudicare il passato meno evoluto del presente (e soprattutto del futuro) e senza ritagliare spazi positivi per la religione, da sostituire con una laica fiducia nelle potenzialità indefinitamente crescenti della ragione. Comte percorre al contrario una strada molto più vicina alle utopie in senso stretto: si incarica di descrivere la società

\footnotetext{
${ }^{25} \mathrm{Mi}$ riferisco a due miei contributi apparsi in opere collettive: Politica positivista e governo repubblicano: da Comte a Littré, in Il popolo e le élites. Tra Romanticismo e Positivismo nella Francia dell'Ottocento, a cura di V. Collina, Milano, Mimesis, 1997; e La repubblica positiva da Comte a Littré, in Sociologia, politica e religione: la filosofia di Comte per il diciannovesimo secolo, cit.
} 
ideale fin nelle sue minime articolazioni (soprattutto in merito alla morale e alla religione), rendendo la sua costruzione statica, data una volta per tutte e alla fine sottratta alla storia.

Nell'ambito del Système questo atteggiamento comtiano risalta soprattutto a proposito delle nuove gerarchie instaurate fra ordine e progresso. Come si sa, l'aspirazione a coniugare armonicamente ordine e progresso, togliendo il primo termine alla gestione delle forze puramente retrograde e il secondo al dominio di quelle puramente rivoluzionarie, era uno dei caratteri più tipici del positivismo fin dalle sue prime mosse. Ciò non escludeva tuttavia che il messaggio di Comte racchiuso nel Cours fosse accolto, per esempio da Littré ma non solo da lui, soprattutto come una dottrina del progresso, per quanto sorvegliata appunto da una propensione a ridurre al minimo gli effetti destabilizzanti insiti nella trasformazione storica. Più tecnicamente e in termini all'inizio più descrittivi che propositivi, la distinzione fra ordine e progresso si era venuta configurando, all'interno del linguaggio comtiano, come articolazione fra statica sociale e dinamica sociale. Nella prima formula sono compresi gli elementi che caratterizzano la società delle origini ma non possono mancare in nessuna aggregazione umana, per quanto progredita, finendo così per illustrare l'idea di società in quanto tale ${ }^{26}$. La dinamica si incarica invece di determinare le linee dello sviluppo passato e futuro dell'umanità, attraverso la scoperta e la descrizione delle leggi con cui si manifesta il progresso.

Se la dinamica sociale appariva a molti commentatori come il segno prevalente dell'argomentazione del Cours, nel Système emerge maggiormente il peso della statica, in sintonia col rovesciamento di gerarchie fra il cuore e lo spirito. All'inizio del terzo tomo dell'opera, dedicato appunto alla teoria generale del progresso umano, Comte ribadisce più volte che le nozioni del progresso vanno subordinate alle leggi della statica: le quali ci dicono che l'umanità è destinata ad avvicinarsi all'unità e che, per non attardarsi in inutili e costosi rallentamenti su questo cammino, vanno rinforzati gli elementi di coordinazione soffocando gli impulsi individuali. Fattori principali del progresso sono l'evoluzione teorica e lo sviluppo pratico. Se sul primo versante l'avvento della filosofia positiva rende Comte ragionevolmente ottimista sulla possibilità di far trionfare i legami di solidarietà, sul piano pratico della vita economica delle società il motivo del perfezionamento richiede un ancor più forte appello alla mobilitazione comunitaria, al fine di impedire l'emergere dei particolarismi. Il progresso va quindi reinterpretato soprattutto come sviluppo dell'ordine, riconoscendo preminenza all'aspetto oggettivo dell'evoluzione storica, anziché a quello soggettivo. La rappresentazione

\footnotetext{
${ }^{26}$ Se dovessi riassumere gli elementi propri di ogni società secondo Comte (e che quindi dobbiamo ritrovare, perfezionati, anche nello stadio positivo), direi che si tratta dei seguenti: la propensione spontanea dell'uomo alla socialità, rafforzata dalle istituzioni; la conformazione del gruppo sociale come articolato in famiglie (e non in individui); la necessità di un governo che distribuisca i compiti produttivi tra le varie famiglie; la persistenza di una credenza comune che disegni come sacri i vincoli di unità sociale; la conseguente formazione, analoga a quanto avviene per il governo in ambito materiale, di un nucleo di sapienti con il compito di delineare e diffondere la verità morale.
} 
dell'ordine deve di conseguenza uniformarsi a un'immagine di unità non come scopo prodotto dalla ragione, ma come forza immanente che dà senso a tutta la storia, e che può essere comunicata solo facendo appello alla religione dell'Umanità. Tale circolarità di argomenti viene a un certo punto espressa da Comte in termini categorici: "Tutto lo studio sistematico dell'evoluzione umana deve consistere nello sviluppo della sua unica legge: l'uomo diviene sempre più religioso. La dinamica sociale si riduce in effetti alla spiegazione del passato sulla base di questo principio fondamentale, emanato dalla sociologia statica"27.

Questa circolarità concettuale si riflette in altri tipi di involuzione presenti nella "storiografia" comtiana, oscillante fra dottrina del progresso e indugio sulla valorizzazione del passato. La rivalutazione del passato non è solo un mezzo per rendersi conto dell'effettivo svolgersi della storia, ma anche una ricerca di esperienze che abbiano qualcosa da suggerire anche alla società positiva, o comunque servano per costruire un'immagine solida di continuità nella storia ${ }^{28}$. Già nel Cours si era per esempio affacciata un'interpretazione tutto sommato positiva del medioevo cristiano, che ritroviamo intatta, e anzi approfondita, nel Système. L'apprezzamento va in questo caso a un periodo in cui si era delineato in modo abbastanza nitido il programma, che è anche del positivismo, di far collaborare potere materiale e potere spirituale sotto l'impulso di una dottrina comune.

Comte riconosce che le realizzazioni erano state per la verità inferiori al modello, da un lato per il persistere di costumi militari inadatti a perseguire il progresso civile, dall'altro per la pretesa del clero di occupare spazio anche nel potere materiale, oltre che in quello spirituale. Ma adeguarsi alla considerazione per il medioevo serve a Comte per collegarsi a un luogo comune della rappresentazione storica romantica, contro i giudizi consolidati della storiografia illuministica. E rende più intelligibile un progetto politico e religioso insieme, che intende guardare al passato come a una fase integrante, inestricabile dal presente e dal futuro, della formazione dell'Umanità come soggetto sacro. In un passo del Système il fondatore del positivismo, descrivendo il suo movimento come seconda e costruttiva fase di una rivoluzione occidentale che nel sommovimento del 1789 ha conosciuto il suo primo e distruttivo momento, caratterizza la funzione del positivismo nella storia facendo appello alla dottrina fondamentale dell'unità, così come viene elaborata dal potere spirituale. E continua: "Lo spirito generale di questa dottrina è principalmente storico, mentre la parte negativa della rivoluzione dovette far prevalere uno spirito antistorico. Un odio cieco contro il passato era allora indispensabile per uscire energicamente dall'antico regime. Oggi, al contrario, la nostra emancipazione finale esige che rendiamo al passato una completa giustizia, che diverrà il tributo più caratteristico del vero spirito positivo,

\footnotetext{
${ }^{27}$ A. Comte, Système, cit., t. III, p. 10.

${ }^{28} \mathrm{Si}$ tenga conto che, come è ribadito in particolare nel preambolo al terzo tomo del Système, è convinzione del positivismo di possedere una filosofia della storia come espressione di uno spirito storico universale.
} 
il solo oggi suscettibile di una simile attitudine, in virtù della sua permanente natura relativa" 29 .

Comte non si limita con questo atteggiamento al condivisibile invito ad avvicinarsi alla materia storica al di fuori di preconcetti spiriti di condanna. Il suo obiettivo è più ambizioso: è quello di innalzare lo svolgimento storico ad argomento religioso, focalizzandolo in un'immagine immanente della divinità, coincidente con l'umanità nel suo cammino da una generazione all'altra, tutte unite nel ruolo, difficilmente componibile in una rappresentazione razionale, di adoratrici di se stesse e di oggetto di adorazione. Quale che sia la plausibilità delle immagini religiose di Comte, resta il fatto che il loro scopo principale è quello di far interpretare le esperienze soggettive come parti di un ordine universale oggettivo. Seppur non nazionalista, Comte finisce su questo punto per portare il suo contributo a quello che diverrà uno dei miti del nazionalismo novecentesco, il mito della comunità di appartenenza come corpo vivente che impegna al rispetto di coloro che ci hanno preceduti e di coloro che ci seguiranno, in quanto membra dello stesso corpo di cui fa parte la generazione presente. "La più grande difficoltà della rigenerazione positivista consiste nel risveglio continuo dell'esistenza soggettiva in tutte le anime. Bisogna che la popolazione attuale si senta sempre collocata fra l'insieme dei suoi predecessori e l'insieme dei suoi successori, al fine di sviluppare la continuità fondamentale che la conduce dagli uni verso gli altri. Dovremo perciò intrattenere con i morti, e anche con i non nati, un rapporto più sistematico, seppur meno speciale, che con i nostri contemporanei. Ora, questa condizione insormontabile diverrebbe impraticabile senza la decisiva preparazione derivata spontaneamente dallo stadio teologico"30. La parte della teologia da recuperare a cui Comte pensa in questo contesto, è quella che ha diffuso l'abitudine a riferirsi a forme di vita ideali, trascendenti l'esistenza soltanto individuale.

\footnotetext{
${ }^{29}$ A. Comte, Système, cit., t. I, p. 85.

${ }^{30}$ Ivi, t. IV, p. 24. Rappresentativi sono anche altri passaggi del Système a questo proposito, soprattutto il luogo in cui Comte ipotizza l'istituzione di un giorno l'anno di glorificazione dei morti, definiti nel loro insieme "dominatori necessari dei viventi" (ivi, p. 153). Definisco Comte "non nazionalista", e credo che questo riconoscimento rimanga valido alla luce dell'idea di alleanza occidentale che continua a ispirare fino al termine l'argomentazione politica del fondatore del positivismo, proiettato verso una dimensione sovranazionale della società positiva. La stessa concezione dell'Umanità come entità sacra, seppur suddivisa in zone geografiche di diverso livello rispetto al progresso storico verso lo stadio positivo, spinge a tale giudizio. L'assunzione di Comte fra i padri ispiratori del nazionalismo francese, operazione tentata soprattutto all'inizio del Novecento, se è giustificata da qualche citazione possibile dalla peraltro immensa opera comtiana (fra cui i passi qui ricordati), mantiene quindi anche un certo carattere di forzatura. Non è tuttavia privo di significato un cambiamento intercorso fra il Cours e il Système. Nella prima opera, come istanza di raccordo politico fra la più circoscritta e la più ampia delle aree di socialità (la famiglia e l'umanità), veniva indicata la Città: dizione peraltro ambigua, che può indicare sia la municipalità in senso stretto sia una più generica cittadinanza intermedia. Nella seconda opera al posto della Città affiora la Patria, più consona a interpretare quel senso di continuità fra passato e presente che ispirerà appunto la cultura nazionalista. Ma "padre" del positivismo non è tanto il passato francese, quanto il passato occidentale, per cui il "patriottismo" comtiano sembra alimentato, più che da un vero e proprio nazionalismo, dalla convinzione della superiorità dell'Occidente.
} 
Il modo con cui l'ultimo Comte guarda all'indietro, all'interno di un progetto di delineazione di condizioni ideali religiosamente vissute, colora insomma la sua utopia di sentimenti conservatori che all'inizio erano meno pressanti nel suo impianto di pensiero. Altri elementi confermano nel Système questo scivolamento di Comte verso la moderazione dei toni progressivi: dall'imporsi di un potente apparato ecclesiale di origine positivista (per quanto più sognato che realizzato) alla ridefinizione dei ruoli sociali volta più a consolidare l'autorità dei ceti dominanti che la funzione progressiva delle rivoluzioni promosse dalle classi subalterne. Resta comunque vivo, in questo ritorno all'ordine, anche qualche richiamo al progresso, per cui possiamo dire che si inaugura con Comte un atteggiamento che sarà proprio di molto conservatorismo europeo di fine Ottocento, quello che continuerà a volersi distinguere dai sentimenti puramente reazionari. Si tratta di un conservatorismo che non vuole tornare all'indietro nella serie storica, né in fondo costringere il presente a perpetuare indefinitamente se stesso, ma accetta che la storia avanzi, purché lo faccia come espansione, e non negazione, dei valori che la tradizione ci consegna.

Né in questo conservatorismo, né più specificamente in Comte mancano aspetti di tortuosità a proposito dell'interpretazione della storia. Lo si vede, fra l'altro, quando Comte rievoca il tema cardine della sociabilità inserendolo nello svolgimento storico. La perfezione in questo campo sembra hegelianamente collocarsi in un futuro che dà tuttavia senso anche al passato: "Tutte le fasi della vita preparatoria [all'ordine positivo] devono concorrere all'istituzione dell'esistenza definitiva. Questa universale convergenza del passato verso l'avvenire risulta dal fatto che il problema umano in fondo è stato sempre lo stesso, è consistito ovunque nel costituire, fin dove è possibile, l'unità generale della nostra natura, individuale e collettiva" ${ }^{31}$. Le utopie moderne, che si sono poste il medesimo scopo di armonia universale del positivismo, hanno fallito secondo Comte perché non hanno saputo innestare la previsione del futuro sulla conoscenza e sul rispetto del passato. Hanno ignorato, in altri termini, i legami di continuità fra le generazioni: mentre l'Umanità religiosamente intesa, vale a dire il "Grande Essere" della terminologia comtiana, è "l'insieme degli esseri passati, futuri e presenti, che concorrono liberamente a preparare l'ordine universale" ${ }^{32}$.

\subsection{La religione dell'Umanità e il suo culto}

Ad apertura della "Teoria generale della religione, o teoria positiva dell'unità umana", compresa nel secondo tomo del Système de politique positive, Comte sintetizza con queste parole i propri intendimenti in termini di religione dell'Umanità: "Dapprima spontanea, poi ispirata, e in seguito rivelata, la religione diventa infine dimostrata. La sua costituzione normale deve soddisfare insieme il sentimento, l'immaginazione e il ragionamento, fonti rispettive dei suoi tre modi

\footnotetext{
31 31. A. Comte, Système, cit., t. I, pp. 16-17.

${ }^{32}$ Ivi, p. 30.
} 
preparatori. Inoltre essa abbraccerà direttamente l'attività, che né il feticismo, né il politeismo e tantomeno il monoteismo poterono mai consacrare abbastanza",33. La prima conseguenza di questa vasta definizione risiede nella necessità di una religione anche per la società positiva. Ogni aggregato umano ha del resto bisogno di una credenza comune che sottolinei e sacralizzi i legami unitari: la differenza della religione positiva, rispetto alle confessioni precedenti, risiede soprattutto nel fatto che la subordinazione dell'individualità all'ordine collettivo è ora frutto non di costrizione o di inganno, ma di convincimento fondato sull'osservazione. Come in biologia il corpo singolo dipende dall'ambiente, così in sociologia la religione positiva ci insegna la dipendenza dell'individuo dall'insieme.

La subordinazione all'ordine universale è secondo Comte il prodotto di due forze congiunte: la riflessione intellettuale e l'adesione morale (o, se vogliamo riprendere termini già più volte evocati, lo spirito e il cuore). Tutto l'apparato della religione positiva deve quindi risvegliare l'intelligenza e insieme il sentimento, unendo alla credenza l'amore. La ragione serve a costruire il dogma della sussistenza di un ordine universale immutabile, per quanto dinamico nelle sue variazioni interne dovute al progresso (che l'ultimo Comte definisce peraltro secondarie rispetto alla fissità primaria delle leggi naturali). Il sentimento rinforza il dogma soccorrendolo con l'affetto, il quale ha il compito, oltre che di mobilitare le energie morali in senso altruistico, di far credere che anche la parte dell'evoluzione umana che non è ancora possibile conoscere sperimentalmente si allineerà al progetto dell'ordine universale. Il riconoscimento di sacralità dovuto all'ente collettivo che si chiama Umanità richiede infine che le istanze unitarie diventino elemento di pratica quotidiana, ispirino l'azione dopo il pensiero e il sentimento.

Il culto dell'Umanità così proposto nelle sue grandi linee giunge nell' argomentazione di Comte al termine di un lungo processo storico, che ha fatto attraversare alle credenze umane le fasi imperfette del feticismo, del politeismo e del monoteismo. Come già a proposito della storia complessiva dell'umanità, l'interpretazione di Comte non rimane tuttavia fedele a un implicito schema lineare, che potrebbe far pensare a un progressivo avanzamento del genere umano verso le verità del culto positivo. Fra tutte le credenze passate, che pure si spiegano attraverso i loro intimi legami con l'ambiente storico, Comte sente più vicine a sé non le più recenti, ma la più arcaica: il feticismo. Incline come sappiamo a inserire elementi di circolarità nella sua argomentazione, il fondatore del positivismo apprezza infatti la spontaneità presente nelle più antiche forme di credenza nella sacralità dei fenomeni naturali. Il feticismo ha certo errato, quando ha esagerato nelle analogie fra uomini, animali, piante, esseri inanimati, ma ha avuto il pregio di attribuire "tutti i fenomeni materiali alle affezioni dirette fra esseri corrispondenti" $"$.

\footnotetext{
${ }^{33}$ Ivi, t. II, p. 7.

${ }^{34}$ Ivi, t. III, p. 70.
} 
Nell'apprezzamento per il feticismo, a confronto con le fasi successive della storia religiosa dell'umanità, Comte ribadisce implicitamente i suoi dubbi sui rischi di artificiosità e di orgoglio personalistico presenti nella ragione individuale. Poco soggettivo, più proiettato a immergersi amorevolmente nella grandezza dei fenomeni naturali oggettivi, il feticismo è nella visione comtiana estraneo alla superbia presente invece nel politeismo e nel monoteismo, portati a esaltare i propri dèi al di sopra della natura. Questa insistenza sui legami nascosti fra sentimentalismo originario e destinazione conclusiva del discorso religioso perseguito dal positivismo, corrisponde nel piano di Comte all'intenzione di mantenere aperta sul terreno della conoscenza e dell'azione la porta della semplicità, in linea del resto con l'ipotesi di avere come adepti privilegiati i proletari e le donne ${ }^{35}$. Nella ripresa di motivi "feticisti" si manifesta inoltre la volontà comtiana di costruire un credo religioso con una minima presenza di dogmi. Erede in ciò del deismo sei-settecentesco, il fondatore del positivismo cerca di evitare le assurdità presenti nelle dottrine religiose tradizionali, e di delineare un apparato di fede di impianto naturalistico, con la natura e l'umanità poste al centro della credenza comune. L'aspetto debole della sua predicazione, dal punto di vista della modernità, sta però nel fatto che, a differenza dei deisti, Comte non accetta di ridurre, insieme con i dogmi, anche le espressioni di culto. Egli vuole al contrario ribadire la necessità, anche per una religione che pure dovrebbe ricavare le sue verità dalla scienza, di un profilo basato su un esteso apparato di culto, corrispondente al bisogno di ritualità proprio di una società di massa.

Che la religione positiva sia soprattutto culto, ritualità, è più volte ribadito dal suo stesso fondatore. Nella fitta rete di corrispondenze fra la religione e il resto dell'impianto della filosofia positiva disegnate da Comte nel Système, il culto viene trattato prima degli altri elementi che compongono la religione dell'Umanità, vale a dire il dogma e il regime ${ }^{36}$. Questa precedenza non è soltanto argomentativa, ma riflette una gerarchia di intendimenti presente con chiarezza nell'ultimo Comte. Privilegiare nella religione positiva i riti rispetto alle idee, racchiuse nel dogma, e

\footnotetext{
${ }^{35}$ A proposito della persistenza di motivi già appartenuti al feticismo nell'impianto della religione positiva, interessanti sono le osservazioni avanzate da F.E. Manuel nei Profeti di Parigi, cit., in particolare alle pp. 340-341. Manuel vede in questa convinzione di Comte un tratto importante non solo della propensione per il culto esteriore, ma delle operazioni psicologiche complessive in cui l'autore del Système vede impegnata in prospettiva la predicazione positivista. Mentre Condorcet, commenta Manuel, tendeva a eliminare dall'orizzonte della conoscenza le immagini "primitive", composte di erronea elementarità, Comte le ripropone invece come atteggiamento psicologico ed educativo capace di diffondere l'amore universale. Ovviamente tutto ciò va interpretato alla luce del rovesciamento di gerarchia fra ragione e sentimento, che Condorcet non avrebbe certo accettato.

${ }^{36}$ Prigioniero delle sue solite argomentazioni ternarie, Comte sviluppa a proposito della religione positiva una serie di corrispondenze prodotte da un atteggiamento schematico, rivelatore comunque delle intenzioni strategiche del loro autore. All'articolazione fondamentale del positivismo fra amore, ordine e progresso corrispondono, nella religione positiva, i momenti del culto, del dogma e del regime, che a loro volta riflettono, della natura umana, le componenti del sentimento, dell'intelligenza e dell'attività. Una corrispondenza analoga può essere estesa all'educazione positiva, che è a sua volta affettiva, speculativa e attiva, o ai tempi della storia: rispettivamente il futuro, il passato e il presente. Il futuro non è dunque progresso, ma amore: il che ribadisce l'intenzione utopica del nostro filosofo.
} 
ai legami fra spiritualità e organizzazione della vita materiale insiti nella parola "regime", costituisce un'operazione moralizzatrice in sintonia con l'apprezzamento per il cuore prima che per lo spirito. E significa ancora una volta porsi il problema di una specie di evangelizzazione dei ceti popolari, intellettualmente votati alla semplicità, che riti suscitatori di emozioni estetiche sono in grado di unire all'ordine universale più di ogni sofisticato ragionamento.

Il culto trova la sua ragione complessiva d'esistenza nell' adorazione del Grande Essere (l'Umanità), rappresentando la "idealizzazione sintetica dell'esistenza che deve perfezionare" ${ }^{37}$. E a questo proposito che Comte elabora uno dei neologismi destinati a diventare nelle pagine dei critici una delle immagini più tipiche del suo pensiero, vale a dire sociolatrie, con cui indica l'adorazione dell'unità collettiva implicita in ogni richiamo alla religione dell'Umanità, che si regge sulla preminenza della specie rispetto all'individuo. Come si è già detto, Comte non si limita tuttavia a enucleare i caratteri di fondo del nuovo credo, ma prolunga la sua riflessione nella descrizione puntuale delle componenti del culto, comprese le più minute. Esse costituiscono il momento in cui la sua argomentazione più si avvicina a quella tradizionale degli utopisti, anche se qui non è possibile riproporla che nei suoi termini generali.

Anzitutto va sottolineato che, come esistono una morale privata e una sociale, rinforzate da un'educazione domestica e una pubblica, così il culto si delinea come privato e pubblico. Il culto privato ha una dimensione personale e una familiare, mentre il culto pubblico è determinato dal coinvolgimento simultaneo (estetico e sentimentale insieme) dell'intera comunità in una ritualità organizzata. Risultante di tutto ciò è "una progressione sociolatrica in cui ogni anima si lega successivamente alla Famiglia, alla Patria, all'Umanità, per dare regolarità alla coltivazione delle disposizioni che fanno sempre più sentire e comprendere il Grande Essere al fine di meglio servirlo" ${ }^{38}$. Il culto personale, che presuppone fra l'altro la recitazione di vere e proprie preghiere, consiste per l'uomo nell'adorazione della propria donna come emblema concreto dell'Umanità, per la donna nella celebrazione dei legami affettivi interni alla famiglia, per tutti nella sacralizzazione della solidarietà che deve reggere l'economia familiare. Il culto domestico collega il sentimento religioso individuale con le pratiche della credenza collettiva: memore della tradizione cattolica, Comte ritaglia a questo proposito uno spazio determinante per i sacramenti ${ }^{39}$. La ritualità domestica si prolunga infine in

\footnotetext{
${ }^{37}$ A. Comte, Système, t. V, p. 93.

${ }^{38}$ Ivi, p. 108.

${ }^{39}$ Comte prevede l'istituzione di nove sacramenti, il primo dei quali è la presentazione al sacerdote dell'Umanità, da parte dei genitori, dei figli appena nati. A quattordici anni avviene l'iniziazione, allorché i giovani passano dall'educazione familiare a quella sacerdotale; per i più dotati, a ventun'anni, può scattare l'ammissione a servire il Grande Essere. Solo per i maschi vale il quarto sacramento, la destinazione, con il quale ognuno, a ventott'anni, viene avviato dal sacerdote alla sua professione definitiva; alla stessa età i maschi possono contrarre matrimonio (l'età minima per sposarsi è invece di ventun'anni per le femmine). Comte sottolinea che, nell'ottica del culto positivo, il matrimonio è il più importante dei sacramenti. A quarantadue anni viene celebrata la maturità, vale a dire il definitivo inserimento nel Grande Essere. I tre ultimi sacramenti riguardano le fasi conclusive
} 
quella collettiva, per la quale Comte offre una serie di indicazioni anche troppo particolareggiate. Di queste vale la pena di ricordare l'incidenza, già citata, del culto dei morti, che ha il suo centro nella visita alle tombe; i processi di santificazione cui sono sottoposte nella memoria collettiva le grandi personalità, per lo più maschili, ma con qualche eccezione anche per le donne che più hanno meritato per la causa del positivismo; la necessità di costruire templi per il nuovo credo, le cui chiese saranno orientate verso Parigi, capitale della religione dell'Umanità (in ovvia analogia con quanto avviene per le moschee, orientate verso la Mecca); la concezione del rito come integrazione fra poesia e musica, con una funzione che va riconosciuta anche alla pittura e alla scultura, delegate a produrre monumenti all'Umanità.

All'interno del culto pubblico un ruolo essenziale è occupato dal progetto di riformare il calendario, che costituisce uno dei luoghi del Système de politique positive in cui è più evidente la filiazione dell'ultimo Comte dalle influenze della tradizione utopistica. Creare un nuovo calendario significa naturalmente porsi nell'ottica di una rifondazione del tempo. Questa era l'operazione dei repubblicani francesi della Grande Rivoluzione, che avevano voluto riavviare il computo del tempo considerando il 1792 l'inizio di una nuova era. E questa è l'operazione, almeno in superficie, di Comte, che un po' stranamente, date le sue preferenze per quella della Convenzione tra le varie fasi rivoluzionarie, sceglie come "anno zero" il 1789, una data dalle risonanze meno radicali rispetto al primo modello repubblicano della storia francese. Ma le analogie fra il calendario rivoluzionario e il calendario positivista si arrestano alla generica istanza della riformulazione del tempo: per il resto, le due costruzioni divergono profondamente. Se i repubblicani del 1792 si ripromettevano di scristianizzare la Francia, per i positivisti il loro calendario non è un imperioso atto di laicità che richieda una riformulazione del tempo su canoni esclusivamente naturalistici. Anche se finalità del genere non mancano del tutto a Comte, il suo intendimento sembra più quello di allargare il ritualismo proprio della tradizione cristiana fino a comprendervi figure care alla riflessione storica e filosofica positiva, piuttosto che di rovesciare quella tradizione. Tanto che l'elemento più caratteristico del calendario positivista risulterà alla fine essere quello della sostituzione, giorno per giorno, dei santi del culto cristiano con il ricordo di personalità che a giudizio dei positivisti hanno illustrato il progresso delle conoscenze e delle realizzazioni umane, in sintonia con il culto dei morti propugnato dal loro maestro. Curiosamente, ma non tanto, vista la posizione complessiva di Comte, fra queste personalità riappare anche più di un santo della cristianità, accanto (come vedremo) a filosofi, scienziati, scrittori, uomini politici, legislatori, condottieri.

dell'esistenza individuale: a sessantatré anni avviene il ritiro dalla vita attiva; la morte viene ricordata con un rito di trasformazione; infine, sette anni dopo la morte i defunti particolarmente meritevoli possono essere dichiarati degni di venerazione da parte della comunità, il che comporta un rito di incorporazione nella schiera di coloro che meglio hanno servito il Grande Essere. 
Va inoltre considerato che, procedendo come al solito per accumulazione di dati e suggestioni, che spesso inducono nel lettore l'impressione di trovarsi all'interno di un labirinto intellettuale, Comte produce a partire dal 1849 più versioni del calendario e dei suoi criteri ispiratori. Le indicazioni comprese nel quarto tomo del Système, che riassumerò fra breve, sono in parte diverse dal disegno di calendario che gira negli stessi anni fra i circoli positivisti, e che appare in appendice allo stesso volume. Elemento comune a tutte le versioni è tuttavia la proposta di Comte di costruire un'armonia fra il ciclo solare e quello lunare, da cui deriverebbe un anno di tredici mesi di quattro settimane ciascuno, più un giorno supplementare che diverrebbe doppio negli anni bisestili.

Nella versione analitica proposta dal Système, la consacrazione dei mesi deve celebrare le componenti della società positiva e ricostruire il progresso sociale e storico delle credenze umane fino al raggiungimento della fede positiva. Dopo aver dedicato il primo mese all'adorazione sintetica dell'Umanità, il calendario prevede quindi cinque mesi in cui si celebrano i rapporti privati sui quali poggia il legame pubblico: si tratta del matrimonio, della paternità, della filialità, della fraternità, della domesticità. Tre mesi sono poi riservati alla celebrazione dinamica delle fasi preparatorie alla religione positiva (il feticismo, il politeismo e il monoteismo), mentre i quattro conclusivi idealizzano la sociocrazia attraverso la sacralizzazione dei suoi elementi: la donna, il sacerdozio, il patriziato e il proletariato. Quanto ai giorni della settimana, Comte ipotizza che possano essere intitolati alle sette personalità che più hanno contribuito alla transizione occidentale dalla teocrazia alla sociocrazia (sono Omero, Aristotele, Cesare, San Paolo, Carlomagno, Dante, Cartesio), oppure alle sette scienze fondamentali del positivismo.

È significativo che qui, come in altre parti del Système, Comte accenni alla morale come settima e più elevata scienza, dopo le sei già codificate nel Cours. Il che ribadisce l'impegno soprattutto moralizzatore dell'ultimo Comte: se ricordiamo infatti che nel suo sistema ogni scienza si concatena necessariamente con quelle precedenti, mentre prima era la sociologia a determinare gli esiti conclusivi delle scienze della natura, ora è la morale a sovraordinare il legame fra ogni conoscenza, naturale o sociale che sia. Lo si deduce dall'immagine sintetica che lo stesso Comte ci offre a un certo punto della religione positiva, descritta come "la concezione relativa dell'ordine universale, necessariamente scomposto in sette categorie, sovrapposte in modo che ognuna modifica la precedente e domina la seguente". Le categorie ora sono dunque sette, e non più sei: alle scienze speciali si è aggiunta definitivamente la morale. "Questo seguito di dominazioni modificate sfocia nella rappresentazione dell'uomo come il riassunto normale e il regolatore spontaneo dell'ambiente sociale, vitale e materiale, sotto cui egli si sviluppa. Ma la sua azione individuale, destinata a perfezionare la fatalità con la volontà, diviene efficace e degna solo ponendosi liberamente al servizio continuo del Grande Essere, di cui egli costituisce l'elemento indivisibile e il prodotto necessario" ${ }^{40}$.

${ }^{40}$ A. Comte, Système, cit., t. IV, p. 185. 
Non vale la pena di seguire Comte in tutte le più minute articolazioni del calendario e della ritualità ad esso connessa, che egli affronta con una prolissità ancora superiore al consueto ed evidenziando più di un tratto di maniacalità ${ }^{41}$. Quel

${ }^{41}$ È tuttavia opportuno ricordare la struttura assunta dalla versione circolante del calendario positivista, nella quale la consacrazione dei mesi segue sistematicamente l'evoluzione della storia e del pensiero dell'umanità dalle origini all'avvento del positivismo. Analogamente a un calendario cristiano con i suoi santi, ogni giorno dell'anno è caratterizzato dalla dedica a una o più personalità storiche o mitiche. Il primo mese è dedicato a Mosè, ossia alla "teocrazia iniziale": i singoli giorni delle settimane ospitano il ricordo dei mitici legislatori del mondo occidentale (come Licurgo e Romolo), dei fondatori delle religioni del Medio Oriente e dell'India (fra cui Zoroastro e Budda), dei predicatori dell'Estremo Oriente (con in testa Confucio), dei profeti della tradizione giudaica e islamica, da Abramo a Maometto. Singolare risulta l'esclusione di Gesù Cristo, decisa da Comte e dai suoi fedeli più stretti dopo lunghe esitazioni, e senza spiegazioni convincenti sulle sue ragioni: "strappo" nei confronti del cristianesimo, o implicito rispetto per una sacralità superiore che non si vuole abbassare al livello delle altre citate nel calendario? Il secondo mese è consacrato a Omero, rappresentante della "poesia antica": nelle indicazioni dei personaggi storici da celebrare in questo mese la concezione di poesia, che riserva considerazione per tutti gli autori dell'epica, della drammaturgia e della favolistica antiche, si estende anche alle arti figurative, in particolare alla scultura dell'antica Grecia. Il terzo mese è dedicato alla filosofia antica, sintetizzata con il nome di Aristotele: nei giorni e nelle settimane che la compongono trovano comunque posto tutti i maggiori filosofi della classicità greca e latina (questa volta anche Platone), più qualche santo della cristianità come Giovanni Evangelista. Il quarto mese è quello in cui si ricorda, specialmente nel nome di Archimede, la scienza antica, che Comte celebra facendo riferimento ai più noti matematici, medici, geografi, architetti dell'antichità: degno di attenzione è l'inserimento, fra gli scienziati quindi, e non fra i letterati, di Plutarco. Con Cesare, cui è consacrato il quinto mese, si passa al ricordo della "civilizzazione militare": i singoli giorni sono dedicati ai grandi condottieri dell'antichità greca e latina, con l'inserimento tuttavia anche di Annibale. Il sesto mese celebra il cattolicesimo nel nome di San Paolo: quasi tutti i singoli giorni conducono al ricordo di santi della chiesa cattolica vissuti nei primi secoli del cristianesimo, ma alla fine del mese viene trovato uno spazio per personalità più recenti, da Ignazio di Loyola al predicatore quacchero William Penn, fino all'amato Bossuet. Il settimo mese, dedicato alla civiltà feudale e alla sua personalità più rappresentativa, Carlomagno, insiste soprattutto sulla celebrazione di imperatori e santi "combattenti" (anche in senso morale) della cristianità medievale. Con Carlo Martello e San Luigi, oppure con alcuni sovrani d'Inghilterra, troviamo ricordati così anche Giovanna d'Arco, San Francesco e Thomas Beckett, con una curiosa incursione verso Oriente con la citazione del Saladino. L'ottavo mese è consacrato alla "epopea moderna", con la figura di Dante in rilievo: la dedica dei giorni si muove dall'epica tardomedievale all'arte rinascimentale, per arrivare ad autori moderni cari a Comte come Chateaubriand, Manzoni e Scott. Nel nono mese si celebra l'industria moderna, che ha il suo rappresentante di spicco in Gutenberg: i personaggi ricordati vanno dai navigatori come Marco Polo o Cook agli innovatori nel campo dell'artigianato e della tecnologia, da Cellini fino a Watt e ai Montgolfier. Segue il mese (il decimo dell'anno) consacrato al dramma moderno nel nome di Shakespeare: in esso si riveriscono gli autori della drammaturgia europea moderna ma anche i grandi musicisti, fra cui Beethoven, Mozart, Bellini, Rossini. L'undicesimo mese onora la filosofia moderna, con a capo Cartesio: partendo dai grandi filosofi cristiani del medioevo, in particolare da San Tommaso, Comte giunge fino ai suoi giorni, ricordando tutti i grandi filosofi fino a Hegel. Una sola è l'assenza, ma rumorosa: Rousseau. Tocca poi (dodicesimo mese) alla politica moderna, di cui è emblema Federico II: durante il mese sono rammentati sovrani noti per gli atteggiamenti più o meno illuminati, e altri uomini politici e di governo, fra cui Turgot, Franklin, Washington, Jefferson, Bolívar. Appare pure qualche nome di scrittore impegnato anche in missioni politiche, fra cui Comenio e Guicciardini: e si staglia così, dopo Rousseau, un'altra rilevante assenza da un calendario che rammenta quasi tutto il celebrabile, e cioè il 
che è importante ribadire è che si tratta di un episodio, particolarmente insistito, dell'espansione che il culto deve avere nel celebrare i legami sociali. Il dogma, parte più intellettiva della religione dell'Umanità, ha in questa strategia il compito sussidiario di completare il culto e di preparare il regime. La natura generale del dogma positivo è data dal programma di armonizzazione fra teoria e pratica. $\mathrm{Ne}$ sono caratteri universali la necessità di combinare i sentimenti con le immagini e con i simboli per rinforzare l'intelligenza, il tutto volto a ribadire l'immutabilità delle leggi naturali fondamentali e la loro variabilità secondaria come perfezionamento in senso evolutivo. Nell'ambito della trattazione definitiva del dogma positivo, affidata all'ultimo tomo del Système, Comte ripercorre così con piglio spiritualizzante l'itinerario che dalle singole scienze conduce alla morale. Fra i concetti ribaditi a questo proposito con la consueta radicalità, spicca ora l'obiettivo di incorporare la scienza profana nella scienza sacra. Lo scopo è al solito quello di evitare gli abusi dell'intellettualismo: meglio attrezzata in proposito è la donna, più in grado dell'uomo di coordinare la vita cerebrale con l'esistenza corporale, la teoria con la pratica, l'individualità con la comunità. L'utopia della Vergine Madre, nata da considerazioni aventi a che fare con la sessualità, si riaccampa ora come limite ideale destinato a riassumere il perfezionamento umano in tutte le sue combinazioni.

Approdo conclusivo di tutto l'impianto della religione positiva, che la riconduce ai suoi scopi terreni, è la costruzione del regime positivo. Esso diventa, al termine dell'argomentazione comtiana, qualcosa di più della società positiva tratteggiata nel Cours e nei primi volumi del Système: non è solo un'immagine di società perfetta prodotta dalla storia, ma un vero e proprio organismo politico-religioso, il cui senso più profondo sta appunto nella corrispondenza ai voleri del "Grande Essere". La necessità del regime proviene dall'esistenza di bisogni materiali, che presuppongono un apparato di attività produttive, le quali vanno tuttavia ricondotte all'unità di intenti e di realizzazioni che permea di sé tutto il positivismo comtiano. Organizzare la produzione verso i bisogni collettivi spetta al patriziato: ma la sorveglianza sulla corrispondenza fra la realtà e gli obiettivi spetta al potere spirituale.

Prima delle sue pagine conclusive sulla religione dell'Umanità, quelle comprese appunto nell'ultimo tomo del Système, Comte ha più volte evocato l'importanza del sacerdozio positivista, sia come luogo di elaborazione filosofica, sia come organo di sorveglianza religiosa. $\mathrm{E}$ ne ha già descritto alcuni caratteri di fondo: il numero necessariamente piccolo dei suoi membri, perché in pochi si trovano le doti intellettuali e morali adatte a un compito così speciale; la loro disciplina e organizzazione all'interno di un vero e proprio clero, con tanto di cariche e di capo supremo (il Grande Prete dell'Umanità, all'inizio, ovviamente, lo stesso Comte); l'opportunità di sciogliere il clero così formato da qualsiasi impegno nell'amministrazione, per non corromperlo o comunque distoglierlo dalla sua

nome di Machiavelli. L'ultimo mese dell'anno è dedicato alla scienza moderna, sotto il nome di Bichat: tutti gli scienziati più importanti, da Copernico in poi, sono qui ricordati. 
missione spirituale con la cura degli affari materiali; il suo triplice impegno come elaboratore della verità oggettiva, suo diffusore nel campo sociale e controllore morale degli atti del potere materiale. Di tutto questo l'ultimo Comte ama evocare soprattutto il profilo morale del sacerdote e della sua presenza nella società positiva. All'interno della compagine clericale, questa mobilitazione morale significa rinuncia all'uso individualistico della ragione: "La religione positiva supera le disposizioni critiche che il suo preambolo scientifico ha sviluppato e che l'iniziazione teorica riprodurrebbe senza una sorveglianza costante. Essa coltiva la dimostrazione al fine di istituire non la discussione sterile o dispersiva, ma la sottomissione attiva e volontaria che deve nobilitare anche le fatalità più grossolane, collegandole al perfezionamento morale. Non è una fede dimostrata, ma una fede sempre dimostrabile, che conviene alla maturità della ragione umana, destinata a sviluppare le conseguenze senza deliberare sui princìpi" ${ }^{\prime 2}$. Comte continua a volersi lasciare aperta la strada del relativismo, proprio degli intendimenti conoscitivi del positivismo delle origini: ma è certo che la preminenza è ormai dedicata all'imperio del capo religioso (come in ogni confessione tradizionale), che richiede obbedienza anzitutto ai suoi collaboratori.

$\mathrm{Ma}$ ancor più importante per le idee politiche è quanto accade all'altro lato dell'argomentazione religiosa dell'ultimo Comte, quella rivolta non all'interno della chiesa, ma all'esterno, alla compagine sociale nella sua globalità. In quest'ambito la religione positiva è soprattutto ammaestramento morale, per cui la sintesi fra culto, dogma e regime diventa soprattutto un discorso educativo. "L'educazione positiva istituisce l'unità reale, insegnandoci a vivere per il prossimo. Destinata ad adattarci al servizio continuo del Grande Essere, resta soprattutto morale, anche quando diventa intellettuale. Fondata sull'esistenza naturale delle tendenze simpatetiche, subordina a queste ultime gli istinti personali" ${ }^{43}$. Le esemplificazioni che Comte produce di questa influenza morale sono parecchie, e talune preoccupanti per uno spirito laico, come poteva essere all'epoca quello di un Littré. Infatti non si tratta soltanto dell'obiettivo comprensibile di ricondurre con la mobilitazione morale il potere politico ai suoi fini di soddisfazione dell'interesse generale. Né il compito della religione positiva si limita, per citare un caso specifico avanzato da Comte, a estendere l'influenza morale del sacerdozio a una rigorosa programmazione del numero dei membri delle varie famiglie, per offrire stabilità alla società positiva. Si tratta di uno strumento di pianificazione demografica forse discutibile, anch'esso proprio fra l'altro di consolidate ispirazioni provenienti dalla letteratura utopistica, ma che nasconde comunque un nodo, quello dell'entità della riproduzione, non rinviabile a sole considerazioni e pulsioni private, ma oggettivamente destinato a diventare argomento di politiche pubbliche. Comte va più in là di queste comprensibili integrazioni fra morale e politica: egli si spinge a disegnare una funzione del clero, alleato con le donne, nell'estirpare i vizi, dall'abuso dell'alcool alle pratiche

\footnotetext{
${ }^{42}$ A. Comte, Système, cit., t. IV, p. 267.

${ }^{43}$ Ivi, p. 260.
} 
sessuali non disciplinate. E per rendere efficace il controllo evoca un principio che sarà tipico dei regimi totalitari: quello della necessità di una crescente pubblicità degli atti privati, affinché possano essere di fatto sottoposti al giudizio costante del sacerdote $^{44}$. Intendiamoci: con queste notazioni Comte non si trasforma in un ayatollah trapiantato a Parigi, ma è casomai l'erede un po' infatuato della commistione fra credo e morale propria di ogni tradizione religiosa, e che nella storia europea aveva avuto varie incarnazioni, dal cattolicesimo tridentino al puritanesimo calvinista. Resta tuttavia il fatto che l'impianto morale e religioso del suo pensiero, che occupa pesantemente una buona metà della sua ultima opera e condiziona l'altra metà, è destinato a lasciare un'impronta difficilmente separabile dal resto dell'argomentazione positivista, quella di carattere più decisamente scientista.

Ritornando alle distinzioni littréane da cui siamo partiti, possiamo concludere affermativamente in quanto all'esistenza di due Comte, forse non proprio disgiungibili in termini cronologici, perché più intimamente uniti nelle varie fasi della loro formazione, ma comunque distinguibili fra un inizio più razionalistico e una fine più marcatamente spiritualistica. Da qui l'ambiguità del messaggio comtiano per i posteri, che in effetti potranno ritrovare nelle sue pagine ispirazione per idee politiche anche molto differenti fra loro. Più che un dualismo troviamo anzi nel lascito comtiano una molteplicità di umori: perché nell'unione fra Cours e Système abbiamo un modello scientista che diventa un sistema di sapere chiuso; un'interpretazione della storia che si trasforma in un rigido ingabbiamento previsionale del futuro; una dottrina del progresso che si rovescia in una compiuta e ordinata programmazione morale; un avvicinamento al motivo del perfezionamento umano che scivola in una concezione religiosa arazionale; e ancora una visione gerarchica della politica, una comunicazione che dal potere deve scendere verso le masse, ma che vorrebbe coesistere con le aspirazioni che dal proletariato salgono a reclamare considerazione presso la politica più elevata. Troppe cose, in fondo, per non dare l'impressione di un sistema all'apparenza coeso, ma portato anche a evidenziare possibili lacerazioni interne e comunque poco duttile per confrontarsi col mondo reale senza dimostrare il bisogno di correzioni e adattamenti. E soprattutto senza ispirare nel lettore l'impressione che, più che risolto dai tanti e potenti richiami all'armonia universale, rimanga eluso un problema rilevante per la stessa cultura positivista, quello della libertà della ragione e dei suoi eventuali confini.

\footnotetext{
${ }^{44}$ Cfr. ivi, p. 312.
} 



\section{Dopo Comte: positivismo moderato e positivismo radicale}

\subsection{Littré e il positivismo della Terza Repubblica}

Capita abbastanza spesso, nelle opere generali, di trovare la definizione di "età del positivismo", impiegata per indicare uno specifico periodo dell'Ottocento (in particolare gli anni settanta e ottanta), qualche volta l'intera seconda metà del secolo $\mathrm{XIX}^{45}$. Come tutte le definizioni che cercano di offrire un'immagine complessiva di un'epoca, anche questa dice troppo, e troppo poco. Dice troppo se allude a un'egemonia culturale della filosofia positivista, la cui influenza rimane nei fatti abbastanza lontana dal permeare di sé un'intera epoca. Dice troppo poco se pretende di rinviare implicitamente, sotto l'etichetta di "positivista", a un impianto di pensiero unitario e compatto, che nella realtà si presenta invece articolato in movimenti e correnti sensibilmente diversi fra loro.

Se consideriamo il positivismo dal punto di vista del costume culturale, possiamo senz'altro misurare un suo progressivo inserimento nelle attività intellettuali e nei gusti del pubblico dopo la morte di Comte ${ }^{46}$. Per rimanere nell'area che qui ci interessa maggiormente, quella francese, possiamo anzitutto rinviare genericamente all'atmosfera di celebrazione della scienza e della tecnica che traspare nell'esperienza economica, politica e civile del Secondo Impero e ancor più della Terza Repubblica. A conferma di ciò dobbiamo ricordare

\footnotetext{
${ }^{45}$ Fra i molti esempi possibili, ricordo quello di Robert Mandrou, che intitola "La Francia positivista" il capitolo sulla seconda metà dell'Ottocento della Storia della civiltà francese scritta insieme con Georges Duby (Milano, Mondadori, 1980). Come è frequente nell'uso di tale titolazione, l'appellativo positivista rimanda a una generale atmosfera di tipo scientista, descritta come il sottofondo culturale più idoneo a spiegare fenomeni come la definitiva rivoluzione industriale.

${ }^{46}$ Per quanto riguarda la formazione e la diffusione della filosofia positiva rimando, oltre al già ricordato lavoro di Simon, a L. Kolakowski, La filosofia del Positivismo, Roma-Bari, Laterza, 1974; C.G.A. Bryant, Positivism in Social Theory and Research, Basingstoke and London, Macmillan, 1985; S. Poggi, Introduzione al positivismo, Roma-Bari, Laterza, 1987; all'opera collettiva Scienza e filosofia nella cultura positivistica, a cura di A. Santucci, Milano, Feltrinelli, 1982; al numero speciale di "Romantisme" (1978, 21-22) dedicato ai Positivismes.
} 
accadimenti culturali legati in forme più o meno continue al positivismo. Si pensi alla nascita di due riviste importanti generate direttamente da due delle anime del movimento, la "Philosophie positive" di Littré e la "Revue occidentale" di Laffitte, nonché di altre dipendenti in modo indiretto dal programma positivista, prima fra tutte la "Revue d'anthropologie" di Broca. A queste iniziative di gruppo si aggiunge la notorietà personale conseguita dai due eredi più famosi del pensiero di Comte: appunto Littré, che dopo aver raggiunto la fama come autore del Dictionnaire de la langue française entra prima all'Accademia di Francia e poi al Senato; e Pierre Laffitte, che tiene a cavallo del 1870 una serie di frequentatissime conferenze di divulgazione del pensiero comtiano, per assumere un ventennio dopo, al Collège de France, la prima cattedra di storia della scienza istituita in Francia. Si aggiunga ancora il ruolo rilevante, anche sul piano civile, assunto da scienziati di formazione positivista, come il medico e biologo Charles Robin (chiamato nel 1866, primo caso di positivista "militante", a far parte dell'Accademia delle Scienze), il fisiologo Claude Bernard, il chimico Marcellin Berthelot, tutte figure di primo piano del pensiero scientifico dell'epoca. Questi innegabili successi non significano però un imporsi altrettanto deciso del positivismo come dottrina filosofica. Se il suo ruolo come strumento di coordinazione fra le scienze speciali è generalmente riconosciuto, non avviene altrettanto per l'aspirazione comtiana di fare della filosofia positiva il riferimento di ogni spiegazione e azione umana, per il passato, il presente e il futuro: di farne, insomma, una vera e propria ideologia. Mentre le classi colte della seconda metà dell'Ottocento tendono a uniformarsi a un laicismo erede più del vecchio eclettismo che della filosofia di Comte (che del resto, al suo termine, tanto laica non poteva più apparire), le classi popolari trovano speranze per il loro riscatto nella continuità con le tradizionali dottrine socialiste o nell'incontro con la nuova cultura marxista, che Lafargue e il primo Sorel si danno da fare per divulgare in Francia. Per non parlare dell'influenza, pur meno intensa che in passato, mantenuta dal cattolicesimo su tutte le classi sociali.

Se la definizione di "età del positivismo" rimane dunque alla superficie dei fenomeni storici che evoca, quello che maggiormente ci interessa in questa sede è tuttavia il fatto che la locuzione dica troppo poco sulle articolazioni interne al movimento cui allude. Fra il 1860 e il 1890, che possiamo indicare come il periodo più fecondo della cultura positivista, assistiamo infatti ad almeno sue quattro diverse declinazioni. C'è anzitutto un positivismo per così dire "istituzionale", che penetra negli ambienti direttivi della Terza Repubblica e che ha in Littré il suo maggior esponente. È una corrente che per il suo crescente realismo e moderatismo rimane ai margini dell'intenzione di intrecciare scienza e utopia. Anch'essa mantiene comunque qualche analogia con le speranze di rifondazione del mondo formulate da Comte. C'è in secondo luogo un positivismo "ortodosso", intenzionato a recepire l'insieme del messaggio comtiano, a unire cioè all'esaltazione della scienza un sentimento altrettanto vivo di celebrazione religiosa 
dell'Umanità: ed è, come vedremo presto, soprattutto la funzione esercitata da Laffitte e dagli altri aderenti alla Société Positiviste. Al di fuori dei canali diretti di influenza comtiana, si sviluppa poi un terzo modello di positivismo, praticato soprattutto dagli scienziati di professione, che ricavano dallo scientismo comtiano non tanto una nuova metafisica, quanto al contrario una spinta per ridisegnare un ruolo significativo della scienza per la vita concreta degli uomini, al di fuori di troppe preoccupazioni filosofiche ${ }^{47}$. E c'è ancora il positivismo dei compagni di strada, degli intellettuali vicini alla filosofia comtiana per brevi fasi della loro vita oppure impegnati a trattenere di quella filosofia solo quanto serve al loro specifico operare: il positivismo di Renan, di Taine, di Zola.

Questa campionatura, per quanto schematica, autorizza insomma a parlare più di "positivismi" al plurale che di positivismo al singolare. Accanto a un nucleo di pensiero comune (che ha per componenti la conclamata necessità di costruire una visione scientifica dell'uomo, la destinazione morale da contemplare in ogni progetto conoscitivo, l'interpretazione della società e della politica in una prospettiva non solo razionalistica, ma anche storica, la definizione di un ruolo sociale per il sapiente) si sviluppano infatti convincimenti e strategie di comunicazione che consentono di parlare di una pluralità di tendenze culturali.

La prima delle correnti qui ricordate è dunque quella di Littré e dei collaboratori più assidui alla sua "Philosophie positive": il condirettore Wyrouboff, Stupuy, Robin, Fontpertuis. È l'ala del movimento più lontana dall'utopismo comtiano, ma la completezza del quadro ci impone di soffermarci ancora un momento sugli scarti e le analogie che essa rivela nei confronti dell'originario impianto positivista. Si è già fatto cenno, nel capitolo precedente, alle revisioni cui Littré sottopone la dottrina ereditata da Comte, incentrate soprattutto sulla valorizzazione del progresso scientifico e sulla definizione della filosofia positiva come strumento dinamico di unificazione delle scienze particolari, non come sistema chiuso ${ }^{48}$. La

\footnotetext{
${ }^{47}$ Come emblema di questo positivismo degli scienziati a bassa intensità filosofica, mi riferirò in questo capitolo soprattutto al caso di Berthelot. Secondo Simon (Il positivismo europeo, cit., in particolare alle pp. 127-129) la tentazione di separare filosofia e scienza è abbastanza diffusa già nei primi anni di consolidamento della cultura positivista, anche fra personaggi ritenuti a torto o a ragione vicini al movimento di pensiero fondato da Comte. Due esempi rappresentativi sono in proposito quelli di Cournot e di Bernard. Augustin Cournot, filosofo, scrive con Comte ancora vivo, e precisamente nel 1851, un Essai sur les fondements de la connaissance et sur les caractères de la critique philosophique: un'opera in cui riecheggiano espressioni come "scienza positiva" o "storia positiva", ma in cui alla fine si auspica il mantenimento di sfere distinte per la scienza e per la filosofia. Claude Bernard, scienziato, sembra aderire fino al 1865 al programma positivista di centralità della scienza nell'esperienza intellettuale, ma in seguito abbandona esplicitamente le costruzioni filosofiche più tipiche del comtismo. Giudica la legge dei tre stadi una nuova forma di metafisica e la religione dell'Umanità un cumulo di assurdità, né più né meno delle religioni che l'hanno preceduta.

${ }^{48} \mathrm{Su}$ Littré rinvio, oltre al mio studio già citato, alle monografie di A. Aquarone, The Life and Works of Emile Littré, Leyden, Sythoff, 1958; di A. Rey, Littré. L'humaniste et les mots, Paris, Gallimard, 1970; di J. Hamburger, Monsieur Littré, Paris, Flammarion, 1988; e soprattutto agli atti del Colloque Littré pubblicati nella "Revue de Synthèse", nn. 106-108, Paris, Albin Michel, 1982.
} 
complessità delle cose da conoscere (enorme quando si passa dalle scienze della natura alle scienze dell'uomo) comporta una grande prudenza nel ricavare generalizzazioni dai dati empirici. Essendo sia la ricerca del vero, sia la costruzione del mondo positivo un processo in continua evoluzione, nessuno può arrogarsi il potere di esaurire in sé ogni aspirazione alla libertà di conoscere e alla libertà di agire. La libertà individuale è peraltro un tema affrontato da Littré e dai suoi in un modo non privo di incertezze, del resto inevitabili per una cultura che crede nella possibilità per la scienza di raggiungere stabili verità oggettive, e le affida il compito di allargare i propri ambiti dalla natura all'uomo e alla società (quindi al regno non solo delle conseguenze della storia e dell'ambiente, ma anche della volontà, del desiderio e dell'opinione). Da un lato i collaboratori della "Philosophie positive" intendono togliere al positivismo la carica dogmatica conferitagli da Comte e confermata dal determinismo che spesso sorregge le scienze umane nascenti. Ma dall'altro non possono esimersi dal porre il problema di quanto sia giusto obiettare, in nome dell'autonomia personale, di fronte alle verità oggettive suffragate dalla ricerca scientifica. Non risolto, il dissidio potenziale fra libertà e determinazione è tuttavia messo in ombra dalla convinzione che, nell'ambito delle discipline potenzialmente più dirompenti nei confronti del tema della libertà di opinione (biologia e sociologia, secondo la classificazione positivista), le certezze ricavabili dai materiali conosciuti sono poca cosa rispetto all'ampiezza del conoscibile. Accanto alla fede nell'oggettività della scienza possono così coesistere propensioni altrettanto forti verso la libertà di pensiero e di stampa, la libertà di insegnamento o la tolleranza religiosa, valori che contrassegnano l'esperienza littréana in misura sconosciuta rispetto a qualsiasi altro esponente del positivismo francese ${ }^{49}$.

Un discorso ancora più dialettico vale per la storia, che è per Littré (come per Comte), la maggior fornitrice di materiali di studio per la sociologia. La sensibilità e la finezza con cui Littré affronta in tutta la sua vita di studioso questioni storiche come quelle riguardanti l'origine e l'evoluzione delle parole, oppure quelle legate allo sviluppo della scienza medica, dimostrano la sua convinzione della

\footnotetext{
${ }^{49}$ Sul tema dei rapporti fra libertà e verità scientifica tornano parecchi interventi sulla rivista fondata da Littré. Per offrirne un esempio, ricordo l'articolo dello stesso Littré sulla Condition essentielle qui sépare la sociologie de la biologie (nella raccolta definitiva della rivista appare nel vol. II, 1868), articolo volto a riconoscere una maggiore sfera di autonomia individuale nella conoscenza e nella pratica delle leggi sociali rispetto all'inevitabile determinismo di quelle naturali. Su linee analoghe si muovono gli interventi di Georges Wyrouboff, come quello intitolato Certain et probable, absolu et relatif, apparso nella prima serie della "Philosophie positive", del 1867. Massiccia è l'attività della rivista nel promuovere un riordino dell'istruzione pubblica basato sulla libertà di insegnamento e insieme sul raccomandato accoglimento della filosofia positiva fra i contenuti educativi. Segnalo in proposito, fra gli altri, l'intervento di Wyrouboff, Enseignement libre (nel vol. II, 1868); quelli di Littré, Projet de réorganisation de l'instruction publique en France (vol. VII, 1871) e Liberté de l'enseignement supérieur (vol. XIV, 1875); la serie di articoli dedicata ai Rapports de l'éducation avec l'instruction da Robin fra il 1876 e il ' 77 , raccolti poi in volume sotto il titolo L'instruction et l'éducation.
} 
complessità oggettiva del divenire storico e della lentezza dei suoi ritmi effettivi rispetto alle attese della ragione. La legge dei tre stadi non viene rigettata, perché resta comunque la base per una lettura progressiva della storia, ma va reinterpretata come uno schema essenziale che non può pretendere di rappresentare la pienezza dello sviluppo storico. Le "leggi" della sociologia, sia quando vengono applicate nelle ricerche riguardanti il passato, sia quando sono usate come fonte di previsione per il futuro, sono fra le più soggette al bisogno continuo di nuove e non precostituite indagini. Un discorso analogo vale per la politica. La società positiva è per Littré al massimo un'ipotesi regolativa, un ideale con cui confrontare l'impegno politico e morale dei positivisti, non certo l'incarnazione di un'attesa di perfezione assoluta. Se certe linee indicate da Comte (divisione fra potere spirituale e potere temporale, solidarietà fra le classi, collaborazione tra famiglie e istituzioni) rimangono valide anche per Littré come fonti generali di ispirazione per la politica positiva, quest'ultima non deve però orientarsi rigidamente verso i suoi princìpi astratti, ma accettare volentieri tutto ciò che nella politica del tempo consente di coordinare libertà, progresso e conservazione dell'ordine: il suffragio universale, il parlamento, le istituzioni repubblicane ${ }^{50}$.

Il positivismo del gruppo di Littré (che non per nulla si presenta come un gruppo aperto, tenuto insieme da interessi di ricerca e non dalla fede negli stessi dogmi) espelle così esplicitamente dal proprio orizzonte le speranze utopiche di rapida e definitiva rifondazione del mondo. In un passo del 1876 questo atteggiamento risalta con particolare evidenza: "La filosofia positiva [...] da parecchi anni raccomanda a ogni istante di prendere in considerazione la storia di un popolo e dei suoi antecedenti, la lentezza delle modificazioni dello spirito pubblico, il pericolo delle concezioni metafisiche e assolute nelle questioni sociali, la natura relativa di tali questioni sempre subordinate ai tempi e ai luoghi, la necessità di dedicarsi a ciò che comporta una soluzione vicina: un insieme di dottrine, in una parola, che mostrano come sia più sicuro e abile affidarsi al movimento spontaneo di una società dirigendolo, anziché seguire progetti razionali, per quanto questi possano sembrare plausibili" ${ }^{\prime \prime}$.

L'atteggiamento laico, pragmatico, tendenzialmente liberale e sostanzialmente democratico dei littréisti non giunge tuttavia al rigetto esplicito della religione dell'Umanità. Se del pensiero religioso di Comte viene rifiutato il culto (per

\footnotetext{
${ }^{50}$ Una buona rappresentazione delle idee politiche di Littré nel periodo di formazione della Terza Repubblica è costituita dal suo volume De l'établissement de la Troisième République, Paris, Bureaux de la philosophie positive, 1880, che raccoglie scritti pubblicati nella "Philosophie positive" e in altre riviste.

${ }^{51}$ E. Littré, Éducation politique, "Philosophie positive", nov.-dic. 1876, poi in De l'établissement, cit., p. 388. Littré aveva già rifiutato esplicitamente l'operazione utopistica di descrivere la società positiva come un mondo chiuso e definitivo nella seconda edizione delle Paroles de philosophie positive, apparsa nel 1863. La stessa posizione è ribadita da Wyrouboff sulla "Philosophie positive" nel 1881, come apprezzamento del metodo gradualista della Terza Repubblica, e segnatamente di Gambetta, nel favorire il progresso sociale e culturale.
} 
esempio, il calendario positivista viene degradato a mera occasione di memoria storica, senza alcuna implicazione di sacralità), resta però in piedi l'idea che il mantenimento di una credenza collettiva sia utile al perseguimento di una società insieme progredita e ordinata. Emerge a questo proposito una delle maggiori continuità fra Littré e il suo maestro, e cioè l'interpretazione del positivismo anche come una grande opera di mobilitazione etica. Littré non è ovviamente per l'annullamento dell'individuo nella comunità, ma per la ricerca di un giusto equilibrio fra autonomia del singolo e sua collaborazione, liberamente scelta, alle finalità di miglioramento progressivo della specie. La morale diventa in quest'ambito uno strumento per allontanarsi dalle tensioni affiorate nel pensiero di Littré a proposito della libertà. L'adeguamento alle verità scientifiche, in tutti i campi in cui queste si presentino con sufficiente attendibilità, viene affidato non a un atto di imperio o di costrizione da parte del potere (sia esso temporale o spirituale), ma a un processo di riflessione individuale e collettiva che ha nell'educazione il suo perno. Il che spiega fra l'altro perché, fra le tante parziali influenze che il positivismo esercita sullo spirito della Terza Repubblica, la più estesa e proficua si faccia sentire nel campo decisivo dell'istruzione pubblica ${ }^{52}$.

\footnotetext{
${ }^{52}$ La trama degli autentici legami fra la cultura positivista e gli uomini politici che governano in Francia durante la prima fase della Terza Repubblica, è argomento storiografico ancora non del tutto sondato. Un aspetto su cui si è già scritto è quello riguardante taluni influssi positivisti avvertibili in Gambetta: lettore del Cours di Comte, amico di Robin, in contatto con Littré, più volte e pubblicamente elogiatore del positivismo, soprattutto per il suo stimolo a porre la scienza al centro delle strategie educative. Ma da qui a ravvisare la presenza di un costante indirizzo positivista nella sua azione di governo, al di là di generiche manifestazioni di stima, ne corre. Più consistenti sono le tracce di positivismo riscontrabili in un altro personaggio politico di primo piano, Jules Ferry, l'artefice principale del profondo riordino e potenziamento dell'istruzione pubblica avvenuto durante la Terza Repubblica. Quando esprime il suo scopo di "organizzare l'umanità senza Dio e senza re", Ferry evoca anche il positivismo, ritenendolo modello di un' arte di programmazione sociale capace di muoversi come via intermedia fra socialismo e capitalismo. E quando, fra il 1881 e l'82, ottiene l'approvazione delle famose "leggi Ferry" che sanciscono il monopolio della scuola pubblica, obbligatoria e gratuita, nel campo dell'istruzione primaria, egli corrisponde ad attese che sono anche dei positivisti, come la sottrazione di tale ramo scolastico al dominio clericale e l'inserimento nei suoi programmi di un spazio maggiore per discipline come la matematica, la storia e la geografia. L'influenza positivista negli indirizzi politici della Terza Repubblica sembra in questo caso più evidente, anche se non esclusiva, visto che la scuola stabilita da Ferry affonda le sue radici anche nella tradizione liberale e repubblicana, da Guizot su fino a Condorcet. Più che col suggerire leggi, i positivisti incidono forse nella riorganizzazione del sapere con il Dictionnaire di Littré: un monumento di politica culturale di larga diffusione popolare, senz'altro lo strumento scientifico più efficace elaborato da un esponente della dottrina positiva. Sull' argomento dei rapporti fra positivismo e Terza Repubblica rinvio a L. Legrand, L'influence du positivisme dans l'oeuvre scolaire de Jules Ferry, Paris, Rivière, 1961; P. Barral, Ferry et Gambetta face au positivisme, in Les positivismes, "Romantisme", cit.; C. Nicolet, Jules Ferry et la tradition positiviste, in Jules Ferry fondateur de la République, Paris, Editions de l'École des Hautes Études en Sciences Sociales, 1985.
} 


\subsection{La "settima scienza" del positivismo: Laffitte e la morale}

Mentre si consuma la scissione del gruppo di Littré dal positivismo comtiano più ortodosso, la fedeltà alle linee tracciate dal fondatore del movimento viene assunta dalla Société Positiviste, sotto la direzione di Pierre Laffitte ${ }^{53}$. Indicato come suo prosecutore dallo stesso Comte, dopo la morte del maestro Laffitte vede rimanere attorno a sé soprattutto la componente operaia del primo nucleo positivista. Mentre gli scienziati preferiscono in maggioranza seguire le strade filosoficamente più blande di Littré, attorno alla bandiera originaria del positivismo decidono infatti di rimanere per lo più gli elementi di estrazione proletaria ${ }^{54}$, più sensibili agli appelli alla rifondazione globale della socialità alla quale li aveva chiamati l'autore del Cours. Per la verità non mancano del tutto gli scienziati, fra coloro che incarnano l'interpretazione più fedele del messaggio comtiano: lo stesso Laffitte è un matematico, e fra i nuovi adepti si affacciano presto altri personaggi di estrazione scientifica, come Jean-François-Eugène Robinet, destinato a diventare un notevole biografo di Comte e di Condorcet ${ }^{55}$. Ma anche la loro adesione al positivismo, al pari di quella degli operai affacciatisi al movimento nel 1848, è motivata più dall'attrazione esercitata dalla religione dell'Umanità che dall'interesse per il discorso scientifico elaborato da Comte.

A partire dagli anni sessanta Laffitte si assume il compito che negli anni quaranta era stato di Littré: quello di maggior divulgatore della filosofia comtiana. In alcune parti del suo pensiero questa "traduzione" è una semplice ripetizione delle tesi di Comte; in altre parti egli riesce invece a essere meno condizionato dall'opera del maestro, a proporne una sintesi stilisticamente più accattivante $\mathrm{e}$ concettualmente più lineare. Il programma originario da cui parte l'azione

\footnotetext{
${ }^{53}$ Le fonti d'epoca intorno a Laffitte, provenienti da suoi collaboratori e discepoli, sono naturalmente di tono elogiativo, ma ricche di informazioni riguardanti il personaggio. Tali sono l'ampia introduzione posta da E. Antoine all'edizione della Morale positive pubblicata da Laffitte presso l'editore Leclerc (Le Havre, 1879), e gli scritti di un autorevole membro della Société positiviste, Georges Audiffrent (M. Laffitte et l'éxecution testamentaire d'Auguste Comte, Paris, Blanchard, 1903), e del positivista inglese Frederic Harrison (Pierre Laffitte, in "Revue occidentale", 1897, pp. 241-254). Valutazioni più recenti e ponderate sono in W.M. Simon, Il positivismo europeo, cit., e in M. Larizza, Bandiera verde, cit., dove si trovano anche altre indicazioni bibliografiche.

${ }^{54}$ L'esponente più noto della componente proletaria in seno al positivismo organizzato è l'operaio specializzato Fabien Magnin, artefice fra l'altro dell'inserimento di altri operai e artigiani di buona qualificazione tecnica nell'associazione di Comte e poi di Laffitte. L'importanza da lui assunta all'interno della compagine positivista è evidente già durante la rivoluzione del 1848, quando firma insieme con Laffitte e Littré il documento politico più importante del movimento (Rapport par la commission chargée d'examiner la nature et le plan du nouveau gouvernement révolutionnaire de la République française, Paris, Mathias, 1848) e viene candidato da Comte a essere uno dei componenti dell'auspicato triumvirato operaio che dovrebbe governare la crisi apertasi in Francia con la rivoluzione. Il ruolo di Magnin rimarrà centrale anche dopo la morte di Comte: fino alla sua scomparsa sarà infatti presidente della Société Positiviste, con Laffitte segretario.

${ }^{55} \mathrm{Mi}$ riferisco in particolare a due opere di Robinet: Notice sur l'oeuvre et la vie d'Auguste Comte, Paris, Au siège de la Société positiviste, 1860, e Condorcet, sa vie, son oeuvre, Paris, Quantin, 1893.
} 
divulgativa di Laffitte si basa sulla distinzione tra "filosofia prima" e "filosofia seconda". La "filosofia prima" rende conto delle leggi astratte della conoscenza e delle leggi universali del mondo; la "filosofia seconda" applica i procedimenti generali alla comprensione degli specifici fenomeni naturali e sociali. Questa seconda parte del progetto di Laffitte non trova per la verità grande sviluppo nelle sue opere, se si fa eccezione per qualche scritto sulla storia dell'umanità, in cui non abbondano però le notazioni originali. Nell' ambito delle applicazioni della filosofia positiva, è tuttavia rilevante il suo ruolo nel ribadire una convinzione affiorata in modo non sempre esplicito nell'ultimo Comte: il completamento della serie delle scienze speciali con una settima scienza dopo la sociologia, vale a dire la morale. E sia nel campo della "filosofia prima", sia in quello delle sue applicazioni particolari, Laffitte si sforza di ricondurre ognuna delle leggi universali del mondo da lui ipotizzate alle sue determinazioni prima in ambito sociologico e poi in ambito morale. Nella sua opera più impegnativa, che è il Cours de philosophie première, Laffitte parte appunto dall'elencazione delle leggi che regolano la conoscenza umana dei fenomeni. In questa rassegna egli cerca di tenere in equilibrio l'impianto empirista del positivismo delle origini con il motivo dell'unità soggettiva elaborato da Comte, che Laffitte difende apertamente dalle critiche di nuova metafisica avanzate da Littré. Da un lato si continuano così a leggere, nella Philosophie première, inviti a subordinare le costruzioni soggettive ai materiali oggettivi e raccomandazioni a non farsi sviare dalle impalcature mentali troppo astratte, perché le immagini interiori sono sempre meno vive delle impressioni esterne (come già aveva affermato l'empirismo inglese del Sei e Settecento). Ma dall'altro, e con maggior insistenza, si tende a costruire un insieme di leggi aprioristiche della conoscenza umana, che finiscono per ingabbiare preventivamente gli indirizzi e gli obiettivi della ricerca scientifica. Tali sono il ribadito rispetto dello spirito d'insieme nella formulazione di ipotesi riguardanti i fenomeni naturali e umani, la concezione delle leggi naturali come immutabili, l'appello alla semplicità e alla "normalità" come tratti preponderanti del corretto funzionamento dell'intelletto. Il tutto è a sua volta subordinato alla nota legge dei tre stadi applicata alla conoscenza, che ci rende partecipi di un progresso della comprensione basato sulla successione di stadi fittizi, astratti e positivi. La rigidità di tipo gnoseologico si accompagna nel pensiero di Laffitte, come vedremo subito, a un'altrettanto solida rigidità intorno alle leggi che reggono la natura, per cui la prima delle finalità della filosofia prima, quella dell'intelligenza, si presenta come fortemente condizionata dai presupposti della dottrina nel suo complesso. Le leggi generali, svelate dal positivismo, costituiscono un confine per la stessa ricerca, che si trova così incanalata in binari predeterminati. Essa deve infatti scegliere di studiare i fenomeni speciali più coerenti con le leggi generali, accantonando come accidentali quelli che non si allineano immediatamente allo spirito d'insieme; deve saper distinguere fra leggi fondamentali e leggi secondarie e selezionare fra immagini mentali corrette e immagini frutto di eccitazione momentanea; deve 
armonizzare la ricerca con lo stato della società, senza trasformare la scienza in un possibile strumento di perturbazione.

L'ultima notazione ci ricongiunge a un altro degli argomenti centrali nell'opera di Laffitte, che è la destinazione pratica della filosofia positiva. "La ragione teorica ha necessariamente per base la ragione pratica. È questa che fornisce il punto di partenza per le sue speculazioni, senza il quale la conoscenza umana si perderebbe in divagazioni indefinite. Ma se la ragione teorica prende il suo punto di partenza dalla ragione pratica, a sua volta reagisce su di essa, per meglio assicurarne l'azione. In definitiva, tutte e due si coordinano e si organizzano verso uno stesso obiettivo, quello del destino umano: organizzare il proprio perfezionamento, per meglio assicurare il servizio degli esseri collettivi: Famiglia, Patria, Umanità, ${ }^{, 56}$.

Il passo appena citato, tratto dal discorso d'apertura anteposto da Laffitte alla Philosophie première, è centrale per la sua argomentazione. Anzitutto viene ribadita la concezione, già comtiana, del positivismo come attività, e non solo come intelligenza. L'attività non è un campo di autonomie: la libertà consapevole è l'adeguamento dei comportamenti individuali alla legge universale del mondo, all'elaborazione della quale Laffitte non per nulla intitola l'intero secondo tomo della Philosophie première. La ragione pratica deve insomma uniformarsi sistematicamente alla legge universale del mondo, che Laffitte, come l'ultimo Comte, interpreta ormai in prevalenza in senso conservatore. La "filosofia prima" non solo ci conferma che la natura è retta da leggi immutabili, ma anche rivela la prevalenza della statica sulla dinamica. Sia nella conoscenza, sia nell'azione l'atteggiamento corretto del positivista è dunque quello della subordinazione delle leggi del movimento a quelle dell'esistenza, effetto della concezione del progresso come sviluppo determinato dell'ordine corrispondente, e non come frutto di una volontà umana incondizionata.

Le conseguenze che Laffitte trae da questo convincimento sono analoghe a quelle di Comte. Nella conoscenza e nell'azione viene anzitutto ribadita la prevalenza dell'elemento collettivo: l'Umanità come destinazione finale di ogni lavoro intellettuale e materiale. Scopo dell'intelligenza non è solo capire, ma anche saper prevedere e modificare l'azione umana perché più intensamente si volga verso i propri destini. Anche Laffitte, come Comte, si lascia andare a questo proposito a schematizzazioni di tipo circolare. Lo scopo degli uomini è quello di vivere per gli esseri collettivi: famiglia, patria, umanità. Per far ciò essi devono perfezionarsi nel corpo e nell'anima, attraverso il sentimento, l'intelligenza e l'azione. Il senso della storia è attraversare con efficacia questo processo precostituito dalle leggi naturali rivelate dal positivismo, che comporta piena coordinazione fra scienza e attività pratica: "Il cammino del lavoro intellettuale

\footnotetext{
${ }^{56}$ P. Laffitte, Cours de philosophie première, t. I, Paris, Bouillon, 1889, p. XL.
} 
sarebbe incomprensibile se non fosse stabilito quello dell'attività umana"57. In questo continuo rinvio tra sfera della conoscenza, scienza del comportamento e produzione materiale, il vero centro dell' argomentazione di Laffitte risulta essere la morale. La stessa legge dei tre stadi, che nella tradizione positivista riveste più finalità, conoscitive e pratiche insieme, nell'autore della Philosophie première si rivela essere soprattutto una linea di individuazione certa del perfezionamento morale nella storia dell'umanità.

La parte più originale del pensiero di Laffitte, per quanto sempre tenacemente attaccata alla filosofia comtiana, è quella che si sforza di teorizzare una specie di etica del sentimento. L'autore della Philosophie première riconosce che la cooordinazione morale fra soggettività e destino collettivo non è un qualcosa di automatico: l'istintività personale è fonte di possibili deviazioni dallo scopo finale, per cui il legame fra teoria e pratica si trova nel costante rischio di porsi in una situazione contraddittoria. Proprio per uscire dal possibile conflitto occorre fare appello alla mobilitazione etica dei sentimenti. "Chiamo funzione composta del sentimento la relazione costante e abituale di una inclinazione del cuore con una veduta dello spirito. Questa funzione composta agisce come una forza semplice che spinge, respinge o mantiene. L'amore degli esseri collettivi è una funzione composta del sentimento, la cui concezione permette di intravedere la possibilità di una legge di evoluzione sentimentale, in rapporto con quelle dell'intelligenza e dell'attività. La necessità di perfezionarsi per il servizio degli esseri collettivi conduce all'evoluzione di sentimenti composti come il controllo di sé, il dovere, ecc." ${ }^{.58}$.

Le tappe di questo perfezionamento, e il suo concludersi nell'utopica armonia di una società positiva compiuta, sono suggerite dall'evoluzione storica conosciuta per il passato e da quella prevedibile per il futuro. Per Laffitte decisiva è stata la formazione del sentimento della famiglia durante l'evoluzione feticistica, agevolata dal culto degli dèi mani che ha ispirato il senso della continuità all'interno del genere umano, e quindi della responsabilità verso chi ci ha preceduto e verso chi ci seguirà. Nel ricongiungersi degli uomini con questa origine risiede fra l'altro il significato più profondo dell'incorporazione del feticismo nel positivismo di cui ha già parlato Comte, e che Laffitte ripropone attraverso l'immagine della Terra "grande feticcio", in quanto luogo dell'espansione dell'Umanità. Il progresso morale ha poi conosciuto un' accelerazione con il medioevo cristiano, con il sorgere dei sentimenti di occidentalità e di onore. Ma è soltanto con il positivismo che i

\footnotetext{
${ }^{57} \mathrm{P}$. Laffitte, Cours de philosophie première, t. II, Des lois universelles du monde, Paris, Au siège de la Société Positiviste, 1894, p. 279.

${ }^{58}$ P. Laffitte, Cours, t. I, cit., p. LIX. A modo loro, Laffitte e i suoi seguaci impostano, sul terreno dei sentimenti, una dottrina di azione e reazione fra singolo e ambiente di tipo psicologico, cercando un'armonia fra pulsioni individuali e voleri sociali, aventi fra i loro scopi anche la protezione della personalità singola. Su questo piano va ricordata in particolare l'opera di Georges Audiffrent, dedito a pratiche di ispirazione positivista che oggi potremmo alla lontana definire di ordine psicoterapeutico, e autore nel 1866 di una Théorie de la vision.
} 
sentimenti composti (nell'elenco di Laffitte sono in particolare sobrietà, pudore, modestia, giustizia, disinteresse, magnanimità, dignità personale) raggiungono la loro più elevata intelligibilità e insieme capacità di moralizzare il mondo.

Nell'età del positivismo "l'evoluzione industriale", ormai avviata al suo definitivo perfezionamento, "crea il sentimento della giustizia, e combinata con la scienza creerà l'amore definitivo per il genere umano; inoltre la scienza, attraverso la modestia, sviluppa il sentimento della dignità personale"59. Laffitte sa che la giustizia nella società industriale, che ha per sua essenza lo scambio equo di prestazioni, non è criterio di cui sia informata l'industria reale, quella dei puri rapporti di tipo capitalistico. Il positivismo ha tuttavia già prodotto, e integralmente, la filosofia scientifica capace di far immaginare una perfezione verso cui ci si può muovere abbastanza rapidamente. Il positivismo è infatti ispiratore e insieme produttore di una "socializzazione sistematica della ricchezza e del lavoro" $" 60$ basata sulla dimostrazione della solidarietà fra gli individui, fra le classi e fra le generazioni.

Di tutta l'indagine di Laffitte sui sentimenti, vale la pena di ricordare in conclusione il ruolo che dovrebbe esercitare la scienza nell'ispirare modestia, anziché superbia intellettuale. La filosofia prima ha infatti, fra gli altri, il compito di ispirare rassegnazione nei confronti delle leggi naturali, contro le quali sarebbe vano e addirittura sciocco ribellarsi. La rassegnazione, secondo Laffitte, non è però nemmeno accettazione inerte del proprio destino, ma adeguamento consapevole e attivo a un ordine collettivo raggiungibile anche attraverso le modificazioni secondarie, che la natura comunque consente in termini di progresso. Il positivismo, con la sua impostazione scientista, ha la decisiva funzione di rendere la nozione di destino una risultante della conoscenza di leggi necessarie, e non più l'effetto dei capricci delle divinità come nelle vecchie teologie, o della sola ragione umana individuale, troppo soggetta a deviazioni dal proprio fine. A questo scopo la rassegnazione deve svilupparsi in collegamento con il principio della venerazione dell'Umanità, che fa accettare ai singoli un ordine decisamente preponderante rispetto ai loro impulsi individuali, ma accolto e anzi amato perché la scienza sa convincere che anche la conservazione degli individui deriva dall'ordine comune ${ }^{61}$.

Il tratto del pensiero di Laffitte appena ricordato ha un seguito nella continuità integrale che la Société Positiviste teorizza e pratica, sotto la sua direzione, con la religione dell'Umanità nei termini ipotizzati da Comte. Da scienziato, Laffitte è consapevole dell'importanza assunta dalle teorie materialistiche dell'età

\footnotetext{
${ }^{59}$ P. Laffitte, Cours, t. I, p. LXI.

${ }^{60}$ Ivi, p. 405.

61 "La filosofia prima è precisamente la sistemazione positiva del destino. Di conseguenza diviene la base della cultura della rassegnazione. Il suo ruolo sociale diverrà in tal modo immenso, sostituendo una rivolta e un'agitazione continue, che spingono a voler cambiare le condizioni necessarie, con una sottomissione fondamentale che servirà da base a una modificazione ragionevole delle cose" $(\mathrm{P}$. Laffitte, Cours, t. II, cit., p. 295).
} 
contemporanea nella spinta alla conoscenza delle verità naturali. Ma il materialismo è per Laffitte, come il razionalismo astratto era per Comte, solo una fase transitoria, storicamente utile per uscire dalle fantasticherie delle vecchie teologie, ma destinata a lasciare il posto alla religione positiva dell'Umanità. Non si tratta peraltro di una semplice credenza collettiva, ma di una vera e propria forma di adorazione organizzata, bisognosa di culti e di militanze di tipo clericale. Non tutti gli aderenti alla Société Positiviste sono entusiasti di questa continuità sistematica con il credo comtiano: Robinet era per esempio perplesso già ai tempi di Comte su una religione che volesse essere, sotto il profilo della ritualità, molto più di una laica celebrazione dei doveri di solidarietà fra gli uomini. Resta tuttavia il fatto che proprio attorno a Laffitte resiste anche in Francia quell'interpretazione quasi mistica del comtismo, che spesso si fa sentire con più insistenza fuori della patria d'origine del positivismo, e in particolare in Inghilterra $^{62}$.

${ }^{62}$ Come in Francia, anche in Inghilterra la cultura positivista si articola in indirizzi non riconducibili a un panorama unitario. C'è anzitutto il positivismo "selettivo" del primo grande diffusore del pensiero comtiano nella cultura anglosassone, l'autore del System of Logic John Stuart Mill. Il grande pensatore liberale apprezza il positivismo come metodo di unificazione delle scienze (ma non come filosofia compiuta) e mantiene una continua simpatia per talune costruzioni concettuali di Comte: fra queste, il carattere fortemente storico dato alla sociologia, la distinzione fra statica e dinamica come strumento per collegare natura e storia, la legge dei tre stadi come terreno di utili suggestioni per connettere la storia dell'umanità con la costituzione della mente umana. Ma egli rifiuta in blocco l'apparato della religione dell'Umanità, nonché la lettura autoritaria e illiberale offerta da Comte dei problemi riguardanti il governo della comunità politica. Sempre nell'ambito della cultura liberale, dovremmo ancora ricordare le tracce di positivismo presenti nel pensiero di Herbert Spencer, nel quale tuttavia, mancando forti continuità con l'impianto di origine comtiana (a favore semmai di quello sansimoniano), il positivismo diventa per lo più un'indicazione generale ad ancorare i ragionamenti etico-politici all'osservazione scientifica e allo sviluppo storico. Un ruolo decisivo per la divulgazione del positivismo comtiano anche oltre i lettori di filosofia (che sono invece il pubblico di Mill e Spencer) esercitano due scrittrici: George Eliot e Harriet Martineau. Il loro positivismo è soprattutto la testimonianza del fascino esercitato da una dottrina morale che si oppone all'anarchia spirituale e insegna la solidarietà fra le classi: un motivo che spiega fra l'altro la simpatia suscitata dal pensiero di Comte, interpretato come una forma etica di socialismo moderato, in ambienti fabiani e in particolare in un'altra donna illustre, Beatrice Webb. Ma il filone del positivismo britannico che va ricordato in special modo nel contesto della nostra ricerca è quello che più rigorosamente si ispira alla religione dell'Umanità. Il personaggio più influente in proposito è Richard Congreve, fondatore nel 1867 della London Positivist Society, forte sostenitore di una religione "dimostrabile" (in quanto basata sulle affermazioni della scienza), ma decisamente orientata verso le pratiche di solidarietà sociale e di vivificazione morale attraverso il culto. Ai margini del gruppo di Congreve, giudicato troppo chiuso in un settarismo tipico delle piccole formazioni religiose, si muove un altro personaggio rilevante per l'interpretazione religiosa del comtismo, vale a dire Frederic Harrison, che abbiamo già ricordato come corrispondente di Laffitte. Harrison, un avvocato al quale si deve lo sforzo maggiore per propagandare il positivismo fra le classi lavoratrici, vede nella filosofia comtiana non tanto uno strumento per comprendere il mondo, quanto un progetto per rendere migliore l'esistenza umana. Fra tutti i continuatori inglesi del positivismo, Harrison è quello che più di tutti ne fa argomento di una costruzione utopica, basata sul raggiungimento di un'armonia universale fondata sull'azione coordinata di ordine e progresso. Sulla diffusione delle suggestioni religiose presenti nel pensiero di Comte in Gran Bretagna rimando comunque a T.R. Wright, The Religion of Humanity: the impact of Comtean Positivisme on Victorian Britain, Cambridge University Press, 1986. 
La circolare inviata nel 1858 ai membri della Société Positiviste, la prima della gestione Laffitte, non lascia dubbi in proposito. La religione dell'Umanità non solo viene indicata come centrale per la testimonianza positivista, ma viene riproposta negli stessi termini rituali teorizzati da Comte. "Soltanto il culto può rendere popolare il dogma, soltanto il culto può renderlo efficace toccando i cuori. [...] Ponendo il culto al di sopra del dogma noi poniamo l'educazione morale al di sopra dell'educazione intellettuale" ${ }^{\prime 63}$. Nella successiva pratica di direzione della Société Laffitte cercherà, dopo questa teorizzazione iniziale, di tenere in equilibrio il carattere filosofico dell'associazione con la tentazione di alcuni suoi collaboratori di farne una vera e propria setta religiosa. La moderazione in questo senso non gli impedisce tuttavia di aggiungere anche proprie immagini all'impianto sacrale di derivazione comtiana, come si può vedere nella parte della Philosophie première in cui evoca le categorie di Spazio, Terra e Umanità. Ognuna di queste nozioni si trova infatti intrecciata con argomentazioni di tipo religioso, a partire dall'idea di Spazio, alla quale corrisponde il culto degli astri. Quanto alla Terra, essa ricopre un peso modesto come parte dell'universo, ma è fondamentale in quanto ambito dell'esistenza umana: da qui la sua definizione di "grande feticcio" degno di adorazione, che già abbiamo incontrato. Infine l'Umanità, comprensiva di patrie e di famiglie, su cui un positivista "ortodosso" come Laffitte non può che ripercorrere le infatuazioni del suo maestro ${ }^{64}$.

\subsection{Il ruolo sociale della scienza: Marcellin Berthelot}

Si è detto, all'inizio di questo capitolo, che esiste un modo di declinare le categorie del positivismo tipico degli scienziati di professione, i quali trovano nei procedimenti della scienza esaltati dal movimento comtiano un argine per contenere l'influenza delle religioni tradizionali. Uno dei rappresentanti più

\footnotetext{
${ }^{63}$ Cfr. W.M. Simon, Il positivismo, cit., p. 49.

${ }^{64}$ Un'ulteriore spia dell'attenzione di Laffitte per le credenze collettive è costituita da un suo opuscolo del 1874, riguardante Considérations générales à propos des cimitières de Paris. Nello scritto Laffitte si schiera dalla parte di coloro che reclamano presso le autorità parigine una maggiore cura nella sistemazione e nella conservazione dei cimiteri, in quanto luogo in cui più intenso può essere il culto dei morti. Tale culto, confermato nella sua fondatezza dalla scienza, che ne rivela l'importanza nel favorire la continuità delle funzioni morali della famiglia, dovrebbe spingere a fare del cimitero un luogo permanente di aggregazione sociale, dello stesso grado del municipio, della scuola e del tempio. Quanto alla generale interpretazione del positivismo come religione, ad essa offrono un contributo decisivo non solo Laffitte, ma anche altri esponenti del movimento: per esempio Georges Audiffrent, autore di un apparentamento fra il positivismo e la predicazione di San Paolo e continuatore, in un'opera del 1903 intitolata La femme, del mito comtiano della VergineMadre. In modo meno radicale e più orientato verso una religiosità civile si muoverà invece il successore di Laffitte alla guida della Société Positiviste, cioè Emile Corra. Nazionalista di tipo umanitario, Corra scriverà fra il primo Novecento e la fine della prima guerra mondiale vari opuscoli dedicati al culto della patria e a quello parallelo dell'umanità, basati fra l'altro ambedue sul culto dei morti.
} 
significativi di questa tendenza è il chimico Marcellin Berthelot. Berthelot non è per la verità un positivista militante: del positivismo condivide più l'atmosfera culturale complessiva che $\mathrm{i}$ tratti specifici del pensiero ${ }^{65}$. Ma egli esprime forse meglio di qualsiasi altro esponente scientifico del suo tempo, quell'ansia di protagonismo sociale della scienza che appartiene a un versante significativo del positivismo $^{66}$. Accanto a questo afflato di carattere scientifico (evocazione nelle sue opere della "scienza positiva"), egli d'altro lato manifesta l'esigenza per l'uomo di aspirare alla perfezione attraverso una lettura filosofica dell'esistenza, da lui rubricata sotto la voce di "scienza ideale".

Nei suoi studi e nelle sue esperienze concrete Berthelot si sforza di delineare l'immagine di un sapiente moderno, e conseguentemente di una scienza moderna, organicamente inseriti nel progresso che riguarda tutti. Da ciò deriva l'attenzione che lo scienziato deve porre, pur dimensionandoli alla propria forma mentale, agli interrogativi tradizionalmente espressi dalla filosofia e alla destinazione economica e sociale del suo operare, sconfinante per forza di cose nell'industria e nella politica. Uno dei suoi avvicinamenti teorici più meditati a questa problematica risiede in una lunga lettera del 1863 inviata a Renan, poi posta come testo di apertura al volume Science et philosophie ${ }^{67}$, e riguardante appunto la distinzione fra "scienza ideale" e "scienza positiva".

Il vero procedimento scientifico, secondo Berthelot, appartiene naturalmente alla scienza positiva, che ha per caratteristiche, come aveva già argomentato Comte nel Cours, il suo procedere per osservazioni e non per assiomi, il suo ancorarsi esclusivamente ai fatti e alle loro concatenazioni dimostrabili, il suo eliminare dall'orizzonte della ricerca elementi non verificabili come l'origine prima e il fine ultimo dei fenomeni. Prendendo a modello gli interrogativi che gli uomini si pongono di fronte a un fatto anche minimo (l'esempio da lui scelto è una torcia che

\footnotetext{
65 Marcellin-Pierre-Eugène Berthelot, nato nel 1827, è uno dei più grandi chimici della sua generazione (morirà nel 1907). Docente al Collège de France fra gli anni sessanta e ottanta, è pioniere degli studi di termochimica e lega il suo nome a esperienze decisive nel campo degli idrocarburi. Fra le sue opere come scienziato, ricordo almeno i manuali di Calorimetrie, Paris, Dunod, 1879, e di Thermochimie, Paris, Gauthier-Villars, 1897. La sua notorietà è confermata dal fatto che alcune sue opere di chimica appaiono tradotte in italiano già entro l'Ottocento. Rimasto ai margini della politica ai tempi del Secondo Impero, ne scopre l'impegno durante la Terza Repubblica, quando, dopo essere entrato all'Institut, diventa senatore. All'inizio degli anni ottanta lo troviamo referente del governo per il riordino dell'insegnamento scientifico di grado universitario: un'esperienza che precede l'assunzione dell'incarico di ministro dell'istruzione pubblica, nella seconda metà dello stesso decennio.

${ }^{66}$ W.M. Simon (Il positivismo, cit.) ricorda Berthelot fra gli scienziati che perpetuano l'immagine comtiana della ricerca di un'unità del sapere come concatenazione ordinata tra fatti, resa possibile dal concetto di spirito d'insieme. Berthelot è tuttavia compreso fra coloro che, scienziati di professione, sono fra i meno convinti delle rigidità filosofiche presenti nel fondatore del positivismo.

${ }^{67}$ Paris, Calmann-Lévy, 1886. Il volume comprende vari scritti di Berthelot usciti a partire dai primi anni sessanta, alcuni d'occasione, riguardanti temi come i fondamenti della ricerca scientifica, l'istruzione, l'origine della morale. Il saggio più impegnativo sotto il profilo teorico è sicuramente quello ricordato come lettera a Renan, intitolato appunto La science idéale et la science positive.
} 
arde), Berthelot costruisce una catena di argomentazioni basate su osservazione, esperienza, comparazione, generalizzazione, che costituiscono l'unico itinerario legittimo di risalita dal singolo fenomeno alle leggi della natura. "Nella costruzione di questa piramide della scienza, tutti i gradini, dalla base alla sommità, poggiano sull'osservazione e sull'esperienza. Uno dei princìpi della scienza positiva è che nessuna realtà può essere stabilita dal ragionamento" ${ }^{, 68}$. La scienza è insomma formata originariamente non dalla concezione, ma dall'esistenza oggettiva dei fenomeni, che essa interpreta secondo conclusioni probabili e mai certe, se non per quella parte che possiamo dare per ragionevolmente acquisita in quanto ampiamente sperimentata nel tempo da parte della comunità degli scienziati ${ }^{69}$.

Fin qui siamo all'interno della più consolidata tradizione galileiana. Berthelot si collega tuttavia a più precise convinzioni positivistiche quando ricorda che il metodo delle scienze positive, nato dall'osservazione della natura si è, a partire dal Settecento, esteso alle scienze politiche ed economiche e al mondo morale. Si tratta di una innovazione del pensiero di importanza cruciale: il programma di estendere i procedimenti della scienza positiva dalla natura alla società "reclama un'attenzione particolare, perché l'estensione universale del metodo positivo è decisiva nella storia dell'umanità, ${ }^{, 70}$. Come vedremo tra poco, questa convinzione autorizza Berthelot a identificare un'origine naturale e non religiosa o comunque metafisica dei comportamenti morali, come suggeriscono i "fatti" oggetto di studio della psicologia, della storia e dell'economia politica. Per quanto riguarda la "scienza ideale" Berthelot, a differenza di Laffitte, non predica rassegnazione di fronte a un'oggettività delle leggi scientifiche, che dal campo della natura vanno sempre più a invadere anche quello dell'uomo e della società. La "scienza ideale", espressione delle libere aspirazioni umane, non va espulsa dal pensiero moderno, ma può trovare ancora un ruolo nel tenere viva l'attenzione e la speranza proprio intorno a quegli elementi di portata assoluta, come il destino finale dell'umanità, che non possono essere catturati dalla scienza positiva, per il carattere relativo che questa riveste. L'affermazione tipicamente scientifica che ciò che esiste ha ragione di essere per il solo fatto di esistere, rischia di appiattire anziché esaltare lo spirito umano, che ha bisogno di credere e di creare, oltre che di constatare. Berthelot si lascia così consapevolmente aperta la strada di una forte idealità, pronta come vedremo a sconfinare nell'utopia. "Nell'ordine intellettuale e morale si verifica

\footnotetext{
${ }^{68}$ M. Berthelot, Science et philosophie, cit., pp. 10-11.

${ }^{69}$ La "verità" scientifica è peraltro il frutto di una ricerca, talvolta lunga nel tempo, che si avvale anche di immagini teoriche e perfino di desideri e aspirazioni morali. È con questo spirito che Berthelot scrive una delle sue opere più diffuse, dedicata alle Origines de l'alchimie (Paris, Steinheil, 1885), di cui ripropone alcuni passi anche in Science et philosophie. E motiva il suo interesse non solo per l'alchimia, ma anche per l'astrologia, come attenzione per il legame storico che intercorre fra progresso dell'ordine scientifico e immaginazione umana, e fra potenza intellettuale e potenza materiale dell'uomo. Les origines de l'alchimie sono oggi disponibili in edizione anastatica: Bruxelles, Culture et Civilisation, 1966.

${ }^{70}$ M. Berthelot, Science et philosophie, cit., p. 13.
} 
qualcosa di analogo a quel che esiste nell'ordine politico. L'esistenza concreta di un governo ideale e assolutamente perfetto è sempre stata a buon diritto considerata chimerica; e tuttavia nessun popolo ha potuto sussistere un solo momento senza un sistema di governo più o meno imperfetto. Nello stesso modo, nell'ordine dell'intelligenza la conoscenza rigorosa dell'insieme delle cose è inaccessibile per lo spirito umano, e tuttavia ogni uomo è forzato a costruirsi - o ad accettare già fatto - un sistema completo, comprendente il suo destino e quello dell'universo" ${ }^{\text {, }}$.

Il ruolo della scienza positiva, continua Berthelot nella citata lettera a Renan, non è dunque quello di servire da negazione della scienza ideale in quanto tale, bensì quello più limitato di spingere verso l'adozione di visioni generali che non contrastino apertamente con i dati dell'esperienza. Per il laico Berthelot ciò significa soprattutto che una scienza ideale moderna non può fare appello all'elemento sovrannaturale, centro delle filosofie antiche: oggi sappiamo che i fenomeni nascono e si riproducono da sé, alcuni anche per effetto della volontà creatrice dell'uomo. Le immagini più appropriate della scienza ideale vanno dunque ricercate nella storia. Nella materia storica è senz' altro difficile rintracciare linee generali, ma non è impossibile: una legge probabile è quella offerta dal "fatto del progresso incessante delle società umane nella scienza, nelle condizioni materiali di esistenza e nella moralità: tre ambiti correlati fra loro" ${ }^{\text {"72 }}$. L'utopia cui Berthelot sceglie di dedicarsi è proprio quella del perfezionamento morale continuo del genere umano, che non può essere certo espresso in termini dogmatici e definitivi, ma può comunque essere comunicato come affinamento indefinito dei grandi sentimenti dell'umanità: l'amore per la bellezza, il culto della verità, la pratica della bontà ${ }^{73}$.

Nello sviluppo del pensiero di Berthelot la speranza sul perfezionamento umano è destinata a diventare un motivo di crescente attenzione ai rapporti fra scienza e morale, come si evince dal titolo di un'altra sua importante opera, appunto Science et morale $^{74}$. L'argomentazione di Berthelot su questo problema ha due finalità. La prima, polemica, è quella di contrastare coloro che ancora in epoca contemporanea pretendono che la morale scaturisca dalle religioni e non dalla scienza. La seconda, costruttiva, è quella di illustrare il ruolo che la scienza può invece esercitare in ambito morale, spingendo l'uomo a migliorarsi nella libertà e non nella costrizione.

\footnotetext{
${ }^{71}$ Ivi, p. 19.

${ }^{72}$ Ivi, pp. 34-35.

${ }^{73}$ Questa aspirazione al perfezionamento dei grandi sentimenti fa evocare anche al laico Berthelot, almeno una volta, il termine Dio, quando descrive la proiezione di tali sentimenti in una "realtà sovrana nella quale questo ideale risiede, cioè Dio, centro e unità misteriosa e inaccessibile verso cui l'ordine universale converge" (ivi, p. 36). È tuttavia evidente che non si tratta di un Dio trascendente, ma di un'immagine scelta per rappresentare l'utopia della perfezione di un'umanità "divinizzata".

${ }^{74}$ Paris, Calmann-Lévy, 1897. Come Science et philosophie, anche Science et morale è composto per lo più da scritti già pubblicati in anni precedenti. Il titolo dell'intero volume è lo stesso del saggio introduttivo composto per l'occasione, che è anche il più meditato sotto il profilo teorico.
} 
È convinzione di Berthelot che la morale, come ogni caratteristica umana, abbia origine dalla natura e non da presunte volontà sovrannaturali. L'istinto di sociabilità è insito nella natura umana, si rafforza con la creazione di istituzioni solidali (dalla famiglia allo stato) e si trasmette di generazione in generazione attraverso eredità storiche. Berthelot non nega il ruolo avuto dalle religioni in questo campo nell'antichità, quando gli uomini non possedevano le conoscenze sufficienti per penetrare davvero nelle leggi della natura. Ma questo non significa che i precetti siano il prodotto della religione o del potere, che in antico si legava strettamente alle concezioni del sacro; significa, più semplicemente, che "l'uomo trova la morale in se stesso e la oggettiva attribuendola alla divinità," ${ }^{, 75}$. Pensare il contrario, riconoscere un ruolo decisivo alle religioni nel guidare gli uomini, è stato più fonte di violenze e di intolleranza che di solidarietà.

L'uomo moderno quindi non deve più fare appello, per la fissazione delle proprie regole di condotta, alla religione organizzata, ma alla scienza, "la sola che possa fornire le basi delle dottrine che troveranno il libero consenso dei cittadini dell'avvenire" ${ }^{, 76}$. Berthelot non entra con questo in sintonia con certi umori comtiani, per esempio con la tentazione di fare degli scienziati i nuovi sacerdoti dell'umanità. Fra sacerdoti e scienziati rimangono differenze insormontabili: i primi tracciano regole assolute ed eterne, i secondi relative e temporanee; i primi elaborano un linguaggio pieno di idolatrie immaginifiche, come l'espressione "essenza delle cose", che i secondi evitano accuratamente; i primi presentano come certe le loro asserzioni, mentre i secondi confessano onestamente che le loro affermazioni sono soltanto probabili. Il carattere relativo della scienza, che oltretutto è giovane rispetto ai culti tradizionali organizzati, non esclude l'eventualità di errori; ma la sua superiorità prospettica in quanto morale è data dalla ricerca di adesione alla realtà naturale, e dal rifiuto della rassegnazione rispetto al volere divino e agli stessi condizionamenti dell'ambiente. Quasi rispondendo a Laffitte, Berthelot dichiara: "Ciò che ha dato credito alla scienza è che, invece di limitarsi a intorpidire i mortali nel sentimento della loro impotenza e nella passività della rassegnazione, essa li ha spinti a reagire contro il destino e ha insegnato loro secondo quale strada sicura possono diminuire la quantità dei dolori e delle ingiustizie, ovvero accrescere la loro felicità e quella dei loro simili""77.

La scienza assume in questa prospettiva un ruolo di liberazione delle energie umane, non disordinata, ma indirizzata a fini generali. Dopo aver recuperato, anche nel testo dedicato a scienza e morale, la distinzione fra scienza positiva e scienza ideale $^{78}$, Berthelot si dedica a elencare i princìpi che l'uomo elabora in sé, scambia

\footnotetext{
${ }^{75}$ M. Berthelot, Science et morale, cit., p. 27.

${ }^{76}$ Ivi, p. XI.

${ }^{77}$ Ivi, p. 3.

78 "In effetti la scienza si presenta a noi sotto un duplice punto di vista: come scienza positiva, che è la base solida di ogni applicazione, nel campo materiale come in quello morale, e come scienza ideale, che comprende le nostre speranze prossime, le immaginazioni, le probabilità lontane" (ivi, p. 14).
} 
e condivide con i propri simili, affida alla coordinazione di istituzioni collettive come lo stato. Questa morale "proclama il dovere, la virtù, l'onore, il sacrificio, la dedizione al bene e alla patria, l'amore degli uomini, la solidarietà"79, non come costrizione autoritaria, ma come elemento di corrispondenza al diritto che ciascuno ha di godere del rispetto e dell'aiuto degli altri uomini. Il carattere positivo della scienza ci dice che tali princìpi sono relativi; ma la persistenza della scienza ideale nell'animo e nella mente degli uomini agita l'obiettivo dei grandi ideali, mai realizzati integralmente ma degni di essere immaginati e rincorsi. Tale fu il valore dell'uguaglianza per gli illuministi e per coloro che promossero la rivoluzione del 1789; e tali sono "le belle speranze per l'avvenire: la fraternità fra i popoli, la solidarietà universale fra gli individui" $\$ 0$.

Il richiamo alla morale ha per Berthelot un valore direttamente politico: "le forze morali, come spesso si è detto, e bene, sono la molla principale che mantiene gli uomini e le nazioni" ${ }^{\prime 81}$. Il collegamento fra etica e politica serve al nostro scienziato anzitutto per motivare la polemica frontale che lo divide dal Secondo Impero. Si tratta fra l'altro di un tema politicamente rilevante per la compagine positivista, che come sappiamo proprio su questo punto si era frazionata tra favorevoli (Comte) e contrari (Littré) al colpo di stato del $1851^{82}$. Il governo di Napoleone III è messo sotto accusa in sé, in quanto regime autoritario e affaristico, $\mathrm{e}$ in relazione alla tradizione dell'Ottocento francese, rispetto alla quale costituisce un calo di virtù civiche e politiche. "Coloro tra noi che sono stati in rapporto con gli uomini del 1830 e della Restaurazione, che hanno potuto intravedere durante l'infanzia gli ultimi superstiti del grande impero e della Rivoluzione, con il loro slancio, l'energia talvolta brutale, l'entusiasmo ardente fino all'accecamento, la superba indipendenza, non hanno sulle cose umane le stesse idee, i giudizi, gli indirizzi della generazione seguente, allevata in uno spirito più positivo, più pratico, forse più egoista, in mezzo al cedimento morale del secondo impero e del culto sfrenato degli interessi materiali, eccitati senza sosta dall'epoca ormai lontana di Luigi Filippo. La tradizione della Rivoluzione fu rotta allora, allo stesso modo in cui l'orgia e la bassezza di Luigi XV avevano fatto dimenticare le alte vedute del regno di Luigi XIV" ${ }^{\prime 3}$.

\footnotetext{
${ }^{79}$ Ivi, p, 29.

${ }^{80}$ Ivi, p. 31. In una lettera a un deputato, compresa anch'essa in Science et morale, Berthelot ha a questo proposito parole di apprezzamento per Giuseppe Garibaldi, fautore come lui dell'ideale degli Stati Uniti d'Europa. Vale fra l'altro la pena di ricordare, in direzione inversa, la simpatia che molti positivisti della Terza Repubblica (anche Littré, oltre a Berthelot) riscuotono negli ambienti repubblicani italiani, perché sostenitori delle società di libero pensiero.

${ }^{81}$ M. Berthelot, Science et philosophie, cit., p. 490.

${ }^{82}$ Per qualche informazione in più sull'importanza esercitata dall'avvento del Secondo Impero sullo schieramento positivista, rimando agli atti del Convegno tenuto a Torino il 30 novembre e $1^{\circ}$ dicembre 2001 sotto il titolo A 150 anni dal colpo di stato di Luigi Napoleone. Bonapartismo, cesarismo e crisi delle istituzioni, e in particolare alla mia relazione sulle Seduzioni della dittatura: positivismo e bonapartismo.

${ }^{83}$ M. Berthelot, Science et philosophie, cit., p. 374.
} 
L'opposizione al Secondo Impero alimenta in Berthelot un sentimento di continuità con i valori repubblicani, democratici, centralistici ${ }^{84}$ e laici. Tiepido con il governo di Thiers, incolpato di sfruttare le vicende della Comune per una reazione antipopolare, il nostro scienziato aderisce invece al successivo consolidamento della Terza Repubblica, stimolandone in particolare l'impegno nell'ambito dell'istruzione pubblica. Il suo coinvolgimento diretto in questo campo, come insegnante e come ministro, trova ovviamente molti riscontri anche nei suoi scritti riguardanti i rapporti fra scienza, filosofia e morale. Di tutto ciò voglio ricordare qui soltanto pochi aspetti, ma particolarmente significativi della sua lungimiranza nel trattare il problema dell'istruzione. Il primo è dato dalla critica persistente agli eccessi di specializzazione negli studi, a favore di un'immagine di scienziato capace di padroneggiare vaste materie e soprattutto in grado di valutare le conseguenze sociali e morali del suo operare. Un secondo aspetto è costituito dalla raccomandazione ai governanti di non farsi condizionare, nel campo dell'istruzione pubblica, dalle attese di rientri economici immediati ${ }^{85}$. Un altro riguarda la chiarezza con cui Berthelot individua nella scuola una doppia funzione, di istruzione professionale e di educazione alla cittadinanza, che è poi quella che autorizza a costruire in quest' ambito un legittimo monopolio dello stato, unico soggetto in grado di coniugare democrazia e libero pensiero e a conferire all'insegnamento un carattere "nazionale, moderno e repubblicano" ${ }^{\$ 6}$.

Mentre molto positivismo del suo tempo si attarda in considerazioni di tipo religioso, il repubblicanesimo di Berthelot si nutre di un deciso spirito laico, con qualche punta di anticlericalismo. Ricordando in ciò più Condorcet che Comte, il nostro scienziato si oppone in forme decise a qualsiasi ingerenza ecclesiastica in ambito politico, e in particolare nel settore dell'educazione pubblica. Nel 1882, in una lunga commemorazione dell'amico Ferdinand Hérold, prefetto della Senna che

\footnotetext{
${ }^{84} \mathrm{Nel}$ settembre del 1870 Berthelot si pone alla testa del gruppo di scienziati che cooperano alla difesa di Parigi di fronte all'assedio prussiano, e sperimenta nell'occasione i problemi provocati dall'interruzione dei rapporti fra la capitale e la periferia della Francia. Ricordando l'episodio, scriverà in seguito, con un'intonazione che va oltre il caso specifico: "La potenza della Francia risiede soprattutto nella sua unità e nella sua forte centralizzazione. Un meccanismo sapiente, organizzato e perfezionato da secoli senza interruzioni, ne riunì le province alla capitale. I cittadini più abili e più istruiti, chiamati da tutte le aree del paese attraverso l'azione delle istituzioni, si trovano riuniti a Parigi. Essi danno l'impulso e il resto li segue, educato da una lunga abitudine. Separare Parigi dai dipartimenti è in qualche modo strangolare la Francia: è l'operazione più terribile e più efficace che si possa realizzare contro di noi" (ivi, p. 428).

${ }^{85}$ A proposito della scarsa propensione del Secondo Impero a investire grandi risorse nell'istruzione, Berthelot commenta: "L'istruzione pubblica è il seme, ma il raccolto si ottiene in altra epoca: nell'ordine morale con l'elevazione generale del livello di civiltà; nell'ordine materiale con la moltiplicazione delle scoperte industriali e con la crescita della scienza e dell'abilità di ingegneri e operai. Ma ci si ostinava allora - e questo pregiudizio non si è ancora dissipato negli ambienti finanziari in cui si regola il bilancio - a cercare a fronte di ogni spesa richiesta per l'istruzione pubblica un'entrata strettamente correlativa. Ora, l'unica entrata delle istituzioni dell'insegnamento superiore consiste nelle iscrizioni e negli esami ..." (ivi, p. 254).

${ }^{86}$ M. Berthelot, Science et morale, cit., p. 64.
} 
aveva avuto il merito di opporsi alla pretesa della chiesa cattolica di mantenere un potere sull'istruzione primaria, Berthelot ribadisce che si può costruire per l'umanità una morale adeguata sulla base della sola educazione generale, senza dover ricorrere a precetti religiosi. Le confessioni non vanno represse, le opinioni dei loro aderenti devono essere rispettate, ma occorre impedire che le religioni prosperino come parassite nel corpo sociale, col pretesto di contribuire a un ordine e a una giustizia che il mondo laico è perfettamente in grado di garantire da sé. "Non si tratta di opporsi ad atti religiosi che la coscienza di un cittadino consideri necessari, quale che sia l'opinione che si può avere al riguardo, bensì di impedire che si imponga a tutti indistintamente la loro pratica nei luoghi pubblici. [...] Il municipio, la scuola, l'ospedale, il cimitero devono essere separati da ogni legame religioso obbligatorio, devono cioè essere puramente laici" ${ }^{\prime \prime}$.

In nome della distinzione fra scienza positiva e scienza ideale, trasferita sul terreno della politica, Berthelot esprime nei suoi scritti più di un apprezzamento per Rousseau. Gli ideali di uguaglianza e di giustizia sociale espressi dal ginevrino sono appunto ideali, non realizzati e forse mai realizzabili integralmente, ma la loro purezza li pone come immagini folgoranti di un perfezionamento morale e politico che va concretamente perseguito nella storia. Rousseau, con Voltaire, è nella convinzione popolare l'ispiratore della Rivoluzione: e se l'obiettivo di Voltaire sulla libertà può dirsi conseguito, non altrettanto vale per la democrazia ugualitaria rincorsa da Rousseau. Per Berthelot tutto ciò significa la necessità di un ritorno, per quanto moderato dall'esperienza storica, allo spirito dei rivoluzionari del 1789. "Certo, noi non dissimuliamo né le lacune, né gli errori di quella impresa grandiosa. Ma è sicuro che essa ha cambiato la faccia del mondo. I suoi risultati nell'ordine civile sono immensi, acquisiti, incrollabili. La maggior parte delle sue idee sulla libertà, la giustizia, l'uguaglianza, la fraternità costituiscono una parte fondamentale dell'essere morale di ogni francese, anzi di ogni uomo civilizzato" ${ }^{\text {. }}$.

Coerente con la sua propensione verso le grandi idealità è infine il modo con cui Berthelot affronta il tema rousseauiano del contratto. Se con questa figura concettuale si intende spiegare la nascita delle società, si erra, perché l'associazione non proviene dalla volontà umana bensì dall'istinto naturale. Però il contratto sociale, se non spiega l'origine dell'unione fra gli uomini, nella sua portata ugualitaria ne rappresenta bene lo scopo: "Anziché essere il punto di partenza, il contratto sociale, cioè il regno della scienza e della ragione stabilito con il consenso volontario del più grande numero, rappresenta al contrario lo scopo finale verso cui l'umanità tende" ${ }^{\prime 89}$.

\footnotetext{
${ }^{87}$ M. Berthelot, Science et philosophie, cit., pp. 406-407.

${ }^{88}$ M. Berthelot, Science et morale, cit., p. 294.

${ }^{89}$ M. Berthelot, Science et philosophie, cit., p. 173.
} 


\subsection{Un mondo governato dalla scienza: sogno o incubo?}

Costante sostenitore del ruolo positivo delle grandi idealità, Berthelot si lascia talvolta catturare dalla tentazione di formulare vere e proprie immagini utopiche. Avviene in particolare il 5 aprile 1894, a un banchetto della Camera sindacale dei prodotti chimici, quando il nostro scienziato pronuncia un'orazione volta a delineare quale potrebbe essere il mondo Nell'anno 2000 (con questo titolo il discorso sarà pubblicato in Science et morale). Berthelot riconosce che i sogni degli uomini sono destinati a realizzarsi solo parzialmente nella storia, ma questo non toglie significato al desiderio di descrivere un cammino verso la felicità, frutto dell'azione umana congiunta col progresso delle scienze.

Il futuro che Berthelot immagina sarà appunto l'effetto combinato dell'intelligenza umana, dell'industria e del lavoro. Nella sua visione le scienze fisiche, applicate alla produzione, sapranno appropriarsi dell'energia solare e di quella interna della terra, mentre le scienze chimiche metteranno a disposizione una gamma incessante di prodotti costruibili senza grandi sforzi. I mezzi di comunicazione consentiranno alle merci di circolare con rapidità, e lo stesso avverrà per le persone (Berthelot intuisce la futura espansione del volo su scala planetaria), con positive ricadute anche sui rapporti fra nazioni e culture diverse. $\mathrm{Ne}$ risulteranno vantaggi enormi per il lavoro, ridotto come durata e soprattutto come fatica, e per l'ambiente, con l'eliminazione degli effetti inquinanti provocati dalle fabbriche e dalle miniere che caratterizzano finora la civiltà industriale conosciuta. "La terra diverrà un vasto giardino, bagnato dalle effusioni delle acque sotterranee, nel quale la specie umana vivrà nell'abbondanza e nella gioia della leggendaria età dell'oro" $"$.

Come è consueto negli scritti di Berthelot, il progresso della felicità futura non sarà affidato alla semplice egemonia delle applicazioni tecniche e scientifiche, ma comporterà trasformazioni positive di ordine etico e politico. La maggiore produzione e circolazione delle ricchezze, unita alla migliore coordinazione fra $\mathrm{i}$ popoli, offrirà per esempio uno spazio crescente alla cooperazione internazionale, fino a che giungerà il momento in cui "non ci saranno più dogane, protezionismo, guerre, frontiere arrossate dal sangue umano" ${ }^{\text {". }}$. La pace internazionale sarà un prolungamento della pace interna, della collaborazione fra le classi, che sarà anch'essa agevolata dalla tecnologia e dalla scienza. Berthelot sa peraltro che

\footnotetext{
${ }^{90}$ M. Berthelot, Science et morale, cit., p. 514. L'utopia di Berthelot sarà oggetto di una rispettosa attenzione, all'inizio del Novecento, da parte di Anatole France. S. Rota Ghibaudi (L'utopia e l'utopismo. Dalla grande progettualità al ripiegamento critico, Milano, Angeli, 1987, p. 51) rileva che per France Berthelot è, con Renan, fra coloro che evidenziano una "disposizione mentale a ricercare nel presente e nel passato le condizioni dell'avvenire", attraverso i procedimenti dell'utopismo scientifico.

${ }^{91}$ M. Berthelot, Science et morale, cit., p. 510.
} 
l'equilibrio sociale e la soddisfazione generalizzata dei bisogni non sono un prodotto automatico dello sviluppo economico, ma richiedono un' appropriata veste politica e morale. E con una vena di ironia il nostro scienziato ipotizza che "saremo pronti a realizzare i sogni del socialismo, posto che si riesca a scoprire una chimica spirituale che cambi la natura morale dell'uomo, con la stessa profondità con cui la nostra chimica trasforma la natura materiale" $"$. Se una notazione del genere sembra prendere le distanze dall'ipotesi di rifondare moralmente l'uomo, Berthelot è comunque in grado di avanzare un suggerimento per cominciare a rendere migliore la società. Si tratta della tutela del lavoro, praticata da un'organizzazione della vita economica su cui lo scienziato non offre indicazioni particolareggiate, ma vicina per aspirazioni agli ideali del socialismo democratico. "L'uomo che lavora è buono, il lavoro è la fonte di ogni virtù. In questo mondo rinnovato ognuno lavorerà con zelo, perché gioirà del frutto del suo lavoro, e troverà in tale remunerazione legittima e integrale i mezzi per spingere al più alto livello il proprio sviluppo intellettuale, morale ed estetico" ${ }^{\text {"93 }}$.

Nella ricerca che stiamo sviluppando, l'utopia un po' elementare di Berthelot serve non solo per completare il quadro delle tendenze dello scientismo postcomtiano, ma anche per introdurre una problematica culturale che verrà sviluppata nei prossimi capitoli, aventi a che fare direttamente con le operazioni utopiche condotte intorno al binomio scienza-storia, ragione-tempo. Come vedremo, una tematica del genere si presenta già nell'epoca del positivismo in ascesa come controversa, soggetta a speranze ma anche a perplessità e paure sull'idea di un mondo governato da una razionalità scientifica: paure che, come gli entusiasmi, sono sovente rappresentate attraverso costruzioni letterarie prossime all'utopia. Ma anche al di fuori della scrittura utopistica appaiono dubbi di segno antiscientista, dei quali ricordo qui qualche espressione, a cominciare dalle opinioni di un personaggio illustre già citato per i legami con Berthelot, Ernest Renan, con il quale il nostro scienziato intrattiene una relazione epistolare lunga una quarantina d'anni.

Non è possibile in questa sede costruire un profilo attendibile, neppure per sommi capi, di un'opera intellettuale complessa e articolata come quella di Renan, spesso apparentato al positivismo per qualche suo apprezzamento del ruolo progressivo della scienza, ma collegabile anche, e in modi talvolta più proficui, agli umori di altre correnti culturali del suo tempo ${ }^{94}$. L'interesse per una storia segnata dal concetto di spirito nazionale e la convinzione intorno alla superiorità del

\footnotetext{
${ }^{92}$ Ivi, pp. 510-511.

${ }^{93}$ Ivi, pp. 514-515.

${ }^{94}$ Per una visione più approfondita del personaggio, rinvio a qualche studio monografico. Fra i meno lontani nel tempo, ricordo K. Gore, L'idée de progrès dans la pensée de Renan, Paris, Nizet, 1970; L. Rétat, Religion et imagination religieuse: leurs formes et leurs rapports dans l'oeuvre de Renan, Paris, Klincksieck, 1977; H.W. Wardman, Renan. Historien, philosophe, Paris, Editions d'enseignement supérieur, 1979.
} 
sapiente lo uniscono infatti alla tradizione romantica, mentre lo scetticismo sulla plausibilità delle filosofie universalistiche lo mettono in relazione con il nascente decadentismo europeo. In questo insieme complicato di suggestioni il problema del ruolo presente e futuro della scienza (e dello scienziato) trova comunque un risalto particolare: e solo di questo farò ora cenno.

Il rapporto fra Renan e la scienza come problema politico attraversa fasi abbastanza distinte ${ }^{95}$. La prima è quella racchiusa soprattutto nelle pagine dell'Avenir de la science: opera scritta nel 1848, frutto quindi delle esaltazioni rivoluzionarie di quel momento, ma anche pubblicata soltanto nel 1890, e fra mille avvertenze ed esitazioni da parte del suo autore. L'Avenir de la science è il testo di Renan di più marcata intonazione positivista (per la precisione più sansimoniana che comtiana), nel quale si auspica l'assunzione da parte degli scienziati di una vera e propria funzione di direzione politica. Per l'immediato (del 1848) Renan fa appello al sapiente (che non è necessariamente uno scienziato) perché dia la parola al popolo in rivolta, secondo un motivo derivato da Michelet (altro personaggio a lui presente, come Saint-Simon). Il risveglio delle masse attraverso idee e passioni prestate loro dall'intellettuale non prelude tuttavia, nelle attese di Renan, alla costruzione di una società democratica, ma casomai di una specie di socialismo nazionale governato dai tecnici e dagli scienziati. Radicalizzando un motivo sansimoniano, Renan sogna l'avvento non solo di un regime che si serva delle competenze scientifiche, ma addirittura di un sistema diretto in prima persona dagli scienziati, che come ceto dirigente varrebbero senz'altro di più delle vecchie aristocrazie nobiliari o delle nuove élites selezionate con il metodo poco qualitativo del suffragio universale. "Il governo ideale sarebbe un governo scientifico, in cui uomini competenti e straordinari trattassero le questioni di governo come problemi scientifici e ne cercassero la soluzione razionalmente. Finora sono stati in genere la nascita, l'intrigo o il privilegio del primo occupante a conferire i gradi ai governanti" ${ }^{\prime 96}$.

Il fallimento del 1848, sancito significativamente dall'ascesa al potere di Luigi Napoleone col voto popolare, apre sul tema del governo una seconda fase della riflessione di Renan, destinata a durare per tutto il Secondo Impero. I sentimenti contrari all'eventualità di affidarsi alla volontà popolare tendono ora a esacerbarsi, nella convinzione che gli uomini sono segnati da una profonda diseguaglianza naturale fra una minoranza di spiriti eletti e una massa motivata da obiettivi limitati. La democrazia conduce inevitabilmente a un appiattimento delle opinioni,

\footnotetext{
95 Per le scansioni interne al pensiero politico di Renan traggo ispirazione dal bel saggio di B. Casalini, Ernest Renan: la rappresentanza della ragione, in AA. VV., Il popolo e le élites, cit., pp. 75-94. Alla Casalini dobbiamo anche una recente traduzione del Calibano di Renan (Palermo, Sellerio, 1995), preceduta da un'introduzione nella quale sono presenti cenni al problema che qui ci interessa in particolare, quello dei rapporti con la cultura scientifica.

${ }^{96}$ E. Renan, L'avenir de la science, in Oeuvres complètes, a cura di H. Psichari, Paris, Calmann-Lévy, 1947-1961, vol. III, p. 1007.
} 
che costituisce la cornice culturale entro cui si rafforzano e si diffondono sentimenti collettivi di portata in prevalenza materialistica. In contrasto con le speranze del 1848, il Renan di questo periodo reputa i sapienti poco adatti a governare, perché la virtù si può al massimo praticare, non insegnare. La scienza, e l'attività intellettuale in genere, sono il frutto di una ricerca personale e appartata: per il ruolo di aristocrazia sociale è meglio affidarsi ad altri soggetti, più abituati a interagire col mondo politico. E un Renan che sta abbandonando le passioni progressiste della giovinezza per sentimenti sempre più conservatori, riscopre per un momento, dal suo punto di vista, le potenzialità stabilizzatrici della nobiltà di nascita. Verso il termine del Secondo Impero egli vede in un ritorno di funzioni affidate a questa classe uno degli strumenti possibili di una svolta monarchicocostituzionale, auspicando implicitamente l'adozione anche in Francia del modello "inglese" $"$.

Le diffidenze renaniane nei confronti della democrazia, ma anche delle capacità dei sapienti di governare in prima persona il processo storico, precipitano al tempo della Comune in un ritrarsi spaventato. Il popolo è un mostro al quale il saggio dà la parola, ma col pericolo costante di poterne poi essere divorato, secondo una tematica che troverà espressione qualche anno dopo nel Caliban, integrazione della Tempesta di Shakespeare. Il disorientamento di Renan è bene espresso letterariamente nella prosa dei Dialogues philosophiques, pubblicati nel 1871, nei quali il problema del ruolo politico del sapiente viene più volte evocato, in termini ormai lontanissimi dall'entusiasmo quarantottesco. Da un lato si ribadisce che lo scienziato non è un soggetto adatto al governo politico anche se è mosso dalle migliori intenzioni di perseguire l'interesse generale, perché la guida degli uomini presuppone una capacità di intervento "mondano" che gli asceti della ricerca non possono avere. Dall'altro si agita il sospetto che anche il governo degli scienziati possa essere una pura operazione di potere, il che produrrebbe effetti spaventosi quando l'autorità politica si unisse con la superiorità intellettuale. Emerge da qualche pagina dei Dialogues un'immagine che è l'esatta antitesi del sogno di un mondo pacificato dalla scienza, ma comunica anzi il possibile dominio futuro della scienza come un incubo. Forti delle proprie conoscenze, inaccessibili per la loro complessità alla maggioranza degli uomini, pochi scienziati potrebbero un domani essere in grado di minacciare il mondo intero con gli strumenti di morte da loro creati, e perpetuarsi come razza superiore costruita da una selezione artificiale. È evidentemente un'immagine anch'essa di intonazione utopica, seppur di segno negativo, con la quale tuttavia Renan colpisce al cuore le ambiguità della scienza moderna, strumento di liberazione ma anche di esclusione, e della tecnica moderna,

\footnotetext{
${ }^{97}$ Il riferimento è alle opere di Renan La monarchie constitutionnelle en France (1869) e La réforme morale et intellectuelle de la France (1871), ripubblicate nelle citate Oeuvres complètes, rispettivamente nei vol. II e I. Della Réforme esiste una recente traduzione italiana: E. Renan, La riforma morale e intellettuale della Francia, a cura di R. Pozzi, Roma, Istituto storico italiano per l'età moderna e contemporanea, 1991.
} 
produttrice del miglioramento della vita umana ma anche dei mezzi per la sua distruzione. La macchina campeggia spesso, in questa fase della scrittura renaniana, come strumento di morte, nella sua forma evidente in quanto armamento ma anche come metafora di una possibile condizione permanente di asservimento dell'uomo a un mondo costruito dalla tecnica. Il quale mondo avrebbe come forza in più, rispetto alle dittature del passato, l'impossibilità di controllare e modificare la volontà dei pochi detentori di un potere scientifico basato su conoscenze sempre più specializzate, che né le masse (ovviamente), ma nemmeno i sapienti di genere umanistico saprebbero più dominare.

Anche la posizione che vede nelle gerarchie del passato nobiliare una possibile difesa dalla cultura quantitativa del presente, non è tuttavia definitiva nel pensiero di Renan. Trascorsa la crisi del 1870, assistiamo infatti a un lento per quanto scettico riavvicinamento alla tematica repubblicana, sotto la specie di un riconoscimento tacito ma effettivo dei fondamenti della Terza Repubblica. La democrazia parlamentare continua a essere giudicata un sistema pieno di difetti, ma essa presenta almeno la caratteristica di non essere fra i regimi più intenzionati a reprimere la libertà di pensiero e di ricerca. D'altra parte si presuppone che la scelta degli elettori non cada sui tecnici e sugli scienziati come corpo separato, ma sui politici di professione, come avviene di fatto durante la Terza Repubblica. L'auspicata libertà della cultura consente ora agli scienziati, e agli intellettuali in genere, di dedicarsi ai loro compiti precipui, quelli di conoscere il mondo e di esprimere opinioni. Il ruolo del sapiente si conferma non essere quello di governare in prima persona le società politiche, ma egli ha egualmente due funzioni civili, seppur più limitate, da perseguire. Può darsi da fare per correggere gli errori inevitabili del suffragio universale senza negarlo apertamente, ma circondandolo con riforme che facilitino l'emergere di un'aristocrazia dei migliori in un regime democratico $^{98}$. E può corrispondere con la sua attività al bisogno, che anche un sistema democratico e repubblicano ha, di processi di sacralizzazione della politica, di forte identificazione passionale fra cittadini e istituzioni: una missione che Renan si assume soprattutto nella veste di suscitatore dell'identità nazionale ${ }^{99}$.

Una posizione intermedia fra l'entusiasmo scientista di Berthelot e talune asprezze apocalittiche di Renan intorno a un possibile governo degli scienziati, assume, a cavallo fra Otto e Novecento, un altro personaggio che si confronta con i

\footnotetext{
${ }^{98}$ Renan indica come possibili rimedi agli inconvenienti qualitativi del suffragio universale le elezioni a due gradi e l'istituzione di una camera alta. Si può ricordare che anche Stuart Mill, nel testo del 1861 sul Governo rappresentativo, prevedeva correttivi al voto uguale, giudicato non in grado di per sé di assicurare la scelta dei "migliori". Tali correttivi erano costituiti soprattutto dal cosiddetto "voto plurimo" (possibilità per l'elettorato più colto di votare in più collegi) e dall'attiva presenza, accanto al parlamento, di una commissione di esperti incaricata di preparare le leggi con la necessaria competenza: leggi che l'assemblea avrebbe potuto approvare o rigettare, ma non modificare.

99 Vedi in proposito soprattutto l'orazione tenuta alla Sorbona nel 1882, di cui possediamo un'edizione italiana recente: E. Renan, Che cos'è una nazione?, intr. di S. Lanaro, Roma, Donzelli, 1993.
} 
temi qui evocati: Emile Faguet. Grande critico letterario e storico della cultura, professore alla Sorbona e accademico di Francia, Faguet si avvicina solo da giovane, e in termini abbastanza marginali, alla cultura del positivismo. Il suo approdo è un altro, quello rappresentato dalla "Revue des Deux Mondes" sotto la direzione, a fine secolo, di Ferdinand Brunetière, col quale, come con la rivista, Faguet collabora assiduamente ${ }^{100}$. E come Brunetière, anch'egli esprime a cavallo fra Otto e Novecento il desiderio del ritorno a uno spiritualismo tradizionalista, aperto al dialogo con le religioni convenzionali, polemico nei confronti dell'illuminismo per quella parte in cui credeva alla possibilità di creare uomini nuovi usando come strumento principale la diffusione della ragione.

Il razionalismo moderato praticato da Faguet, venato di scetticismo e sorvegliato da richiami moralistici di segno conservatore, non sembra lasciare molto spazio all'apprezzamento per la forte fiducia nella scienza manifestata dal positivismo. Eppure il nostro professore, ancora nel 1911, riconoscerà quasi con compiacimento che il suo maestro Brunetière "ha cominciato con l'essere positivista, con l'essere, come eravamo praticamente tutti a quell'epoca, profondamente influenzato da Auguste Comte. Del resto né lui né io abbiamo mai rinnegato questo grande maestro" ${ }^{\text {"101 }}$. Comte, agli occhi di Faguet, fu un grande maestro perché all'inizio aveva opportunamente escluso dall'ambito della scienza quel che la scienza non può offrire: la risposta agli interrogativi sul senso finale dell'uomo e del mondo, materia razionalmente inconoscibile che non può essere argomento di scienza. Faguet è peraltro consapevole che l'opera "negativa" del positivismo, tesa potenzialmente a separare scienza e metafisica, è però convissuta in Comte con l'aspirazione contraria a fare della scienza stessa una fede, un sistema compiuto capace di rispondere a ogni pensiero e a ogni sentimento umano ${ }^{102}$. Comte è stato

\footnotetext{
${ }^{100}$ Vissuto fra il 1847 e il 1916, Emile Faguet è allievo dell'Ecole normale supérieure, poi insegnante liceale e giornalista letterario, fino ad approdare negli anni novanta alla cattedra di poesia francese alla Sorbonne. Fra le sue opere di storia culturale, ricordo in particolare Politiques et moralistes du dix-neuvième siècle, Paris, Lecène, 1899. Un profilo esauriente dei suoi interessi culturali è in M. Duval, Emile Faguet: le critique, le moraliste, le sociologue, Paris, Lecène, 1911. Quanto ai rapporti di Faguet con la religione, dopo una formazione cattolica abbandona le religioni rivelate, sulla spinta della lettura della Vie de Jésus di Renan, per mantenere un atteggiamento prudentemente agnostico ma rispettoso della tensione verso il sovrannaturale presente nell'idea di spiritualità; salvo "riconvertirsi" al cattolicesimo poco prima della morte.

${ }^{101}$ E. Faguet, Ferdinand Brunetière, Paris, Hachette, 1911, p. 17.

${ }^{102}$ In un'interessante opera di sistemazione del proprio percorso dottrinario, uscita nel 1912 con il titolo Initiation philosophique (Paris, Hachette), Faguet scrive a p. 158 che Comte "ha costruito una filosofia soprattutto negativa, malgrado la sua grande pretesa di rimpiazzare la negazione del XVIII secolo con una dottrina positiva". L'opera di demolizione comtiana è consistita soprattutto nel togliere credibilità ai dogmi delle religioni tradizionali, e su questo punto ha saputo ispirare "il credo, o piuttosto il non credo, di un numero abbastanza grande di intelletti". Altro e più fallimentare è il discorso riguardante il positivismo come dottrina ricostruttiva. Già parecchi anni prima, nel ritratto dedicato a Comte nei Politiques et moralistes, Faguet scriveva che Comte "ha avuto nell'avvenire della scienza, nella sua preponderanza finale, nella sua pretesa di bastare allo spirito umano e di poter governare l'umanità, una fiducia forse eccessiva; e il positivismo non è parso capace di tutto ciò che
} 
un grande maestro anche in un altro senso: nell'insegnare l'importanza che ricopre la conservazione della società per la vita degli uomini, e nello spiegare come la loro libertà debba essere per necessità tenuta sotto controllo dalle esigenze della compattezza comunitaria. Faguet è in proposito tra i fautori di quel ritorno alle tradizioni come cemento unitario, che contrassegna gran parte del conservatorismo ottocentesco. E, come Brunetière, e come di lì a poco gli epigoni nazionalisti del comtismo, ama ricordare con soddisfazione l'insistenza con cui l'ultimo Comte proponeva la sacralità del culto dei morti.

Il legame inevitabile fra pulsioni alla libertà individuale e sue giuste limitazioni in nome della comunità non si spinge in Faguet fino a perpetuare l'intenzione di molti positivisti di descrivere l'umanità secondo l'idea di organismo sociale, sul quale si possa imporre una linea improntata al determinismo storico ed etico. La coscienza del "sociale" di derivazione positivista (oltre che tradizionalista, s'intende) serve comunque a criticare le categorie di libertà e di uguaglianza, quando siano radicalizzate. La libertà individuale non può essere teorizzata come svincolata dalle esigenze comunitarie, nelle quali trova concretezza, così come l'uguaglianza non può essere enfatizzata fino a cancellare differenze che risiedono nella natura e nella storia. L'idea di uguaglianza, scriverà in un saggio del 1902 dedicato al Libéralisme ${ }^{103}$, presenta aspetti positivi, perché con essa si rinforzano la considerazione di sé e il sentimento della giustizia, ma anche negativi, perché nella coscienza di molti prelude all'odio per il superiore e a un collettivismo intenzionato a cancellare ogni distinzione di merito.

Il tradizionalismo che si respira in molte pagine di Faguet, lo spinge naturalmente a rifiutare ipotesi di rifondazione del mondo su basi scientiste. $\mathrm{Su}$ questo punto egli perpetua $\mathrm{i}$ dubbi già presenti in Renan (un altro dei suoi riferimenti dichiarati, insieme con Taine), esprimendoli tuttavia più con argomentazioni di ordine storico e realistico che attraverso immagini distopiche. In una delle sue opere più importanti sotto il profilo politico, la raccolta di saggi intitolata appunto Questions politiques, del 1899, appare uno scritto che ci riconduce direttamente al problema che stiamo qui discutendo, e nel quale il suo autore si interroga su cosa sarà il XX secolo. La prima risposta che si dà Faguet è che il XX secolo sarà probabilmente il secolo della scienza, ma non per questo potrà essere automaticamente etichettato come il secolo del progresso. Lo sviluppo della scienza produce di solito un'analoga accelerazione nella tecnica e quindi nella produzione, ma tutto ciò non si identifica con il progresso morale: non sempre, anzi raramente, l'evoluzione materiale si accompagna con il progresso etico, e non invece con la decadenza morale, estetica, di costume. "Il pensiero non si accumula, il genio non si accumula, ma il sapere si accumula": da ciò deriva la giusta

egli lo riteneva in grado di fare, né di soddisfare completamente la dimensione spirituale dell'uomo" (Politiques et moralistes du dix-neuvième siècle, cit., t. II, p. 367).

${ }^{103}$ Paris, Lecène. 
considerazione che le conoscenze scientifiche dell'umanità aumentano incessantemente. Ma "questa idea di progresso, che è vera in campo scientifico, l'umanità non ha esitato ad applicarla a tutti gli ambiti, credendo vera la perfettibilità di tutte le cose" ${ }^{\prime 104}$ : che è invece tutt'altro argomento.

Secondo Faguet la scienza moderna, con la sua indagine desacralizzata sulla natura e sull'uomo, ha contribuito a diffondere il sentimento della democrazia; con le applicazioni della tecnica ha conferito alle nazioni un potere di distruzione del nemico più intenso che in passato; con il suo intreccio con la produzione ha posto le basi per l'autorità di una nuova élite, più incerta e fluttuante della nobiltà tradizionale, che Faguet definisce plutocratica. Tutto ciò non è né miglioramento morale, come si è detto, né pacificazione del genere umano. Il futuro affidato alla scienza è un avvenire pericoloso, portatore di nuovi conflitti imperialistici e di classe, se non interverranno nella politica e nell'etica correttivi capaci di porre anche la scienza sotto il controllo della tradizione.

Nel già ricordato libro dedicato al Libéralisme, le critiche alla democrazia pura di Faguet si volgono in positivo sotto forma di auspicio per un sistema politico futuro più ancorato del presente a sentimenti conservatori. La superiorità dello stato rispetto a ogni altra forma di autonomia, individuale e di gruppo, è indiscutibile. Ma per evitare che la direzione dello stato sia affidata alle incertezze del suffragio universale, che presenta scarse garanzie in merito al riconoscimento dei valori morali, occorre anzitutto porre correttivi di ordine "aristocratico" al sistema elettorale e istituzionale ${ }^{105}$. Più che alla politica in senso stretto, la conservazione del costume sociale è tuttavia affidata ai rapporti fra lo stato e quelle che Faguet chiama "aristie": luoghi di parziale autonomia economica, sociale e culturale, ma senza esercizio in proprio di autorità politica, che equilibrino le inevitabili tendenze livellatrici del mondo tecnologico moderno. Seguendo un motivo presente anche in Taine, Faguet reputa che, più che agli individui, questa autonomia vada riconosciuta alle associazioni, più capaci di frenare le spinte disgregatrici del personalismo eccessivo, all'interno di un sistema gerarchico e con qualche intonazione corporativa. Se il quadro generale viene ricomposto secondo linee di prevalente ordine conservatore, anche la scienza può ritrovare una funzione apprezzabile nella società del futuro (che è un po' anche quella del passato) auspicata da Faguet. Fra le associazioni delle classi elevate l'autore del Libéralisme

\footnotetext{
${ }^{104}$ E. Faguet, Questions politiques, Paris, Lecène, 1899, p. 296.

${ }^{105}$ Come per Renan, un ruolo significativo nel far emergere le opinioni dei ceti più istruiti potrebbero esercitare, secondo Faguet, le elezioni a doppio grado, che affidano la scelta definitiva dei rappresentanti ad assemblee di ridotte dimensioni, nelle quali di solito prevalgono le figure dei notabili. Per quanto ridimensionato nel suo significato immediatamente democratico dall' esistenza del secondo grado, il suffragio popolare andrebbe comunque ampliato con il riconoscimento del diritto di voto alle donne. Altro modo di imbrigliare le assemblee elettive potrebbe essere a suo parere quello di prevedere nella carta costituzionale la possibilità di una limitata cooptazione di soggetti meritevoli, al di fuori del voto popolare (un meccanismo simile a quello oggi in vigore nel nostro ordinamento per i senatori di nomina presidenziale, ma molto più diffuso nei senati ottocenteschi).
} 
Dopo Comte: positivismo moderato e positivismo radicale

cita infatti con particolare insistenza le accademie scientifiche e quelle culturali, accanto alle compagnie industriali, mentre le classi basse troveranno la loro identità soprattutto all'interno delle cooperative e dei sindacati. 



\section{La "macchina del tempo"}

\subsection{Renouvier e il fascino della "ucronia"}

Assumere la costruzione della "macchina del tempo", ossia del viaggio nel tempo codificato in ambito letterario da George Herbert Wells, come una delle linee interpretative dell'intreccio fra scienza e utopia nell'età del positivismo, è una scelta giustificata anzitutto dalle cronache culturali del periodo. Ancora prima della pubblicazione di The Time Machine, e in forme ovviamente più intense dopo, la tendenza a usare gli strumenti dell'utopia per immaginari spostamenti nella storia coinvolge infatti parecchi scrittori, nella patria di Wells come nella vicina Francia. E lascia, come vedremo in questo capitolo, consistenti tracce che, per i loro dialettici legami con lo scientismo dell'epoca, costituiscono una parte rilevante della vicenda delle declinazioni utopiche del messaggio positivista.

Un primo elemento di continuità fra la cultura positivista e l'utopia del viaggio nel tempo è rintracciabile nel peso assunto dalla scienza e dalla tecnica nelle descrizioni immaginarie del futuro, che costituiscono l'aspetto più rilevante, anche se non il solo, della "ucronia". La centralità, in positivo o in negativo, ricoperta in tale utopia dall'esistenza di un mondo fortemente tecnico e scientifico sarebbe impensabile al di fuori dell'atmosfera culturale creata nella seconda metà dell'Ottocento dal positivismo. Un altro elemento ricavabile dalla tradizione comtiana, e che la letteratura della "macchina del tempo" riproduce, è la convinzione di poter prevedere e raccontare lo sviluppo della realtà nel futuro. A differenza che all'epoca di Comte, questa operazione assume a fine secolo i toni espliciti dell'utopia letteraria, mentre nel fondatore del positivismo era argomentata in sede trattatistica.

L'ucronia, come genere di scrittura utopica, si presenta prevalentemente nella forma della descrizione immaginaria del futuro, quasi sempre frutto, sul piano letterario, di un "sogno" raccontato dal narratore-protagonista dopo il suo 
risveglio ${ }^{106}$. L'uso delle tecniche "ucroniche" per rappresentare un viaggio immaginario non è però esclusivo della direzione del futuro: qualche volta lo spostamento nel tempo assume la veste del ritorno al passato. Ed è quanto accade proprio a uno dei pionieri dell'ucronia, Charles Renouvier, che la definisce appunto "utopia dei tempi passati"107.

Renouvier opera ai margini, se non al di fuori del movimento positivista ${ }^{108}$. Allievo di Comte all'École Polytechnique, non ne ricava un ricordo celebrativo; e anche in riferimento alla cultura positivista nel suo complesso, dopo averne in qualche modo accettato il programma di limitare la ricerca filosofica al conoscibile in termini sperimentali, se ne distacca rapidamente, per costruire un itinerario di riflessione dai caratteri abbastanza personali, per quanto largamente debitore nei confronti di Kant. Nelle opere di Renouvier di carattere più propriamente filosofico, un vago sentore positivista è riscontrabile solo all'origine dell'argomentazione, quando egli pone al centro della sua ricerca la categoria di "relazione" tra fenomeni finiti, e con ciò ribadisce il concetto relativistico della speculazione filosofica ${ }^{109}$. Lo sviluppo del suo pensiero abbandona tuttavia le

${ }^{106}$ Ricordo che la prima espressione di tale genere letterario si era avuta in Francia già nel secolo precedente, e precisamente nel 1770, quando Louis-Sébastien Mercier aveva pubblicato la prima edizione di L'an 2440. È comunque verso la fine dell'Ottocento, e per effetto delle influenze della cultura positivista, che la tecnica del viaggio immaginario nel futuro, di solito sotto forma di sogno, diventa un genere diffuso.

107 C. Renouvier, Uchronie (L'utopie dans l'histoire), Paris, Bureau de la Critique Philosophique, 1876; ristampa anastatica Paris, Fayard, 1988. Il sottotitolo completo dell'opera è: Esquisse historique apocryphe du développement de la civilisation européenne tel qu'il n'a pas été, tel qu'il aurait pî être.

${ }^{108}$ Uno dei più autorevoli studiosi del pensiero di Renouvier, Vittore Collina, colloca il nostro personaggio all'esterno del movimento positivista, definendo la sua filosofia un "neocriticismo fenomenistico" di derivazione kantiana, per quanto notevolmente corretta dalle manipolazioni operate dallo stesso Renouvier sulla ricerca kantiana della composizione fra attività del pensiero volta all'universalità e particolarità dei fatti concreti (vedi V. Collina, Plurale filosofico e radicalismo. Saggio sul pensiero politico di Charles Renouvier, Bologna, Clueb, 1980). Certi elementi di fondo della filosofia, in particolare politica, di Renouvier lo pongono in effetti abbastanza al di fuori dell'orizzonte comtiano: basti pensare all'insistenza sulla libertà nelle sue forme individuali e sul senso di una storia che non ha un fine predeterminato, ma si apre agli apporti creativi e non preventivabili degli uomini. Qualche concessione in più rispetto a un presunto positivismo giovanile di Renouvier è rintracciabile in W.M. Simon (Il positivismo europeo nel XIX secolo, cit.), che lo valuta più "scientista" dei neokantiani e sensibile al messaggio di Comte, almeno per quanto riguarda il bisogno di una reazione filosofica all'eclettismo delle accademie di metà Ottocento. Anche Simon riconosce comunque che Renouvier fu divulgatore più di Kant che di Comte, e che in ogni caso non partecipò in alcun modo alla svolta religiosa dell'ultimo Comte. Altro contributo critico recente è quello di G. Cavallari, Charles Renouvier filosofo della liberaldemocrazia, Napoli, Iovene, 1979. Una visione d'insieme delle varie facce del pensiero di Renouvier è deducibile dagli scritti pubblicati intorno al nostro filosofo dall'editore parigino Vrin nel 1927: L. Foucher, La jeunesse de Renouvier et sa première philosophie; G. Milhaud, La philosophie de Charles Renouvier; O. Hamelin, Le système de Renouvier.

${ }^{109}$ Il pensiero filosofico di Renouvier è racchiuso soprattutto nei quattro volumi dell' Essai de critique générale, usciti fra il 1854 e il 1864 e dedicati rispettivamente alla conoscenza, all'uomo, alla natura e alla storia. Nato nel 1815 e morto nel 1903, Renouvier vive un'esistenza dedicata quasi 
tentazioni deterministiche che si potrebbero ricavare dall'impianto scientista del positivismo, per costruire un'immagine del mondo come luogo in cui si esercita al contrario la libertà degli uomini di realizzare responsabilmente le loro aspettative e volontà. Erede più dell'illuminismo che delle filosofie ottocentesche, che anzi accusa di essere orientate a soluzioni autoritarie nel campo del pensiero e in quello dei comportamenti, Renouvier si impegna in una rivalutazione della ragione nelle sue forme individuali. Le influenze del "secolo romantico", e qualche suggestione forse di lontana origine comtiana, si fanno tuttavia sentire nella collocazione del cuore, accanto alla mente, come fattore di miglioramento della condizione umana. Nell'ultimo periodo della sua vita Renouvier approderà poi a una sorta di spiritualismo di intonazione personalistica, recuperando l'immagine deista (e cartesiana) di un Dio creatore che non ha però voluto determinare i destini dell'uomo, lasciandolo libero di scegliere autonomamente il proprio processo di perfezionamento o di decadenza.

Nonostante la sua sostanziale marginalità nei confronti degli aspetti più caratteristici del positivismo comtiano, Renouvier ci interessa in questa sede per un paio di motivi. Intanto egli rappresenta comunque un momento significativo della riscrittura utopica della storia. In secondo luogo le differenti parti, non sempre linearmente collegate, del suo pensiero convergono alla fine intorno a un progetto morale: cioè intorno a uno scopo di ordine analogo a quello dell'ultimo Comte e del suo erede prediletto Laffitte. Pur prendendo ancora un volta più da Kant che da Comte, anche Renouvier propone un'interpretazione della storia sorretta dal riconoscimento di un primato della morale. Tanto che la sua opera forse più significativa, uscita nel 1869, si intitola appunto Science de la morale, e si propone di sistemare razionalmente la morale quasi fosse una disciplina scientifica, per sottrarla alla sua tradizionale sudditanza alle religioni organizzate.

Il discorso morale di Renouvier, di cui va tenuto conto anche in riferimento alla sua opera utopica, fa capo al contrasto dialettico fra "stato di pace" e "stato di guerra". Il primo è contrassegnato dal dominio di una morale razionale pura, e fa quindi parte di una sfera ideale; il secondo è la realtà storica, che è quindi contrassegnata dal conflitto. Questa tende però a espungere progressivamente da sé le tendenze aggressive e a uniformarsi gradualmente all'idea di un incontro pacifico fra gli uomini. La morale agisce nella storia in forme meno pure della totale armonia fra desiderio proprio e rispetto della libertà altrui insita nell'idea perfetta dello stato di pace: il carattere individuale dell'autoconservazione umana rende gli individui gelosi custodi prima di tutto della propria libertà di azione. L'uomo è tuttavia in grado di elaborare nel corso del tempo regole morali "situate", meno perfette di quelle raffigurabili nello stato di pace ideale, ma praticabili nella concretezza dei rapporti storici. Il concetto di morale avanzato da Renouvier, che

esclusivamente alla riflessione e alla divulgazione filosofica, concretizzate, oltre che nei suoi numerosi volumi, nella direzione per tutti gli anni settanta e ottanta della "Critique philosophique". 
come si è detto vuole evitare in ambito etico l'insinuarsi di un ruolo determinante della rivelazione divina, è strettamente legato alla sua visione dell'uomo come essere libero e razionale. Al di fuori di qualsiasi determinismo di tipo evoluzionista, la Science de la morale ribadisce un progetto di ascendenza illuminista, che muove dall'identificazione di sé come essere portatore di ragione, di passione e di volontà, per giungere al riconoscimento dell'altro come fine della propria azione e non come mezzo, sulla scorta ancora di Kant, e di Rousseau. I fattori principali del rafforzarsi della morale nella storia sono il rispetto dei legami razionalmente scelti e il lavoro. Ne deriva un percorso concettuale di carattere morale, ma anche politico, che nasce dal giusto apprezzamento di sé come individuo, continua con la costruzione di un legame contrattualistico fra gli individui sulla base dei valori di eguaglianza e di bene comune, si completa con l'emergere, accanto ai diritti, del dovere della solidarietà sociale. Sono questi i tratti fondamentali del repubblicanesimo di Renouvier, sorretto da considerazioni sociali, disposto a integrare l'impianto liberale individualista con il riconoscimento delle associazioni, e aperto sul piano istituzionale al mantenimento di spazi di democrazia diretta, come correttivi delle alterazioni disugualitarie comunque connesse con l'idea di potere ${ }^{110}$.

E veniamo all'Uchronie, opera intricata che ruota attorno al motivo letterario, ormai consolidato, del finto manoscritto perduto e ritrovato, accompagnato da più appendici comprendenti le notazioni di chi lo avrebbe posseduto nel Sei e nel Settecento, e da una premessa e una conclusione dell'editore, che svela l'interesse e il senso della pubblicazione. La stesura del manoscritto viene fatta risalire all'età dell'Umanesimo, forse opera di un monaco dissidente. Ricomparso a Roma al tempo del rogo di Giordano Bruno, il testo sarebbe poi finito in Olanda, in ambienti umanisti e poi illuministi. Renouvier finge che le note aggiunte al manoscritto, redatte dal suo ultimo conservatore, risalgano ai primi decenni del Settecento.

Punto focale della narrazione storica compresa in Uchronie è la crisi della cultura classica determinata dalla decadenza dell'impero romano, che Renouvier riconduce a motivi di ordine politico e spirituale. Per effetto di un'eccessiva tendenza conquistatrice, che già si era fatta sentire nel mondo greco al tempo di Alessandro, e che ancor più ha segnato le vicende imperiali romane, l'Occidente ha voluto appropriarsi dell'Oriente, finendo però per farsi assorbire dalla sua cultura. Nello schema storiografico idealmente costruito da Renouvier, l'Occidente è il

\footnotetext{
${ }^{110} \mathrm{Nel} 1848$ Renouvier è autore di un diffuso Manuel républicain de l'homme et du citoyen, seguito l'anno successivo da uno scritto sull'Organisation communale et centrale de la République. Queste opere, in particolare il Manuel, gli valgono una lunga fama come scrittore repubblicano, rinforzata dalla sua estraneità alla politica del Secondo Impero. La stima da lui riscossa negli ambienti repubblicani continua perciò fino alla Terza Repubblica, che pure vede progressivamente affievolirsi l'entusiasmo di Renouvier, deluso dal persistente affarismo che si respira negli ambienti del potere. J.A. Scott, autore di Republican Ideas and the Liberal Tradition in France (1870-1914), New York, Columbia University Press, 1951, considera Littré e Renouvier gli autentici capostipiti del pensiero repubblicano nella Francia del secondo Ottocento.
} 
luogo originario dei sentimenti repubblicani, l'Oriente è il mondo della monarchia divinizzata. L'errore, prima del mondo greco, poi di quello romano, è stato quello di lasciar indebolire le istituzioni originarie per seguire le correnti espansionistiche, che hanno prodotto ricchezze squilibrate, corruzione dei costumi fra i potenti, impoverimento relativo delle classi basse, estensione incontrollata della schiavitù. L'espansione a Oriente ha significato l'incontro con culture abituate a un potere autocratico, che nulla aveva a che vedere con la giustizia delle istituzioni repubblicane, le sole che potevano conferire ulteriore durata al mondo classico. Questo assorbimento di culture non proprie ha avuto il suo fulcro, secondo Renouvier, nella commistione fra religione e potere costruita dalle civiltà orientali entrate in contatto con l'Occidente (egizi, babilonesi, persiani), portatrici di superstizione e di celebrazione acritica dell' autorità ${ }^{11}$.

Nell'ambito delle religioni orientali, un elemento di distinzione è comunque costituito dall'ebraismo, su cui l'Uchronie si sofferma a lungo. Piccola ma di grande personalità, la nazione ebraica presenta infatti una tradizione alternativa rispetto alla sacralizzazione del potere concentrato in poche mani. Al di là delle variazioni istituzionali fra un'epoca e l'altra, l'organizzazione sociale degli ebrei è rappresentata da Renouvier come basata sulla piccola proprietà e sulla legge agraria: elementi non molto dissimili dal sottofondo sociale del repubblicanesimo occidentale da lui celebrato. Il monoteismo ebraico, e soprattutto la concezione di un solo Dio immaginato simile all'uomo, se per la sua semplicità rituale costituisce un ostacolo all'evoluzione delle arti, in antico legate a cosmogonie più complesse, per un altro verso segna un progresso morale rispetto al resto dell'Oriente. "Lo spirito fatalista, causa principale del torpore dei popoli, è combattuto con la credenza nell'arbitrarietà divina, prima iniziatrice delle cose, e poi nella libertà degli individui, ai quali una volontà sovrana prescrive leggi non coattive: la coscienza morale si raffina, la giustizia si innalza e tende a divenire indipendente dal dogma, l'abisso della contemplazione panteista si chiude; e la persona cresce, quando prende in considerazione un dio fatto a propria immagine, e un mondo fatto per le persone" 112 .

La possibilità di dialogo fra ebraismo e tradizione filosofica occidentale non trova tuttavia un terreno storico di incontro effettivo, a causa, secondo Renouvier, dell'intransigenza e dell'intolleranza degli ebrei. La stessa chiusura si manifesta

\footnotetext{
${ }^{111}$ Partendo da questa osservazione Renouvier si lascia andare a una lunga digressione sui rapporti fra stato e chiesa, ricondotti a tre modelli principali. Il primo è quello dello stato che ignora le confessioni organizzate, ritenendole un fatto del tutto privato. Il secondo è quello dello stato tollerante, forte perché capace di instaurare una religione civile, ma anche in grado di consentire confessioni particolari, purché rispettose della legge: per Renouvier è questo il modello romano, che conferisce una specie di sacralità laica agli istituti politici senza ammettere una "religione regnante" (Uchronie, p. 54). Il terzo modello, unico presentato come sicuramente negativo, è quello del potere che si identifica con una religione reprimendo le altre: una teocrazia automaticamente contraria alla libertà di coscienza.

${ }^{112}$ C. Renouvier, Uchronie, cit., p. 63.
} 
quando l'erede dell'ebraismo, il cristianesimo, si presenta sulla scena occidentale sotto forma di setta. L'Uchronie non discute la capacità del cristianesimo di parlare alle classi più povere, quelle più deluse dall'ingiustizia sociale della Roma imperiale. Ma il suo limite risiede nella sua inadeguatezza a proporre strategie di miglioramento da realizzare nella storia. Come vedremo, nell'immaginario di Renouvier il cristianesimo primitivo ha il torto di proiettare l'umana ansia di perfezione verso una sfera soprannaturale, e il cristianeismo successivo, quello che si avvia verso il medioevo, ha la responsabilità di accettare o di sollecitare l'abbraccio con poteri autocratici, finendo per essere una variante del dispotismo orientale "appropriata alle idee occidentali" "113, non un'autentica alternativa. La saldatura fra impero romano e cristianesimo, e poi fra monarchie barbariche e chiesa cattolica, costituisce nello schema di Renouvier il passaggio fondamentale per spiegare gran parte dei mali conosciuti dall'Occidente nella sua storia: intolleranza, oppressione, guerra ${ }^{114}$.

Nella narrazione di Renouvier scatta tuttavia a questo punto l'operazione ucronica, tesa a rappresentare il successo originario del cristianesimo in Occidente come un fatto contingente, dovuto come tutti gli eventi storici alla volontà o alla debolezza umana, e non imputabile a un destino inevitabile. La storia di come "avrebbero potuto andare" (ma non sono andate) le cose prende spunto dalla figura di Marco Aurelio, l'imperatore filosofo presentato non come lo stoico rassegnato della tradizione storiografica, ma come un sovrano impegnato a reagire alla decadenza romana. Gli Antonini, di cui Marco Aurelio è la figura di maggior spicco, approntano una risoluta riforma agraria, che combattendo il latifondo e favorendo i piccoli coltivatori rinsalda i legami sociali all'interno dell'impero. Uno di loro, Commodo, riorganizza l'esercito, contrastando con successo le spinte verso l'anarchia militare. La reazione alla crisi delle istituzioni romane toglie fascino e capacità di diffusione alla predicazione cristiana, con l'effetto di circoscrivere la sua influenza ai margini orientali dell'impero, dove si incrocerà più tardi con l'islamismo. Roma si risparmia così il governo di Diocleziano, imperatore adorato alla moda delle superstizioni egizie, e il patto definitivo fra impero e chiesa sottoscritto da Costantino.

\footnotetext{
${ }^{113}$ Ivi, p. 87.

${ }^{114}$ La pregiudiziale anticristiana fa sì che Renouvier eviti accuratamente un luogo comune della storiografia ottocentesca, e cioè la celebrazione del medioevo. Dai rimandi alla storia reale ricavabili dall'Uchronie, esce un'immagine dell'età feudale come contrassegnata dalla dannosa occupazione del potere politico da parte del clero, con tutto quanto ne deriva in termini di sopraffazione della laicità, affievolimento del diritto, diffusione di superstizioni legate al potere (come dimostrerà, alla fine del medioevo, la vendita delle indulgenze). Altra colpa della chiesa medievale è quella di avere, con poche eccezioni, favorito la messa in rovina dell'arte e della cultura antiche. Anche un aspetto in sé non negativo della dottrina cristiana rispetto a religioni più fataliste, e cioè l'idea della libertà dell'anima e del valore delle opere e dei sacramenti, ha avuto sul piano storico un esito discutibile, quando il culto è diventato superstizione dei miracoli, delle reliquie e delle immagini sacre.
} 
Nell'ucronia di Renouvier il cristianesimo, che per il suo carattere monoteista, ereditato dalla religione ebraica, presenta comunque aspetti positivi, tornerà in Occidente molto più tardi, e passando dal mondo tedesco: arriverà insomma sotto la veste del cristianesimo riformato, finalmente capace di dialogare con il patrimonio umanistico della cultura occidentale. Ma troverà allora un ambiente ben diverso da quello su cui aveva potuto far presa nei primi secoli dopo Cristo. Renouvier infatti ha intanto descritto un'evoluzione del mondo romano capace di preservare i propri fondamenti, attraverso una politica di tolleranza, di ricerca dell'armonia sociale fra le classi, di perseguimento della pace. Su quest'ultimo punto, un ruolo decisivo nell'immaginario di Renouvier gioca la ristrutturazione dell'impero, avvenuta in un medioevo ovviamente fittizio, come federazione fra le sue grandi nazioni: la Gallia, l'Italia, la Grecia (e l'idea di una federazione europea costituisce uno dei capisaldi del suo pensiero politico anche per l'attualità). Il contenimento dell'avanzata del cristianesimo (che a un certo punto ha perfino tentato inutilmente una crociata contro l'Occidente) non ha certo eliminato tutti i mali dall'esperienza storica del mondo nato dalla Roma antica: la sua evoluzione, che si concluderà con il completarsi delle moderne tendenze repubblicane, ha richiesto e richiede conflitti a volte intensi, che solo con molta lentezza si possono eliminare dalle vicende umane. Nella rappresentazione di Renouvier la continuità con la cultura classica, effettiva e non solo declamata, avrebbe tuttavia risparmiato una buona dose di lutti. Il suo merito maggiore sarebbe stato quello di impedire l'egemonia confessionale sulle coscienze, perché è proprio dello spirito religioso intransigente approntare inquisizioni e spingere verso la guerra, la cui forma più pericolosa si è dimostrata essere, nella storia, appunto la guerra di religione.

Il punto debole dell'ucronia di Renouvier risiede nel fatto che non si tratta di un divertissement letterario, per il quale oltretutto non sarebbero adatte né la pesantezza dell'impostazione, né la prolissità del linguaggio. Nelle sue intenzioni la descrizione della storia come avrebbe potuto essere sembra costituire invece un tentativo di continuare il discorso filosofico con altri mezzi: ma ciò viene cercato in modo non lineare, spesso contraddittorio, naturalmente scivoloso per l'oggettiva difficoltà di tenere insieme una storia effettivamente accaduta e la trama delle propensioni immaginarie dell'autore. A noi interessa comunque che centro dell'argomentazione, in sintonia con altre parti della produzione di Renouvier, sia un ragionamento intorno alla morale, alle sue potenzialità ideali e agli scacchi subìti dagli uomini nella storia sul terreno etico. Nella rappresentazione dell'autore dell'Uchronie l'influenza che il costume orientale ha esercitato in Occidente, prima dell'avvento del cristianesimo, è sintetizzabile come Antimorale, come sottrazione del potere ad ogni regola. "Agisci in modo che la tua azione possa sempre essere giustificata dalla considerazione di te come centro di tutte le cose" 115 , è la regola dell'azione del principe, intenzionalmente modellata sul rovesciamento

${ }^{115}$ C. Renouvier, Uchronie, cit., p. 69. 
dell'imperativo kantiano. A tutto ciò il cristianesimo ha aggiunto l'Ultramorale, cioè l'ideologia del sacrificio e dell'astensione dai beni di questa terra, per proiettare la perfezione e la salvezza nell'al di là. "All'antimorale, che è l'egoismo dei grandi, eretto a sistema di vita e di pratica, si oppone in Oriente l'ultramorale, che è la dottrina del sacrificio volontario degli umili, in vista della loro salvezza individuale in un' altra vita, o del niente stesso" ${ }^{, 16}$.

Renouvier segue a questo proposito il passaggio da un'antichità modellata sulle virtù civili repubblicane a un imperialismo denso dei segni del governo assoluto, fino all'apparizione nel mondo ebraico, e poi negli ambienti del dissenso romano, del mito del Salvatore che salva il mondo al prezzo di un sacrificio di negazione, a partire dal sacrificio di se stesso. Sul piano storico, tutto ciò ha prodotto il grave inconveniente di deviare la coscienza dall'unica strada di moralizzazione autentica e concreta del mondo, quella avviata dalla filosofia. "Da un lato, la nuova religione può perdere il mondo per salvarlo; predica al mondo la penitenza e il sacrificio, in nome del solo vero Dio; più tardi si sforzerà di sottometterlo e di governarlo, per meglio ottenere con la forza una salvezza che la buona volontà non saprà offrire. D'altro lato, la filosofia si attende il bene degli uomini dalla giustizia e dalla libertà, e avrà per strumento fatale quello che è al contempo il suo principale ostacolo: la politica temporale. Quando questa è lungimirante e saggia, lavorerà con i filosofi all'educazione della ragione pubblica, al miglioramento delle istituzioni e del potere, alla sua propria trasformazione" ${ }^{" 117}$.

La parte polemica dell'argomentazione di Renouvier è dunque volta a denunciare il paradossale intreccio tra rinuncia e intolleranza che proviene dalla morale cristiana, mentre la parte propositiva è intenzionata a ritagliare uno spazio crescente per la filosofia come guida del comportamento umano. Su questo terreno entrano in gioco anche la scienza e la tecnica, che l'Uchronie ricorda come aspetti fondamentali del miglioramento dell'umanità, prodotti, in particolare a partire dalla

\footnotetext{
${ }^{116}$ Ivi, p. 73.

117 Ivi, p. 85. Verso la fine della sua ricostruzione di come le cose avrebbero potuto andare diversamente, Renouvier ribadisce che nella resistenza della filosofia antica di fronte all'avanzata del cristianesimo si trova la chiave di volta per una storia che, nella finzione ucronica, si presenta migliore di quella reale. Senza quella resistenza "forse ancora oggi, dopo mille rivolgimenti, avremmo come consolazione e speranza niente più della morale del sacrificio, del culto di un Dio sofferente e del sogno dell'Assoluto. Ma il trionfo del Bene non sarà dovuto alla dedizione e al sacrificio, parole vane che spesso nascondono i languori e le debolezze dell'anima, o addirittura l'egoismo e l'adorazione di se stessi: il trionfo del Bene verrà dalla Giustizia e dalla Ragione. Non è una teoria ostentatrice e vana dell'Infinito che rafforza la verità a uso delle generazioni future: è la dottrina dell'Armonia, cioè delle relazioni perfette realizzate in un ordine finito. E la salvezza terrestre non ce la apporta una grazia che cade dall'alto, il dono o il merito di uno solo, bensì la catena d'oro degli uomini di retta ragione e di grande cuore, che, di epoca in epoca, sono stati le guide spirituali, i veri redentori dei loro fratelli. In mezzo al mondo essi disegnano il ritratto di un'umanità che si ispira al Bene e della sua azione incessante per sfuggire alle aggregazioni malvagie e per perfezionarsi. Sta a noi fare ciò che essi hanno fatto e collaborare secondo i nostri meriti all'opera di liberazione comune" (ivi, pp. 324-325).
} 
rivoluzione scientifica del Seicento, dalla giusta considerazione per le potenzialità della ragione. Renouvier denuncia tuttavia con lucidità i caratteri ambivalenti del progresso scientifico, che attraverso le sue applicazioni tecniche può essere strumento di vita ma anche di morte. Se certi ritrovati della tecnica sono celebrati nella ricostruzione ucronica di Renouvier (per esempio la bussola e la stampa, ma anche la polvere da sparo, quando serve a difendersi), il vero progresso non sta però nella loro semplice espansione quantitativa, ma nella capacità della coscienza umana di governare le novità e di portarle su un cammino favorevole al miglioramento morale.

La tensione verso il perfezionamento va in definitiva condotta sul solo terreno nel quale può essere davvero vantaggiosa per gli uomini: quello della storia. Anche la storia è, per i moderni, una scienza, ed è bene che sia così; ma Renouvier rincorre in essa non solo una spiegazione dei fenomeni umani, ma anche uno stimolo all'impegno e alla speranza. Nella fittizia introduzione dell'Editore all'Uchronie, Renouvier esplicita le sue intenzioni anzitutto ricollegandosi alla tradizione dell'illuminismo. Il secolo XVIII può essere a buon diritto definito il secolo della storia, nel doppio senso di fiducia dell'uomo di potersi migliorare sulla terra, e di disciplina scientifica che nello studio del passato costruisce una delle condizioni fondamentali affinché l'umanità conosca se stessa e prenda in mano il proprio perfezionamento. Il secolo dei lumi è stato per Renouvier in qualche modo il primo dopo milleottocento anni, perché solo allora "si vide l'umanità prendere se stessa per oggetto, ragionare e lavorare su di sé, contare su di sé" ${ }^{118}$.

Anche nei confronti della razionalità illuministica (e poi positivistica) Renouvier esibisce tuttavia un atteggiamento contraddittorio, di ammirazione per la costruzione dello spirito scientifico e di insofferenza verso le possibili deduzioni deterministiche che da questo si possono ricavare. Il programma dell'illuminismo era legato soprattutto alla necessità di fare della storia una scienza, che come ogni altra scienza si muoveva alla ricerca dei fatti oggettivi e delle leggi della loro concatenazione. Ne risultò uno spirito deterministico che, tranne poche eccezioni (e Renouvier fa in proposito il nome di Condorcet), avrebbe mal sopportato un'opera come l'Uchronie, tesa ad affermare che i fenomeni storici non sono per forza sottratti al controllo della volontà umana. "L'opera storica dell'ultimo secolo si prefiggeva di riformare la storia dei fatti reali, non di immaginare quella dei fatti possibili; di criticare le nostre origini, non di fingerle cambiate; di sostituire le favole ereditate da una chiesa o da una monarchia sacralizzata con il computo esatto e lo spirito positivo degli avvenimenti di cui gli uomini furono autori o vittime; di distruggere l'autorità delle tradizioni fanatiche e superstiziose e, a questo proposito, di professare anzitutto un profondo rispetto per la realtà e un

\footnotetext{
${ }^{118}$ Ivi, p. 12.
} 
inviolabile attaccamento al metodo che la constata con rigore, qualunque essa sia" 119 .

Oltre questa storiografia pure necessaria alla coscienza umana, ma portata inevitabilmente ad appiattire le vicende in un'atmosfera deterministica, l'ucronia insorge per valorizzare la responsabilità degli uomini, singoli e associati, nel volere e nel costruire un mondo diverso da quello ereditato via via da ciascuna generazione. Un'altra parte fittizia del libro di Renouvier, e cioè la postfazione dell'Editore, si incarica di esplicitare il senso dell'operazione specifica racchiusa nell'Uchronie, ma anche di ogni immaginazione a sfondo utopico. Obiettivo del libro, conclude Renouvier, era quello di descrivere una storia "possibile", forse ridimensionata dagli errori che l'autore, senza volere, può averci messo dentro: cosa del resto comprensibile, visto che commettono tanti errori già coloro che scrivono la storia "vera". Lo scopo era in ogni caso quello di diradare l'illusione che, fra tutti gli atti immaginabili, quello compiuto sia sempre l'unico possibile. Con questa notazione Renouvier cerca di dare dignità di senso non solo alla sua ucronia, ma anche alle operazioni utopiche in genere, che appunto rifiutano di vedere i destini dell'umanità ancorati alle condizioni esistenti, dalle quali non si possa derogare. Tutto ciò comporta conseguenze non solo sul modo di scrivere la storia, ma anche sul come ci si atteggia di fronte alla realtà politica e alle aspettative morali. Anche se l'operazione letteraria specifica può essere giudicata poco riuscita, e Renouvier stesso sembra averne coscienza, tuttavia l'autore "avrà costretto lo spirito a fermarsi per un momento attorno al pensiero dei possibili che non si sono realizzati, ed elevarsi così più risolutamente a quello dei possibili ancora sospesi sul mondo. Egli avrà combattuto e, chissà, forse anche scosso i pregiudizi di cui il fatalismo aperto o mascherato è la radice. Avrà prodotto, anche sotto forma di un libro chimerico e difettoso nell'esecuzione, un libro utile" ${ }^{" 120}$.

\subsection{Il futuro come angoscia: a proposito di un'opera "dimenticata" di Jules Verne}

Come si è detto all'inizio, l'ucronia è alla fine dell'Ottocento solo casualmente "utopia del passato", come la concepiva Renouvier. Essa è soprattutto utopia del futuro, e come tale la interpreta l'opera più nota del genere, quel The Time Machine dalla cui citazione siamo partiti per la rassegna dei legami fra scienza, storia e utopia $^{121}$.

\footnotetext{
${ }^{119}$ Ivi, pp. 15-16.

${ }^{120}$ Ivi, p. 470.

${ }^{121}$ Per l'inserimento della dimensione ucronica nel pensiero utopico rinvio alle parti riguardanti la fine dell'Ottocento dei libri di M. Adriani, L'utopia, Roma, Studium, 1961, e di S. Rota Ghibaudi, L'utopia e l'utopismo, cit. Note interessanti intorno agli autori citati in questo capitolo si trovano anche in G. Negley e J.M. Patrick, The Quest for Utopia, New York, Schuman, 1952, e in Utopia rivisitata, a cura di R. Cirio e P. Favari, Milano, "Almanacco Bompiani” 1974.
} 
Il consolidarsi verso la fine dell'Ottocento dell'ucronia volta al futuro come variante della letteratura utopistica, finisce per modificare in profondità anche $\mathrm{i}$ rapporti fra utopia e storia. Finché (dall'Umanesimo al primo Ottocento, con poche eccezioni) la narrazione utopica si era tenuta al di fuori della dimensione storica, disegnando società perfette esterne alle coordinate spaziali e temporali note (isole immaginarie di un tempo immaginario), aveva potuto mantenere fermo il suo progetto pedagogico di fondo. Quel progetto consisteva nell'educare i lettori all'importanza dei valori nella loro interezza, demandando poi agli stesso lettori la responsabilità di riflettere su come e fin dove sia possibile realizzare in concreto i grandi princìpi di solidarietà. Ma l'imporsi delle pratiche storiografiche e delle filosofie storicistiche lungo l'Ottocento non consente più di collocare l'utopia in una dimensione di apparente neutralità rispetto all'effettivo cammino della storia, o di opposizione non detta fra la grandezza ideale delle speranze utopiche e la bassezza delle imperfezioni della realtà. Verso la fine del secolo la descrizione di un futuro immaginato deve per forza fare i conti col progresso storico reale, che è soprattutto segnato dall'espansione dell'attività economica, tecnica e scientifica. La scrittura utopica serve allora a proiettare nel futuro non solo le speranze in un mondo più giusto, ma anche le paure indotte dalle veloci trasformazioni in atto: tanto che la stessa tecnica narrativa (il viaggio immaginario concentrato in un sogno) può essere impiegata sia a fini utopici, sia a fini antiutopici. Una discriminante, che conferisce all'utopismo del futuribile un carattere di profonda ambivalenza nei confronti del progresso scientifico, è costituita dal fatto che la scoperta immaginaria del mondo del futuro cominci talvolta a essere portatrice di un'alternanza fra valutazioni ottimistiche e visioni angosciose.

La traduzione in questa letteratura dell'automatismo del progresso, deducibile dalla filosofia della storia di Comte come dall'evoluzionismo darwiniano, è in effetti fonte da un lato di speranza in un indefinito miglioramento dell'umanità, dall'altro di paura che gli elementi del progresso moderno finiscano per sottrarre agli uomini in carne e ossa (e non all'umanità come concetto astratto) il dominio sulla loro storia. Questa duplicità di atteggiamento nei confronti della tecnologia è presente in termini quasi dichiarati proprio nell'opera di Wells. L'occasione di viaggiare liberamente nel tempo è offerta dal progresso scientifico e tecnico, capace di costruire appunto una "macchina del tempo" che permette a chi la possiede di ergersi potenzialmente al di sopra delle contingenze storiche. E lo stesso progresso si trova inizialmente celebrato all'arrivo del Viaggiatore del Tempo nell'Inghilterra del futuro, quando egli crede che lo sviluppo economico e tecnologico abbia preparato per l'umanità un ambiente di felice perfezione. Ma come sanno bene i lettori di Wells, alle prime impressioni favorevoli segue la progressiva rivelazione di una specie di incubo, l'immagine di un futuro, peraltro 
dato come lontanissimo ${ }^{122}$, nel quale un'organizzazione sociale disugualitaria, rinforzata dalla scienza, ha ormai irrimediabilmente alterato $i$ caratteri dell'umanità, preludendo alla sua scomparsa in quanto tale dalla catena degli esseri viventi. La posizione del fabiano Wells non può essere peraltro catalogata tra $\mathrm{i}$ catastrofismi fine a se stessi. L'indicazione che si può ricavare da The Time Machine, oltre che dalle altre opere distopiche dello scrittore inglese, è la necessità di accompagnare lo sviluppo economico e scientifico con atteggiamenti di ordine morale aperti alla solidarietà fra le classi, con pratiche di riequilibrio sociale e con la conservazione di una tensione umanistica capace di ostacolare l'appiattimento dei singoli e delle masse sulle sole aspettative di ordine materiale.

Può sorprendere che qualche decennio prima di Wells, analoghe preoccupazioni sulla spersonalizzazione inducibile da un mondo tecnocratico corressero per la mente di uno scrittore famoso anzi per le celebrazioni della scienza: Jules Verne. La sorpresa non può però essere assoluta. Da un lato è vero che Verne rappresenta, nel versante della cultura popolare, un atto di fiducia nelle potenzialità della scienza della stessa intensità della filosofia del positivismo. Basti pensare alle opere che negli anni sessanta dell'Ottocento ne costruiscono il successo, dalle

${ }^{122}$ Il Viaggiatore del Tempo approda infatti nell'anno 802.701, facendo la conoscenza prima della classe degli Eloi (la borghesia proprietaria), poi, e in modi avventurosi, di quella dei Morlocchi (il proletariato produttore). Le due classi vivono rigorosamente separate, perché i borghesi sono riusciti a confinare i lavoratori sotto terra, dove sono asserviti alla macchina e al lavoro industriale. Significativa, e forse rispondente a una precisa intenzione critica da parte di Wells, è l'assenza di un ceto medio fra le due classi fondamentali del sistema capitalistico. A una prima impressione gli Eloi sembrano vivere in una condizione di felicità: ma una visione più approfondita della realtà farà scoprire al Viaggiatore del Tempo che si tratta di un ceto di uomini e donne malati nel fisico e tarati moralmente, instupiditi, inutili. Li ha resi così il fatto di non dover né lavorare né combattere in alcun modo per l'esistenza, in quanto pensano che il confinamento del proletariato sotto terra abbia dato loro la sicurezza. Ma essi non si accorgono che con la sicurezza, che si dimostrerà oltretutto non così assoluta come sembra, avanza anche la debolezza conseguente all'assenza di qualsiasi sfida. Quanto ai Morlocchi, il lavoro a cui sono sottoposti e l'ambiente dove sono confinati li sta progressivamente regredendo al rango di animali: ne è una spia eloquente la ricomparsa fra loro di fenomeni di antropofagia. I meccanismi della selezione naturale, trasferiti sul piano sociale senza controlli di ordine politico e morale, stanno insomma rovesciando l'evoluzione nel suo contrario. Il Viaggiatore del Tempo sospetta che "dietro l'angolo" ci possa essere il pericolo di una borghesia "mangiata" in senso proprio, e non metaforico, da un proletariato costretto a condizioni subumane, anticamera della scomparsa dalla terra della stessa umanità in quanto tale. Per un approfondimento su Wells, che qui interessa solo in quanto esponente principale di un genere letterario riscontrabile anche in Francia, rinvio in ogni caso a J.I. Kagarlickij, H. G. Wells: la vita e le opere, Milano, Mursia, 1974; J. Huntington, The logic of fantasy: H. G. Wells and science fiction, New York, Columbia University Press, 1982; M. Draper, H. G. Wells, Basingstoke-London, Macmillan, 1987; F. Porta, La scienza come favola: saggio sui "scientific romances" di $H$. G. Wells, Salerno, Edisud, 1995. Prima di Wells, un'immagine di umanità del futuro privata delle passioni, analoga alla rappresentazione degli Eloi, era stata disegnata da R.H. Hudson nel romanzo A Crystal Age, uscito nel 1887. Il testo di Hudson è peraltro solo uno di quelli che precedono le operazioni utopiche di Wells, come si può vedere nello studio di A. Monti, Dialettica dell'entropia: romanzi utopici prewellsiani, Torino, Giappichelli, 1980; allo stesso autore dobbiamo anche un Invito alla lettura di Herbert George Wells, Milano, Mursia, 1982. 
Cinque settimane in un pallone al Viaggio al centro della Terra, da Dalla Terra alla Luna a Ventimila leghe sotto i mari, tutti romanzi impregnati di previsioni avveniristiche, in particolare in merito ai mezzi adatti ad allargare le frontiere del viaggio possibile all'uomo, che dal suolo vanno trasferendosi sotto i mari, sotto la terra e nell'aria. D'altro lato, le meraviglie scientifiche e tecniche convivono nelle opere di Verne, anche per ragioni letterarie, con un'altrettanto forte capacità di incidere negli avvenimenti da parte di talune personalità eccezionali ${ }^{123}$. Ma quando tale fiducia nell'affermazione dei valori individuali si staglia con meno nettezza, affiora nell'opera di Verne una vena sottile di pessimismo sulle capacità di autentico miglioramento dell'uomo moderno, nonostante tutti i progressi scientifici possibili, che appare in particolare nell'opera postuma L'eterno Adamo.

C'è un caso in cui, nell'opera di Verne, la vena di pessimismo da sottile si fa grossa ed evidente: in un testo rimasto a lungo inedito, riscoperto di recente nella cassaforte di un nipote, troviamo rappresentata in tutta la sua estensione 1' "altra" faccia dello scrittore francese, appunto quella dolente e critica di un progresso basato solo su considerazioni materialistiche e su standardizzati comportamenti di massa. Il titolo dello scritto, che risale al periodo del Secondo Impero, lo stesso dei grandi successi di pubblico di Verne, è Paris au XX siècle, e si allinea così perfettamente alla nostra indagine sulle descrizioni del futuro ${ }^{124}$.

Verne dimostra in questo breve romanzo la sua duplicità nei confronti delle utopie volte al futuro. Da un lato egli coglie le capacità incessanti di innovazione tecnologica presenti nelle attività umane, che possono condurre a una uniformità di condizioni e di comportamenti analoga a quell'equilibrio generalizzato, non più sottoposto a perturbazioni, rincorso appunto dalle utopie. D'altro lato, egli considera una simile situazione non il raggiungimento della felicità, ma anzi una specie di alienazione di massa che, nell'eliminazione delle diversità, affievolisce ogni legame sentimentale autentico, riduce l'influenza del gusto, opprime quelle caratteristiche dell'individualità, come la creazione artistica, che nella storia hanno fatto grande il genere umano ${ }^{125}$.

${ }^{123}$ Per una visione complessiva dell'opera di Verne rinvio a F. Born, Giulio Verne, l'uomo che inventò il futuro, Milano, Mondadori, 1971; B. Becker, Jules Verne: il viaggiatore della fantasia, Milano, Mursia, 1974; J. Chesneaux, Una lettura politica di Jules Verne, Milano, Moizzi, 1976; e soprattutto agli atti del convegno tenuto a Cesena nel 1988 e pubblicati con il titolo Viaggi straordinari attorno a Jules Verne, a cura di F. Pollini e L. Righetti, Milano, Mursia, 1991.

${ }^{124}$ Riscoperto nel 1989, Paris au XX ${ }^{e}$ siècle è stato pubblicato nel 1994 per le edizioni Hachette-Le Cherche Midi, secondo il testo stabilito da P. Gondolo della Riva, autore anche della prefazione. Nella collana "Livre de Poche" di Hachette il romanzo è stato ripubblicato nel 1996. Il volume è stato pubblicato anche in Italia, in un'edizione in lingua originale a fini didattici: J. Verne, Paris au XX siècle, Firenze, Valmartina, 1997. Ne esiste anche una traduzione italiana: J. Verne, Parigi nel XX secolo, Roma, Newton Compton, 1995.

${ }^{125}$ L'ambiguità con cui Verne tratta la materia dell'immaginazione sociale è confermata, a mio avviso, dalla costruzione utopica presente nel suo romanzo 500 milioni della Begum (Milano, Mondadori, 1970), la cui edizione originale risale al 1878. Vi si parla di una città ideale, France-Ville, sorta e sviluppatasi per la libertà lasciata dai suoi costruttori alle iniziative economiche private, e 
Come nelle opere maggiori di Verne, anche in Paris au $X X^{e}$ siècle l'impressione comunicata con più immediatezza è quella di un mondo il cui volto è profondamente cambiato, rispetto a quello a lui noto, per effetto dello sviluppo tecnologico. E come accade spesso nella narrazione utopica, gli elementi dell'immaginazione sono ricavati da una specie di trasfigurazione di dati che partono dall'esperienza concreta dello scrittore, e non da una pura operazione fantastica. Della Parigi del XX secolo scopriamo così in progressione: la rete della ferrovia metropolitana (ispirata a Verne dalle prime costruzioni avviate al suo tempo a Londra) su quattro anelli concentrici, con locomotive tirate da tubi alimentati ad aria compressa; un intenso traffico stradale costituito da specie di automobili, in cui l'apporto della fantasia dello scrittore consiste nel prevedere l'applicazione ai mezzi di trasporto del motore a scoppio sperimentato da Lenoir nel 1859; un sistema di comunicazioni arricchito da un marchingegno simile al nostro fax, anch'esso pensato come estensione di un modello realmente esistente (il "pantelegrafo" ideato dall'italiano Caselli); un grande canale solcato da imbarcazioni trainate da locomotive che transitano sugli argini, canale che, collegandola all'oceano, ha finalmente coronato il sogno di Parigi di diventare una città aperta al mare; e di conseguenza un'enorme struttura portuale, presentata come un sistema complesso di canali, ponti girevoli, magazzini, al quale approdano fra l'altro immensi battelli descritti come città naviganti, con piazze, giardini, tratte ferroviarie interne: il tutto sorvegliato da un faro elettrico alto cinquecento piedi, che può fregiarsi del titolo di "più alto monumento del mondo"126. Quella dell'illuminazione è del resto una delle caratteristiche della Parigi del futuro che Verne vuole trasmetterci più intensamente, anch'essa ampliamento di una tendenza effettiva nelle città dell'Ottocento: a rendere le strade più luminose contribuiscono, oltre all'illuminazione pubblica, le luci proiettate dai grandi magazzini (sorti nell'epoca di Haussmann, la stessa in cui scrive Verne) e dagli alberghi pieni di turisti. La luminosità a ogni ora del giorno e della notte costituisce del resto una

quindi per impulsi di tipo capitalistico. Molto regolamentata, in termini vicini all'immaginario utopico, è invece la struttura architettonica e urbanistica, al fine di raggiungere una condizione di perfezione per quanto riguarda la salute e l'igiene. Minuziose indicazioni sulla costruzione delle case, e altrettanto precise disposizioni sull'ampiezza delle strade, sulla necessaria dotazione di spazi verdi, sulla disponibilità di servizi, sui comportamenti salutari che devono tenere gli abitanti, contribuiscono al raggiungimento dello scopo sottinteso nella fondazione di France-Ville: superare la situazione di invivibilità e di sporcizia che caratterizza le grandi città reali. Verne da un lato comunica ammirazione per quello che la sua fantasia utopica propone. Dall'altro però conferisce a volte una tale radicalità al progetto da farlo sentire una forma di esaltazione irrazionale, come quando scrive che "la pulizia individuale è la principale preoccupazione dei fondatori di France-Ville. Pulire, pulire senza tregua, distruggere appena formati i miasmi che emanano in continuazione da un agglomerato umano, questa è la principale attività del governo centrale": che in effetti opera secondo un'estensione maniacale dei poteri della polizia sanitaria tale da farne un'autorità giusta nei fondamenti quanto pericolosa negli eccessi di perfezionamento, come avviene spesso in Utopia.

${ }^{126}$ J. Verne, Paris, cit., p. 65. Il carattere angosciosamente avvolgente che tutto ciò assume nella descrizione di Verne trova una continuazione nel nome del battello in questione: "Leviathan IV". 
quinta indispensabile per il continuo via vai per le strade di un'umanità che non si meraviglia più delle cose che ha intorno: una notazione che per l'autore di Paris au $X X e$ siècle ha in sé qualcosa di inquietante ${ }^{127}$.

Insieme con tutte queste "meraviglie" il lettore scopre però anche tutto il male del mondo del futuro, che non è solo tecnologico, ma anche governato da un capitalismo monopolistico. Il male, secondo la rappresentazione di uno scrittore non insensibile ai messaggi di giustizia del socialismo, consiste soprattutto nella permanenza e anzi nell'ampliamento, rispetto al presente, delle differenze di classe, espresse anche dai profondi contrasti di ordine urbanistico. Ai "lustrini" di un centro occupato da sedi commerciali, uffici direttivi di imprese e lussuose dimore dei grandi capitalisti, nelle quali essi hanno profuso il loro "cattivo gusto meraviglioso", fanno da contraltare le povere abitazioni popolari delle periferie. A determinare tutto ciò è l'influenza corruttrice di una società costruita e governata in modo univoco, in cui esiste solo il valore del denaro, al quale i parigini hanno imparato a sacrificare sentimenti umani e senso della bellezza. L'intento polemico dello scritto di Verne traspare sin dalla prima pagina, quando il lettore è trasportato al 13 agosto 1960, giorno di distribuzione dei premi agli alunni meritevoli da parte della Société Générale de Crédit Instructionnel. Creata da un barone addetto alle speculazioni finanziarie più di un ventennio prima, al tempo del "regno di Napoleone V", la Société ha trasferito nel campo dell'istruzione la tendenza egemone nel sistema industriale, quella di unire progresso e monopolio. Efficiente, anche per l'essersi affidata a un consiglio di amministrazione composto da soli capitalisti, senza la presenza di un solo sapiente o di un solo professore, la Société ha in effetti acquisito il monopolio completo dell'istruzione in tutta la Francia, usandolo secondo le intime finalità di una specie di capitalismo di stato. "A forza di moltiplicare le succursali dell'Università, i licei, i collegi, le scuole primarie, i convitti della dottrina cristiana, i corsi preparatori, i seminari, le conferenze, gli asili, gli orfanotrofi, una qualche istruzione era penetrata fino negli strati più bassi dell'ordine sociale. Se nessuno leggeva più, almeno tutti sapevano leggere e perfino scrivere; non c'era figlio di un artigiano ambizioso o di un contadino declassato che non pretendesse un posto nell'amministrazione; il funzionariato si sviluppava in tutte le forme possibili, e vedremo più avanti quali legioni di impiegati il governo conducesse al passo, e in modo militaresco" ${ }^{, 28}$.

L'intenzione di Verne è appunto quella di dipingere un futuro in apparenza libero, ma in realtà soggetto a una militarizzazione larvata, sotto il profilo morale peggiore dei vecchi eserciti, perché in essa il valore dell'onore è stato sostituito

\footnotetext{
${ }^{127}$ Scrive Verne che se uno dei nostri vecchi avesse visto le novità della città del futuro, senza dubbio "ne sarebbe stato molto sorpreso; ma gli uomini del 1960 non sostavano più in ammirazione di queste meraviglie; ne profittavano tranquillamente, senza essere più felici perché, dal loro passo forzato, dalla loro andatura frettolosa, dalla loro foga americana, si avvertiva che il demone della ricchezza li spingeva in avanti senza posa né pietà" (ivi, p. 13).

${ }^{128}$ Ivi, p. 4.
} 
dalla subalternità al denaro. Il rimpianto per i valori soldateschi e patriottici del passato, che si affaccia in più di un punto della narrazione di Verne, sembra per la verità un po' fuori posto in un'opera che ha il suo obiettivo principale nella denuncia sociale. Ma anch'esso serve a rimarcare l'appiattimento di ogni tensione vitale, la scomparsa di ogni "eroismo" che caratterizza una società governata dal grande capitale, il cui unico scopo è volto alla produzione materiale. Scopriamo che la Parigi del XX secolo è integralmente mossa da uno scientismo puramente utilitarista dal fatto che praticamente tutti i premi dispensati dalla Società dell' istruzione riguardano appunto le materie tecniche, scientifiche ed economiche. L'unica eccezione è costituita dallo studente premiato per ultimo, Michel Dufrénoy, che si è distinto, fra un serpeggiante biasimo generale, per la capacità di comporre poesie in latino. Logico che il premio ricevuto, il "Manuale del buon direttore d'officina", venga subito gettato via dal vincitore. La prosecuzione del racconto si affida alla descrizione del contrasto fra i costumi economici imperanti e la disperata ricerca di una continuità con la cultura della tradizione umanistica. Da un lato sta il grande mondo dei valori materiali, gretto, di un cattivo gusto e di un egoismo davvero "ideali", in cui qualche figura di tecnocrate capace di interpretare qualsiasi cosa solo attraverso parole e immagini tratte dalla scienza e dall'economia capitalistica, viene satiricamente presentato come il "prodotto naturale di questo secolo industriale" ${ }^{\text {"29 }}$. Dall'altro sta il piccolo gruppo degli irriducibili fautori della cultura umanistica e della creazione artistica: una specie di

\footnotetext{
${ }^{129}$ Ivi, p. 21. La satira di un mondo tutto votato all'espansione economica spinge qualche volta Verne a ironizzare anche sulle macchine, che pure sono una delle sue passioni letterarie. Accade per esempio che il protagonista, alla inutile ricerca di un posto di lavoro per lui adatto, sia temporaneamente assunto da una banca, e piazzato a controllare una grande macchina calcolatrice simile a un enorme pianoforte. "Spingendone i tasti, si ottenevano all'istante totali, resti, prodotti, quozienti, regole di proporzionalità, calcoli di ammortizzamenti e di interessi composti per periodi infiniti e a tutti i tassi possibili. Aveva note alte fino al centocinquanta per cento! Niente è meraviglioso come queste macchine ..." (ivi, p. 35). L'intonazione parodistica contro le macchine che serpeggia qua e là nel testo di Verne ricorda quella, più insistita e approfondita, presente in Erewhon di Samuel Butler. Uscito nel 1872, Erewhon (evidente rovesciamento di Nowhere, il "nessun luogo" delle utopie) rincorre un effetto satirico presentando un mondo immaginario in molte parti rovesciato rispetto alle abitudini conosciute. Così la scienza a Erehwon non si basa sui dati reali, ma su un fantasioso calcolo ipotetico; i malati non sono curati, ma puniti per la loro imprevidenza, al pari delle vittime delle truffe; i neonati, attraverso la firma di un tutore, liberano i genitori dalla responsabilità di averli messi al mondo; e appunto le macchine sono messe fuorilegge, per evitare che, riproducendosi, emarginino l'uomo dal progresso e lo riducano a proprio servitore. I passaggi satirici di Butler non hanno uno scopo puramente corrosivo, ma pedagogico: gli elementi del vivere reale, rapportati per confronto a un immaginario rovesciato, svelano un bisogno di correzione ragionevole che non sarebbe avvertibile se ci adagiassimo nell'accettazione acritica di tutto ciò che esiste. Così, una soluzione praticabile del problema del macchinismo, e della ricerca scientifica che ne sta alla base, non è quella di mettere per davvero le macchine fuorilegge. Ma non è nemmeno quella di sopportare il loro inserimento nella vita associata senza ravvisarne i pericoli, e quindi senza sottoporle a filtri di ordine sociale e morale. Su Butler rinvio comunque a L.E. Holt, Samuel Butler, Boston, Twayne, 1964; I. Roventi, Utopia e tecnologia in Samuel Butler, in Estetica e società tecnologica, Bologna, Il Mulino, 1976; P. Raby, Samuel Butler: a biography, London, Hogarth, 1991.
} 
bohème composta dallo studente-poeta che abbiamo appena incontrato, da due suoi amici l'uno musicista e l'altro pittore, da uno zio bibliotecario, da un vecchio professore di retorica e dalla sua giovane e bella nipote (di cui naturalmente Michel si innamora ...).

Tutti questi personaggi costituiscono ovviamente una presenza critica nei confronti del mondo dominante e dei suoi fondamenti ideali: ma sono destinati invariabilmente a soccombere di fronte a un potere più forte di loro. I giovani, che aspirano a un'arte autentica e disinteressata, votata esclusivamente al bello, si dimostrano inetti di fronte a qualsiasi altra occupazione, perché sono incapaci di inserirsi in un lavoro che richiede esecuzione passiva e non intelligenza creativa ${ }^{130}$. Quando qualcuno di loro riesce a entrare in qualche istituzione artistica, ne viene presto allontanato, perché si rivela non disposto a piegarsi all'uso strumentale, di celebrazione del potere capitalistico, assunto da tutte le forme di comunicazione culturale, anche da quelle pubbliche. Il bibliotecario vede ridursi il proprio lavoro a una specie di sopravvivenza catacombale, in una società in cui i romanzi dei grandi scrittori ottocenteschi (Verne cita più volte Hugo) sono ormai introvabili, rimpiazzati da una miriade di manuali tecnici e scientifici. L'unico volume davvero celebrato è l'avveniristico "Grande Libro", un marchingegno con pagine larghe tre metri nel quale vengono incise le operazioni giornaliere della banca più importante di Parigi (in sintonia con la definizione di "tempio dei templi" riservata da Verne alla Borsa). Quanto al professore, il suo destino è di perdere il posto per mancanza di allievi, perché nessuno in un mondo siffatto vuole più studiare retorica.

La vicenda, che Verne non volle pubblicare probabilmente per il suo carattere troppo antitetico rispetto all'immagine di sé costruita presso il grande pubblico, ha il suo compimento nel brusco fallimento delle illusioni d'amore e di creazione in un mondo estraneo ai sentimenti. Michel, che si reca dal professore per dichiarare finalmente e in modo impegnativo il proprio amore per la nipote, trova la casa

\footnotetext{
${ }^{130}$ Degna di interesse è fra l'altro un'invettiva contro la proprietà privata. A un certo punto della narrazione il problema principale di Michel (quello di non riuscire a mantenere alcuna occupazione, perché non integrato nell'ideologia produttivistica che è diventata l'unico sentire del mondo) viene scherzosamente analizzato dai suoi amici con un linguaggio pseudoscientifico: "Dato un ragazzo che non può essere né un finanziere, né un commerciante, né un industriale, come può egli trarsi d'impaccio in questo mondo?". Qualcuno evoca ridendo la sola quarta possibilità esistente: essere proprietari. E ne riceve, da parte di un amico di Michel, una grottesca difesa della proprietà, così volutamente enfatica da costituirne in realtà un atto di condanna: "Quando si pensa che un uomo, un tuo simile fatto di carne e ossa, nato da una donna, da una semplice mortale, possiede una determinata porzione del globo! che questa porzione del globo gli appartiene a titolo personale, come la testa, e spesso ancora di più! che nessuno, nemmeno Dio, può togliergli questa porzione di globo, che trasmette ai suoi eredi! che egli ha diritto di scavare, di rivoltare, di edificare a proprio piacere la sua porzione di globo! che l'aria che la circonda e l'acqua che la bagna, tutto è suo! che egli può bruciare il suo albero, bere i suoi ruscelli e mangiare la sua erba, se gli piace! che ogni giorno egli si ripete di avere una parte sua nella terra che Dio ha creato il primo giorno del mondo, e si dice che quella superficie di emisfero è ben sua, con le seimila tese di aria respirabile che si innalzano al di sopra e il migliaio e mezzo di leghe di scorza terrestre che si spingono al di sotto!" (ivi, pp. 78-79).
} 
deserta, perché i due sono stati sfrattati e cacciati da Parigi. In preda alla disperazione vaga alla cieca per una notte intera per le strade del centro e della periferia di Parigi, avendo occasione di imprecare contro tutte le immagini luminose di cui si è detto in precedenza, che gli sembrano la patina di monumentalità posta sopra una società assurda. La peregrinazione del giovane studente si conclude al cimitero del Père-Lachaise, un luogo caro alla sensibilità romantica ma, se vogliamo, anche alla religione dell'umanità derivata dal positivismo. E lì, nell'unico luogo in cui ancora si può coltivare la memoria, egli si accascia, vicino alla tomba di de Musset: simbolo conclusivo di una vicenda che vuole appunto rappresentare la morte dell'arte nel mondo della tecnica e degli affari.

\subsection{Il nuovo mondo dopo la catastrofe: Tarde e la storia futura}

Nel 1896 la "Revue internationale de sociologie" ospita uno dei testi che più creativamente riprendono lo schema della descrizione utopica del futuro: il Fragment d'histoire future di Gabriel Tarde. Quando esce il racconto, il suo autore è già noto alle cronache scientifiche e culturali francesi, prima per gli scritti di criminologia, poi per il passaggio alle argomentazioni di ordine sociologico ${ }^{131}$. Egli ha elaborato il Fragment, la cui prima stesura risale agli anni ottanta, insieme con un'opera come Les lois de l'imitation, pubblicata nel 1890: l'opera, cioè, che unita alla successiva Logique sociale (del 1894), costituisce il corpo principale dell' argomentazione sociologica, venata di rimandi psicologici, di Gabriel Tarde ${ }^{132}$.

Le Lois de l'imitation, delle cui idee il Fragment è una specie di trasferimento avventuroso al piano utopico, sono un esempio di quell'intensa quanto variegata ricerca delle leggi scientifiche che sottostanno alla socialità fra gli uomini, con cui si va formando la sociologia. Scaturite dalla cultura del positivismo, opere come

\footnotetext{
${ }^{131}$ Vissuto fra il 1843 e il 1904, Gabriel Tarde risiede a lungo nel Périgord, dove svolge l'ufficio di giudice istruttore. A questa esperienza sono legati i suoi scritti di criminologia, soprattutto la Criminalité comparée, del 1886, che corregge in parte il punto di vista di Lombroso, pur all'interno di un'ammirazione per l'opera meritoria svolta dal criminologo italiano. Per Tarde l'origine del crimine non può essere fatta risalire a sole ragioni fisiologiche, ma vanno tenuti in considerazione anche $\mathrm{i}$ fattori di ordine sociale e culturale, significativi nel far passare o no all'azione delittuosa il temperamento criminale. Verso la fine degli anni ottanta i suoi interessi in campo giudiziario, di cui è ulteriore testimonianza la Philosophie pénale (del 1890), cominciano a saldarsi con osservazioni di sociologia generale. Nel 1894 Tarde si trasferisce a Parigi, chiamato dal ministero della giustizia, e nella capitale può completare l'opera di ricognizione sociologica intrapresa nell'ambiente appartato del Périgord.

${ }^{132}$ Le maggiori opere sociologiche di Tarde sono disponibili anche in italiano: G. Tarde, Scritti sociologici, a cura di F. Ferrarotti, Torino, Utet, 1976. Per una visione d'insieme del nostro autore rinvio, oltre all'introduzione di Ferrarotti all'edizione appena citata, all'opera di J. Milet, Gabriel Tarde et la philosophie de l'histoire, Paris, Vrin, 1970, e ai due volumi pubblicati di recente da R. Bisi: Credenze, desideri e imitazione: un approccio al sistema socio-psicologico di Gabriel Tarde, Bologna, Clueb, 2000; e Gabriel Tarde e la questione criminale, Milano, Angeli, 2001.
} 
quella di Tarde costituiscono per la verità una fase di transizione, sospese come sono fra osservazione empirica e ideologia; in esse si cominciano tuttavia a notare i presupposti dell'emergere della sociologia come scienza (e non come filosofia, quale era in fondo in Comte). Anche Tarde, al pari di Comte, è comunque votato alla ricerca di una linea unitaria attorno alla quale si possa dare uniformità e senso all'indagine sociale, e pensa di trovarla nel modello della mente creatrice, che è la caratteristica peculiare della specie umana. L'uomo modello crea perché spinto dal desiderio di innovazione. Questo desiderio non è tuttavia qualcosa di puramente soggettivo; esso nasce dalle credenze e dai bisogni di una determinata epoca, che le personalità eccezionali sanno interpretare e volgere a fini evolutivi in virtù della loro superiorità mentale, che è anche il prodotto della loro cultura. Il desiderio di innovazione produce un'invenzione storicamente situabile, la quale si propaga nel corpo sociale attraverso il meccanismo psicologico dell'imitazione, che per Tarde costituisce la vera molla del progresso sociale, e quindi anche la base per ogni sociologia.

Come si è potuto capire dal riferimento alle personalità superiori, un carattere importante nell'argomentazione di Tarde assume la convinzione della disuguaglianza naturale fra gli individui, già manifestatasi, prima di lui, in seno al positivismo. L'invenzione non proviene infatti in misura analoga da tutti gli uomini, ma deriva dagli "attori" psichicamente più forti, in grado di influenzare la massa dei loro simili. In sintonia con umori presenti in larghe fasce delle nascenti scienze umane, in Francia e fuori, Tarde interpreta l'invenzione come inevitabilmente incorporata in un'élite: in casi particolari può addirittura manifestarsi in pochissime personalità eccezionali, che possono legittimamente aspirare al ruolo di capi. Gli esseri viventi, in quanto non uguali, possono però essere diversi anche negli atteggiamenti. Di fronte all'invenzione che una determinata élite propone all'interno di una comunità, mentre una parte della massa si fa sedurre dall'imitazione altri soggetti, con doti psichiche di maggiore resistenza, evidenziano al contrario sentimenti di opposizione. Tutta la vita individuale e associativa è del resto contrassegnata da dualismi: sia sul piano dei sentimenti e delle sensibilità (amore-odio, piacere-dolore), sia su quello dei ritmi sociali, che si presentano sempre sotto forma di polarità antitetiche: conservazioneprogresso, pace-guerra, ricchezza-povertà, domanda-offerta. La radicalità delle opposizioni non può tuttavia durare indefinitamente, pena l'impossibilità di qualsiasi ordine sociale. L'arco dell'argomentazione di Tarde, partita da un'enfatica sottolineatura del peso sociologico dell'invenzione, si chiude così attorno al concetto di adattamento, definito come una spinta incessante, ma mai integralmente soddisfatta, all'equilibrio fra imitazione e opposizione.

L'opera utopica, il Fragment da cui siamo partiti, è un po' un gioco letterario, un po' una continuazione delle idee sociali del suo autore sotto forma di immaginazione, volta fra l'altro a delineare un ipotetico raggiungimento di un 
equilibrio forse finalmente stabile, come è ricorrente nel pensiero utopistico ${ }^{133}$. Prima di arrivare alla presentazione di tale mondo rinnovato, Tarde mette tuttavia in scena l'espediente narrativo di una catastrofe naturale, che costituisce una specie di occasione per l'umanità di lasciarsi alle spalle comportamenti ormai superati o inutili, per sperimentare la propria vita su basi concettuali e organizzative differenti ${ }^{134}$.

La struttura letteraria dell'opera è abbastanza complessa, riconducibile a una narrazione in tre tempi. Dapprima, nella finzione, l'anonimo autore del Fragment ricostruisce lo sviluppo che il nostro mondo è destinato a conoscere in un futuro prossimo. Poi ci racconta che, verso la fine del XXV secolo dell' "era preistorica" (cioè dell'era cristiana), una catastrofe inabissa la civiltà esistente, ma non la distrugge del tutto: un piccolo gruppo di uomini e donne riescono a sopravvivere e a porre le basi per una nuova civiltà. La terza fase è quella del consolidamento di questo nuovo mondo, narrato in termini di adesione ai canoni della letteratura utopistica nel Fragment, che si figura come scritto nell'anno 596 dell'"era della Salvezza", il nome che gli uomini del futuro remoto hanno dato alla loro epoca. Fulcro della narrazione è comunque il momento della catastrofe, dal quale si guarda prima all'indietro, verso la nostra epoca, e poi in avanti, nell'utopia definitiva del futuro più lontano.

Nella prima parte del suo racconto Tarde, collocandosi appunto verso la fine del XXV secolo, ipotizza che sia avvenuta un'evoluzione del nostro mondo nel segno della prosperità. All'inizio dello stesso secolo gli uomini sono finalmente riusciti a dare stabilità al progetto di una federazione asiatico-americano-europea in grado di controllare tutto il mondo, che ha concluso un lungo tempo di guerre. L'epoca

${ }^{133}$ Del breve scritto esistono varie traduzioni italiane: fra queste G. Tarde, Frammento di storia futura, a cura di V. Petrucci, Napoli, Esi, 1991. Nella Postfazione Petrucci apparenta il Frammento, più che alle contemporanee utopie ambientate nel futuro, al genere dell'apologo fantasioso e satirico in voga nel secolo dei lumi, citando l'episodio dei Trogloditi nelle Lettere persiane di Montesquieu, la Favola delle api di Mandeville, Micromégas di Voltaire. Quanto all'ucronia, i precedenti indicati sono naturalmente quelli di Mercier e di Renouvier. Interessante è la consideraziorne effettuata da M. Donzelli ("Fragments" dal lessico filosofico di Gabriel Tarde, in Figure dell'individualità nella Francia tra Otto e Novecento, a cura di M. Donzelli e M. Fimiani, Genova, Marietti, 1993, p. 301), a proposito della collocazione del Fragment nel genere utopico-ucronico: "Il Fragment ci presenta un paese immaginario dove un ideale governo regna su un popolo felice: le caratteristiche dell'utopia sono dunque presenti. Ma l'utopia di Tarde non è e non vuol essere profetica, essa è uno strumento della speculazione teorica che si incontra felicemente con una tendenza immaginativo-letteraria".

${ }^{134}$ La fine violenta della società conosciuta, presupposto per poter dar luogo a un mondo alternativo, è un espediente letterario usato anche da altri scrittori del tempo di Tarde. È per esempio presente, ma con intenzioni distopiche, in After London di Richard Jefferies, del 1885, in cui l'umanità è rappresentata regredita a una specie di medioevo, a causa di un misterioso cataclisma. Ancora più frequente è l'eventualità che la catastrofe, da cui è destinata a partire una nuova storia dell'umanità, sia prodotta dalla volontà umana sotto forma di una rivoluzione: il caso più noto, su cui torneremo, è quello di Morris, ma è uno schema presente anche nel romanzo Caesar's Column. A Story of the Twentieth Century, pubblicato nel 1892 da Edmund Boisgilbert, pseudonimo di Ignatius Donnelly, in cui si narra di una rivoluzione violenta che ha eliminato i grandi monopoli, a partire da quelli ebraici. 
militare non aveva peraltro solo conseguenze negative: essendo divenuto costume di tutti gli eserciti scartare dalla leva i soggetti belli e prestanti, e inviare al fronte, per essere eliminati, i più brutti e disgraziati, alla fine dell'era preistorica la guerra aveva contribuito al miglioramento fisico ed estetico della specie ${ }^{135}$. Nella federazione si parla una sola lingua universale, il greco, che in precedenza era usata soltanto nell'impero elleno-russo: l'unificazione linguistica su scala planetaria permette un'immediata circolazione mondiale dei prodotti, in uno scenario ipotetico che oggi definiremmo di tipo globalizzato. Economia e tecnologia (soprattutto in campo elettrico) hanno contribuito alla formazione di una civiltà prospera: prima della catastrofe la produzione si svolge in grandi falansteri internazionali, per far funzionare i quali sono sufficienti tre ore di lavoro a testa.

Tarde si mette insomma, in queste previsioni, nell'ottica delle speranze espresse dalla letteratura utopistica progressiva del suo secolo, di ascendenza socialista come di derivazione positivista. La scienza, che ha il merito di aver consentito tutto ciò, non è peraltro celebrata dai nostri discendenti ideali con la stessa nostra intensità, soprattutto perché ha perso molti dei caratteri "eroici" delle origini. "Semplificata e perfezionata al massimo, cristallizzata in schemi rigidi e immutabili, capace ormai solo di progredire impercettibilmente, essa era tenuta in ben scarsa considerazione, niente più che un surrogato del catechismo d'una volta" ${ }^{136}$. La mancanza di stimoli verso azioni eccezionali è l'unico difetto interiore di questa società, basata sulle aspettative materialistiche di una mediocre borghesia che a suo modo ha ragione di credere - come annota Tarde - che l'inventore della sociologia non sia stato Comte, ma Menenio Agrippa, col suo apologo volto a identificare lo stomaco come organo fondamentale dell'esperienza sociale. Ma la sconfitta della miseria è comunque un risultato positivo, anche se ha per effetto quello di modificare il desiderio umano, in particolare quello delle personalità superiori, che come sappiamo giocano un ruolo essenziale nella visione sociologica di Tarde. Tramontato l'impulso economico-scientifico, ridottosi quello erotico (perché in un mondo di tutti belli anche la conquista amorosa si banalizza), l'ambizione umana si è spostata sull'occupazione del potere politico. In un mondo divenuto un unico stato con miliardi di cittadini, non occorre peraltro diventare capo supremo per sentire soddisfatta la propria sete di potere: un semplice deputato, rappresentando dieci milioni di elettori, è già divenuto una specie di feudatario. Squilibri politici a favore di un'élite ristretta e una certa qual monotonia

\footnotetext{
${ }^{135}$ Come vedremo, pulsioni di tipo eugenetico si affacciano anche in altri punti della narrazione di Tarde. Presente tradizionalmente nella letteratura utopica, il tema della riproduzione controllata a fini di miglioramento della specie ritorna anche in altre utopie del tempo di Tarde: per esempio nell'Anno 3000 di Paolo Mantegazza, del 1897, in cui vengono eliminati i bambini ammalati e i delinquenti per ragioni ereditarie (sulla scia, ovviamente, delle teorie di Lombroso); e nella Lettre de Malaisie pubblicata nel 1898 da Paul Adam, in cui si narra di una nuova umanità insediata nel Borneo, la cui vita associata è caratterizzata dalla separazione fra i giovani, sani e belli, e i vecchi, malati e brutti.

${ }^{136}$ G. Tarde, Frammento, cit., p. 15.
} 
di fondo non riescono tuttavia a scuotere la convinzione degli abitanti del XXV secolo di vivere in una felicità, almeno materiale, senza precedenti ${ }^{137}$.

A un certo punto irrompe però la catastrofe. Dopo aver già dato segni di affievolimento, a partire dall'inverno del 2489 il calore solare quasi non giunge più sulla terra: la conseguente, terribile glaciazione produce in pochi anni miliardi di morti. A salvare quel che resta dell'umanità è uno dei pochissimi viaggiatori rimasti (perché sulla terra non c'è quasi più nulla da scoprire), dal nome allusivo di Milziade, dotato di una qualità ormai quasi perduta fra i cittadini del futuro, quella del coraggio. Milziade raduna attorno a sé le poche centinaia di uomini e donne sopravvissuti (che sono fisicamente i più forti, perché sono stati capaci di resistere al freddo) e indica l'unica strada per la salvezza: entrare nelle viscere della terra ${ }^{138}$. La garanzia di prosecuzione dell'umanità del futuro è quindi il ritorno a una specie di società troglodita, che progressivamente saprà costruire un nuovo mondo scavando nelle rocce. Tutto ciò non costituisce però un ritorno allo stato selvaggio: gli uomini successivi alla catastrofe sanno sopravvivere e tornare a prosperare perché, pressati dal bisogno, sono capaci di ricondurre al centro della loro attività la creazione scientifica. Così, per alimentarsi, la chimica insegna agli uomini del futuro a sfruttare a tal fine anche le rocce; i bisogni energetici sono soddisfatti con lo sfruttamento del calore interno alla terra; nuovi macchinari consentono a tali uomini di risalire periodicamente in superficie, dove sotto il ghiaccio si trovano le carcasse commestibili degli animali uccisi dalla glaciazione. Il loro capo, che salva l'umanità con la propria capacità di porre energia ed eloquenza al servizio dell'immaginazione, ha un'idea decisiva quando impone di portare sottoterra la memoria della cultura umana: una copia di tutti i libri importanti, i modelli di ciascuno stile architettonico, i dipinti più belli e così via, nella convinzione che la vita continuerà se sarà salvato "il vero capitale umano, l'eredità spirituale del passato" ${ }^{\prime 39}$. La salvezza dell'umanità proviene in definitiva da molti degli elementi

\footnotetext{
137 'L'universo respirava. Un po' sbadigliava, è vero, ma, per la prima volta, sbocciava nella pienezza della pace, nell'abbondanza quasi gratuita di ogni bene e nella più brillante fioritura - o piuttosto, ostentazione - di arte, di poesia, ma soprattutto di lusso, che la terra abbia mai conosciuto" (ivi, p. $18)$.

${ }^{138}$ In calce alla prima edizione del Fragment, Tarde afferma di essersi ispirato, per l'idea della continuazione della vita sottoterra, a un passo di Berthelot riportato nel Journal dei Goncourt, in cui il grande chimico affermava che l'eventuale raffreddamento del suolo avrebbe costretto la civiltà a discendere sotto la crosta terrestre.

${ }^{139}$ G. Tarde, Frammento, cit., p. 37. Oltre ai fattori culturali e imitativi propri della sociologia di Tarde, nella costruzione del mondo sotterraneo gioca all'inizio un ruolo rilevante anche lo spirito di sacrificio individuale, qui recuperato positivamente, a differenza, per esempio, che nella morale di Renouvier. Una incrinatura nella fiducia sociologica di Tarde sulla centralità dei fattori culturali nella modellazione delle comunità, risiede nel fatto che questo sentimento di sacrificio è descritto come forza più biologica che acquisita: "E, per ammirevole che sia stato, nell'epoca di cui parlo, questo bisogno collettivo di sacrificio individuale, c'è forse da stupirsene, quando si sa, dai trattati di storia naturale, che dei semplici insetti, dando lo stesso esempio di abnegazione lungimirante, spendevano le loro ultime forze, prima di morire, ad ammassare provviste inutili per loro stessi, utili solo nell'avvenire, alla loro larva nascente?" (ivi, p. 36).
} 
indicati da Tarde come componenti fondamentali della ricerca nei suoi trattati sociologici: l'invenzione da parte di individui di qualità superiore, l'imitazione diffusa su scala sociale, la presenza di un apparato di conoscenze e di convinzioni condivise.

Passata la fase avventurosa dell'occupazione del mondo sotterraneo da parte dei sopravvissuti, superata anche l'inevitabile crisi di assestamento sul piano politico dovuta alla morte di Milziade, alla fine gli uomini costruiscono una società perfetta. La vera utopia di Tarde si rivela essere questa, e non quella del periodo precedente la scomparsa del calore solare. Il perfezionamento dell' "era preistorica" non era infatti un fenomeno del tutto positivo, perché aveva come corollario l'inconveniente di snervare l'uomo nelle mollezze di una soddisfazione esclusivamente materiale. Solo dopo la catastrofe si apre la definitiva età della Salvezza, i cui elementi possono essere sintetizzati nella prevalenza dei caratteri antinaturalistici, estetici ed elitari.

L'antinaturalismo è dato dal fatto che gli uomini del futuro remoto hanno scelto di non dividere il loro nuovo mondo con nessun essere vivente al di fuori di loro stessi. Nell' "arca" ideale costruita sotto terra Milziade ha voluto tener fuori piante e animali, in quanto elementi di distrazione dell'umanità da se stessa. In questo modo gli uomini risultano in relazione fra loro solo per effetto del legame sociale. La società degli abitatori del sottosuolo si configura come un "esperimento prolungato di sociologia" ${ }^{140}$, in cui l'uomo può ricavare sostentamento e piacere solo dai propri simili, a parte le risorse alimentari derivate tuttavia da una natura ormai solo inanimata.

Le difficoltà della ricostruzione e del modellamento di un mondo sempre più vasto (come Tarde lo raffigura) scavato nella roccia, fanno sì che i piaceri materiali siano meno perseguiti rispetto alla tradizione storica. In un ambiente del genere gli alti sforzi operativi comunque richiesti per ogni attività produttiva, per quanto aiutata dalle conoscenze scientifiche vecchie e nuove, conducono a una inevitabile semplificazione dei bisogni. Le necessità alimentari sono uniformi, il vestiario ridotto all'essenziale dal fatto di vivere a temperatura costante e dalla mancanza del bisogno di nascondere corpi tutti belli, le abitazioni sono costrette dalla conformazione dell'ambiente a essere strutture cellulari. Le pulsioni economiche si sono perciò attenuate: la riduzione dei bisogni materiali comporta un minimo di lavoro utilitario, e con ciò il superamento di rapporti sociali obsoleti e un tempo conflittuali, come quelli tra produttore e consumatore e tra imprenditore e

\footnotetext{
${ }^{140}$ Ivi, p. 43. Nella sua polemica antinaturalistica Tarde esprime il distacco da interpretazioni sociologiche troppo ancorate al dato naturale, oltre che sue idiosincrasie personali, come accade spesso agli scrittori di utopie. Parlando successivamente della costruzione di città sotterranee, Tarde riprenderà la provocazione in questi termini: "L'uomo, inurbandosi, si è incivilito. Da quando tutte le specie di alberi e di bestie, di fiori e di insetti, non si interpongono più tra gli uomini, da quando tutte le varietà di bisogni grossolani non ostacolano più lo sviluppo delle facoltà umane, sembra che tutti nascano educati e civili, come tutti nascono scultori o musicisti, filosofi o poeti, parlando la lingua più corretta con l'accento più puro" (ivi, p. 54).
} 
lavoratore. Il mondo di domani ha scoperto che la società non consiste essenzialmente in uno scambio di servizi, bensì di riflessi volti al godimento estetico (fondamento di ogni autentica civiltà secondo Tarde). Riprendendo con altre parole la sua convinzione sul ruolo sociale dell'imitazione, il nostro sociologo scrive nel Fragment: "Scimmiottarsi reciprocamente, e, a forza di scimmiottarsi, differentemente combinati, giungere all'originalità: ecco l'essenziale" ${ }^{\prime 41}$.

In questo modo sembra a Tarde di poter correggere l'errore del socialismo, che non è tanto quello di rincorrere una intensa vita in comune, bensì di pensare tale vita in comune come soddisfazione anzitutto di rivendicazioni materiali, anziché estetiche. I "neotrogloditi", come potremmo definire i suoi eroi, sono riusciti nel compito di costruire un mondo perfetto perché, nella scala della considerazione sociale, hanno sostituito il capitalista con l'artista: questo produce per passione, quello per uno spirito di tornaconto antisociale. "Divertirsi (o soddisfarsi) ciascuno separatamente, e servirsi a vicenda, era il vecchio ideale sociale, a cui abbiamo sostituito questo: servirsi da sé e affascinarsi reciprocamente. La società, dunque, non riposa più sullo scambio di servizi, ma sullo scambio delle ammirazioni o delle critiche, dei giudizi favorevoli o severi. Al regime anarchico delle bramosie è succeduto il governo autocratico dell'opinione, diventato onnipotente" ${ }^{\text {"142 }}$.

Sulla base di questo criterio Tarde, secondo una tecnica consolidata nella letteratura utopica, prolunga il suo discorso nella descrizione di vari aspetti specifici della vita associata degli uomini del futuro, nel campo dell'organizzazione urbanistica, dei costumi, della conformazione del potere. Sappiamo così che, nonostante le ovvie difficoltà della modellazione dello spazio, quegli uomini vivono in città con strade spaziose e ben illuminate, dotate di musei e biblioteche, con un'attività giudiziaria che va scomparendo (naturalmente perché non ce n'è bisogno) e strutture politiche molto semplici. La società nel suo complesso è articolata in città, in ciascuna delle quali vive una specifica aggregazione di coloro che operano nello stesso settore di produzione di godimento estetico o di soddisfazione dei pochi bisogni materiali rimasti. Abbiamo così una città di pittori, una di scultori, una di musicisti e così via, fino agli esponenti delle varie branche scientifiche. Tarde si concede in proposito il vezzo di escludere l'esistenza di una città dei filosofi, che vivono in una grotta un po' emarginati, perché dediti a dispute accese quanto inconcludenti. Una sorveglianza particolare viene loro riservata a causa "dei continui disordini provocati", fra i filosofi, "dalla tribù dei sociologi, i più asociali degli uomini" "143.

Due scienze mantengono nel mondo di domani un peso particolarmente rilevante, la chimica e la psicologia: la prima perché ha costruito un'immagine vivente dell'inanimato, la seconda perché svela la chimica dei sentimenti e delle

\footnotetext{
${ }^{141}$ Ivi, p. 45.

142 Ivi, p. 47.

${ }^{143}$ Ivi, p. 49.
} 
passioni. La psicologia, nei termini semplificatori ipotizzati da Tarde, ha ridotto l'influenza della filosofia e anche della religione: l'unico dogma autorizzato è la credenza nella morte redentrice, con il quale si evita di formare angosce anche intorno alla perdita della vita, che nella credenza diffusa nel futuro costituisce in realtà un ritorno al pensiero. "La morte ci appare come una detronizzazione liberatrice, che restituisce a se stesso l'io decaduto o dimissionario, facendolo ridiscendere nel suo foro interiore, dove guadagna in profondità più di quanto ha perso in dominio esteriore" ${ }^{" 144}$. Il tutto ancora una volta intrecciato a finalità estetiche: la sicurezza diffusa da una tale concezione della morte si unisce, prolungandoli, "al culto della Bellezza e alla fede nell'onnipotenza divina dell'Amore" $" 145$. Tutto sembra dunque comporsi in un quadro armonico, di perfezione indefinita. Ma Tarde conclude il suo apologo con qualche nota di possibile inquietudine: forse anche questa società potrebbe non durare per sempre, per squilibri demografici, per una rilassatezza dei costumi, per un calo del numero delle menti creative, per il sopraggiungere di un sentimento di monotonia. Il finto estensore del Fragment non può tuttavia che registrare come marginali gli eventuali segnali di cedimento: al suo tempo il mondo ideale costruito sotto terra dimostra di poter reggere a lungo.

È importante osservare ancora che la descrizione del futuro di Tarde si allinea alla sua concezione elitaria dei rapporti sociali, che in qualche punto scivola addirittura nel razzismo. Di tipo elitario è anzitutto l'organizzazione politica, sovrastata da un dittatore eletto dai capi delle grandi scuole disciplinari (quelle che abbiamo incontrato prima: pittori, scultori ecc.). La scelta non dà tuttavia mai luogo a particolari problemi o discussioni, perché risulta sempre spontaneo e lineare eleggere il genio superiore. È questo, del resto, il prodotto di un'intera "Repubblica geniocratica", che ha per caratteristica fondamentale "quella di poggiare sull'ammirazione, non sull'invidia; sulla simpatia, non sull'odio; sull'intelligenza, non sull'illusione" ${ }^{\text {"146 }}$. L'ammirazione per il genio governa anche la riproduzione, tradizionale punto delicato di molte utopie. Seguace delle teorie di Malthus, Tarde dichiara che gli uomini del futuro saranno consapevoli dell'inevitabile rapporto fra popolazione e risorse, ancora più rigoroso in un ambiente di potenzialità economiche limitate come quello situato al di sotto della crosta terrestre. Dato questo limite, gli abitanti del futuro non potranno che dedicarsi a un amore casto, in cui vale più l'attesa che la realizzazione, all'interno peraltro di una forte esaltazione dell'amore come sentimento. La donna come soggetto di bellezza è considerata l'unica patria degna di essere amata, in un mondo che non conosce più né conflitti nazionalistici né contese politiche. La necessità del controllo delle nascite e la volontà di mantenere i caratteri superiori

\footnotetext{
${ }^{144}$ Ivi, p. 68.

145 Ivi, p. 69.

${ }^{146}$ Ivi, p. 51.
} 
della specie fanno sì che le donne si possano concedere a fini riproduttivi soltanto a geni riconosciuti, che abbiano prodotto capolavori ritenuti tali anche dai loro rivali nell'arte praticata. Gioco letterario non raro in Utopia, il meccanismo eugenetico si intreccia nel caso di Tarde con qualche colorazione razzistica ${ }^{147}$, collegando il suo autore a quella parte della cultura del suo tempo che utilizza le ricerche antropologiche indotte dal positivismo, per costruirne un'ideologia adatta al momento imperialistico che si sta vivendo nei rapporti fra Occidente e resto del mondo. Per un altro verso, l'attenzione al motivo elitario avvicina Tarde a quello che sarà il suo argomento privilegiato di studio nella vecchiaia: le dinamiche di formazione e di comportamento delle folle.

Nel 1901 Gabriel Tarde pubblica la sua ultima opera importante, L'opinion et la foule, impiegando nell'analisi del tema della folla alcune delle categorie presenti nelle precedenti opere sociologiche e sottolineate attraverso le forme letterarie assunte nel Fragment. Continuatore non inerte delle tesi di Le Bon ${ }^{148}$, Tarde osserva con attenzione i meccanismi psicologici che fanno sì che in una folla gli individui si uniformino a un comportamento comune, per effetto di una regressione all'istintività, e trova la spiegazione principale nella sua consolidata categoria di imitazione, determinata nel caso della folla da spinte emotive. Le altre categorie del suo pensiero sociologico servono poi a classificare i vari tipi di folla, distinguendoli tra folle in prevalenza desideranti, folle credenti e così via: un

${ }^{147}$ Un episodio del romanzo è soprattutto spia del razzismo di Tarde. La società sotterranea riprende l'abitudine degli uomini di esplorare il mondo, il che ora avviene scavando gallerie nelle rocce, sempre più lontane. Durante una di queste "spedizioni" un gruppo di viaggiatori, aprendo un buco in una roccia, si accorge dell'esistenza in un antro di una piccola comunità di cinesi, che hanno evidentemente avuto la loro stessa idea di trovare nel calore sotterraneo una compensazione all'affievolimento della luce solare. I cinesi sono descritti come esseri mostruosi, viventi a uno stato animalesco, dediti al cannibalismo, tanto che i viaggiatori provenienti dalla "civiltà" hanno la tentazione di portare loro guerra. Ma poi prevale l'opinione di rinchiudere il buco che ne ha permesso la conoscenza, la decisione insomma di ignorarli perché indegni di aspirare al titolo di uomini.

${ }^{148}$ La Psychologie des foules di Gustave Le Bon (edizione italiana Milano, Longanesi, 1992) è del 1895. Ricordo che della teorizzazione di Le Bon due sono gli aspetti più rilevanti. Il primo è il meccanismo di identificazione collettiva nella folla, in cui si produce un'unità mentale irrazionale e semplificata, basata sulla sovrapposizione di idee-immagini elementari in cui le folle proiettano senza controlli raziocinanti i propri desideri. Il secondo è l'importanza assunta nelle dinamiche di massa dalla figura del capo ipnotizzatore, portatore di una specie di magia che si estende a mano a mano che si allarga il suo successo: salvo poi il rovesciamento dei comportamenti quando il successo viene meno, allorché la sudditanza al capo si trasforma in disprezzo. Il modello per la teorizzazione di Le Bon è il rapporto fra capo e seguaci che si viene a formare nella Francia della Terza Repubblica attorno a Boulanger. La sociologia di Le Bon concepisce inoltre una forte incidenza di ipotesi razziste: la differenza fra le razze non è tuttavia data da fattori di ordine biologico, alla Gobineau, ma dal carattere morale dei popoli affinato ed ereditato nel corso del tempo, il che induce Le Bon a parlare più di razze storiche che di razze in senso naturalistico. Su questa base anche Le Bon, come Tarde, conferisce un'importanza decisiva alle élites: le razze superiori sono quelle che dispongono di più estese intelligenze al loro interno. Non è tuttavia questa la sede per poter approfondire il discorso intorno al tema della folla, che impegna nel passaggio fra Otto e Novecento anche altre culture politiche oltre quella francese, e in modo significativo proprio quella italiana. Sull'argomento rinvio perciò a Folla e politica, a cura di M. Donzelli, Napoli, Liguori, 1995. 
elemento innovativo rispetto alle prime indagini sui comportamenti spontanei di massa è fra l'altro quello di affiancare al concetto di folla quello di pubblico, inteso come massa dispersa soggetta tuttavia a un'unitaria forma di comunicazione, come avviene per i lettori dello stesso giornale, sottoposti ai medesimi richiami emotivi ${ }^{149}$. Tarde dimostra peraltro una paura più "dialettica" del suo predecessore nei confronti della folla, che Le Bon descriveva come una bestia incontrollabile. Per il nostro sociologo la folla "naturale", cioè formatasi spontaneamente, non annulla del tutto l'autocontrollo individuale, e in ogni caso i suoi scoppi di energia distruttiva sono sempre temporanei e destinati a rientrare abbastanza rapidamente. Diverso è il discorso per le folle "artificiali", organizzate attorno all'imitazione di un modello superiore, spesso di un capo: se i capi intendono volgere il loro potere di ipnotizzazione delle masse ${ }^{150}$ a fini distruttivi della compagine sociale esistente, potranno essere pericolosi proprio in virtù del carattere in qualche modo disciplinato, e perciò continuativo, che il fascino dell'imitazione avrebbe in questa seconda tipologia di folla. Con tale osservazione torna in superficie quell'ambiguità che accompagna tutto il pensiero di Tarde, teorico convinto delle élites, denunciatore delle illusioni ugualitarie che sorreggono il pensiero democratico, erede delle convinzioni disugualitarie di molto positivismo, ma anche capace di preoccuparsi di fronte all'eventualità che delle conoscenze e dei meccanismi di controllo psicologico si approprino volontà malvagie.

\subsection{Il ruolo ambiguo della scienza nell'ucronia di Halévy}

Con l'emergere del problema della folla nella riflessione sociologica giungiamo a una svolta della nostra indagine. L'intuizione comtiana dell'importanza rivestita dai sentimenti nella formazione della coesione sociale sembra essere confermata con l'ingresso delle nuove scienze umane (la sociologia, ma ormai anche la psicologia) nel campo dell'osservazione empirica, pur non ancora sorretta da adeguate metodologie di ricerca. Ma quella che in Comte era soprattutto una fiducia nella possibilità di diffondere sentimenti buoni, alla prova dei fatti dimostra esiti imprevedibili. La determinazione scientifica del peso delle passioni e dei sentimenti nel comportamento delle folle non è solo uno stimolo per operare positivamente nel senso dell'educazione di massa, ma anche la fonte di una consapevolezza che si volge, anziché alla speranza, alla paura di fronte a masse realmente o potenzialmente in preda a pulsioni irrazionali. L'espressione di questo timore (che nelle punte estreme diventa terrore) è propria, a cavallo fra Otto e

\footnotetext{
${ }^{149}$ Segnalo in proposito che nel Fragment la difficoltà di scrivere incidendo la roccia, rispetto a quella di stampare su carta, ha eliminato i giornali, a quanto pare senza grandi dispiaceri da parte degli abitanti.

${ }^{150}$ Vale la pena di ricordare che le prime indagini sociologiche sui meccanismi di condizionamento delle folle nascono in connessione con gli studi sull'ipnosi di Thédule Ribot, autore nel 1896 della Psychologie des sentiments.
} 
Novecento, soprattutto della cultura politica di destra. In attesa che anche tra le forze antiprogressiste si delinei un programma di conquista delle folle, che avverrà specialmente intorno alla crisi della prima guerra mondiale, l'emergere di sentimenti e costumi di massa offre per ora a conservatori e reazionari l'occasione di polemizzare con l'idea politica più collegabile all'avvento delle masse, vale a dire la democrazia. Anche l'ucronia fa la sua parte nel dipingere con preoccupazione la formazione di una società dai comportamenti irrazionali e standardizzati, alla quale contrapporre il desiderio della costruzione (o del ritorno) di un mondo gerarchizzato, in cui i "migliori" sappiano riprendere il controllo dei "peggiori", o sappiano almeno non mescolarsi con le abitudini inferiori.

Un'operazione del genere è tentata in particolare da Daniel Halévy. Appartenente a una famiglia di consolidate tradizioni letterarie, Halévy sarebbe diventato nel corso del Novecento un esponente della cultura liberale di tendenze conservatrici, con più di un punto di contatto con le passioni politiche della destra europea $^{151}$. Quando pubblica, all'inizio del secolo, la sua opera ucronica, Histoire de quatre ans (1997-2001), Halévy manifesta tuttavia una militanza culturale meno decisamente allineata in senso conservatore: vicino per un verso a Péguy e per l'altro a Sorel, si è schierato dalla parte degli intellettuali dreyfusardi e si accinge a partecipare alla stagione del sindacalismo rivoluzionario. Egli professa un ambiguo programma di socialismo nazionale in cui si fa sentire qualche sintomo preparatorio del conservatorismo della maturità (come l'enfasi sulle virtù del lavoro nazionale, o la polemica contro le degenerazioni della democrazia parlamentare), sorvegliato tuttavia da una sincera attenzione per la questione sociale. E questa attenzione appare più un frutto della tradizione del socialismo cooperativistico che non un riflesso della protezione degli inferiori predicata dagli ambienti di destra.

La prima edizione dell' Histoire vede la luce alla fine del 1903 nei "Cahiers de la Quinzaine" diretti da Charles Péguy. Per il trentenne Halévy la pubblicazione nella prestigiosa rivista di Péguy non è un caso: è l'adesione a un corpo di idee di cui sente interamente il fascino. Per quanto successivamente indicato dalla destra nazionalista francese come uno dei suoi padri ispiratori, Péguy (come un tempo

\footnotetext{
${ }^{151}$ Daniel Halévy nasce nel 1872; il padre, Ludovic, è un noto autore di testi teatrali: fra l'altro, è il librettista della Carmen di Bizet. Anche il fratello di Daniel, Elie, è uno scrittore, in particolare di studi e memorie di argomento politico. Prima dell'Histoire qui esaminata, Daniel Halévy pubblica nel 1900 un interessante Essai sur le mouvement ouvrier en France, segno dei suoi originari interessi in campo sociale. Intorno al 1910 comincerà tuttavia una propria marcia di avvicinamento prima alla cultura volontaristica, con una biografia di Nietzsche, poi a un socialismo di impianto sempre più tradizionalista, con studi su Péguy e Proudhon. Il suo conservatorismo traspare anche nelle opere e negli atteggiamenti della vecchiaia (Halévy morirà nel 1962). Nel 1948 pubblica un Essai sur l'accélération de l'histoire, volto a propagandare l'idea della necessità di un'azione umana capace di controllare il progresso, da considerarsi pericoloso quando è antitetico rispetto ai valori tradizionali. Negli stessi anni Halévy spende la propria residua influenza per rivendicare la revisione del processo con il quale Maurras era stato condannato all'ergastolo per collaborazionismo alla fine della seconda guerra mondiale.
} 
Comte) non è facilmente assimilabile agli schieramenti politici in auge. La sua militanza culturale aggiunge ai riferimenti tipici delle forze tradizionaliste (la Francia cristiana del medioevo e gli antichi costumi popolari) la necessità di un incontro con parte dei valori emersi durante la Rivoluzione del 1789. La sua mitizzazione di Giovanna d'Arco non ha nulla a che vedere con la stanca ripetizione di un'icona reazionaria che sarà propria della destra francese nel Novecento: per Péguy è il simbolo dell'unione fra popolo, cristianità e nazione in lotta per i propri diritti. Il richiamo tradizionalista alla provincia sana e l'appello all'ordine sono in qualche modo bilanciati da un' accentuazione del lavoro come unica fonte del merito sociale, e da una concezione del popolo non disgiunta da talune suggestioni di democrazia socialista.

Analoghe ambiguità si trovano nelle prime opere di Halévy. La sua polemica contro la democrazia parlamentare e contro il socialismo di matrice marxiana, lo apparentano certo con la cultura di destra del suo tempo. Ma la ricerca di forme di emancipazione dei lavoratori diverse dalla rivoluzione proletaria non ha ancora assunto in lui l'aspetto della rassegnazione conservatrice. E anche intorno all'argomento che qui ci interessa maggiormente, quello dei legami fra scienza e morale, la posizione di Halévy presenta come vedremo segni di profonda ambivalenza. Interpretare Histoire de quatre ans soprattutto come un'invettiva antidemocratica, come è tradizionale nella critica italiana intorno a questo testo ${ }^{152}$,

${ }^{152}$ La prima edizione italiana dell'Histoire risale al 1911, quando esce nei "Quaderni della Voce" per iniziativa di Giuseppe Prezzolini, con il titolo allusivo di Il castigo della democrazia. Il titolo vuole suggerire un'interpretazione del testo ancorata appunto a valorizzare le convinzioni antidemocratiche in esso presenti, anche se la premessa all'edizione italiana non è priva di qualche passaggio ambiguo a questo proposito. Vi si legge per esempio che il libro di Halévy non è "una fantastica anticipazione storica. Le radici di questo racconto si addentrano nel terreno della realtà sociale presente. È un quadro della degenerazione democratica e insieme un processo di morale rigenerazione, un bilancio delle energie di ogni democrazia. Perciò lo abbiamo intitolato Il castigo della democrazia, quantunque l'autore ci abbia fatto notare che può esistere una democrazia puritana". Il titolo Il castigo della democrazia (Storia di quattro anni 1997-2001) è ripreso nella più recente edizione italiana: Roma, Volpe, 1971. Il curatore di quest'ultima edizione, Gianfranco de Turris, fa seguire al testo di Halévy un proprio saggio su Utopia e antiutopia, in cui avanza uno schema di classificazione degli scritti intorno all'utopia tale da permettere di dare un valore "positivo", e non solo di opposizione, a opere come quella di Halévy. De Turris distingue fra utopia e antiutopia facendo riferimento non alle tecniche dell'immaginazione, ma alle intenzioni ideologiche degli autori. Secondo lui il significato essenziale dell'utopia classica (di Moro, per intenderci), che nelle sue varianti moderne diventa patrimonio della cultura socialista, risiede nella costruzione di modelli di organizzazione comunitaria di tipo ugualitario, e quasi sempre basati sulla socializzazione della proprietà dei beni e della vita economica in genere. Ė questa l'utopia positiva, assimilabile nelle intenzioni all'utopia "negativa", che per de Turris è il genere che vuole denunciare la mancanza della democrazia, descrivendo una tirannide utopica che rende gli uomini uguali nella forma negativa di una comune sottomissione. Esempi di tale genere potrebbero essere Il tallone di ferro di Jack London o Fahrenheit 451 di Ray Bradbury, con la loro denuncia dei mali provocati da regimi autoritari, in mano a pochi potenti. L'antiutopia è invece quella letteratura che combatte le ragioni e le aspirazioni degli utopisti, perché le ritiene basate su ipotesi impossibili o pericolose. Per de Turris l'antiutopia è negativa quando rappresenta le degenerazioni provocate da un'utopia comunitaria data come realizzata, come è nel caso delle famose opere novecentesche di Huxley e di Orwell. Ma esisterebbe anche un'antiutopia 
è senz'altro lecito, perché costituisce lo scopo dichiarato dello scritto. Ma è opportuno tenere in considerazione, accanto all'ispirazione elitaria dal sapore antidemocratico e con preoccupanti livori razzisti, anche gli altri umori presenti nelle pieghe del racconto, a partire proprio dall'odio-amore manifestato da Halévy nei confronti della scienza.

La prima parte dell'Histoire spiega come, già nella prima metà del Novecento, si sono creati i presupposti che condurranno a fine secolo in Francia a un'apocalittica crisi morale. La chiave di volta della degenerazione sociale è la capacità della scienza e dell'industria di produrre albumina a basso costo a fini alimentari. Questa innovazione ha in apparenza un segno positivo, liberando la popolazione dall'onere di dover lavorare a lungo per raggiungere la sopravvivenza: nella Francia di fine Novecento bastano in media tre ore di lavoro al giorno per mantenere l'equilibrio produttivo. Ma la realtà sociale è diversa dall'ottimismo che un'innovazione del genere poteva indurre. La riduzione delle necessità di lavoro, a cominciare da quello agricolo, svuota le campagne della loro presenza moralmente più significativa: il popolo operoso. Ma anche nelle città, dove la popolazione si concentra, le classi lavoratrici non manifestano più l'influenza etica del passato. "A dire il vero, il popolo, questo grande fanciullo che costituiva una riserva di vitalità per le civiltà antiche, non esisteva più. Tutti i tipi rappresentativi della vecchia umanità si erano fusi in un tipo unico, molto simile all'impiegato del XIX secolo, gaudente e frollo, in abiti borghesi" ${ }^{\prime 153}$. La fine della sfida per la sopravvivenza apre drammaticamente agli uomini il problema di come trascorrere il tempo. "Si vide allora che la soppressione della miseria, anziché risolvere i problemi dell'umanità, li rimetteva invece tutti sul tappeto. [...] Quelle moltitudini, una volta bisognose, che cosa avrebbero fatto delle loro anime e dei loro corpi inerti? L'impiego delle ore di riposo, diventò la più urgente di tutte le questioni sociali"154.

Su questa osservazione si innesta la parte apocalittica dell'opera di Halévy. Liberata dallo stimolo del bisogno, l'umanità si dedica a quelli che per l'autore dell'Histoire sono i peggiori vizi: gli eccessi sessuali, il consumo di droghe, l'abuso dell'alcool, la frequentazione di spettacoli cruenti (a Parigi esiste perfino un Colosseo dove combattono i gladiatori ...). La degradazione morale degli abitanti, che cominciano a evidenziare la crescita di malattie e squilibri psichici dovuti in particolare all'uso delle droghe, è dipendente da due fattori che si alimentano a vicenda: il dominio dei monopoli in economia e l'estendersi della

\footnotetext{
"positiva", che combatte i valori ugualitari rappresentando come desiderabile una società di tipo gerarchico: cosa che almeno in parte farebbe appunto Halévy, come vedremo più avanti. Resta inteso che questa nozione di antiutopia "positiva" esprime, oltre al tentativo di una classificazione critica, anche un giudizio di valore, sottinteso nel ritenere una società gerarchica come augurabile e propositiva, non espressione di pura reazione negativa. Non per nulla de Turris, per sostenere la sua tesi, ricorre alle riflessioni operate prima di lui intorno all'utopia da scrittori di destra come Tilgher e Cioran.

${ }^{153}$ D. Halévy, Il castigo della democrazia, cit., p. 29.

${ }^{154}$ Ivi, p. 11.
} 
democrazia parlamentare in politica. Si è infatti creato un corto circuito per il quale il governo politico asseconda le peggiori inclinazioni delle masse, le quali a loro volta scelgono come loro rappresentanti gli uomini politici che meno promettono di ostacolare la rincorsa all'esplosione degli istinti. Il tutto è sorvegliato nell'ombra dal trust dei produttori di albumina, e in secondo luogo dagli interessi economici che ruotano attorno agli oggetti del desiderio popolare. Una campagna di moralizzazione tentata nel 1945 dai pochi illuminati rimasti, racconta Halévy, è per esempio fallita per la contropropaganda messa in atto dai monopolisti dell'alcool e della morfina, che sono anche proprietari dei caffè e organizzatori degli spettacoli. E il già dreyfusardo Halévy afferma ora che tutti questi interessi fanno capo al capitalismo ebraico, rivelando così un'intonazione antisemita che lo apparenta non solo con i luoghi comuni della destra del tempo, ma anche con quella parte oscura della polemica anticapitalistica che si era espressa con toni antisemiti anche in esponenti del pensiero socialista, primo fra tutti Proudhon.

L'immagine di fondo restituita da Halévy di un ipotetico progresso economico è dunque quella della decadenza morale generalizzata, provocata dagli interessi capitalistici e da un oscuramento massiccio delle coscienze. La scienza ha una responsabilità in tutto ciò, in quanto asservita alle ragioni del profitto economico. Ma, nei termini ambivalenti cui si faceva cenno all'inizio, Halévy affida una qualche speranza di riscatto ancora alla scienza, se depurata dei suoi elementi di sudditanza all'interesse economico. A resistere alla corruzione generale sono infatti soltanto piccoli gruppi di esseri moralmente superiori: gli esponenti di un cattolicesimo minoritario, con aspirazioni ascetiche (mentre il Vaticano è passato in mano alla stregoneria); i virtuosi che hanno dato luogo a colonie di tipo cooperativo; e appunto gli scienziati che hanno scelto di non porre le proprie conoscenze a disposizione esclusiva dei potentati economici.

Halévy parla infatti, a proposito della Francia del futuro in preda alla degenerazione, della resistenza posta in essere da due comunità di scienziati, vere $\mathrm{e}$ proprie élites razionali e morali. La prima fa capo a Rabaud, studioso delle leggi dell'ereditarietà e convinto negatore dell'idea di libertà, attorno al quale si schierano coloro che l'autore dell'Histoire definisce positivisti autoritari: il loro umanesimo controrivoluzionario è sintetizzato dal motto "Disciplina, Gerarchia, Amore". Un altro gruppo di scienziati ruota attorno al chimico Tillier, il vero protagonista del racconto di Halévy: significativamente rappresentato come insediato nel laboratorio di chimica a suo tempo diretto da Berthelot, Tillier esprime le stesse preoccupazioni dei positivisti autoritari a proposito del degrado in atto, ma non ha abbandonato del tutto il punto di vista democratico. Egli è peraltro in sintonia non "con la democrazia corrotta del suffragio universale, ma con la democrazia organizzata delle associazioni operaie" ${ }^{155}$. Per quanto Tillier si professi un positivista, a lui guardano con fiducia i giovani delle colonie associative, il cui

\footnotetext{
${ }^{155}$ Ivi, p. 27.
} 
movimento è indicato con la denominazione di "socialisti libertari". Fra i loro obiettivi, ai quali non sono insensibili anche i positivisti autoritari, vi è quello di proporre un uso del tempo libero votato all'elevazione culturale, anziché all'abbrutimento collettivo ${ }^{156}$. Nelle varie corporazioni scientifiche, come si è fatto cenno, predomina un acuto sentimento elitario, che si prolunga in un aperto disprezzo per le masse che, nonostante tutti gli avvisi della scienza, si lasciano precipitare verso un godimento incontrollato. La coscienza della loro diversità si accompagna tuttavia, nei vari spiriti "eletti", alla consapevolezza della complessità delle cose ("gli uomini sono delle povere creature disorientate, nel nuovo mondo della scienza" $" 157$ ) e alla speranza di poter guidare il difficile ma inevitabile adattamento dell'uomo al progresso tecnico, visto che è impensabile il semplice ritorno alla natura.

Per il momento le masse si dimostrano tuttavia sorde agli appelli alla moderazione degli scienziati. Anche in Halévy, come negli altri romanzi che abbiamo ricordato al paragrafo precedente, il nodo determinante della narrazione si stringe attorno a una catastrofe. Nel 1997 esplode un'epidemia, che nel giro di tre anni falcidia la popolazione in tutto il mondo occidentale. I gruppi igienisti riescono tuttavia a rimanere almeno in parte al riparo dalla catastrofe, in virtù del loro relativo isolamento rispetto ai costumi dominanti, e al termine dell'epidemia si scoprono nuova classe dirigente, in una società contrassegnata da un brusco calo demografico. Tillier, dopo aver superato una lunga malattia e aver unito alla propria fede scientifica lo studio della Bibbia, viene chiamato a occupare l'incarico prestigioso di ministro dell'istruzione pubblica, altra carica che era stata effettivamente, come sappiamo, ricoperta da Berthelot. E fra gli elementi di ambiguità di Halévy, è appunto il caso di rilevare la persistenza del richiamo a un personaggio come Berthelot, di sentimenti democratici e ottimisticamente convinto delle possibilità del progresso, all'interno di un'interpretazione sociale di tipo invece autoritario e angosciata rispetto alle prospettive future.

La società immaginaria che Halévy descrive come succeduta alla degenerazione e alla crisi del vecchio mondo, è basata sui caratteri elitari, con venature razzistiche, propri delle sue convinzioni più profonde. Intorno all'anno 2000 al governo della Francia si trovano i positivisti autoritari, che hanno conquistato il potere con l'aiuto dei socialisti libertari. La struttura sociale da essi costruita è di tipo fortemente aristocratico. Non esistono più classi aperte in senso capitalistico, ma caste chiuse: al vertice sono i societari, in posizione intermedia i collegiati, in

\footnotetext{
${ }^{156}$ Come è noto, nell'ambito dell'economia politica il primo a mettersi nell'ottica di uno stato "stazionario", per il quale l'esaurimento temporaneo delle spinte produttivistiche non era da considerarsi in sé una condizione solo negativa, era stato John Stuart Mill. Nei Princìpi di economia politica si legge che la riduzione della giornata lavorativa, indotta da un progresso tecnico in grado di rendere meno esteso il bisogno di lavoro per la sopravvivenza, avrebbe potuto creare una disponibilità di tempo per l'istruzione della classe operaia, e quindi per un'elevazione delle condizioni culturali della società nel suo complesso.

${ }^{157}$ D. Halévy, Il castigo della democrazia, cit., p. 41.
} 
fondo alla scala sociale i coatti. Quest'ultimi, infetti, sono aiutati a morire presto, ma dolcemente. Le leggi matrimoniali impongono di fatto unioni solo all'interno delle singole caste: chi sceglie di sposare individui di casta inferiore scende anch'egli nella fascia più bassa. L'organizzazione sociale adottata corrisponde alle intenzioni dei gruppi che in precedenza esprimevano il loro dissenso contro i costumi licenziosi, e che ora non intendono mescolarsi in alcun modo con le masse.

Le élites che governano la società dei sopravvissuti all'epidemia non sono peraltro del tutto d'accordo sull'ideologia imperante. Mentre i positivisti autoritari hanno avuto modo di organizzare la società gerarchica e illiberale da loro teorizzata come l'unica adatta a preservare l'umanità, i socialisti libertari avanzano dubbi sugli aspetti più apertamente antidemocratici del nuovo sistema, riproponendo echi del vecchio socialismo umanitario. Alle obiezioni che una loro esponente, Claire, avanza contro le tesi gerarchiche ed eugenetiche, un giovane che ne è invece convinto risponde nei termini seguenti: "L'antica società livellava: questa era la sua legge. La nostra conosce le vere ineguaglianze: è la sua legge ed è la vera. E perché dunque l'ineguaglianza, Claire? Tu concepisci un livello al quale fissare l'umanità? Puoi essere più felice di quando lavori con un buon capo, quando gli obbedisci? Per me, l'ideale non è l'uguaglianza; è la superiorità: superiori che mi diano ordini, e inferiori ai quali io dia i miei ordini. E la libertà? Claire, perché la libertà? Che cos'è dunque questa grande parola? La libertà di uno sciocco genera sciocchezze, quella d'un forte produce forza; e l'ideale è là: più forza e maggiore coscienza" ${ }^{\prime 158}$.

L'allievo di Péguy rinuncia in questo caso a cercare di saldare i valori della tradizione con quelli della rivoluzione: la requisitoria finale contro uguaglianza e libertà è anzi eloquente, e risente sia delle posizioni in tal senso già espresse dal positivismo comtiano, sia delle nuove suggestioni del volontarismo superomistico alla Nietzsche e alla Sorel. L'utopia elitaria di Halévy perde tuttavia uno dei caratteri tipici del genere, quello della stabilità. Le idee dei positivisti autoritari non diventano, nella Francia del futuro, convinzione generale: i socialisti libertari mantengono una distinzione dottrinaria, anche se, dopo aver aiutato gli alleatiavversari nella ricostruzione dopo l'epidemia, faranno altrettanto al profilarsi di una nuova minaccia, l'avanzata da Oriente di una nuova possibile barbarie, quella dei russi intenzionati a espandersi. La conclusione dell'Histoire induce alla speranza sulla vittoria dell'Occidente, come traspare dalle parole di Tillier: "Io credo che noi vinceremo. Noi, voglio dire, l'élite umana, le razze che sanno ragionare e organizzare, le razze morali. Esse vinceranno perché il loro impegno è uno solo. I loro sforzi, per quanto sparsi, si integrano fra loro" ${ }^{\text {"159 }}$. Ma anche questa speranza non è incondizionata, non ha la fissità delle previsioni utopiche "forti". Il romanzo si conclude con l'uccisione di Tillier in un attentato eseguito da un russo,

\footnotetext{
${ }^{158}$ Ivi, pp. 98-99.

${ }^{159}$ Ivi, p. 119.
} 
suo ex allievo: un epilogo che simboleggia un progresso morale soggetto a una lotta mai definitivamente chiusa.

Con Halévy le ambiguità su cui si era formato mezzo secolo prima il messaggio morale del positivismo sembrano sciogliersi verso una polarità: quella della radicalizzazione dei motivi autoritari, della polemica contro libertà e uguaglianza, della distinzione fra minoranze consapevoli e superiori e masse subalterne non solo al potere politico e sociale, ma ora anche al nuovo potere della scienza. Gli anni di Tarde e di Halévy sono del resto anche gli anni in cui si appropriano di Comte i teorici di un nazionalismo aggressivo, virulento e accesamente antisemita, il nazionalismo di personaggi come Drumont, Léon Daudet, Barrès, Maurras, qui tralasciati perché estranei alla dimensione utopica, ma attivi nel costruire un profilo compiuto del lascito comtiano come eredità religiosa di ispirazione gerarchica. Certo, l'uso dell'impianto positivista da parte di simili scrittori si rivela spesso un abuso, con la sua equivalenza fra religione dell'Umanità e ritorno al cattolicesimo, o fra passaggio a élites scientifiche e restaurazione monarchica. E, quando esiste, il richiamo alle nuove scienze dell'uomo si rivela spesso, nell'interpretazione autoritaria, bassamente strumentale, come avviene con la ricerca nelle antropologie razziste della conferma di aprioristiche convinzioni disugualitarie.

Con questi punti deboli, l'ucronia riesce comunque a comunicare con una certa efficacia le difficoltà di governo sociale che un mondo avviato a una crescente tecnicizzazione è destinato a proporre, nella sua esaltazione di un successo materiale diffuso. Talune suggestioni positiviste vengono, da molte voci della letteratura ucronica, impiegate paradossalmente per togliere valore al progresso, anziché per riproporre in termini aggiornati il complesso della sfida comtiana, quello di come coniugare il progresso con l'ordine, l'evoluzione tecnico-scientifica con il controllo politico e morale. La risoluzione nell'immaginario delle contraddizioni positiviste non assume tuttavia, nel passaggio fra Otto e Novecento, la sola veste della reazione. Come vedremo all'inizio del prossimo capitolo, c'è anche chi riprende il motivo scientista per tentare di saldarlo con un ceppo utopico di grande tradizione: quello socialista. 


\section{I conti con l'utopia}

\subsection{Un modello di socialismo tecnologico: Zola e il riscatto dei lavoratori}

Negli stessi anni in cui la letteratura ucronica si piega verso l'illusione di un mondo che arresti il progresso, temuto come un pericolo per le qualità umane, l'intreccio fra scienza e morale ritrova un terreno di rappresentazione positiva in una letteratura che si riavvicina ai canoni più tipici dell'utopia. Accanto a una cultura neoconservatrice si affaccia infatti un programma di ripresa delle aspirazioni e dei desideri di matrice socialista, in particolare del socialismo utopistico della prima metà dell'Ottocento, da ridefinire ora alla luce delle speranze sul progresso scientifico distribuite dalla cultura positivista.

Ancor prima della fine del secolo XIX, a impegnarsi maggiormente su questo terreno è la variante del positivismo che si sviluppa negli Stati Uniti d'America. Si tratta di una variante che trova nella filosofia del posivitismo in generale, e nella predicazione di Comte in particolare, suggestioni in grado di proporre antidoti agli eccessi di individualismo, indotti da un progresso economico realizzato sotto il segno di un capitalismo "anarchico" come quello americano ${ }^{160}$. In genere il positivismo americano ricerca nelle idee comtiane di sociocrazia e di religione dell'Umanità strumenti di coesione sociale, in grado di bilanciare con richiami ai

\footnotetext{
${ }^{160}$ L'interpretazione comtiana della storia, con la sua insistenza sulla disciplina sociale e sull'incontro fra le classi, costituisce negli Stati Uniti un fattore di riequilibrio rispetto all'idea dell'evoluzione sociale spontanea propagandata dagli ammiratori di Spencer. Un posto particolare in questa operazione spetta a Lester Frank Ward, autore nel 1883 di Dynamic Sociology, al quale si può far risalire l'ingresso nella cultura americana non solo delle categorie sociologiche comtiane, ma anche dei primi rudimenti di una teoria del Welfare. Scrive Brunella Casalini che in America "il comtismo trovò un terreno fertile nel desiderio di rafforzare la coesione sociale e di temperare le spinte verso un individualismo atomistico e tendenzialmente anarchico". Negli ultimi decenni dell'Ottocento un'ipotesi del genere risultava rilevante sia come tentativo di sanare le ferite della guerra di secessione, sia come risposta al desiderio di "far fronte alla crescita di un'economia sempre più caratterizzata da forti concentrazioni industriali e da un quadro sociale il cui tratto distintivo era dato dall'emergere di quel conflitto di classe da cui l'America si era considerata immune" (L'interpretazione della "sociocrazia" nel pensiero di Lester Frank Ward, in Sociologia, politica e religione, cit., pp. 151-152). Sul tema generale della ricezione del pensiero di Comte in America si veda soprattutto G.J. Harp, Positivist Republic. Auguste Comte and the Reconstruction of American Liberalism. 1865-1920, The Pennsylvania University Press, 1995.
} 
doveri di solidarietà un sistema basato sull'iniziativa individuale, e quindi soggetto in permanenza a spinte disgregatrici. In alcuni casi questa ricerca di una disciplina comunitaria sfocia in veri e propri modelli utopici, socialisteggianti e tecnocratici insieme, come è il caso dei Diothas di Ismar Thiusen ${ }^{161}$ e soprattutto del romanzo utopico più famoso prodotto dal positivismo americano, e cioè Looking Backward di Edward Bellamy. Quando nel 1888 pubblica la sua opera utopica, Bellamy ha già al suo attivo una ricognizione sulla religione comtiana (ribattezzata, dal titolo di un suo libro del 1873, Religion of Solidariety) in cui una visione trascendentalista della natura, come luogo infinito dal quale proviene la vita e al quale ritornano le anime dopo la morte dei corpi, si accompagna all'appello agli uomini a elevarsi a una dimensione divina per mezzo della solidarietà fra tutti gli esseri viventi. Con Looking Backward il richiamo di ordine religioso si concretizza nella descrizione di una società ideale, nella quale alcuni elementi del tradizionale pensiero socialista (come l'uguaglianza e la proprietà comune dei mezzi di produzione) cercano di convivere con il governo tecnocratico imposto dal bisogno di competenza proprio delle società moderne, scientificamente progredite ${ }^{162}$.

Un cammino analogo è percorso in Francia dallo scrittore più influente di fine Ottocento, Émile Zola, che rinviene, nell'idealizzazione di un incontro fruttuoso fra

${ }^{161}$ Thiusen, pseudonimo di John Macnie, è un matematico inglese trapiantato negli Stati Uniti, dove nel 1883 pubblica The Diothas, storia di una popolazione che vive in una società ideale posta 7500 anni dopo il tempo dell'autore. La comunità dei Diothas vive in un'armonia costruita insieme dalla legge e dalla scienza.

162 Di Looking Backward 2000-1887 possediamo un'edizione italiana: E. Bellamy, Guardando indietro 2000-1887, a cura di E. Malagoli, Torino, Utet, 1957. Protagonista del romanzo è Julian West che, addormentatosi nel 1887 per un sonno ipnotico, si risveglia nel 2000 e assiste così a una società riorganizzata su basi comunistiche, per effetto non di una rivoluzione violenta bensì della progressiva illuminazione dell'opinione pubblica. L'America del 2000 si è lasciata alle spalle il modello competitivo dell'Ottocento, per costruire un sistema insieme socialista (proprietà pubblica dei mezzi di produzione) e tecnocratico (ruolo dirigente affidato agli scienziati, largo impiego di innovazioni tecniche, specialmente nel campo dei trasporti e delle comunicazioni). Il progresso tecnico si coordina comunque con una concezione della vita di tipo salutistico e naturalistico, come è costume dell'utopismo americano. Una caratteristica dell'utopia di Bellamy è la formazione di una specie di esercito industriale, che impiega in ambito produttivo simboli e gradi di derivazione militare. Ogni cittadino è tenuto a osservare il dovere del lavoro dai 21 ai 45 anni: gli anni precedenti questo intervallo servono all'istruzione, quelli successivi danno diritto al pensionamento. Al vertice del sistema stanno un presidente e un ispettorato (sorta di governo dei tecnici) eletti dagli anziani (coloro che, avendo superato il quarantacinquesimo anno di età, hanno abbandonato il lavoro). Nel 1897 Bellamy scrive un seguito a Guardando indietro, intitolandolo Equality: la notorietà di queste sue opere utopiche è confermata dal fatto che appena un anno dopo esce la traduzione italiana, Uguaglianza (Palermo, Sandron). In Uguaglianza il nostro autore offre maggiore attenzione, rispetto all'opera precedente, al tema dell'educazione e delle convinzioni religiose. La comtiana religione dell'umanità viene descritta come rafforzata da un forte sistema di istruzione, in cui si insegnano i valori dell'obbedienza, della disciplina e della dedizione al dovere, mentre la donna è oggetto di un'autentica venerazione, giusto riconoscimento del suo ruolo di educatrice (in società, peraltro, e non soprattutto in famiglia come era per Comte). Sullo scrittore americano rinvio agli studi di A.E. Morgan, Edward Bellamy, New York, Columbia University Press, 1944, e di S.E. Bowman, Edward Bellamy, Boston, Twayne, 1986. 
la tradizione ugualitaria del socialismo e lo sviluppo tecnico e scientifico consentito dalla cultura positivista, lo scenario utopico nel quale ambientare la soluzione della questione sociale. Non è questa la sede per ricostruire estesamente il profilo della cultura zoliana, che oltretutto compete più agli storici della letteratura che a quelli del pensiero politico ${ }^{163}$. Mi limito perciò a ricordarne e a sottolinearne pochi aspetti, a partire dalla nota contiguità fra il programma letterario di Zola e le considerazioni avanzate dalla scienza del suo tempo, come appaiono in particolare nel saggio teorico da lui dedicato al Roman expérimental. Di questo saggio vanno peraltro valutate appieno anche le ambivalenze, che Zola sembra ereditare direttamente dalla fattispecie comtiana della cultura positivista. Da un lato il grande romanziere esplicita la sua adesione a una concezione della letteratura di tipo "scientifico", incentrata sulla convinzione dell'origine biologica dei fatti sociali e degli atteggiamenti mentali, e del loro conseguente carattere deterministico $^{164}$. Ciò comporta la necessità di risalire per ogni evento alle condizioni ambientali di maturazione, ricostruite da un narratore che deve adeguarsi integralmente a un costume di oggettività impersonale, come dovrebbe accadere per ogni scienziato di fronte all' argomento del suo studio. Da qui deriva, fra l'altro, la celebre definizione del romanzo come "esperimento" guidato dall'osservazione, che ricorda nell'espressione zoliana analoghe indicazioni di metodo presenti nelle scienze della natura come in quelle dell'uomo. Accanto a questo "naturalismo" deterministico (nel senso di riduzione della materia psichica a fenomeno biologico) convive però d'altro lato, nel pensiero di Zola, un'aspirazione a coniugare la ragionevole certezza del progresso con l'opportunità di utilizzare le crescenti conoscenze scientifiche per governare il cammino umano. L'oggettività

${ }^{163}$ Colgo l'occasione per segnalare qualche studio critico su Zola: E. Scolari, Arte e scienza nell'estetica di Zola, Reggio Emilia, Age, 1981; H. Mann, Zola, a cura di G. Chiarini, Napoli, Pironti, 1984; J. Best, Expérimentation et adaptation: essai sur la méthode naturaliste d'Émile Zola, Paris, Corti, 1986; Il terzo Zola: Émile Zola dopo i Rougon-Macquart, a cura di G.C. Menichelli, Napoli, Istituto Universitario Orientale, 1990; H. Troyat, Zola, Paris, Flammarion, 1992; A. Pagès, Émile Zola: bilan critique, Paris, Nathan, 1993; C. Becker, G. Gourdin-Servenière, V. Lavielle, Dictionnaire d'Emile Zola: sa vie, son oeuvre, son époque, Paris, Laffont, 1993.

${ }^{164}$ In un famoso passo del Roman Zola scrive, a proposito della derivazione della sua letteratura dall'ambiente scientifico dell'epoca: "La scienza prova che le condizioni di esistenza di un fenomeno sono le stesse negli organismi viventi e nei corpi bruti; ed allora la fisiologia assume a poco a poco la certezza della chimica e della fisica. Ma ci si fermerà a questo punto? Certamente no. Quando avremo provato che il corpo dell'uomo è una macchina di cui un giorno si potranno smontare e rimontare gli ingranaggi a piacimento dello sperimentatore, si dovrà ben passare alle manifestazioni passionali e intellettuali dell'uomo. Da quel momento entreremo nel dominio che, fino ad ora, apparteneva alla filosofia ed alla letteratura; sarà la conquista decisiva, da parte degli scienziati, delle ipotesi dei filosofi e degli scrittori. Vi sono la fisica e la chimica sperimentali; vi sarà la fisiologia sperimentale $\mathrm{e}$, più tardi ancora, si avrà il romanzo sperimentale. Si tratta di una progressione inevitabile ed è facile prevederne fin da ora il termine finale. Tutto è collegato, bisognava partire dal determinismo dei corpi inanimati per arrivare al determinismo degli organismi viventi; e poiché scienziati come Claude Bernard dimostrano ora che leggi immutabili regolano il corpo umano, si può annunciare, senza timore di ingannarsi, il momento in cui a loro volta saranno formulate le leggi del pensiero e delle passioni” (E. Zola, Il romanzo sperimentale, Parma, Pratiche, 1980, pp. 12-13). 
nell'avvicinamento alla materia sociale non è quindi soltanto una questione di conoscenza, ma anche di dominio: la scienza diventa in questo processo lo strumento principale per "divenire padroni della vita per dirigerla" 165 .

Per quanto il programma di indagine di Zola si volga verso tutte le classi sociali del suo tempo, protagoniste di volta in volta dei suoi romanzi, il progetto di fare della conoscenza scientifica uno strumento di riscatto, oltre che di spiegazione della realtà, trova un campo di applicazione privilegiato a proposito delle classi lavoratrici. In termini analoghi a Comte, il problema più grave per Zola, in rapporto alla classe operaia del suo tempo, è quello della povertà e dell'insicurezza, temi attorno ai quali si può allineare più di un romanzo del nostro scrittore, compresi alcuni fra i più celebrati: come Assommoir, ovvero la denuncia delle condizioni disumane prodotte dalla povertà; Germinal, ossia l'illustrazione del momento della rivolta da parte del proletariato sfruttato: e Travail, cioè la rappresentazione in chiave utopica della giustizia che potrebbe sanare i mali della condizione sociale esistente. Seppur meno famoso degli altri due, è proprio Travail il romanzo che più ci interessa a proposito dell'incontro in sede utopica fra socialismo e positivismo, che Zola fa scaturire letterariamente dal confronto critico con la realtà dell'effettiva società capitalistica del suo tempo ${ }^{166}$.

La vicenda narrata in Travail, per quanto disegni un complesso affresco sociale teso a illustrare un'intera società industriale, ruota per gran parte attorno alla figura di un ingegnere, Luc Froment. Di origine proletaria, e quindi sensibile alla questione operaia, Luc ritorna alla cittadina natale, Beauclair, chiamato da un amico, Martial Jordan, uno scienziato che si applica nella ricerca incessante del miglior impiego dell'energia elettrica come strumento di riduzione della fatica del lavoro. Jordan vive con una sorella di consolidate convinzioni umanitarie, che, insieme con i due tecnici e pochi altri spiriti illuminati, completa il nucleo "positivo" del romanzo dal lato della borghesia: e cioè un piccolo gruppo di scienziati di sentimenti progressisti, con una spiccata e generosa attenzione per i problemi dei lavoratori. Un'altra presenza positiva, che si snoderà nella prosecuzione del romanzo, è quella proletaria: nelle file operaie Luc troverà in seguito sia alleanze decisive per i suoi progetti, sia l'amore di una compagna appassionata, che diverrà sua moglie e madre dei suoi figli.

Jordan ha chiamato Froment per avere da lui consigli e appoggi di fronte all'emarginazione che sta subendo dalla vita produttiva della cittadina. L'economia locale è incentrata sul predominio di una grande acciaieria ${ }^{167}$, che attraverso

\footnotetext{
${ }^{165}$ Ivi, p. 16.

${ }^{166}$ Dopo essere apparso come feuilleton nell' "Aurore", Travail esce per la prima volta in volume nel 1901, come parte dei Quatre Évangiles. Ne è disponibile un'edizione anastatica (Paris, L'Harmattan, 1993, con prefazione di T. Paquot), dalla quale traggo le citazioni che seguono nel testo.

${ }^{167}$ Come si sa, e come è confermato dai suoi Taccuini (Torino, Bollati Boringhieri, 1987), Zola aveva l'abitudine di documentarsi "sul campo" intorno all'ambientazione dei suoi romanzi. La vicenda narrata in Travail prende spunto, almeno per quanto riguarda gli aspetti tecnici della produzione e del lavoro, da una visita compiuta dallo scrittore alle famose acciaierie Schneider di Le Creusot.
} 
complessi giochi di eredità e compravendite è passata in mano a proprietari che impersonano i peggiori caratteri egoistici del capitalismo. Quando Luc giunge a Beauclair si è appena concluso un lungo sciopero con una sconfitta rovinosa degli operai, costretti, anche per la repressione messa in atto dalla gendarmeria, a riprendere il lavoro alle condizioni imposte dall'azienda. La prima parte del romanzo, incentrata sulla scoperta da parte di Luc Froment della situazione sociale esistente a Beauclair, costituisce per Zola l'occasione di mettere in scena una dura critica dell'industria capitalistica, quando si dimostra del tutto insensibile ai bisogni operai. La fabbrica è descritta come un mostro incombente sulla città, meritevole dell'appellativo con cui la indicano gli operai: l'Abîme, l'Abisso. L'ambiente dei proprietari, e quello ad essi alleato dei notabili e dei responsabili dell'amministrazione cittadina, è chiuso in una difesa a oltranza delle idee di proprietà e di eredità, e sente come una spinta all'eversione ogni richiesta operaia di miglioramento, anche moderato, delle condizioni del lavoro e dei salari. Il delitto più grave in una tale situazione, agli occhi di Zola, consiste proprio nell'offesa arrecata al lavoro: a Beauclair si respira "tutta la corruzione e tutta la degradazione del lavoro iniquo di molti per il godimento egoista di pochi, il lavoro disonorato, esecrato, maledetto, la spaventosa miseria che ne deriva, il furto e la prostituzione che ne sono come le ramificazioni mostruose" 168 .

Di fronte a una situazione del genere gli operai non hanno ancora elaborato un progetto efficace di raggiungimento della giustizia sociale. Alcuni si lasciano andare ad atti dimostrativi, distruttivi e violenti, che appena trascorsi lasciano le cose come prima; altri hanno puntato su una lotta collettiva come quella posta in essere dallo sciopero, ma senza avere idee precise su come costruire un mondo produttivo alternativo a quello esistente. A un certo punto Luc Froment incontra il capo dell'agitazione appena conclusa, un operaio dalle solide doti morali ma prigioniero della speranza nel comunismo di stato, sentito come l'unica possibilità di vedere il lavoro remunerato in modo equilibrato. Agli occhi di Zola gli operai non sanno bene con quali mezzi possano arrivare a un regime collettivistico, e comunque non sanno dove potrebbero collocare la libertà, in un sistema che sarebbe in ogni caso governato da "una macchina complicata di direzione e di controllo, che necessiterebbe di una polizia statale vessatoria e dura"169.

Esclusi così sia il modello capitalistico basato sul libero profitto, perché iniquo, sia il comunismo di stato, perché chimerico e illiberale, Zola si applica in Travail, per mezzo di Luc Froment, nella ricerca di un riscatto per il lavoro estraneo agli eccessi autoritari prevedibili se si affidassero tutti i mezzi di produzione allo stato. Questa ricerca è anzitutto una questione di pensiero, perché "il pensiero è un atto, il più fecondo che possa influire sul mondo"170. È in secondo luogo, e

\footnotetext{
${ }^{168}$ E. Zola, Travail, cit., p. 30.

${ }^{169}$ Ivi, p. 70.

${ }^{170}$ Ivi, p. 144.
} 
positivisticamente, un problema di analisi sociale, che sappia ridare coesione e giustizia a un mondo attraversato da una lotta di classe apparentemente insanabile. Ma è soprattutto un tema di riscoperta delle fonti teoriche della giustizia sociale del secolo XIX, da riproporre, come vedremo, alla luce delle nuove potenzialità offerte dal progresso scientifico. Ospite dei Jordan, Luc ha occasione di intrattenersi a lungo nella loro biblioteca, e qui trova le fonti di ispirazione che cercava, e cioè tutti "gli apostoli del Nuovo Evangelo: Fourier, Saint-Simon, Auguste Comte, Proudhon, Cabet, Pierre Leroux, altri ancora ..."171. In un periodo in cui si è ormai imposta una lettura in chiave conservatrice dell'opera di Comte, Zola continua invece a inserirlo all'interno di un elenco che comprende il meglio del pensiero socialista francese dell'Ottocento. Certo il nostro autore sa, e lo esprime attraverso le riflessioni di Froment, che si tratta di un elenco di personaggi dalle idee non proprio sovrapponibili. Ma se essi si presentavano divisi su talune soluzioni specifiche, erano per Zola comunque tutti uniti a formare un'avanguardia del tempo futuro. E quando lo scrittore vuole indicare che cosa al fondo li univa, lo fa in termini che impiegano volutamente più di un'intonazione comtiana. "La grande aurora che si levava dalle loro opere era la religione dell'umanità di cui tutti avevano avuto la fede, la loro compassione per i diseredati di questo mondo, il loro odio per l'ingiustizia sociale, la loro credenza nel lavoro salvifico" ${ }^{\text {"172 }}$.

Per la verità, più che Comte, il grande ispiratore del progetto zoliano è Fourier, il cui sistema è conosciuto dal romanziere soprattutto attraverso la lettura di Solidarité di Renaud ${ }^{173}$. La riflessione di Luc Froment sulla possibile alternativa al disprezzo del lavoro praticato dal capitalismo porta infatti in superficie molte idee proprie del fourierismo: la rivalutazione delle passioni (in esplicita polemica contro la mortificazione predicata dalla morale cattolica); la dottrina dell'armonia e la riorganizzazione sociale sulla base delle serie produttive; la speranza di cambiare il mondo gradualmente, partendo dall'esempio associativo offerto da comunità di moderate dimensioni; la concezione della società ideale come incontro fra capitale, lavoro e talento. All'impianto fourierista Zola aggiunge tuttavia un insistito richiamo all'uso sociale del progresso tecnico e scientifico, tanto da dichiarare la ragione umana infallibile, se mossa da giuste valutazioni etiche e rischiarata dalla

\footnotetext{
${ }^{171}$ Ivi, pp. 170-171.

${ }^{172}$ Ivi, p. 171.

${ }^{173}$ Solidarité, vue synthétique de la doctrine de Charles Fourier, è opera di Hippolyte Renaud uscita la prima volta a Parigi nel 1842, presso la Librairie de l'École Sociétaire, e più volte ristampata nel corso dell'Ottocento: ne esiste per esempio una sesta edizione del 1877. Ufficiale di artiglieria, convinto alle idee falansteriane da Victor Considérant, Renaud è, con lo stesso Considérant, uno dei più efficaci divulgatori del pensiero di Fourier. Zola riconosce il debito contratto con la sua opera mettendo in scena Luc Froment che, nella biblioteca dei Jordan, trova e legge appunto Solidarité. Sui rapporti fra il romanziere e la tradizione fourierista rinvio a M.A. Sarti, Fourier e Zola, Torino, Giappichelli, 1989. Altri contributi sul socialismo di Zola sono quelli di G. Genuzio, Jules Guesde et Émile Zola, ou le socialisme dans l'oeuvre de Zola, Bari, Levante, 1996, e di A. Vial, Germinal et le socialisme de Zola, Paris, Éditions Sociales, 1975.
} 
scienza. Il tutto è diretto, ancora una volta, all'emancipazione del lavoro, metafora dell'intero universo: emancipazione che deve passare attraverso la trasformazione del lavoro da salariato ad associato ${ }^{174}$.

La seconda parte di Travail, dopo quella di critica al sistema capitalistico privato, rappresenta la costruzione di un piccolo mondo industriale basato sui valori della cooperazione, in cui Zola si lascia affascinare da una vera e propria immagine utopica. Froment e Jordan decidono di recuperare a scopi produttivi una miniera abbandonata, vicino alla quale sorge presto una fabbrica in cui si realizza l'aspettativa dei due tecnici: porre la forza delle macchine al servizio del lavoro umano associato, al di fuori di ogni tentazione di sfruttamento. Dopo aver narrato le varie fasi, anche controverse (a causa della concorrenza e delle maldicenze poste in essere dalla borghesia del luogo), della formazione e dello sviluppo del nuovo progetto produttivo, Zola illustra alla fine la vita sociale che ne deriva, idealizzata appunto alla moda della letteratura utopistica. A un certo punto, per l'esplodere delle gelosie all'interno della famiglia che ne è proprietaria, l'Abîme, la fabbrica "cattiva", salta in aria. A Beauclair rimane solo la fabbrica "buona" dei Jordan e di Froment, che può condurre fino alle sue più luminose conseguenze l'esperimento di un'organizzazione equa del lavoro e di tutta l'esistenza sociale. $\mathrm{Ne}$ sono capisaldi l'integrazione fra produzione e consumo, la riduzione della durata e del peso del lavoro, assicurato comunque a tutti, il pensionamento generalizzato alla fine dell'età lavorativa, il raggiungimento della pace sociale e privata, la titolarità cooperativa della proprietà dei mezzi di produzione. Permangono differenze di classe, a favore dei direttori dell'azienda: ma i lavoratori possono finalmente affermare di non essere sfruttati, e di poter usufruire di tutti i vantaggi che una società progredita mette loro a disposizione.

Se il fourierismo ha offerto il quadro ideologico entro cui calare la comunità ideale immaginata da Zola, anche la cultura del positivismo ha contribuito in modo decisivo al successo dell'impresa, assicurando un progresso scientifico utilizzato interamente in innovazioni tecnologiche a vantaggio degli associati. A Beauclair si gira in specie di automobili elettriche, mentre nel mondo circostante vanno estendosi le comunicazioni aeree: comunicazioni più facili significano tra l'altro la possibilità di costruire una patria comune fra tutti gli uomini. Ovunque l'energia elettrica è protagonista di progressi consistenti, con la sua capacità di offrire luce, calore, movimento. Nella fabbrica di Froment e Jordan si lavora poco (la durata della giornata lavorativa è scesa progressivamente fino alle quattro ore) e con poca fatica, per merito dei macchinari messi in azione dai nostri inventori. Jordan sarà sorpreso dalla morte per vecchiaia mentre sta ancora studiando come "catturare" la luce solare del giorno per utilizzarla di notte, come fabbricare cioè un "sole

\footnotetext{
${ }^{174}$ L'entusiasmo di Froment rispetto a un programma del genere è evidenziato da questa riflessione: "il lavoro rimesso in onore, divenuto la funzione pubblica, l'orgoglio, la salute, la gaiezza, la legge stessa della vita. Sarebbe sufficiente riorganizzare il lavoro per riorganizzare l'intera società, di cui il lavoro dovrebbe essere l'obbligazione civica, la regola vitale" (É. Zola, Travail, cit., p. 173).
} 
elettrico" da porre sopra la città per illuminarla. Ricostruendo il cammino della fabbrica ideale che ha contribuito a impiantare, Luc commenta che "la scienza resta la grande rivoluzionaria". E aggiunge: "Solo la scienza e la verità emanciperanno sempre più l'uomo, lo faranno padrone del suo destino, gli daranno la sovranità del mondo, riducendo le forze naturali al ruolo di docili servitrici"175. È tuttavia avvertenza degli organizzatori dell'utopia di Beauclair di non spingere la tecnica a un'intensità tale da soffocare le caratteristiche umane: l'ambiente, reso più vantaggioso per l'uomo, mantiene tuttavia spiccati elementi "naturalistici", in modo che gli abitanti si sentano parte di una comunità produttiva ma inserita nella natura (anche per mezzo degli ampi parchi e giardini di cui dispongono) e capace di sentire le macchine alleate, e non più le nemiche che erano nella fabbrica sottoposta agli appetiti capitalistici ${ }^{176}$.

L'uso del progresso scientifico a fini sociali non è peraltro qualcosa di automatico, ma discende da una cultura complessiva che permea di sé i comportamenti degli abitanti di Beauclair. Su questo aspetto gioca un ruolo essenziale la credenza comune, che gli attori dell'utopia zoliana chiamano comtianamente "religione dell'umanità". Diverso dalle religioni tradizionali perché del tutto privo di dogmi, tale credo è incentrato sul principio dell'amore, che segue nella società ideale un processo di espansione molto simile a quello ipotizzato da Comte nel Système de politique positive. Il primo ambito di applicazione morale della legge dell'amore è la famiglia, nella quale si trovano le diverse tipologie parentali dell'affetto e della solidarietà. Già qui si manifesta uno dei caratteri fondamentali del sentimento che sostiene l'utopia zoliana, vale a dire l'amore verso la donna. Avviandosi alla vecchiaia, Froment ospiterà nella sua famiglia tre donne: la moglie; la sorella di Jordan, che diverrà di fatto una sorella anche per lui; e un'anziana signora rimasta sola, che sarà per Luc la raffigurazione dell'amore materno. Sembra quasi una rappresentazione di segno comtiano della funzione educatrice della donna, espressa attraverso una tipologia quasi completa dei sentimenti familiari visti dal lato femminile. Tutta questa rete di relazioni affettive trova poi il suo compimento nella cura della prole, come appare dalla preghiera, intonata alla religione dell'umanità, che Luc e la moglie recitano una sera: "Bisogna amare gli altri come amiamo noi stessi, è la stessa fiamma che avvicina tutti gli esseri, la nostra felicità di amanti e di sposi non può durare che nella felicità di tutti. Amore divino, poiché nulla può vivere senza di te, aiutaci a finire la

\footnotetext{
${ }^{175}$ Ivi, pp. 632-633.

176 "Sotto le immense vetrate trasparenti, si poteva credere di entrare in una città ben ordinata, gioiosa e ricca. Le macchine facevano ormai quasi tutto il bisogno. Azionate dall'elettricità, erano lì, superbe, a ranghi compatti, come un'armata di operai docili, infaticabili, pronti continuamente a offrire i loro sforzi. Se le loro braccia di metallo finivano per consumarsi, venivano semplicemente sostituite, ed esse ignoravano il dolore, avevano in parte eliminato il dolore umano. Era la macchina finalmente amica: non la macchina delle origini, una concorrente che aggravava la fame dell'operaio facendo abbassare i salari, ma la macchina liberatrice, divenuta lo strumento universale che penava al posto dell'uomo, affinché questi si riposasse" (ivi, p. 515).
} 
nostra opera, abbraccia i cuori, fa che tutte le coppie della Città amino e mettano al mondo figli, nell'universale predilezione che ci deve unire tutti" ${ }^{\prime 177}$.

Né l'amore, né la considerazione per la donna hanno tuttavia una funzione soltanto domestica, ma si allargano a comprendere sentimenti e pratiche di ordine sociale, soprattutto da quando il progresso scientifico ha condotto anche la donna a un livello di emancipazione uguale a quello dell'uomo. Un ruolo importante esercita in proposito, come è del resto nella tradizione utopica, l'educazione. A Beauclair esiste un vasto apparato scolastico, frequentato insieme dai giovani dei due sessi. Nelle fasce di età degli alunni più basse, l'istruzione è basata sulla conoscenza diretta delle cose e sull'acquisizione di un metodo cognitivo scaturente dall'osservazione della realtà, non dai libri: anche qui si sentono influssi fourieriani. A livelli più alti si affaccia un insegnamento scientifico più teorico, sempre intrecciato, tuttavia, con l'esaltazione della morale della solidarietà. Come in ogni utopia, i messaggi culturali ed educativi sono peraltro destinati a prolungarsi per tutta la vita dei singoli associati, che hanno a disposizione a tale scopo musei, biblioteche, teatri, feste.

L'asse ideale di ogni messaggio educativo resta ancorato alla mobilitazione morale, che dalla sfera privata deve prolungarsi alla sfera pubblica, fino alle sue più ampie dimensioni. Parlando ancora delle scuole, Zola afferma che gli studenti "crescevano insieme, vivevano sempre insieme. Solo l'amore era il legame di unione, di giustizia, di benessere. In esso si trovava il patto indispensabile e sufficiente, perché bastava amare affinché regnasse la pace. Questo amore universale, che si allargherà dalla famiglia alla nazione e dalla nazione all'umanità, sarà l'unica legge della felice Città futura" ${ }^{178}$. Con questo passaggio ci ricongiungiamo all'intenzione più autentica dell'utopia di Zola, che è la morale della solidarietà fra disuguali (perché talenti e competenze non sono gli stessi in tutti i soggetti) ma operanti per uno stesso principio etico, riassunto nella formula della religione dell'umanità.

\subsection{Anatole France e l'utopia come possibilità}

Fra gli scrittori attivi durante il passaggio fra Otto e Novecento, Anatole France è senz'altro colui che più riflette nelle sue opere la ricerca intorno al rapporto fra scienza e utopia. France è anche il letterato che più esprime tale problematica in termini dialettici, tanto che alcuni suoi scritti possono essere presi a sintesi dei temi che abbiamo finora incontrato: il confronto con la storia, le trasformazioni culturali indotte dalla civiltà industriale nel suo tecnicismo, la speranza o la paura nel futuro,

\footnotetext{
${ }^{177}$ Ivi, p. 473.

${ }^{178}$ Ivi, p. 228.
} 
l'approdo alla dimensione utopica come rappresentazione letteraria della possibilità o meno per l'uomo di dominare la propria evoluzione e quella del mondo ${ }^{179}$.

La formulazione più compiuta dell'incontro con la dimensione utopica di Anatole France è racchiusa nel romanzo Sur la pierre blanche ${ }^{180}$, pubblicato nel 1903. Si tratta di un'opera complessa ${ }^{181}$, sospesa tra passato, presente e futuro, in cui l'utopismo affiora almeno in due varianti: l'idealizzazione della cultura antica e la descrizione di una società immaginaria collocata nel futuro, che costituisce la vera e propria utopia racchiusa nella Pierre blanche. L'utopia del futuro, che riflette fra l'altro quel concorso fra scienza e ideologia che abbiamo visto essere anche in Zola uno dei caratteri fondamentali della speranza in un mondo più giusto, trova tuttavia collocazione e spiegazione all'interno dell'itinerario seguito da

\footnotetext{
${ }^{179}$ Vissuto fra il 1844 e il 1924, Anatole France (pseudonimo di François-Anatole Thibault), premio Nobel per la letteratura nel 1921, esordisce come poeta, ma incontra il primo successo di pubblico con il romanzo satirico Le crime de Sylvestre Bonnard, membre de l'Institut, del 1881. Nelle prove successive alterna il romanzo di ambientazione storica (come Thaïs e La rôtisserie de la reine Pédaque, entrambi dei primi anni novanta) con quello a sfondo sociale. All'inizio del Novecento, oltre alle opere a carattere utopico di cui si parla in questo testo, pubblica scritti come la dissacrante Vie de Jeanne d'Arc, del 1908, e il romanzo storico Les dieux ont soif, del 1912, un ritorno all'ambiente rivoluzionario del 1789 riletto alla luce dei valori di tolleranza. Per una visione complessiva dell'opera dello scrittore francese, che qui ci interessa solo per la discussione intorno all'utopismo, rinvio agli studi di N. Addamiano, Anatole France. L'uomo e l'opera, Padova, Cedam, 1947; A. Bruzzi, Aspetti, valori, fortuna di una critica: "La vie littéraire" di Anatole France, Bologna, Patron, 1959; C. Jefferson, A. France: the Politics of Skepticism, New York, Rutgers University Press, 1965; G. Todisco, Anatole France: littérature et engagement, Poggibonsi, Lalli, 1974.

${ }^{180}$ La spiegazione del titolo è offerta dal frammento posto in epigrafe: "Ti sembra di aver dormito sulla pietra bianca, in mezzo al popolo dei sogni". In più parti del libro viene in effetti posto in evidenza il tema del sogno come strumento di conoscenza e di progettazione, con un atteggiamento che chiama in causa, più che le nascenti teorie psicoanalitiche, proprio le tecniche di immaginazione tipiche della letteratura utopica. Anche nella conclusione ritorna esplicitamente l'argomento del sogno, le cui capacità evocative e creative sono comunque contrapposte alla difficoltà oggettiva di conoscere il futuro. Del resto tutto il libro ruota attorno al contrasto tra la fiducia di poter usare le operazioni della mente, comprese le loro forme meno scontate, per proiettarsi con una razionalità preveggente nell'esplorazione di un ipotetico futuro, e la limitatezza delle reali strategie di comprensione e di costruzione accessibili all'uomo.

${ }^{181}$ Sur la pierre blanche mette in scena un gruppo di intellettuali francesi di varia estrazione (un attaché d'ambasciata a riposo, un letterato, uno stampatore di forte cultura umanistica, un ingegnere, un critico d'arte), che vivono a Roma e sono soliti incontrarsi con Giacomo Boni, il noto archeologo italiano che nello stesso periodo in cui esce il romanzo sta lavorando al recupero del Foro. Ricordo che Giacomo Boni, vissuto fra il 1859 e il 1925, è tra i fondatori della scienza archeologica in Italia e tra gli iniziatori del metodo stratigrafico; nello stesso periodo in cui esce il romanzo di France, è protagonista di una serie di importanti scoperte riguardanti le epoche monarchica e repubblicana di Roma antica. Sur la pierre blanche propone una serie di dialoghi fra Boni e gli amici francesi, nei quali traspaiono da un lato l'intenzione di rappresentare Roma antica come città ideale, dall'altro le preoccupazioni riguardanti il presente e il futuro, che il gruppo di intellettuali vorrebbe più allineati a valori di esaltazione delle qualità umane. I discorsi fra i protagonisti di tali dialoghi sono alternati ai racconti che alcuni di essi narrano in occasioni conviviali, veri piccoli romanzi nel romanzo di cui i più significativi sono quello riguardante Gallione, e quello in cui viene ambientata l'utopia che conclude il libro: di ambedue questi episodi si parlerà diffusamente nel seguito.
} 
France nell'intera sua opera. Tale percorso va quindi seguito nei suoi elementi principali partendo dall'inizio, ossia dall' utopismo costruito attorno alla nozione di antichità, perché anche la celebrazione del passato serve a delineare le attese di un mondo ideale proiettato in avanti.

Il rimpianto della cultura antica espresso da France in quest'opera ruota attorno a due motivi strettamente connessi fra loro. Il primo è dovuto al riconoscimento della filosofia antica come modello di una conoscenza e di un'azione umane basate sulla centralità della ragione. France per la verità non si fa eccessive illusioni sulla capacità della ragione umana di dominare con sicurezza tutto il conoscibile: essa deve anzi scontare insufficienze ed errori che rendono più plausibili le posizioni venate di scetticismo rispetto a quelle che pretendono di costruire verità assolute. Eppure la ricerca filosofica, se ancorata alle dimostrazioni parziali offerte via via dalla scienza, rimane l'unico atteggiamento utile per dare consistenza all'irrefrenabile desiderio di sapere che muove gli uomini. Gallione, il protagonista di uno degli episodi centrali narrati nel libro, ambientato nel I secolo, esprime tale passaggio nell' argomentazione di France con queste parole, rivolte alla tradizione della filosofia greca, poi estesa al mondo romano: "I filosofi non sono mai d'accordo fra loro sul principio del mondo e ci lasciano nell'incertezza se la terra sia stata prodotta dall'acqua, dall'aria o, come è più credibile, dal fuoco sottile. Ma i Greci vogliono sapere tutto e fabbricano menzogne ingegnose. Come è meglio riconoscere la nostra ignoranza! Il passato ci è nascosto come l'avvenire; noi viviamo fra due nuvole spesse, nella dimenticanza di ciò che è stato e l'incertezza di ciò che sarà. E tuttavia ci tormenta la curiosità di conoscere le cause delle cose e un' ardente inquietudine ci spinge a meditare sui destini dell' uomo e del mondo"182.

Il secondo motivo che sorregge la visione nostalgica che France sembra avere della cultura antica risiede nel suo presunto spirito di tolleranza, strettamente collegato alla vena scettica della più autentica ricerca filosofica. Un pensiero in continua evoluzione, argomenta ancora Gallione, non può che presentare al proprio interno contraddizioni e zone d'ombra: tutte manchevolezze che autorizzano però, per un altro verso, un forte richiamo al valore della libertà delle opinioni. Questo principio non è solo un dato riguardante l'attività del pensiero, ma deve essere un

\footnotetext{
182 A. France, Sur la pierre blanche, in Oeuvres complètes illustrées de Anatole France, Paris, Calmann-Lévy, 1949-1952, t. XIII, pp. 393-394. L'episodio di Gallione trae ispirazione dagli Atti degli Apostoli (XVIII, 12-17), dove si racconta che i giudei di Corinto conducono a forza San Paolo presso le autorità romane insediate nel luogo, incolpandolo di tentare di persuadere a un culto contrario alla legge. Proconsole dell'Acaia è all'epoca Lucio Giunio Gallione, fratello del filosofo Seneca, il quale rifiuta di applicare la legge romana a un fatto del genere, perché nel comportamento di Paolo non sono ravvisabili delitti, ma questioni di parole o di nomi interne al credo ebraico su cui la giustizia romana non ha nulla da dire. Nel racconto di France Gallione viene presentato come un uomo politico illuminato, motivato dal suo stesso scetticismo alla tolleranza, anche se egli è incapace di cogliere nell'incontro con Paolo l'indicazione che va cercando riguardo al modo in cui tramonterà la civiltà romana. Gallione erra anche in altre cose (per esempio giudica il giovane Nerone una specie di principe-filosofo), ma non si sbaglia nel prefigurare l'importanza che la civiltà latina ricoprirà come eredità lasciata allo svolgersi della civiltà moderna.
} 
costume esteso all'ordine politico e sociale. "Conviene fondare l'ordine pubblico sulla diversità delle opinioni e non cercare di stabilirlo sul consenso di tutti alla medesima credenza. Questo consenso unanime non si otterrebbe mai e, sforzandosi di raggiungerlo, si renderebbero gli uomini tanto stupidi quanto furiosi. Infatti la verità più eclatante non è che un vano rumore per gli uomini ai quali viene imposta" ${ }^{\text {183. }}$.

Il principio della libertà di opinione è la condizione per cui si possono sviluppare, fra i protagonisti dei dialoghi compresi in Sur la pierre blanche, considerazioni che intrecciano spunti filosofici di ordine quasi materialistico e una critica incessante delle religioni del passato e del presente, giudicate sul metro della tolleranza o meno che praticano nei confronti di chi professa idee diverse. Le religioni convenzionali vengono in questo quadro ricondotte al livello di costruzioni della mente umana, transitorie come ogni prodotto storico. A un certo punto Anatole France fa trasparire il concetto che anche gli dei sono soggetti a consunzione da parte del tempo: unica dea di ordine superiore, perché strettamente legata alla dimensione della natura, è la Necessità. In questa storicizzazione relativistica delle credenze l'autore della Pierre blanche dimostra simpatia per i culti dell'antico Occidente, in quanto più vicini all'esperienza e alla ragionevolezza che dovrebbero guidare gli uomini nella loro ricerca di orientarsi fra le cose. Rispetto alle religioni monoteiste, il vecchio politeismo sembra essere meno capace di assorbire autoritariamente ogni sentimento di fede in immagini costruite una volta per tutte ${ }^{184}$.

Il rimpianto per la civiltà classica si aggiunge, nella scala dei gradimenti storici di France, alla critica espressa contro l'ebraismo e poi contro il cristianesimo, incolpati, alla maniera di Renouvier e in parte di Renan, di essere i responsabili principali dell'intolleranza che ha caratterizzato larga parte della storia d'Europa. Anatole France porta così anche il suo contributo alla polemica antisemita che attraversa un po' tutta la cultura europea a cavallo fra Otto e Novecento, cercando peraltro di mantenere i suoi dissensi all'interno di una contrapposizione culturale e non di matrice razziale. La polemica contro il razzismo è anzi uno dei temi maggiormente insistiti nella prima parte del libro. Uno dei protagonisti dei dialoghi, un umanista, afferma per esempio che "distinguere in un popolo le razze che lo compongono spesso è difficile quanto seguire nel corso di un fiume i corsi d'acqua che vi si sono gettati". Gli uomini di colori diversi "non sono razze, ma varietà della medesima razza, della stessa specie, che formano tra loro unioni

\footnotetext{
${ }^{183}$ A. France, Sur la pierre blanche, cit., p. 407.

${ }^{184}$ In un passo del libro Giacomo Boni afferma che "i Latini erano ragionevoli perfino nella loro religione. Conobbero dei piccini e volgari, ma pieni di buon senso e talvolta magnanimi” (ivi, p. 365). E continua contrapponendo il loro pantheon, composto di militari, di magistrati e di matrone, alle diavolerie preferite dagli Etruschi o alle figure grottesche proprie del cristianesimo medievale, giudicate espressioni mostruose di ignoranza e di paura. Vale la pena di sottolineare il fatto che France pone al centro dei suoi dialoghi un archeologo, cioè uno scienziato, per quanto di una disciplina particolarmente dipendente da impostazioni di tipo umanistico.
} 
feconde e si mescolano incessantemente. [...] Gli uomini immaginano le razze secondo il proprio orgoglio, o l'odio, o l'avidità. Nel 1871 la Francia fu smembrata a causa dei diritti della razza germanica, ma non c'è una razza germanica. Gli antisemiti accendono contro la razza ebraica la collera dei popoli cristiani, ma non c'è una razza ebraica" ${ }^{\text {"185. }}$. Escluso che si possano legittimamente distinguere gli uomini fra loro in base a criteri biologici, restano tuttavia le differenze di ordine culturale, che spesso si formano e si trasmettono da una generazione all'altra su basi etniche. Ed è su questo piano, esclusivamente culturale, che Anatole France si sente autorizzato a costruire una rappresentazione della civiltà antica dell'Occidente come luogo del relativismo religioso e della tolleranza, contrapposto al mondo ebraico-cristiano, caratterizzato invece dalla pretesa di possedere la nozione dell'unico vero dio.

La discussione intorno al cristianesimo costituisce l'occasione non solo di ribadire la preferibilità filosofica degli atteggiamenti tolleranti, ma anche di valutare l'opportunità di usare la riflessione razionale come apparato di pensiero avente possibilità di previsione. Il successo del cristianesimo delle origini si deve infatti anche al fascino indotto dalla delineazione di una strada di salvezza per gli uomini, di una prospettiva per il futuro. Per questa ragione gli umanisti che discutono nel romanzo di Anatole France dei modi di allontanarsi dall'intolleranza religiosa, si pongono nello stesso tempo il problema se un'operazione del genere possa essere più agevole se si accompagna l'indagine filosofica con ragionevoli previsioni riguardanti il futuro. Come sappiamo, si tratta di uno dei nodi centrali della cultura del positivismo, qui confermato dalla fiducia espressa da uno dei partecipanti ai dialoghi della Pierre blanche intorno alla capacità della scienza di costruire riferimenti praticabili per la conoscenza non solo di quel che è, ma anche del mondo che sarà: "La nostra conoscenza di ciò che sarà è in ragione della nostra conoscenza di ciò che è e di ciò che è stato. Più una scienza è esatta, più se ne possono trarre esatte profezie"186.

Sul piano generale dei comportamenti umani, le previsioni sul futuro trovano alimento dall'indagine scientifica della natura umana combinata con la conoscenza della storia. Anatole France crede in una certa dose di evoluzione "necessaria" riguardante l'umanità, che può essere esemplificata facendo riferimento a diversi ordini di fenomeni e alle loro condizioni storiche di realizzazione e di perfezionamento. Sul piano economico, la storia dei mezzi di produzione induce per esempio a pensare che il futuro metterà a disposizione dell'umanità una serie sempre più vasta di risorse, la cui distribuzione potrebbe richiedere (per necessità, non per giustizia) la sperimentazione su larga scala di metodi collettivistici. La conoscenza della storia delle religioni può far ipotizzare che anche il cristianesimo sia destinato a tramontare, per far posto a credenze più adeguate allo spirito del

\footnotetext{
${ }^{185}$ Ivi, p. 374.

${ }^{186}$ Ivi, pp. 466-467.
} 
progresso. Un'altra previsione, cui France tiene in modo particolare, riguarda la graduale costruzione della pace universale, frutto della crescita delle comunicazioni e della solidarietà fra gli uomini. La previsione si avvale di un atteggiamento più deterministico che fideistico: "La pace universale si realizzerà un giorno, non perché gli uomini diverranno migliori (non è concesso sperarlo), ma perché un nuovo ordine di cose, una nuova scienza, nuove necessità economiche imporranno loro lo stato di pace, come altre volte le condizioni stesse della loro esistenza li ponevano e li mantenevano in uno stato di guerra" ${ }^{\prime 187}$.

Fin qui l'argomentazione di France sembra allinearsi alla tentazione (presente anche in più di una frangia del positivismo) di descrivere un futuro più progredito come effetto di spinte interne al quadro dell'evoluzione naturale. Lo stesso perfezionamento morale, come appare dai riferimenti a una distribuzione equa delle risorse o all'avvento della pace universale, sembra più il prodotto di prevedibili condizioni oggettive che della mobilitazione soggettiva degli uomini e delle loro doti morali. Il discorso dell'autore della Pierre blanche intorno al progresso è però più articolato e meno deterministico di quanto appaia a un primo avvicinamento. Convinto, per effetto di una fiducia ricavata dalle scienze del suo tempo, della forza evolutiva insita nella natura, e in particolare nella natura umana, l'umanista France è tuttavia persuaso che il ruolo degli uomini dotati di ragione non sia soltanto quello di aspettare un progresso che comunque si avvererà, bensì quello di mettere le qualità dell'intelletto al servizio di una previsione cosciente dei fenomeni per guidarli. Il futuro insomma non va subìto, nemmeno nelle linee che il calcolo delle probabilità ci indica come evolutive, ma va capito preventivamente per essere dominato, modellato secondo le aspettative e le esigenze non solo materiali, ma anche morali, degli uomini stessi. Ritornando a Gallione, che si interroga incessantemente sui destini di Roma, France commenta che "è una disposizione ordinaria in spiriti di questo valore il cercare nel presente e nel passato le condizioni dell'avvenire. Io ho notato negli uomini più sapienti e meglio avvertiti che abbia conosciuto (Renan, Berthelot) una marcata tendenza a gettare, nel mezzo della conversazione, utopie razionali e profezie scientifiche" ${ }^{\text {"188 }}$.

${ }^{187}$ Ivi, p. 484. I partecipanti ai dialoghi alternano continuamente richiami all'antichità con riflessioni sul presente. Il discorso sulla pace universale costituisce per esempio l'occasione di una lunga digressione sulla guerra russo-giapponese che si sta sviluppando nello stesso periodo in cui è ambientato il racconto. Il quale si conclude con un fervente discorso antimperialista da parte di uno degli interlocutori: "Il grande valore umano è l'uomo stesso. Per mettere in valore il globo terrestre, occorre prima valorizzare l'uomo. Per sfruttare il suolo, le miniere, le acque, tutte le sostanze e tutte le energie del pianeta, occorre l'uomo, ogni uomo, l'umanità, tutta l'umanità. Lo sfruttamento del globo terrestre esige il lavoro combinato degli uomini bianchi, gialli, neri. Riducendo, diminuendo, indebolendo, in una parola colonizzando una parte dell'umanità, noi agiamo contro noi stessi. Il nostro vantaggio è che i gialli e i neri siano potenti, liberi e ricchi. La nostra prosperità e la nostra ricchezza dipendono dalla loro prosperità e ricchezza. Più produrranno, più consumeranno. Più profitteranno di noi, più noi profitteremo di loro. Lasciamo che godano in abbondanza del nostro lavoro, e noi godremo abbondantemente del loro" (ivi, p. 500).

${ }^{188}$ Ivi, p. 448 . 
L'utopia, intesa come articolata descrizione di società immaginarie, non è quindi fuorviante per lo spirito umano, ma costituisce anzi lo strumento con cui gli uomini si possono aprire al futuro come possibilità ${ }^{189}$. Certo, nell'epoca contemporanea non si può ormai che trattare di utopie aperte, perché la storia ci insegna che nulla può presentarsi come immutabile e che ogni situazione, anche la più progredita, può essere perfettibile e può in ogni caso prestare il fianco all'usura del tempo. Ma se le conoscenze e gli stessi comportamenti sono in divenire, non possiamo chiudere il campo delle possibilità a un realismo riduttivo, che sarebbe sterile per lo stesso ulteriore avanzamento della storia. Il futuro, insomma, non è campo d'esercitazione dei soli scienziati, ma anche degli utopisti: e France avanza in proposito, in termini di giudizio positivo per la loro opera, i nomi di Mercier, di Morris ${ }^{190}$ e di Wells, accanto a quelli già ricordati di Renan e di Berthelot (il Renan che più suggestiona France è naturalmente quello dell'Avenir de la science).

${ }^{189}$ Una tesi del genere era già stata espressa da Anatole France, e anzi con maggior estensione, nel Jardin d'Épicure, pubblicato nel 1895. In tale opera lo scrittore francese cercava di fare i conti con l'evoluzionismo darwiniano, che trasportato sul piano sociale significa una concezione evolutiva fatta di cambiamenti lenti, sottratti al controllo degli individui e anche delle singole comunità, in quanto proiettati su un movimento riguardante l'intera umanità e determinato da pulsioni in gran parte biologiche più che culturali. La saggezza umana dovrebbe però non accontentarsi di verificare il progresso, ma intervenire fin dove è possibile per determinare talune modalità del progresso stesso, per costruire per gli uomini un campo di scelta fra varie possibilità "secondarie" (data per scontata l'impossibilità di fermare il progresso). Il ruolo fecondo dell'utopia moderna risiederebbe secondo France proprio in questa indicazione di possibilità diverse dall'esistente. Con tale indicazione oltre tutto si mantengono in vita quelle qualità di immaginazione proprie della mente umana che il semplice adattamento alla realtà, anche se progressiva, farebbe lentamente scomparire. Al progresso determinato da forze oggettive si contrappone, commenta la Rota Ghibaudi, "una concezione di mutamento finalizzato, e quindi controllabile, non soltanto dalla ragione, ma anche dai bisogni, dai desideri, dalle aspirazioni, dalle speranze" (L'utopia e l'utopismo, cit., p. 43).

${ }^{190}$ News from Nowhere di William Morris, uscito nel 1890, costituisce uno dei modelli più seguiti nella scrittura di utopie a cavallo fra Otto e Novecento. Il romanzo dello scrittore e disegnatore inglese condivide peraltro solo per una parte gli umori più diffusi nelle utopie socialisteggianti del suo tempo. Un'analogia di tipo letterario risiede nel fatto che anche le Notizie da nessun luogo, come altri romanzi utopici che abbiamo fin qui incontrato, prende a pretesto la narrazione di un sogno per descrivere una società ideale posta nel futuro (in questo caso siamo nel 2003). Altre continuità con il modo in cui le utopie di fine Ottocento rileggono la tradizione del socialismo utopistico si ritrovano nella rappresentazione di un mondo retto dalla proprietà comune dei beni e organizzato secondo una federazione mondiale, che garantisce pace universale e decentramento amministrativo ed economico. Per altri versi, la società ideale di Morris non è però fortemente tecnicizzata, pur facendo buon uso delle conoscenze scientifiche e delle capacità industriali, e nemmeno proiettata verso una continua espansione economica. Più che tecnologico, il mondo ideale di Morris è estetizzante e naturalistico, guidato da una classe operaia in grado di determinare e accettare stasi produttive, se queste hanno la funzione di arrestare la competizione economica, di eliminare i consumi inutili e di perfezionare la solidarietà. Anche il lavoro, centro di ogni utopia, si uniforma a questi criteri, superando la distinzione fra lavoro intellettuale e lavoro manuale in una serie di occupazioni atte a rinsaldare $\mathrm{i}$ legami fra produzione, arte e costume sociale. Come al solito, non è possibile approfondire in questa sede i vari elementi delle utopie originate dalla cultura anglosassone, che qui si ricordano soltanto per gli indispensabili rinvii ai collegamenti esistenti fra le diverse culture nazionali che partecipano del mondo sviluppato di fine Ottocento e primo Novecento. La letteratura critica su Morris è tuttavia molto ampia, e ad essa può attingere il lettore per un'ulteriore documentazione. Ricordo in proposito i 
Si spiega anche attraverso questi passaggi il perché il nostro autore senta l'esigenza di concludere Sur la pierre blanche con una lunga digriessione dichiaratamente utopica, nella quale presenta un'umanità ideale organizzata, in modi non molto dissimili da quelli di Zola, secondo la confluenza di princìpi socialisteggianti e di atteggiamenti scientisti di origine positivista. Il narratore di questa utopia, Henry Dufresne, si trova proiettato per effetto di un sogno nell'anno 220 della federazione dei popoli, corrispondente all'anno 2270 dell'era cristiana. La nuova datazione ricorda il fenomeno decisivo che ha permesso la costruzione di una società ideale: il raggiungimento nel 2050 della pace universale, conclusione non solo dei conflitti interni all'Europa, ma anche delle tensioni imperialistiche proiettate in tutto il mondo. Altri fattori di trasformazione, che vengono comunicati al sognatore dagli abitanti del futuro, sono stati il successo politico delle idee socialiste, interpretate come il logico sviluppo della democrazia teorizzata nei secoli precedenti, e il passaggio della ricchezza prodotta dal capitalismo nelle mani di un proletariato emancipato. Gli uomini del futuro si sono infatti accorti che l'anarchia concorrenziale predicata e praticata dal capitalismo, se aveva un senso evolutivo in un mondo ancora scarsamente progredito, non ne ha più per una società in cui una produzione abbondante è assicurata dalla scienza alleata con il lavoro. Tutti i parametri riguardanti la ricchezza ricevono inoltre una nuova luce dalla capacità dei governi di assicurare l'equilibrio fra popolazione e risorse, anche con politiche di controllo demografico. Per questa ragione in quasi tutti i grandi stati si è passati gradualmente a una gestione sociale dei beni, che ha comportato l'abolizione della proprietà privata dei mezzi di produzione (ma non di quella dei beni di uso e di consumo): solo in Russia (e la notazione risulta evidentemente curiosa, visto l'anno di prima pubblicazione del libro di France) il passaggio al socialismo ha richiesto il ricorso a un movimento rivoluzionario.

L'utopia narrata da Anatole France in Sur la pierre blanche attraverso il racconto di Dufresne, segue in gran parte i canoni consolidati del genere utopicoucronico, con una certa attenzione per i vantaggi prodotti da un impiego socializzato dei progressi della tecnica. Il nostro viaggiatore nel futuro può visitare la Parigi del 2270 guidato da un operaio di nome Michel, il quale lo conduce in giro a bordo del suo piccolo aereo personale. Lo stesso Michel abita in una casa modesta ma gradevole, nella quale si trova installato un impianto idroterapico: altra

contributi di P. Meier, La pensée utopique de William Morris, Paris, Editions Sociales, 1972; E.P. Thompson, William Morris: romantic to revolutionary, New York, Pantheon Books, 1976; V. Fortunati, Utopia e sogno in "News from Nowhere" di William Morris, in AA.VV., Forme dell'utopia, Milano, La Pietra, 1979; AA.VV., William Morris: "News from Nowhere" cent'anni dopo, Napoli, Guida, 1992. Aggiungo che, oltre a quella di Morris, un'altra utopia, uscita due anni prima, sospinge alla fine dell'Ottocento critici e scrittori a riconsiderare i modelli di ricostruzione socializzata del mondo. Si tratta di Freeland. A Social Anticipation, di cui è autore l'esponente della scuola viennese di economia Theodor Hertzka. Il mondo anticipato da Hertzka è organizzato attorno a una struttura economica di cooperazione fra comunità di lavoro, in cui l'uso produttivo della tecnologia è tuttavia molto più vasto rispetto a quanto auspicato da Morris. 
occasione per celebrare il progresso tecnico e scientifico, unitamente alle abitudini salutiste che il proletariato del futuro ha ormai stabilmente acquisito. Nella società visitata da Dufresne vige un modello di rotazione del lavoro: Michel fa per sei ore il fornaio, poi partecipa insieme con altri a un gruppo di elaborazione di dati statistici (operazione importante, visto che "la statistica è la scienza che ha sostituito la storia" ${ }^{191}$ ). Come si vede, a differenza che in altre utopie, in quella di France si continua a lavorare per un numero rilevante di ore al giorno: questo perché il suo autore è convinto della funzione essenziale rivestita dal lavoro nel formare i caratteri degli uomini. La centralità del lavoro sorregge la speranza di poter costruire in futuro un mondo di soli operai. L'importanza del lavoro è confermata dal fatto che nella Parigi del 2270, in ossequio a un' aspirazione diffusa fra i socialisti utopisti del primo Ottocento, il denaro è stato eliminato, e i beni si scambiano sulla base delle diverse quantità di lavoro richieste per la loro produzione.

In sintonia con la letteratura utopistica, abbondano anche nel sistema di France le comunicazioni culturali e le iniziative educative, che si avvalgono di teatri, biblioteche, scuole, istituzioni artistiche. Le arti in particolare svolgono un ruolo rilevante nell'ammaestramento e nel perfezionamento degli abitanti, dopo che hanno soppiantato discipline inutili come il diritto (perché si compiono pochissimi reati) e la teologia, frutto di un pensiero considerato ormai arcaico. Vige comunque una larga tolleranza religiosa: sappiamo dal nostro visitatore che nella Francia del futuro sono presenti "la religione dell'umanità, il positivismo, il cristianesimo e lo spiritismo" 192 . Il cristianesimo ha peraltro assunto una veste molto più aperta e ideologicamente più blanda che in passato: la sua variante meno dialettica, il cattolicesimo, è ridotta alle dimensioni di una setta con poche migliaia di aderenti, e il laico France si concede il vezzo di ipotizzare a Roma l'esistenza di un papa a mezzo tempo, che di mestiere fa il tintore. La caduta di influenza delle religioni organizzate ha avuto conseguenze anche nel campo del costume: nel mondo del futuro non esiste più la famiglia in senso tradizionale, sostituita da una libertà sessuale priva di egoistici sentimenti di possesso, e con comportamenti più vari rispetto alla rigida codificazione dei costumi precedenti. Il riconoscimento della libertà sessuale deriva dal principio, di cui gli abitanti del futuro sono convinti, che "bisogna subordinare la società alla natura"193, e non viceversa.

$\mathrm{Al}$ caso della libertà sessuale non corrisponde tuttavia una concezione altrettanto liberale dell'intera associazione del futuro. I pubblici poteri, che hanno in mano i singoli stati e la federazione fra stati che garantisce la pace universale, agiscono anche per mezzo di divieti, di cui quello dell'iniziativa economica privata è il più importante. E come non è esaltata oltre misura la libertà, non è enfatizzato

\footnotetext{
${ }^{191}$ A. France, Sur la pierre blanche, cit., p. 524.

${ }^{192}$ Ivi, p. 549.

${ }^{193}$ Ivi, p. 543.
} 
nemmeno il motivo dell'uguaglianza: intellettuali e scienziati, che costituiscono il nucleo dominante del futuro, vivono in condizioni di maggior agiatezza rispetto ai lavoratori manuali, pur garantendo a tutti condizioni dignitose di esistenza. Quando Dufresne, il viaggiatore nel futuro, richiama in una discussione con operai parigini la triade rivoluzionaria del 1789, i valori cioè di libertà, uguaglianza e fraternità, i suoi interlocutori gli rimproverano di usare categorie di pensiero superate. "La libertà non può esistere nella società, perché non è nella natura. Non c'è un animale libero. [...] L'idea di uguaglianza è ancora meno ragionevole, ed è spiacevole in quanto presuppone un falso ideale. Noi non dobbiamo ricercare se gli uomini sono uguali fra loro. Dobbiamo vegliare affinché ciascuno fornisca tutto quel che può dare e riceva tutto ciò di cui ha bisogno. Quanto alla fraternità, sappiamo anche troppo bene come i fratelli hanno trattato i fratelli. Noi non diciamo che gli uomini sono malvagi. Né diciamo che sono buoni. Sono quel che sono. Ma vivono in pace quando non hanno motivi per battersi. Abbiamo una sola parola per esprimere il nostro ordine sociale. Noi diciamo che siamo in armonia" $" 194$.

La condensazione del ragionamento di France intorno alla parola chiave della scuola fourierista, appunto "armonia", ci riconduce alle ragioni per le quali il nostro autore tende a circondare la propria utopia di cautele rispetto all'ipotesi di un' organizzazione radicalmente compiuta di società ideale, costruita su impulsi morali superiori. Contano in ciò le propensioni dello stesso autore, che vuole disegnare un'associazione con una forte mobilitazione collettiva interna, ma non ugualitaria fino al punto di cancellare i meriti relativi di individui e categorie. In secondo luogo, France continua a presentare la società del futuro come il prodotto di spinte "naturali", conoscibili attraverso procedimenti scientifici, per quanto governate dalle capacità raziocinanti delle classi più consapevoli (appunto gli intellettuali e gli scienziati). La volontà di non costruire un'immagine "chiusa" della Parigi del 2270 deriva poi da un ulteriore atteggiamento mentale, quello legato alla necessità di coordinare le aspettative utopiche con i limiti alle possibilità previsionali posti comunque dalla scienza. Più felici degli uomini del tempo di France, gli abitanti del futuro non sono però pienamente felici, e sanno che ogni soddisfazione richiede sforzo, non è concessa con facilità né dalla natura né dalla storia. Non perfetta, la loro società è perfettibile, la qual cosa richiede di attingere a quel campo di conoscenze scientifiche e di illuminazioni utopiche cui si sono riferiti i parigini del XXIII secolo e i loro diretti progenitori per costruire un mondo migliore del precedente. Esiste insomma nell'utopia di France una sottile tensione tra la probabilità del progresso sociale e la limitatezza inevitabile di tale miglioramento, soggetto all'altrettanto limitata capacità del genere umano di cambiare in quanto specie. Tanto che il romanzo-apologo si conclude con una considerazione vagamente inquietante, anch'essa estrapolabile dall'osservazione scientifica. Se l'uomo non è stato il principio della terra, non è detto che ne debba

\footnotetext{
${ }^{194}$ Ivi, pp. 541-542.
} 
essere per forza la fine: altre specie più progredite potrebbero raccogliere il testimone dell'evoluzione...

\subsection{Il peso della storia}

Con il moltiplicarsi degli apporti intorno al tema delle relazioni fra scienza, utopia e morale, ci stiamo accorgendo come la riflessione avviata dalla cultura francese a cavallo fra Otto e Novecento sia complessa e non priva di elementi contraddittori. La conferma proviene dal fatto che l'oscillazione fra utopia positiva e visione catastrofica dell'avvenire finisce per attraversare con le sue incertezze l'interno della produzione degli stessi singoli autori. È quanto accade proprio ad Anatole France, che dopo aver auspicato al termine della Pierre blanche 1'avvento di un socialismo umanitario, nel giro di pochi anni si lascia prendere dalla tentazione di descrivere al contrario il futuro in termini distopici. Nel 1908, dopo averne anticipato il contenuto negli anni precedenti con alcuni estratti sul "New York Herald", France pubblica L'île des Pingouins, che si conclude appunto con una visione del futuro improntata a pessimismo. Il senso del libro dello scrittore francese non si esaurisce peraltro nella conclusione, ma risiede in tutto ciò che prepara un epilogo poco fiducioso sulle possibilità degli uomini di migliorare, se non se stessi, almeno il mondo che li ospita. Questa preparazione è la storia: che France, nella prefazione a L'Île des Pingouins, dichiara essere troppo complicata per poter essere oggetto di scienza. È opinione dello scrittore francese che la storia non sia ricostruzione oggettiva, alla maniera dei positivisti, bensì racconto, che egli decide di condurre in forma di apologo, con qualche concessione al gusto per la deformazione paradossale e con molte intenzioni di satira nei confronti della realtà.

Il racconto prende infatti le mosse da una situazione grottesca. San Mäel, un monaco che per la vecchiaia sta perdendo la vista, scambia per uomini i pinguini che abitano su un'isola, e li battezza: atto particolarmente sacro, che nel linguaggio elevato delle religioni, che France riproduce con intendimenti satirici, significa "Adozione, Rinascita, Rigenerazione, Illuminazione"195. L'errore del santo monaco provoca in paradiso una discussione su cosa fare dei pinguini battezzati, che per effetto dei significati simbolici appena elencati non possono più essere lasciati al loro destino animalesco, finché Dio decide di trasformarli in uomini e di consentire a San Mäel di trasportare la loro isola presso le coste bretoni. Da quel momento nasce il popolo dei Pinguini, che partecipano della natura umana declinata tuttavia secondo le specificità che diverranno loro proprie in quanto popolo. I "Pinguini" sono insomma i francesi, di cui l'autore de L'île des Pingouins si incarica di scrivere la storia alla ricerca dei loro caratteri particolari, insieme con quelli che segnano le esperienze di tutta l'umanità. In modo analogo a quanto già ipotizzato, seppur con intenzioni diverse, da un grande storico di fine Ottocento come

${ }^{195}$ A. France, L'île des Pingouins, in Oeuvres complètes illustrées, cit., t. XVIII, p. 40. 
Hippolyte Taine ${ }^{196}$, France muove il suo racconto da considerazioni di ordine antropologico, interpretando la storia come luogo in grado di offrire qualche indizio sulle caratteristiche universali dell'uomo. A differenza di Taine, egli non ipotizza tuttavia la sostanziale sudditanza degli uomini alle loro passioni: in modi al contrario più vicini a Rousseau, France sembra ipotizzare un'origine "buona" dell'essere umano, poi rovinato da una cattiva socializzazione. Originariamente i Pinguini sono infatti felici: ma quando il diavolo li convince dell'opportunità di coprire i loro corpi nudi comincia il distacco dalla felicità naturale. Finisce infatti il tempo della spontaneità amorosa, mentre l'apparizione dei primi veli induce i singoli Pinguini a sentimenti di distinzione rispetto ai loro simili. E questo è solo l'inizio della separarazione individualistica, che trova ulteriore alimento nelle violenze che accompagnano l'appropriazione privata dei campi. Commenta sarcasticamente France che tutto ciò avviene "per spirito d'associazione e previsione dell'avvenire. Perché l'uomo è per essenza previdente e socievole. Questo è il suo carattere. Egli non concepisce se stesso senza una certa appropriazione delle cose" ${ }^{\text {"197 }}$.

Come in Rousseau, la recinzione dei campi segna il punto fondamentale del passaggio a una civiltà basata sul diritto proprietario. Per quanto i Pinguini si sforzino di dare parvenza di legge a questa trasformazione, resta a lungo evidente che la proprietà poggia sul diritto di conquista, a sua volta origine dell'ossequio che i dominati manifestano più o meno spontaneamente alle élites formatesi in base alla forza. In effetti, quando nella loro prima assemblea come cittadini i Pinguini decidono di stabilire un'equa distribuzione delle imposte per sopperire alle

\footnotetext{
${ }^{196}$ Ricordo che Taine, molto più di France, è stato dai critici messo a confronto con la cultura del positivismo, soprattutto per le categorie di ambiente, razza e momento poste alla base della comprensione delle attività di pensiero (estetiche e filosofiche) e dello stesso comportamento umano. Il richiamo alle determinazioni spaziali e temporali dell'operare umano va in effetti a sovrapporsi al relativismo proprio della sociologia di impronta positivista, che ricerca nella storia il telaio dei condizionamenti esercitati dalla natura, ma anche le trasformazioni indotte dal trascorrere del tempo, inteso come accumulo di esperienze. Taine è però estraneo a una delle lezioni che il positivismo cerca di trarre dalla propria sociologia, e cioè l'idea del perfezionamento graduale dell'umanità. Orientato a sentimenti conservatori, Taine non crede al progresso, bensì costruisce una propria indagine storiografica basata sulla permanenza di determinate caratteristiche dell'uomo, che per la sua tendenza a essere preda delle passioni vive nel costante bisogno di poteri superiori che ne controllino gli atti. In un passo dell'Ancien régime questo atteggiamento di Taine risalta con particolare nitidezza, quando egli discute della fiducia professata dagli illuministi sulla ragione umana e obietta che i philosophes, a proposito della ragione, si erano "ingannati, e sotto due aspetti. Non soltanto la ragione non è naturale all'uomo né universale nell'umanità, ma nella condotta dell'uomo e dell'umanità, la sua influenza è piccola". Sono infatti altri, a parere di Taine, i "padroni dell'uomo". Sono "il temperamento, il bisogno fisico, l'istinto animale, il pregiudizio ereditario, l'immaginazione; in generale, la passione dominante, più particolarmente l'interesse personale o l'interesse di famiglia, di casta, di partito" (H. Taine, Le origini della Francia contemporanea. L'antico regime, Torino, Utet, 1961, p. 429). Sullo storico francese rinvio a C. Mongardini, Storia e sociologia nell'opera di H. Taine, Milano, Giuffrè, 1965, e soprattutto all'illuminante analisi di R. Pozzi, Hippolyte Taine. Scienze umane e politica nell'Ottocento, Venezia, Marsilio, 1993.
}

${ }^{197}$ A. France, L'île des Pingouins, cit., p. 77. 
necessità comuni, uno dei membri più forti della comunità si autoproclama nobile e impone di essere dispensato dall'obbligo della contribuzione. La polemica contro la nobiltà prelude a una visione dell'antico regime che France propone senza alcuna concessione allo spirito nostalgico proprio della storiografia di origine romantica, e in parte di quella positivista di impronta comtiana. Il medioevo è un'epoca di disordine e di imbarbarimento, nel quale i Pinguini sono sottoposti all'invasione della popolazione barbarica dei "Marsouins" (i Marsuini, dal nome di un tipo di cetaceo), allusione ai popoli germanici. Una speranza contro la decadenza dei costumi sembra a un certo punto provenire dalla cultura umanistica, quando una specie di Erasmo dei Pinguini, illudendosi sulle loro capacità di riflessione e di autocontrollo raziocinante, introduce il libero esame. Ma "egli portò con sé la riforma religiosa; i cattolici massacrarono i riformati; i riformati massacrarono i cattolici: questi furono i primi progressi della libertà di pensiero" ${ }^{\text {"198 }}$.

L'operazione critica di France riguardo al passato diviene tuttavia particolarmente netta quando egli tratteggia la storia contemporanea, dalla Grande Rivoluzione fino ai suoi giorni. In analogia con Taine, egli si fa condurre da una critica antiborghese, volta però a diffondere uno spirito corrosivo nei confronti della "modernità" e dei suoi valori solo in parte orientato al rimpianto tradizionalista, che è invece il segno precipuo delle intenzioni dell'autore delle Origines de la France contemporaine ${ }^{199}$. Anatole France ritrova le radici della contemporaneità nella critica avanzata contro le credenze tradizionali da parte dei philosophes: da loro nasce lo spirito che conduce alla distruzione dell'antico regime. La rivoluzione viene tuttavia criticata dall'autore de L'Île des Pingouins, non tanto perché violenta o "astratta" alla moda dei controrivoluzionari, ma perché

\footnotetext{
${ }^{198}$ Ivi, p. 177.

${ }^{199}$ Anche nell'opera storiografica di Taine spira una certa aria di polemica antiborghese, nel senso di critica agli eccessi di affarismo e di individualismo, che possono minare alla base i sentimenti di coesione sociale propri delle comunità tradizionali. L'oggetto più insistito della polemica di Taine è tuttavia lo stato, in particolare nelle sue forme democratiche che, in nome dell'uguaglianza, inducono alla costruzione di un ordine artificioso appropriato più a una caserma che a un'associazione di spiriti liberi. Il rapporto di Taine con il liberalismo costituisce comunque uno dei nodi più controversi per i critici che si sono interessati al suo pensiero. Da un lato egli sembra prolungare una preoccupazione propria della tradizione liberale, quella del rispetto della sfera privata come limite alla stessa azione della politica statale, in quanto necessaria al riconoscimento di sé dell'individuo come persona. Per un altro verso, la polemica antidemocratica di Taine sembra avere come riferimento positivo non tanto l'autonomia individuale, quanto l'intangibilità delle vecchie forme di associazione a base territoriale 0 corporativa. Mi pare che l'interpretazione più plausibile di questo intreccio sia quella di V. Collina, che nel saggio Ai margini del liberalismo: Taine storico della Francia contemporanea (in Il popolo e le élites, cit.) giudica tutto sommato abbastanza deboli i segnali di liberalismo presenti nell'opera di Taine, a fronte di molto più corposi ed evidenziati sentimenti di continuità tradizionalista con i miti aristocratici e disugualitari ricavabili dalla Francia prerivoluzionaria o da modelli autoritari più prossimi, come il bonapartismo. Altri autori, e in particolare E. Gasparini (La pensée politique d'Hippolyte Taine: entre traditionalisme et libéralisme, Marseille, Presses Universitaires d'AixMarseille, 1993), collocano invece il pensiero di Taine all'incrocio di un disegno equilibrato di apporti liberali e conservatorismo.
} 
non ha condotto a quel rinnovamento sociale promesso all'inizio. La responsabilità di tale "tradimento" è dei contadini e dei borghesi, per i quali la rivoluzione ha consistito in niente più che nell'appropriazione dei beni prima appartenenti ai nobili e al clero. All'influenza dei proprietari si deve anche la svolta successiva, quando il potere, in una repubblica ritenuta poco sicura per le proprietà, viene consegnato a Trinco (evidente trasfigurazione di Napoleone), la cui gloria deriva dalla guerra. Dopo varie peripezie, l'isola dei Pinguini ritorna a un regime repubblicano, ma dietro questa formula si nasconde in realtà il dominio di una parte sola della società, quella più ricca: "la democrazia pinguina non si governava affatto da sé: obbediva a un'oligarchia finanziaria che costruiva l'opinione mediante i giornali e teneva nelle sue mani i deputati, i ministri e il presidente" ${ }^{200}$.

S'inizia a questo punto la requisitoria di Anatole France contro la degenerazione affaristica della Terza Repubblica, preludio alla simpatia con cui assisterà, durante e dopo la prima guerra mondiale, ai tentativi rivoluzionari del proletariato europeo. La constatazione realistica che "ogni regime crea scontenti" in un primo momento, non preoccupare più di tanto il nostro "storico", che all'inizio delle allusioni alla politica francese del suo tempo indica fra gli scontenti della repubblica i nobili, il che non è poi un gran male. Sostenitori della repubblica sono invece, fra gli altri, anche gli operai, ai quali il nuovo regime offre una speranza dopo il violento tracollo della Comune. Ma lo sviluppo successivo della storia politica (che France esprime con allusioni al caso Boulanger, ritratto mentre trama con i tedeschi "marsuini", e all'affare Dreyfus) e della storia sociale, con l'instaurarsi progressivo del dominio dell'alta borghesia, sostengono e giustificano la denuncia contro un'organizzazione sociale ingiusta. Ne L'île des Pingouins si sviluppa a questo proposito una visione caricaturale dell'affarismo della Terza Repubblica, che si conclude con la seguente invettiva: "la Pinguinia si gloriava della sua ricchezza. Le cose necessarie alla vita mancavano a coloro che le fabbricavano, mentre abbondavano in coloro che non le producevano. Si tratta, come diceva un membro dell'Institut, di ineluttabili fatalità economiche. Il grande

${ }^{200}$ A. France, L'île des Pingouins, cit., p. 190. L'atteggiamento critico di France contro un sistema repubblicano governato dagli interessi di una minoranza di magnati si prolunga nel gustoso episodio in cui evoca il programma di un certo professor Obnubile (sotto la cui veste si cela un rimando a Tocqueville), che per studiare una vera democrazia decide di recarsi a Gigantopolis, capitale della Nuova Atlantide. Lì scopre che è vero che esiste un'autentica democrazia interna, la quale si sorregge però su continue guerre a scopi affaristici portate all'esterno. La riflessione di Obnubile è in proposito amara: "Poiché la ricchezza e la civiltà comportano altrettante cause di guerra della povertà e della barbarie, visto che la follia e la cattiveria degli uomini sono inguaribili, resta da compiere una sola buona azione. Il saggio deve ammucchiare dinamite sufficiente a far saltare il pianeta. Quando questo rotolerà a pezzettini attraverso lo spazio, un miglioramento impercettibile sarà realizzato nell'universo e sarà data soddisfazione alla coscienza universale, che peraltro non esiste" (ivi, p. 194). La pulsione distruttiva insita nell'immagine dell'esplosione definitiva del mondo, che non appartiene solo a France all'inizio del Novecento (si pensi alla conclusione della Coscienza di Zeno di Italo Svevo), ritornerà, come vedremo, al termine del racconto riguardante la storia dei Pinguini.

${ }^{201}$ Ivi, p. 197. 
popolo pinguino non aveva più tradizioni, cultura intellettuale, arti. I progressi della civiltà si manifestavano attraverso un'industria micidiale, la speculazione infame, il lusso schifoso. La sua capitale, come tutte le grandi città di allora, rivestiva un carattere cosmopolita e finanziario: vi regnava una laidezza immensa e diffusa. Il paese godeva di una perfetta tranquillità. Era l'apogeo",202.

La polemica contro l'affarismo del suo tempo prelude nell'opera di France a una visione altrettanto negativa dell'avvenire ipotizzabile, che non è in questo caso tanto un mondo governato dalla tecnica, quanto una società determinata dall'influenza del capitalismo e del suo denaro. L'attenzione distopica che conclude L'île des Pinguoins si volge anzitutto alla dimensione che assumerà nel futuro la capitale dei Pinguini, trasparente rinvio alla Parigi di domani: una città con quindici milioni di abitanti, piena di palazzi di trenta e quaranta piani, con un enorme inquinamento provocato dalle fabbriche circostanti. "Era la città più industriale e più ricca del mondo. La sua organizzazione pareva perfetta; non esisteva alcunché delle vecchie forme aristocratiche o democratiche delle società; tutto era subordinato agli interessi dei trusts" ${ }^{203}$. La società del futuro è in mano a un'aristocrazia di asceti del denaro, che celebrano i beni finanziari non come strumenti, ma come fini. France costruisce con intenzioni satiriche l'episodio di un capitano d'industria il quale, minacciato pistola alla mano da un operaio, che reclama in tale modo estremo un'indennità per un incidente sul lavoro che i tribunali ingiustamente gli negano, si lascia uccidere pur di non creare il precedente di un capitalista che ceda forzatamente un centesimo.

Nella descrizione del mondo futuro operata da L'Île des Pingouins, sotto l'alta borghesia dell'industria e della finanza si collocano altre due classi: il ceto medio dei tecnici e degli impiegati, del tutto asservito ai trusts, e la classe operaia, che costituisce l'unico gruppo sociale dal quale ci si dovrebbe attendere un moto di ribellione. Ma gli operai del futuro hanno perso molta della loro combattività, e sono pervenuti a una condizione di apatia alla quale non è estranea la mobilitazione della cultura ufficiale attorno ai soli valori dell'arricchimento. Mentre uomini e donne delle famiglie potenti sono additati come modelli sociali (intuizione non banale, in France, di tendenze della comunicazione culturale che saranno effettive nelle società capitalistiche sviluppate), e gli unici a essere esaltati sono i valori legati all'arricchimento individuale, "la decadenza fisica e morale degli operai era

\footnotetext{
${ }^{202}$ Ivi, pp. 394-395.

${ }^{203}$ Ivi, p. 400. Due anni prima che France pubblicasse la sua pessimistica immagine di un futuro dominato dal profitto del grande capitale, una tesi simile era apparsa nel romanzo di Jack London The Iron Heel. Nel Tallone di ferro il governo degli interessi capitalistici richiedeva tuttavia un maggiore impiego della violenza di stato (fra cui il dispiegarsi di conflitti imperialistici su scala mondiale) per arrestare il dissenso delle forze socialiste di opposizione. In France il sopore della coscienza di classe negli operai (pure realizzato, come vedremo subito) sembra il frutto più dell'egemonia sociale $\mathrm{e}$ culturale della borghesia capitalistica che non di un'integrale e aperta politica repressiva da parte dello stato.
} 
profonda; essi realizzavano il tipo di povero stabilito dall' antropologia"204. La scienza non gioca questa volta alcun ruolo progressivo nel favorire l'evoluzione umana: essa costituisce anzi uno dei puntelli principali di un regime che può apparire come il più stabile di tutta la storia, perché fondato su quelli che sono ritenuti i sentimenti più forti nella natura umana, e cioè l'orgoglio e la cupidigia.

Eppure gli spiriti più avveduti intuiscono, dietro la facciata di un mondo che sembra inattaccabile, le prime, profonde crepe. Le crisi di sovrapproduzione si moltiplicano; l'inquinamento e le speculazioni dell'industria alimentare diffondono malattie di origine ambientale; fra i ricchi, annoiati da una conquista del successo che non richiede più gli sforzi e le applicazioni di un tempo, aumentano i fenomeni di alienazione mentale e i suicidi; una tecnologia esplosa con troppa intensità rispetto alle capacità di contenimento dell'ambiente e di controllo dell'uomo, provoca ripetuti disastri negli impianti industriali e nei trasporti. Di fronte a tutto ciò, si assiste anche a una reazione morale da parte di una minoranza "anarchica": piccoli gruppi di dissidenti, nati da quegli stessi ceti medi resi schiavi dall'organizzazione economica e tecnico-scientifica imperante, cominciano a coltivare la nostalgia per un passato in cui il popolo dei Pinguini era meno disciplinato ma più gioioso. A un certo punto, di fronte all'impossibilità del sistema futuro di tacitare per davvero la lotta di classe, scoppia il meccanismo delle azioni e delle reazioni che condurrà alla catastrofe. Gli anarchici combattono la loro battaglia contro il regime a colpi di attentati dinamitardi, ai quali le forze dell'ordine reagiscono con crescenti misure di polizia. Ciò non basta tuttavia a ristabilire la calma, anzi vanno intensificandosi i fenomeni di panico e si moltiplicano le azioni sovversive, alle quali presto si aggiungono strati del proletariato, intenzionati a reagire alla loro povertà mediante saccheggi generalizzati. La capitale dei Pinguini è soggetta a ricorrenti esplosioni e incendi, finché disordini di particolare intensità la conducono alla totale distruzione provocata dal fuoco. La perdita della capitale dei Pinguini interrompe il flusso di ricchezza e conduce alla rovina l'intero sistema: si apre in pratica un nuovo medioevo, una nuova fase di invasioni barbariche, fino a che si porranno le condizioni per una nuova ascesa verso la prosperità. E dopo? Per un'umanità che dimostra in permanenza di non saper imparare nulla dalla propria storia, il France de L'Île des Pinguoins non intravede grandi speranze: ci sarà un'altra capitale di quindici milioni di abitanti, e altri grattacieli ... L'apologo si conclude insomma con umori diametralmente opposti rispetto alla Pierre blanche. Lì l'umanità riusciva a governare con una certa ragionevolezza il proprio destino, pur senza indulgere a eccessive speranze sulla possibilità di un perfezionamento capace di giungere a uno stadio di assoluta e definitiva compiutezza. Qui prevale invece un'interpretazione circolare della storia, i cui cicli di progresso e di decadenza sono

\footnotetext{
${ }^{204}$ Ivi, p. 403.
} 
comunque determinati da appetiti e sentimenti che fanno parte inesorabile della condizione umana.

\subsection{Un catalogo per il Novecento}

Iniziata sotto il segno dell' ambivalenza dell'eredità comtiana, la nostra rassegna si avvia alla conclusione senza aver incontrato alcun movimento di pensiero che abbia promesso per davvero di saper sciogliere i nodi principali riguardanti la costruzione del futuro, in un mondo fortemente segnato dalla conoscenza scientifica e dal progresso tecnologico. Il passaggio rapido di Anatole France dalla dimensione utopica alla visione distopica può essere preso a emblema di un'oscillazione fra speranza e paura che, come abbiamo visto, appartiene un po' a tutta la cultura dei continuatori o dei compagni di strada del positivismo (salvo le poche eccezioni saldamente ancorate o al versante ottimista o a quello catastrofista della questione).

La complessità di atteggiamenti culturali che accompagna di necessità la riflessione intorno ai rapporti fra scienza, utopia e morale, è del resto confermata nello stesso periodo da colui che ho già avuto occasione di citare come un interlocutore privilegiato per il pensiero francese a sfondo utopico di fine Ottocento e inizio Novecento: Herbert George Wells. Dopo essersi concesso più di una visione negativa intorno a un futuro dominato da semplici pulsioni materialiste o da intelligenze scientifiche puramente strumentali, Wells pubblica nel 1905 A Modern Utopia. E in essa, più che pretendere di imporre una parola conclusiva sul nodo, già comtiano, delle articolazioni fra ordine e progresso in una prospettiva futura, si incarica di esprimere la serie di tensioni etiche e intellettuali destinate a caratterizzare l'esistenza e il pensiero dell'umanità moderna. La prima di queste tensioni riguarda la stessa plausibilità o meno della dimensione utopica. Wells riprende e giustifica la ricorrente tendenza a immaginare situazioni diverse da quelle esistenti, valutando l'utopia un bisogno insopprimibile per l'uomo di praticare una dialettica fra sogno e realtà, il che comporta una ricerca di vitalità dell'immaginazione anche in un mondo che sembra appiattirsi nelle regole inflessibili dell'oggettività scientifica. La necessità dell'utopismo non dispensa tuttavia, a detta dello scrittore inglese, dalla necessità di rispettare nelle rappresentazioni ideali le acquisizioni fondamentali della scienza moderna: in particolare, la consapevolezza della dinamicità delle esperienze del genere umano, anche nel momento in cui si disegnano possibilità diverse da quelle esistenti ${ }^{205}$. La

\footnotetext{
${ }^{205}$ L'utopia come possibilità è da Wells sottolineata con l'artificio della presentazione in A Modern Utopia del nostro pianeta e di un suo gemello, che presenta via via gli stessi problemi del primo ma soluzioni diverse. Il carattere fortemente distintivo della Terra utopica, rispetto alla Terra conosciuta, è la presenza di una forte organizzazione scientifica che agisce su scala planetaria. La versione organizzata dell'evoluzione, che differisce profondamente dall'imperfetta spontaneità delle nostre esperienze, richiede un potere altrettanto forte: è questa la funzione dei "Samurai", ristretto nucleo di
} 
seconda tensione che va ricordata, a proposito di A Modern Utopia, è quella, che ci ha del resto accompagnato per gran parte del nostro itinerario dentro e ai margini del positivismo, fra la consapevolezza dell'ineluttabilità del progresso e l'emergere di dubbi sulla capacità dell'uomo di governarlo e di dirigerlo senza esporre il proprio genere a tensioni eccessive. Più che Comte, Wells ha presente a questo proposito la lezione di Darwin. E il suo sogno di un evoluzionismo organizzato esprime la speranza non solo di limitare i costi esistenziali del progresso, ma di poterlo gestire senza che l'artificialità propria delle costruzioni sociali avanzate riduca in modo eccessivo la spontaneità individuale. Ma su questo A Modern Utopia, pur cercando di convincere i suoi lettori che il principio della libertà non può essere affrontato nei mondi progrediti secondo gli stessi parametri della tradizione, continua a dibattersi nella contraddizione fra l'augurio di un governo razionale del progresso aperto a tutti, e il potere di controllo e di distinzione rispetto ai sudditi che le innovazioni tecnologiche sono in grado di conferire a chi detiene l'autorità.

Quando i legami fra utopia e scienza in una società moderna vanno chiarendosi nei termini problematici e insieme aggiornati che il progresso scientifico impone, la cultura che li ha in qualche modo generati è tuttavia ormai al tramonto. Ai primi del Novecento il positivismo ha perso gran parte del potere di coinvolgimento

\footnotetext{
sapienti che, anche nei comportamenti, un po' riproduce la congregazione dei filosofi sognata da Comte, un po' realizza le aspirazioni insieme scientiste e metafisiche di Wells. Un aspetto interessante è dato dal fatto che i Samurai alternano le funzioni di governo con periodi di allontanamento dal mondo, in cui si immergono nella meditazione personale. In ossequio alle tendenze moderne dell'utopia, che difficilmente riuscirebbe a mantenere le aspirazioni statiche della tradizione a confronto con lo sviluppo tecnico e scientifico (che Wells non vuole negare, ma anzi condurre a utilità generale), l'ordine non è costruito una volta per tutte secondo categorie precostituite, ma richiede un continuo adattamento al progressivo perfezionamento del mondo. Il tentativo di uscire dalla fissità propria del genere utopico si prolunga nella presentazione degli uomini del pianeta utopico come in perenne movimento, data la caratteristica "cinetica" del mondo contemporaneo mosso dalla tecnologia. Wells ci ricorda tuttavia che si tratta pur sempre di una società sottoposta al rigido controllo del potere: i Samurai sono infatti in grado di ricostruire in ogni momento la posizione di ciascun cittadino, che si presenta, come al solito in Utopia, trasparente all'occhio del potere. Non per questo, secondo lo scrittore inglese, la società ideale deve per forza essere tacciata di illiberale: la concezione della libertà si è più semplicemente adattata alle esigenze moderne, nelle quali non conta tanto la conservazione di una sfera di liceità precedente l'ordine politico, quanto la corretta comprensione di una dialettica costruttiva fra comando e libertà. Da un divieto possono nascere condizioni di libertà più sviluppate delle precedenti. Il caso più intuitivamente comprensibile in proposito è dato dal divieto di uccidere, che amplia, e non riduce, la presenza della vita e delle sue libertà. Ma non sono estranee a questo ordine di idee anche le questioni riguardanti l'organizzazione economica: il socialismo non è da interpretare solo come un insieme di costrizioni riguardanti la proprietà, ma anche come uno strumento per allargare tutta una serie di libertà che sono puramente formali finché gli uomini non sono sollevati dal bisogno materiale. Il fabiano Wells sottolinea peraltro che uno degli aspetti che meno condivide dell'utopismo del passato (che definisce predarwiniano) è l'imposizione dell'uniformità fra tutti i membri della società ideale. In A Modern Utopia è preoccupazione delle stesse autorità planetarie di consentire una ricerca e un riconoscimento autonomo di relazioni fra piccoli insiemi di cittadini che si associano spontaneamente, come avviene nella scelta e nella formazione dei gruppi di amici.
} 
intellettuale e di mobilitazione etica che lo contraddistinguevano mezzo secolo prima. Nella seconda metà dell'Ottocento la sua influenza, se non lo aveva mai portato a raggiungere quell'egemonia sulle classi subalterne sognata da Comte, lo aveva comunque posto fra i fondamenti culturali di una borghesia speranzosa di un progresso generalizzato, non troppo condizionato da egoismi di classe. All'inizio del nuovo secolo le borghesie dei paesi avanzati dell'Occidente invece si vanno sempre più rivolgendo a nuovi umori culturali, non tutti migliori del positivismo: si pensi, per fare un esempio, alla sostituzione della fede nella pace universale con nazionalismi di carattere aggressivo. Ma se il movimento fondato da Comte non ha saputo né costruire quella società positiva ideale che riteneva prossima, né risolvere i problemi individuali e collettivi della modernità, ha almeno contribuito a definire una delle parti più nuove del catalogo di problemi da affrontare con coscienza che l'Ottocento al crepuscolo consegna al nuovo secolo, e di qui ai nostri tempi. Su questo catalogo tornerò in conclusione. Prima può essere opportuno tentare di pervenire a un bilancio sul piano storiografico, e per stilarlo possiamo farci accompagnare dal protagonista delle ultime pagine di questo lavoro, Anatole France. Nell'estate del 1909 France compie un viaggio in Brasile, Uruguay e Argentina, tenendo una serie di conferenze che hanno spesso per argomento privilegiato il positivismo: una dottrina, introdotta in America latina soprattutto attraverso la mediazione della massoneria, che esercita una forte presa nelle borghesie di quei paesi, alla ricerca di una solida giustificazione del proprio ruolo di guida politica e sociale di fronte alle masse popolari. Di quelle conferenze, i cui testi sarebbero rimasti inediti fino a dopo la seconda guerra mondiale, le due per noi più significative sono dedicate l'una al padre fondatore del positivismo, Comte, l'altra al suo successore diretto, Laffitte, che era stato fra l'altro amico di France. L'interpretazione complessiva del movimento che lo scrittore francese ricava dalla propria testimonianza costituisce un'ulteriore spia del modo in cui un intellettuale di primo piano trasmette a inizio Novecento la tradizione positivista che gli sta immediatamente alle spalle.

Rispetto al positivismo, France dimostra un atteggiamento dialettico. Da un lato lo giudica la componente della filosofia occidentale dell'Ottocento che "ha creato senza dubbio qualcosa di più utile per lo spirito umano, di più conforme alle aspirazioni dello spirito moderno, almeno per l'epoca in cui questa dottrina fu concepita". Il positivismo è "il pensiero più nobile e imparziale di un'epoca di speranza e di inquietudine, il pensiero generoso che più potentemente di ogni altro (non dico vittoriosamente) ha tentato di conciliare la scienza e il sentimento, il cuore e lo spirito, la ragione e l'amore"206. L'altro lato dell'argomentazione di France, più critico, è dato dalla constatazione che il positivismo è progressivamente diventato una dottrina religiosa: e l'autore delle conferenze in Sudamerica, da buon

${ }^{206}$ A. France, Auguste Comte, in Oeuvres complètes illustrées, cit., t. XVII, p. 270. 
laico, sente il coinvolgimento in qualsiasi chiesa, vecchia o nuova che sia, come una limitazione per la libertà di coscienza.

Al di là di questa opinione generale, che France avanza in uno spirito di ammirazione verso Comte e di amicizia nei confronti di Laffitte, lo scrittore francese è interessato in particolare all'uso politico che si è fatto e si fa del positivismo: che è anche la ragione principale del nostro interesse intorno al movimento, e il primo dal quale ci siamo mossi per l'indagine racchiusa in questo libro. Anatole France giudica effimero e tutto sommato discutibile il successo politico ottenuto dal positivismo, soprattutto per merito di Littré, all'inizio della Terza Repubblica: discutibile perché l'adesione dei leader repubblicani alla dottrina positivista gli sembra superficiale e strumentale ${ }^{207}$. Di fronte all'ambiguità delle ricezioni ufficiali e di facciata dell'impianto positivista, di grande aiuto non è stato nemmeno il modo nascosto, ultraminoritario ed ecclesiale di interpretare il positivismo praticato da Laffitte, che ha avuto il torto di sprofondare nel silenzio anche quando occorreva parlare e prendere posizione, per esempio a proposito dell' affare Dreyfus. Per di più è spuntata (ed è il dato nuovo su cui ragiona a fondo Anatole France) una nuova professione di fede di origine positivista che, nel nome di Comte, cerca di trascinare l'idea della religione dell'umanità nel campo della reazione aperta. Lo scrittore francese, a proposito di questo ulteriore tentativo di appropriarsi della dottrina comtiana per egemonie politiche di corto respiro, cita le responsabilità generali della gioventù di destra del primo Novecento, e quelle particolari di taluni predicatori che a quella gioventù si rivolgono, come Maurras e Léon de Montesquiou. L'effetto di tutte queste intromissioni più o meno lecite nell'originario pensiero comtiano è che il positivismo, in Francia, è "tirato da due parti contemporaneamente, squartato fra i repubblicani e i monarchici. Mentre gli ortodossi, i successori regolari di Comte, lo trattengono nel campo repubblicano, nel libero pensiero, in una democrazia molto organizzata e ordinata ma alla fine progressiva e liberale, i teorici della monarchia integrale cercano di trascinarlo verso il diritto divino e la teocrazia" ${ }^{208}$.

France si chiede con insistenza se questo trascinamento sia fondato e, per una parte almeno, si dà una risposta affermativa. Incapace di considerare (come continua a fare invece Zola quasi negli stessi anni) Auguste Comte come una variante del pensiero socialista, Anatole France ne mette certo in luce gli elementi che non possono entrare a far parte del bagaglio intellettuale di un reazionario (l'idea di progresso, il sentimento repubblicano), ma ne individua anche, e forse

\footnotetext{
207 Dopo aver esplicitamente ricordato il pubblico apprezzamento per il positivismo espresso da personaggi come Gambetta e Ferry, France conclude: "I capi dell'opportunismo facevano appello, come viene viene, a una dottrina che raccomandava l'ordine come prima necessità sociale e politica (quell'ordine che questi antichi rivoluzionari saliti al potere avevano interesse a ristabilire sia nelle istituzioni sia nel costume) e si prefiggeva come scopo il progresso, termine che essi avevano molto utilizzato nella loro propaganda presso il popolo, senza annettervi un senso ben definito" (ivi, p. 274). ${ }^{208}$ Ivi, p. 278.
} 
con troppa insistenza, gli aspetti che possono suggestionare l'ambiente di formazione dell'Action française. Il primo elemento di analogia fra comtismo e reazione sarebbe, a giudizio di France, la comune polemica contro la rovinosità dei processi rivoluzionari, da sostituire con la riorganizzazione sociale. Nell'obiettivo di Comte, già disegnato nel 1822, di ricostruire la socialità mediante una "filosofia che metta termine all' anarchia mentale, e una morale e una religione che mettano termine all'anarchia morale" ${ }^{209}$, si nasconde magari insensibilmente un programma che, al di là della fraseologia sul progresso, può suggestionare più di un controrivoluzionario. Un secondo aspetto di convergenza, strettamente legato a questo, è dato dalla polemica antiparlamentare, solo in parte temperata dal riconoscimento del suffragio universale operato da qualche continuatore di Comte, ma non per ossequio al principio del numero. Ma il vero legame fra un Maurras e la tradizione comtiana risiede in considerazioni politiche ancora più profonde di quelle riguardanti specifiche istituzioni, e aventi a che fare con le grandi idealità spirituali. Sia i monarchici del 1909, sia Comte, sono a detta di France cattolici e religiosi. Per la verità Comte non può essere propriamente indicato come un cattolico: eppure quest'uomo "fortemente religioso" è anche "cattolico, perché nella sua fede è tradizionale e universale" ${ }^{, 10}$, ed è comunque responsabile di aver reso il positivismo un cattolicesimo senza Dio, che della vecchia religione ha trattenuto l'essenziale: sacerdozio, gerarchia, disciplina, sacramenti, cerimonie. Ultimo elemento di collaborazione potenziale fra positivismo comtiano e reazione è costituito dal principio autoritario. Al di là delle ambivalenze attraversate da Comte a proposito della libertà di pensiero, resta evidente che "lo spirito del dubbio non potrebbe accordarsi pienamente con lo spirito positivista" ${ }^{211}$. Ne è conferma il discutibile atteggiamento comtiano di ritenere la libertà di coscienza buona in tempi di lotta e infeconda in uno stato normale, tanto da desiderare di porre dei limiti allo spirito scientifico in nome dell'unità finale, fino ad asservire la stessa scienza alla religione. Il tutto è aggravato, a giudizio di France, dall' aspirazione del positivismo a trasferire i propri fondamenti nel campo della politica e della morale, dove la cancellazione della libertà di pensiero avrebbe conseguenze catastrofiche per la democrazia. Trascinare Comte nel campo della reazione non è insomma, secondo France, operazione che non trovi conferme negli scritti del fondatore del positivismo. Lo stesso conferenziere, seppur con meno insistenza, si dimostra tuttavia consapevole che l'iscrizione del positivismo comtiano nel campo della reazione suonerebbe abusivo nei confronti di molte altre parti, intellettualmente molto generose, dello stesso movimento culturale. Tali sono il desiderio di servire alla conoscenza attraverso una visione unitaria del mondo, l'autentico amore per

\footnotetext{
${ }^{209}$ Ivi, p. 280.

${ }^{210}$ Ivi, p. 283.

211 Ivi, p. 285.
} 
l'umanità, l'idea della pace universale, l'utopia della congiunzione fra organizzazione capitalistica della produzione e soddisfazione dei bisogni operai.

Nell'affresco dedicato in Sudamerica da France a Pierre Laffitte, a differenza di quello riguardante Comte sulle questioni culturali prevalgono i toni personali, derivati dalla frequentazione diretta ${ }^{212}$. Anche Laffitte, come Comte, ha avuto peraltro l'ambizione di trasportare il positivismo dal campo della conoscenza a quello delle applicazioni pratiche di ordine sociale e morale. Ma la ha espressa, secondo France, in modo meno autoritario: positivista integrale, ma non proselitista, Laffitte amava gli spiriti emancipati, cioè "indipendenti in materia religiosa, tolleranti, moderati",213. Per lo scrittore francese una notazione del genere non ha solo carattere biografico: sottolineare la possibilità di un altro atteggiamento positivista, diverso da quello comtiano, in merito all'indipendenza intellettuale, significa celebrare un positivismo alternativo rispetto a quello assunto superficialmente dai reazionari, e cioè il positivismo dell'indagine scientifica spregiudicata, della polemica anticristiana e anticattolica, delle società di libero pensiero.

Del modo di Laffitte di continuare la lezione del suo maestro Comte, Anatole France si sofferma su due aspetti, fra i più controversi della religione positiva, che qui tuttavia si colorano di nuove sfumature: il culto dei morti e il culto della donna. France racconta (e anche noi lo sappiamo, avendolo verificato con le sue memorie sui cimiteri ${ }^{214}$ ) che Laffitte era del tutto immerso nel culto dei morti: e aggiunge che i "suoi" morti, degni quindi di essere celebrati, erano tutti quelli ricordati dal calendario positivista. Per France un atteggiamento simile non è frutto di mera retorica o di esaltazione spiritualistica. Laffitte onora i morti per senso storico, perché le grandi personalità vivono di due esistenze: una che si conclude con la loro morte fisica, e una che si prolunga indefinitamente e dura finché si conservano la tradizione e il ricordo. Culto interamente terreno, nella ricostruzione effettuata da France, il ricordo dei "grandi" morti diventa espressione di un impegno vastissimo dell'individuo verso l'umanità. “Questa è la nobile perpetuità che il positivismo riconosceva all'anima umana, cioè all'insieme delle facoltà morali,

\footnotetext{
${ }^{212}$ La personalità di Laffitte, uomo semplice, vissuto e morto povero, come il suo maestro Comte, per l'analogo disinteresse verso i beni materiali, ma dotato di grande capacità di lavoro intellettuale, è tratteggiata da France facendo riferimento ai grandi odi che egli aveva manifestato durante tutta la sua vita. Il primo rifiuto sdegnato era contro Napoleone, per Laffitte un "uomo senza risultati"; il secondo era riservato a Victor Hugo, ritenuto dal seguace di Comte un retore romantico senza alcuna qualità intellettuale e morale, e nessuna autentica conoscenza storica. Il terzo odio di quest'uomo buono "si indirizzava, sempre ardente, contro un vecchio laborioso e disinteressato come lui, al quale la Francia e l'umanità dovevano grandi opere su soggetti diversi e importanti, e il migliore dei dizionari storici e critici della lingua francese: Littré. Perché Pierre Laffitte era così irritato contro questo sapiente dolce e buono, così laborioso e candido? Ah, perché? Domandate perché Calvino fece bruciare Michele Serveto! Littré era positivista, ma non era positivista allo stesso modo di Pierre Laffitte" (A. France, Pierre Laffitte, in Oeuvres complètes illustrées, cit., t. XVII, p. 308).

${ }^{213}$ Ivi, p. 318.

${ }^{214}$ Vedi, in questo libro, il par. 2.2.
} 
intellettuali e pratiche che caratterizzano l'uomo. Da ciò deriva fra l'altro che lo scopo della nostra vita, la sua regola e la sua destinazione, consistono anzitutto nel vivere oggettivamente per gli altri, al fine di vivere in seguito soggettivamente negli altri e per effetto degli altri; ovvero, in maniera più generale, consistono nel conoscere, amare, servire l'umanità",215.

$\mathrm{Al}$ di là delle indicazioni del calendario, Laffitte amava in particolare tra i suoi morti (continua Anatole France) Giulio Cesare e Augusto, perché fondatori dell'unico impero della ragione che sia esistito prima del secolo dei Lumi, e molte donne. Forse la ragione principale dell'apprezzamento per specifiche figure di donne (celebrate soprattutto perché compagne di grandi uomini) non è propriamente tale da soddisfare le tesi femministe, ma l'elenco prodotto da France sulla scorta dei ricordi riguardanti Laffitte è davvero di primo piano: Sophie Volland, la compagna di Diderot; madame Helvétius; Sophie de Grouchy, la moglie di Condorcet ("lo credo bene", commenta France); Clotilde de Vaux, la discussa ispiratrice delle esaltazioni amorose di Comte. Anatole France avrebbe visto bene, in simile compagnia, anche madame Roland: ma Laffitte non ne apprezzava il ricordo, perché era stata denigratrice e nemica di Danton ${ }^{216}$. Quanto alla de Vaux, France sembra incline a non calcare la mano sulle incontinenze immaginifiche di cui sarebbe stata responsabile presso Comte, il cui amore casto aveva finito per elaborare l'idea della partenogenesi della Vergine madre, "strana ispirazione di un amore ideale in un cervello in fiamme" ${ }^{, 17}$.

Critico severo delle infatuazioni del positivismo, specialmente nella sua versione comtiana, France è anche il personaggio che con serenità può tracciare la linea degli apporti che lo stesso movimento ha arrecato alla speranza dell'umanità di conoscere il mondo e di adattarlo alle sue esigenze universali. Lo scrittore francese si comporta tuttavia anche questa volta secondo il metro del suo abituale scetticismo, rifiutandosi di trarre una conclusione definitiva da quanto argomentato e narrato. Cosa può infatti derivare da tutte le osservazioni precedenti, favorevoli o contrarie che siano al progetto di rifondazione positivista dell'universo? Nulla. "Non bisogna mai avere fretta di trarre la conclusione dei fatti e delle idee. Sarà possibile farlo solo alla fine del mondo. E ci sono ancora possibilità che il mondo ricominci e che le conclusioni che avremo tracciato per questo siano false" ${ }^{218}$. Del

\footnotetext{
${ }^{215}$ Ivi, p. 321.

${ }^{216}$ Ricordo che, soprattutto negli anni intorno alla rivoluzione del 1848, i positivisti professano una specie di fede giacobina senza però avallare il mito di Robespierre. La loro idea di dittatura repubblicana trae ispirazione storica, più che dal Terrore, dal primo comitato di salute pubblica, quello diretto da Danton. Quella forma di concentrazione del potere sembra loro non solo meno sanguinaria, ma anche esercitata con il consenso delle organizzazioni popolari, e non espressione, come nel caso del robespierrismo, dell'aspirazione a una dittatura personale.

${ }^{217}$ A. France, Pierre Laffitte, cit., p. 323.

${ }^{218}$ Ivi, pp. 324-325.
} 
positivismo, si può ragionevolmente dire che "contiene delle verità, numerose verità, molte verità, ma non contiene la verità rigorosa, scientifica e totale" ${ }^{, 19}$.

E cosa possiamo ricavare noi, dal nostro viaggio intorno alle aspirazioni di condurre un mondo che progredisce scientificamente e tecnicamente a significati etici condivisi, dapprima nelle forme compatte della filosofia positiva e poi nelle più incerte espressioni della narrativa utopica? Si è già detto che, più che risposte, il dibattito su scienza, utopia e morale che si sviluppa in Francia (e altrove) nella seconda metà dell'Ottocento fornisce una nuova e argomentata serie di domande. Parlavamo in precedenza di un catalogo di problemi, che muove anzitutto dal rapporto tra scienza e credenza, nel contrasto fra l'estendersi, nella ricerca e nel costume, dell'abitudine a ricorrere all'oggettività del discorso scientifico, e il persistente bisogno di credenze forti, civili o religiose. Sul piano sociale, questa tematica si proietta come confronto-dissidio tra due culture: quella relativistica e strumentale della scienza e della tecnica, e quella che aspira a una concezione superiore di sé, in termini individuali o collettivi, ereditata dalla tradizione umanistica. Strettamente legato a questo problema è quello della collocazione dei valori umanistici in una società che si avvia sempre più verso un'evoluzione puramente economica e tecnologica. Il mondo costruito sulla base della scienza comincia già nell'Ottocento a somigliare a un ordine impersonale e oggettivo che l'uomo, almeno nella sua determinazione media, non sembra in grado di controllare. Non va inoltre dimenticato, come ulteriore elemento di discussione, che, agli occhi di molti osservatori critici delle modalità concrete della modernità, l'espansione tecnica e scientifica coincide, quasi di necessità, con un mondo a direzione capitalistica. E il capitalismo, nelle sue forme sviluppate, non significa più la concorrenza fra i meriti individuali di molti competitori, come sognava l'economia politica di fine Settecento, ma il monopolio del grande capitale e delle sue regole interne al profitto privato. Il tutto è infine segnato dall'ansia con cui si guarda al futuro e insieme al passato e al presente, con cui le speranze e i progetti si confrontano con la storia e con l'interpretazione deterministica che l'avvento delle scienze umane tende a conferirle. Più che per indicare soluzioni, a inizio Novecento c'è insomma materia per un elenco di nuove tensioni laceranti: fra immaginazione e realtà; fra desiderio di riconoscimento individuale e limitazioni proprie della specie e dell'organizzazione sociale; fra mito del progresso generalizzato e persistenza della disuguaglianza e del conflitto di classe; fra pratica strumentale indotta dalla tecnica e confusa aspirazione spiritualistica; fra tramonto inevitabile delle credenze tradizionali, soggette all'ulteriore processo della ragione, e resistenza a chiudersi nella semplice accettazione dell'esistente; fra coscienza dell'artificiosità storica delle esperienze umane, e nostalgia di una dimensione naturale a cui poter ancora attingere.

\footnotetext{
${ }^{219}$ Ivi, p. 325.
} 


\section{Indice dei nomi}

Abramo, 30.

Adam P., 85.

Addamiano N., 108.

Adriani M., 74.

Agostino (Sant'), 18.

Agrippa Menenio, 85.

Alessandro Magno, 68.

Annibale, 30.

Antoine E., 41.

Aquarone A., 37.

Archimede, 30.

Aristotele, 18, 29, 30.

Audiffrent G., 41, 44, 47.

Augusto: vedi Ottaviano Augusto.

Barral P., 40.

Barrès M., 98.

Becker B., 77.

Becker C., 101.

Beckett T., 30.

Beethoven L. van, 30.

Bellamy E., 100.

Bellini V., 30.

Bernard C., 17, 36, 37, 101.

Bernardo (San), 18.

Berthelot M.-P.-E., 36, 37, 47-56, 59, 86, 95,96 $112,113$.

Berthollet C.-L., 17.

Best J., 101.
Bichat M.-F.-X., 31.

Bisi R., 82.

Bizet G., 92.

Blanqui L.-A., 13.

Boisgilbert E., 84.

Bolívar S., 30.

Boni G., 108, 110.

Born F., 77.

Bossuet J.-B., 17, 18, 20, 30.

Boulanger J.-E., 90, 120.

Bowman S.E., 100.

Bradbury R., 93.

Bravo G.M., 13.

Broca P.-P., 36.

Brunetière F., 60, 61.

Bruno G., 68.

Bruzzi A., 108.

Bryant C.G.A., 35.

Buchez P.-J.-B., 12.

Budda, 30.

Buffon G.-L. Leclerc, 17.

Butler S., 80.

Cabanis P.-J., 18.

Cabet E., 104.

Calvino G., 128.

Carlomagno, 29, 30.

Carlo Martello, 30.

Cartesio: vedi Descartes R.

Casalini B., 57, 99.

Caselli, 78.

Cassina C., 12.

Cavallari G., 66.

Cellini B., 17, 30.

Cervantes M. de, 17.

Cesare Caio Giulio, 29, 30, 129.

Chardin, 17. 
Chateaubriand F.-R. de, 30.

Chesneaux J., 77.

Chiarini G., 101.

Cioran E., 94.

Cirio R., 74.

Collina V., 20, 66, 119.

Comenio, 30.

Commodo Marco Aurelio, 70.

Comte A., 5-33, 35-48, 52, 53, 60-61, 65-67, 75, 83 $85,91,93,98-100,102,104,106,124,125-129$.

Condorcet J.-A.-N. Caritat de, 11, 17, 18, 20, 26 40, 41, 53, 73, 129.

Confucio, 30.

Congreve R., 46.

Considérant V., 104.

Cook J., 17, 30.

Copernico N., 31.

Corneille P., 17.

Corra E., 47.

Costantino I, 70.

Cournot A., 37.

Dante Alighieri, 17, 29, 30.

Danton G.-J., 129.

Darwin C., 124.

Daudet L., 98.

Descartes R., 17, 29, 30.

Diderot D., 18, 129.

Diocleziano Gaio Aurelio, 70.

Donnelly I.: vedi Boisgilbert E.

Donzelli M., 84, 90.

Draper M., 76.

Dreyfus A., 120, 126.

Drumont E., 98.

Duby G., 35.

Duval M., 60.

Eliot G., 46.

Enfantin B.-P., 12.

Faguet E., 60-62.

Favari P., 74.
Federico II, 30.

Ferrarotti F., 82.

Ferry J.-F.-C., 40, 126.

Fimiani M., 84.

Fontenelle B. Le Bovier de, 17.

Fontpertuis H.-F. de, 37.

Fortunati V., 114.

Foucher L., 66.

Fourier C., 13, 18, 104.

France A., 55, 107-123, 125-129.

Francesco (San), 30.

Franklin B., 30.

Gallione Lucio Giunio, 108, 109, 112.

Gambetta L., 39, 40, 126.

Garibaldi G., 52.

Gasparini E., 119.

Genuzio G., 104.

Gesù Cristo, 30.

Gibbon E., 17.

Giovanna d'Arco, 30, 93.

Giovanni (San), 30.

Gobineau J.-A., 90.

Goncourt E.-H. e J.-H., 86.

Gondolo della Riva P., 77.

Gore K., 56.

Gourdin-Servenière G., 101.

Grange J., 12.

Grétry A.-E.-M., 17.

Grouchy S. de, 129.

Guicciardini F., 30.

Guizot F.-P.-G., 40.

Gutenberg J., 30.

Halévy D., 91-98.

Halévy E., 92.

Halévy L., 92.

Hamburger J., 37.

Hamelin O., 66.

Harp G.J., 99.

Harrison F., 41, 46.

Haussmann G.-E., 78. 
Hegel G.W.F., 13, 14, 30.

Helvétius Mme, 129.

Hérold F., 53.

Hertzka T., 114.

Holt L.E., 80.

Hudson R.H., 76.

Hugo V.-M., 81, 128.

Hume D., 7, 17, 18.

Huntington J., 76.

Huxley A.L., 93.

Ignazio di Loyola (Sant'), 30.

Jefferies R., 84.

Jefferson C., 108.

Jefferson T., 30.

Kagarlickij J.I., 76.

Kant I., 66-68.

Kolakowski L., 35.

Lafargue P., 36.

Laffitte P., 36, 37, 41-47, 49, 51, 67, 125, 126, 128 129.

La Fontaine J. de, 17.

Lamarck J.-B-P. de Monet, 17.

Lanaro S., 59.

Larizza M., 6, 41.

Lavielle V., 101.

Lavoisier A.-L., 17.

Le Bon G., 90, 91.

Legendre A.-M., 17.

Legrand L., 40.

Lenoir R., 78.

Leonardo da Vinci, 17.

Leroux P., 12, 104.

Licurgo, 30.

Littré E., 5-10, 21, 32, 36-42, 52, 68, 126, 128.

Lombroso C., 82, 85.

London J., 93, 121.

Luigi (San), 30.

Luigi XIV, 52.
Luigi XV, 52.

Luigi Filippo d'Orléans, 52.

Luigi Napoleone: vedi Napoleone III.

Macherey P., 12.

Machiavelli N., 31.

Macnie J.: vedi Thiusen I.

Magnin F., 41.

Maistre J. de, 18, 20.

Malagoli E., 100.

Malthus T.R., 89.

Mandeville B., 84.

Mandrou R., 35.

Mann E., 101.

Mantegazza P., 85.

Manuel F.E., 6, 26.

Manzoni A., 30.

Maometto, 30.

Marco Aurelio Antonino, 70.

Martineau H., 18, 46.

Massin C., 6.

Maurras C., 92, 98, 126, 127.

Meier P., 114.

Menichelli G.C., 101.

Mercier L.-S., 66, 84, 113.

Michelet J., 57.

Mignet F., 17.

Milet J., 82.

Milhaud G., 66.

Mill J.S., 46, 59, 96.

Molière J.-B. Poquelin, 17.

Mongardini C., 118.

Montesquieu C.-L. Secondat de, 11, 84.

Montesquiou L. de, 126.

Montgolfier J.-M. e J.-E., 30.

Monti A., 76.

Morgan A.E., 100.

Moro T., 93.

Morris W., 84, 113, 114.

Mosè, 30.

Mozart W.A., 30. 
Napoleone I, 120, 128.

Napoleone III, 6, 52, 57.

Negley G., 74.

Nerone Lucio Domizio, 109.

Nicolet C., 40.

Nietzsche F.W., 92, 97.

Omero, 17, 29.

Ottaviano Augusto, 129.

Orwell G., 93.

Pagès A., 101.

Paolo (San), 29, 30, 47, 109.

Paquot T., 102.

Patrick J.M., 74.

Péguy C., 92-93, 97.

Penn W., 30.

Petrarca F., 17.

Petrucci V., 84.

Pickering M., 12.

Platone, 18, 30.

Plutarco, 17, 30.

Poggi S., 35.

Pollini F., 77.

Polo M., 30.

Porta F., 76.

Pozzi R., 58, 118.

Prezzolini G., 93.

Proudhon P.-J., 13, 92, 95, 104.

Psichari H., 57.

Raby P., 80.

Renan J.-E., 37, 48, 50, 55-62, 110, 112-113.

Renaud H., 104.

Renouvier C.-B., 65-74, 84, 86, 110.

Rétat L., 56.

Rey A., 37.

Ribot T., 91.

Richelieu A.-J. du Plessis, 17.

Righetti L., 77.

Robertson W., 17.

Robespierre M.-F., 129.
Robin C., 36-38, 40.

Robinet J.-F.-E., 41, 46.

Roland J.-M. de la Platière, 129.

Romolo, 30.

Rossini G., 30.

Rota Ghibaudi S., 55, 74, 113.

Rousseau J.-J., 30, 54, 68, 118.

Roventi I., 80.

Saint-Simon H. de, 6, 12, 13, 18, 57, 104.

Saladino, 30.

Santucci A., 35.

Sarti M.A., 104.

Schopenhauer A., 13.

Scolari E., 101.

Scott J.A., 68.

Scott W., 17, 30.

Seneca Lucio Anneo, 109.

Serveto M., 128.

Shakespeare W., 17, 30, 58.

Simon W.M., 17, 35, 37, 41, 47, 48, 66.

Sorel G., 36, 92, 97.

Spencer H., 46, 99.

Stupuy H., 37.

Svevo I., 120.

Tacito Cornelio, 17.

Taine H.-A., 37, 61, 62, 118, 119.

Tarde J.-G., 82-91, 98.

Thibault F.-A.: vedi France A.

Thiers L.-A., 53.

Thiusen I., 100.

Thompson E.P., 114.

Tilgher A., 94.

Tocqueville A. de, 120.

Todisco G., 108.

Tommaso d'Aquino (San), 30.

Troyat H., 101.

Turgot A.-R.-J., 30.

Turris G. de, 93, 94.

Vaux C. de, 6, 19, 129. 
Indice dei nomi

Verne J., 74, 76-81.

Vial A., 104.

Virgilio Marone P., 17.

Volland S., 129.

Voltaire F.-Mn. Arouet, 17, 54, 84.

Ward L.F., 99.

Wardman W., 56.

Washington G., 30 .

Watt J., 30.
Webb B., 46.

Wells H.G., 65, 75, 76, 113, 123, 124.

Winckelmann J.J., 17.

Wright T.R., 46.

Wyrouboff G., 37-39.

Zola É., 37, 99-105, 107, 108, 114, 126.

Zoroastro, 30. 
Stampato da

Tipografia Editrice Polistampa

Via Livorno, 8

50142 Firenze 